\title{
Kombination von FADN- und IFCN-Datensätzen in der Politikfolgenanalyse - untersucht am Beispiel der EU-Milchmarktpolitik
}

\author{
Dissertation \\ zur Erlangung des Doktorgrades \\ der Fakultät für Agrarwissenschaften \\ der Georg-August-Universität Göttingen
}

vorgelegt von

Petra Thobe

geboren in Bonn 
1. Referent: Herr Professor Dr. Folkhard Isermeyer

2. Korreferent: Herr Professor Dr. Ludwig Theuvsen

Tag der mündlichen Prüfung: 06. November 2008 


\section{Inhaltsverzeichnis}

1 Einleitung 1

1.1 Problemstellung 1

1.2 Zielsetzung 2

1.3 Vorgehensweise 3

2 Struktur der europäischen Milchwirtschaft 5

3 Einzelbetriebliche Modelle in der quantitativen Politikfolgenabschätzung 16

3.1 Modelle in der quantitativen Politikfolgenabschätzung - ein Überblick 16

3.2 Entwicklungsstand modellgestützter Analysen in Deutschland und Europa 18

$\begin{array}{ll}\text { 3.2.1 Allgemeine Gleichgewichtsmodelle } & 23\end{array}$

$\begin{array}{ll}3.2 .2 & \text { Partielle Gleichgewichtsmodelle } \\ 3.23\end{array}$

3.2.3 Regionshof- und Betriebsgruppenmodelle 25

3.3 Anforderungen an einen geeigneten Analyseansatz 32

$3.4 \quad$ Schlussfolgerungen 38

$4 \quad$ Einzelbetriebliche Datengrundlage in der EU 39

4.1 Datengrundlagen in der quantitativen Politikfolgenabschätzung

- ein Überblick 39

4.2 Konzept der Europäischen Testbetriebsstatistik (FADN) 46

4.2.1 Zielsetzung 46

4.2.2 Entwicklung 47

4.2.3 Stichprobe landwirtschaftlicher Betriebe im EU-Testbetriebsnetz 49

4.3 Konzept des International Farm Comparison Network (IFCN) 62

4.3.1 Entwicklung und derzeitiger Stand 62

$\begin{array}{lll}\text { 4.3.2 Zielsetzung } & 64\end{array}$

4.3.3 Konzept typischer Betriebe 64

$\begin{array}{lll}4.4 & \text { Schlussfolgerungen } & 69\end{array}$

5 Ermittlung der Potenziale von FADN- und IFCN-Daten anhand von ausgewählten Analyseergebnissen $\quad 70$

5.1 Einsatz von FADN- und IFCN-Daten für Produktionskostenanalysen 70

$\begin{array}{lll}\text { 5.1.1 Produktionskostenanalyse mit FADN-Daten } & 70\end{array}$

$\begin{array}{lll}\text { 5.1.2 Produktionskostenanalyse mit IFCN-Daten } & 77\end{array}$ 
5.2 Einsatz von FADN- und IFCN-Daten für Politikfolgenabschätzungen auf dem EU-Milchmarkt

5.2.1 Ergebnisse bei Anwendung der FADN-Methode $\quad 79$

5.2.2 Ergebnisse bei Anwendung der IFCN-Methode 83

5.3 Schlussfolgerungen

6 Verknüpfungsansatz I: Einordnung und Auswahl der IFCN-Betriebe

6.1 Verknüpfungskonzept im Überblick 91

6.1.1 Konzept zur Einordnung der IFCN-Betriebe 92

6.2 Auswahl der FADN-Vergleichsgruppe 93

6.2.1 Selektion der Vergleichsgruppe mittels euklidischer Distanz 94

6.2.2 Statistische Einordnung typischer IFCN-Betriebe in die Verteilung der FADN-Betriebsgruppe $\quad 99$

6.2.2.1 Problemstellung bei der statistischen Einordnung 99

6.2.2.2 Herangehensweise anderer Wissenschaftler 100

6.2.2.3 Bewertung des Entwicklungsstandes 103

6.2.2.4 Eigene Lösungsansätze 103

6.3 Aufbereitung des FADN-Datenmaterials zur statistischen Einordnung der typischen Betriebe

6.3.1 Berechnung der FADN-Produktionskosten in Anlehnung an die IFCN-Methodik

6.3.2 Methodisches Vorgehen bei der Auswahl der Untersuchungsstichprobe

6.4 Analyse der Verknüpfung der FADN- und IFCN-Datensätze zur Einordnung der Betriebe

6.4.1 Wahl der Untersuchungsregionen und -betriebe

6.4.2 Ähnlichkeit der FADN-Vergleichsbetriebe und Wahl der FADN-Vergleichsgruppen

6.4.3 Bewertung der Einordnung von IFCN-Betrieben mittels FADN

6.4.4 Diskussion

6.5 Auswahl von typischen IFCN-Betrieben mit Hilfe der Clusteranalyse

7 Verknüpfungansatz II: Aufbereitung von FADN-Daten zur Nutzung im Modell TIPI-CAL

7.1 Verknüpfungskonzept im Überblick

7.1.1 Konzept zur Modifikation von FADN-Daten zur Simulation im Modell TIPI-CAL

7.2 Methodisches Vorgehen bei der Simulation von FADN-Betrieben

7.2.1 Projektion von FADN-Betrieben

7.2.2 Spezifikation des Modells zur Schätzung von Koeffizienten 
7.2.2.1 Entwicklung der Schätzung von Koeffizienten

7.2.2.2 Komponenten und Wirkungszusammenhänge bei der Koeffizientenschätzmethode

7.2.2.3 Berechnung und Minimierung der Korrektur-,,Kosten“ 150

7.2.2.4 Einschränkungen der Nutzbarkeit der Koeffizientenschätzmethode

7.3 Analyse der Verknüpfung von FADN- und IFCN-Daten bei der Simulation von Politikänderungen am Beispiel des Milchmarktes

7.3.1 Modifizierung des FADN-Datensatzes für Simulationsrechnungen im Modell TIPI-CAL

7.3.2 Bewertung der TIPI-CAL-Modellrechnungen mit FADN-Datensätzen

7.3.4 Diskussion und Schlussfolgerungen 


\section{Abbildungsverzeichnis}

Abbildung 2.1: $\quad$ Entwicklung der Betriebsstrukturen in der EU-15

Abbildung 4.1: $\quad$ Organisation des Informationsnetzes landwirtschaftlicher

Buchführungen

Abbildung 4.2: $\quad$ Klassifizierungsschema für die Berechnung der Hochrechnungsfaktoren

Abbildung 4.3: Datenerhebungsverfahren zur Erfassung typischer Betriebe

Abbildung 5.1: $\quad$ Milchpreise, Produktionskosten und Gewinnspannen in spezialisierten Milchviehbetrieben der EU-15, 2003, in c/kg

Abbildung 5.2: $\quad$ Kumulierte Verteilung der Milchviehbetriebe, sortiert nach der Höhe der Vollkosten und der Vollkosten abzüglich kalkulatorischer Größen (Beispiel: Frankreich)

Abbildung 5.3: Vollkosten der Milchproduktion abzüglich Nebenerlöse (Beispiel: Frankreich)

Abbildung 5.4: $\quad$ Produktionskosten in Abhängigkeit von der Betriebsgröße

Abbildung 5.5: $\quad$ Produktionskostenvergleich typischer IFCN-Betriebe für den Betriebszweig Milch (Teil 1)

Abbildung 5.5: $\quad$ Produktionskostenvergleich typischer IFCN-Betriebe für den Betriebszweig Milch (Teil 2)

Abbildung 5.6: $\quad$ Änderung der Milchproduktion, Referenz zu Basisjahr

Abbildung 5.7: $\quad$ Änderung der Milchproduktion bei 50 \%iger Kürzung der LFA+AUP, \% zu REF_SQ

Abbildung 5.8: $\quad$ Projektion der Entwicklung von typischen Milchviehbetrieben (IFCN) in Deutschland, Schweden und Spanien in der Baseline (1999 bis 2008)

Abbildung 5.9: $\quad$ Folgenabschätzung des MTR-Beschlusses und eines fiktiven Milchquotenausstiegs am Beispiel von drei deutschen IFCNBetrieben

Abbildung 6.1: $\quad$ Vergleich von IFCN-Betrieben mit FADN-Betrieben

Abbildung 6.2: $\quad$ Anteil der milchproduzierenden Betriebe an der monetär bewerteten landwirtschaftlichen Erzeugung (für 27 landwirtschaftliche Produktionsgebiete in 1999/2000)

Abbildung 6.3: $\quad$ Kumulierte euklidische Distanz in Abhängigkeit von der Anzahl abgebildeter Betriebe aus der Grundgesamtheit 
Abbildung 6.4: Kumulierte Euklidische Distanz in Abhängigkeit von der abgebildeten Milchmenge aus der Grundgesamtheit (in Tonnen)

Abbildung 6.5: $\quad$ Einordnung der IFCN-Betriebe in die FADN-Verteilung: auf Basis der Produktionskosten - Betrieb D-68 (SH-NI)

Abbildung 6.6: Einordnung der IFCN-Betriebe in die FADN-Verteilung auf Basis der Produktionskosten - Betrieb D-35 (BY)

Abbildung 6.7: Konstruktionsprinzip bei der hierarchischen Klassifikation

Abbildung 7.1: $\quad$ Schematische Darstellung zur Erweiterung des FADN-Datensatzes für Simulationsrechnungen im TIPI-CAL-Modell

Abbildung 7.2: $\quad$ Entwicklung des betrieblichen Gewinns (in Euro) in der Baseline mit und ohne betriebliche Anpassung beim FADN-Betrieb im Vergleich zum typischen IFCN-Betrieb

Abbildung 7.3: $\quad$ Entwicklung des Gewinns im Betriebszweig Milch Euro pro $100 \mathrm{~kg}$ Milch) in den Szenarien MTR-Beschluss und Milchquotenausstieg beim FADN-Betrieb im Vergleich zum IFCNBetrieb ohne Anpassung

Abbildung 7.4: $\quad$ Entwicklung des Gewinns im Betriebszweig Milch (Euro pro $100 \mathrm{~kg}$ Milch) nach betrieblicher Anpassung beim FADNBetrieb im Vergleich zum IFCN-Betrieb 


\section{Tabellenverzeichnis}

$\begin{array}{lll}\text { Tabelle 2.1: } & \text { Entwicklung der Kuhmilchproduktion in Europa } & 7\end{array}$

Tabelle 2.2: $\quad$ Kuhmilchbilanz der Europäischen Union 8

Tabelle 2.3: $\quad$ Übersicht über den Handel von Milcherzeugnissen (EU-15)

Tabelle 2.4: $\quad$ Entwicklung der Anzahl Milchkühe und der durchschnittlichen Herdengröße in der EU (2000 bis 2004) 10

Tabelle 2.5: $\quad$ Entwicklung der Anzahl an Betrieben mit Milchvieh $\quad 11$

Tabelle 2.6: $\quad$ Durchschnittliche Milchleistung je Kuh und Jahr $\quad 11$

Tabelle 3.1: $\quad$ Hauptgruppen von Produktions- und Betriebsmodellen für die Politikanalyse

Tabelle 3.2: $\quad$ Beschreibung von verschiedenen Forschungseinrichtungen eingesetzter Modellen zur Analyse der Wirkungen des MTR auf den EU Milchmarkt (Zeitraum 1999 bis 2002)

- Auszug aus der Anhangstabelle A3.1

Tabelle 3.3: $\quad$ Beschreibung der von verschiedenen Forschungseinrichtungen eingesetzten Modelle zur Analyse der Wirkungen des MTR auf den EU-Milchmarkt (Zeitraum 1999 bis 2002)

- Auszug aus der Anhangstabelle A3.2

Tabelle 3.4: $\quad$ Modellierung im EU-Milchsektor - Überblick (Produktions- und Betriebsmodelle grau markiert)

Tabelle 4.1: $\quad$ Überblick über Agrarstatistiken in Deutschland und in der EU

Tabelle 4.2: $\quad$ Schichtung der INLB-/FADN-Stichprobe

Tabelle 4.3: $\quad$ FADN-Stichprobe und Durchschnittsgewicht

(Rechnungsjahr 1997, LBS 1997, StDB 1994)

Tabelle 4.4: $\quad$ Erfassungsbereich des FADN (LBS 1997/Jahr der landwirtschaftlichen Betriebsstrukturerhebung)

Tabelle 4.5: $\quad$ Fehlerklassen

Tabelle 5.1: $\quad$ Änderung der Milchproduktion in Prozent bei Übergang von zwei Übertragungsregionen zu nationalem Quotenhandel

Tabelle 6.1: $\quad$ Median und Standardabweichung der FADN-Vergleichsgruppe zum typischen 68-Kuhbetrieb in Bremervörde/Niedersachsen bei unterschiedlicher Datenbasis

Tabelle 6.2: $\quad$ Verteilung der Milchkühe gemäß der Betriebstypen (\%) 
Tabelle 6.3: $\quad$ Durchschnittliche Bestandsgrößen in den europäischen Milchviehherden (gemessen in Rindergroßvieheinheiten)

Tabelle 6.4: $\quad$ Beschreibung der ausgewählten typischen Milchviehbetriebe

Tabelle 6.5:

Prozentualer Anteil der abgebildeten Milchviehbetriebe in Deutschland, Schweden und Spanien in Abhängigkeit von der Ähnlichkeitszahl

Tabelle 6.6: $\quad$ Prozentualer Anteil der abgebildeten Milchproduktion in Deutschland, Schweden und Spanien in Abhängigkeit von der Ähnlichkeitszahl

Tabelle 6.7: $\quad$ Prozentualer Anteil der abgebildeten Milchviehbetriebe aus der Grundgesamtheit

Tabelle 6.8: $\quad$ Mittelwert und Standardabweichung bei unterschiedlicher Repräsentativität der verschiedenen FADN-Vergleichsgruppen zum IFCN-68-Kuhbe-trieb in Niedersachsen

Tabelle 6.9: $\quad$ Mittelwert und Standardabweichung bei unterschiedlicher Repräsentativität der verschiedenen FADN-Vergleichsgruppen zum IFCN-35-Kuhbe-trieb in Bayern

Tabelle 6.10: $\quad$ Vergleich zwischen zwei ausgewählten deutschen IFCNBetrieben mit ihren FADN-Vergleichsbetrieben (Jahr 1999)

Tabelle 6.11: $\quad$ Ergebnisse der Selektion in der Region Nord-West-Deutschland

Tabelle 6.12: $\quad$ Ergebnisse der Selektion in der Region Mitte- und Süddeutschland

Tabelle 6.13: $\quad$ Ergebnisse der Selektion in der Region D-Ost (spez. Milchviehbetriebe)

Tabelle 6.14: $\quad$ Ergebnisse der Selektion in der Region D-Ost (alle Betriebe mit Milchvieh)

Tabelle 7.1: $\quad$ Ableitung der Inputvariablen zur Berechnung der Arbeitskosten

Tabelle 7.2: $\quad$ Annahmen für das Referenzszenario

Tabelle 7.3: $\quad$ Annahmen für den MTR-Beschluss (Juni 2003) und für das Szenario Milchquotenausstieg

Tabelle 7.4: $\quad$ Wichtige Betriebskennzahlen in der Ausgangssituation und nach einem Wachstumsschritt auf 120 Kühe 


\section{Kartenverzeichnis}

Karte 5.1: $\quad$ Vollkosten der Milchproduktion auf Basis von FADN-Daten in Euro/dt (Wirtschaftsjahr 1999/2000)

Karte 5.2: $\quad$ Differenz zwischen Milcherlösen und den Vollkosten bei einer Milchpreissenkung um 20 \%; Datenbasis: FADN-Daten (Wirtschaftsjahr 1999/2000)

Karte 6.1: $\quad$ IFCN-Netzwerk typischer Milchviehbetriebe in der EU

(Stand: Oktober 2002) 


\section{Verzeichnis der Abbildungen und Tabellen im Anhang}

\section{Anhang zu Kapitel 2}

Tabelle A2.1: Milchquoten: Gesamtreferenzmenge je Mitgliedstaat (1999/2000)

Tabelle A2.2: $\quad$ Angebot und Verbrauch von Käse in der EU-25

Tabelle A2.3: $\quad$ Angebot und Verbrauch von Butter in der EU-25

Tabelle A2.4: $\quad$ Angebot und Verbrauch von Vollmilchpulver in der EU-25

Tabelle A2.5: $\quad$ Angebot und Verbrauch von Magermilchpulver in der EU-25

\section{Anhang zu Kapitel 3}

Abbildung A3.1: $\quad$ Modelle im Modellverbund der Bundesforschungsanstalt für Landwirtschaft (FAL)

Abbildung A3.2: $\quad$ Aufbau des Betriebsgruppenmodells FARMIS

Tabelle A3.1: $\quad$ Beschreibung ausgewählter Modellansätze im Rahmen des MTR für den EU-Milchmarkt (1999 bis 2002) - Teil 1

Tabelle A3.1: $\quad$ Beschreibung ausgewählter Modellansätze im Rahmen des MTR für den EU-Milchmarkt (1999 bis 2002) - Teil 2

Tabelle A3.1: $\quad$ Beschreibung ausgewählter Modellansätze im Rahmen des MTR für den EU-Milchmarkt (1999 bis 2002) - Teil 3

Tabelle A3.2: $\quad$ Beschreibung der Szenarien und Ergebnisse ausgewählter Modellansätze im Rahmen des MTR für den EU-Milchmarkt (1999 bis 2002) - Teil 1

Tabelle A3.2: $\quad$ Beschreibung der Szenarien und Ergebnisse ausgewählter Modellansätze im Rahmen des MTR für den EU-Milchmarkt (1999 bis 2002) - Teil 2

Tabelle A3.2: $\quad$ Beschreibung der Szenarien und Ergebnisse ausgewählter Modellansätze im Rahmen des MTR für den EU-Milchmarkt (1999 bis 2002) - Teil 3

\section{Anhang zu Kapitel 4}

Tabelle A4.1: $\quad$ Festlegung unterschiedlicher Auswahlschwellen (in ESU) in den verschiedenen EU-Mitgliedsländern zur Auswahl der Betriebe für die FADN-Stichprobe (von der Kommission angewandt für das Jahr 1997) 


\section{Anhang zu Kapitel 6}

Tabelle A6.1: $\quad$ Eigenschaften der 17 bedeutendsten Milchviehbetriebstypen

Tabelle A6.2: $\quad$ Schlüssel für die Aufteilung der Kosten auf die Milcherzeugung

Tabelle A6.3: $\quad$ Schätzung und Aufteilungsschlüssel für die familieneigenen Produktionsfaktoren

Abbildung A6.1: Ablaufdiagramm zur Kontrolle der FADN-Arbeitsaufwendungen

Tabelle A6.4: $\quad$ Weitere Datenkontrollen

Tabelle A6.5: $\quad$ Strukturelle Entwicklung von Milchviehbetriebe in Deutschland

Tabelle A6.6: $\quad$ Strukturelle Entwicklung von Milchviehbetriebe in Schweden

Tabelle A6.7: $\quad$ Strukturelle Entwicklung von Milchviehbetriebe in Spanien

Tabelle A6.8: $\quad$ Ergebnisse der Selektion in der Region Nord-West-Deutschland (spezialisierte Milchviehbetriebe)

Tabelle A6.9: $\quad$ Ergebnisse der Selektion in der Region Mitte- und Süddeutschland (spezialisierte Milchviehbetriebe)

Tabelle A6.10: $\quad$ Ergebnisse der Selektion in der Region Ostdeutschland (spezialisierte Milchviehbetriebe)

Tabelle A6.11: $\quad$ Ergebnisse der Selektion von allen Betrieben mit Milchkühen aus dem FADN in der Region Ostdeutschland

\section{Anhang zu Kapitel 7}

Tabelle A7.1: $\quad$ Verschiedene Stufen der Auswertungen

Tabelle A7.2: $\quad$ Rechenverfahren zur Verknüpfung der FADN-Daten im TIPI-CAL Modell - Teil 1

Tabelle A7.2: $\quad$ Rechenverfahren zur Verknüpfung der FADN-Daten im TIPI-CAL Modell - Teil 2

Tabelle A7.2: $\quad$ Rechenverfahren zur Verknüpfung der FADN-Daten im TIPI-CAL Modell - Teil 3 


\section{Abkürzungsverzeichnis}

$\mathrm{AB}$

AFPC

AK

ASE

AWU

BAL

BEMO

BGB

BMELF

BML

BMVEL

BNP

BSE

BZA

CA

CAPMAT

CAPRI

CARD

CBS

CEEC

CEEC-ASIM

D

DBV

DEA

DEFRA

DFG

DRAM
Agrarberichterstattung

Agricultural and Food Policy Center der Texas A \& M University, USA

Arbeitskraft

Agrarstrukturerhebung

Annual Work Unit

Institut für Betriebswirtschaft, Agrarstruktur und ländliche Räume der Bundesforschungsanstalt für Landwirtschaft, Braunschweig

Einzelbetriebliches Optimierungsmodell basierend auf repräsentativen Betrieben

Bürgerliches Gesetzbuch

Bundesministerium für Ernährung, Landwirtschaft und Forsten

Bundesministerium für Ernährung, Landwirtschaft und Forsten

Bundesministerium für Verbraucherschutz, Ernährung und Landwirtschaft

Preisindex des BNP??? (Kap. 4, S. 106, Allok. Fam.)

Bovine Spongioforme Enziophalopathie

Betriebszweigabrechnung

Chambre d'Agriculture

Common Agricultural Modelling Accounting Tool

Common Agricultural Policy Reform Impact Analysis

Center for Agricultural and Rural Development, Iowa State University, Kansas City/USA

Central Office of Statistics

Central and Eastern European Countries

Central and Eastern European Countries Agricultural Simulation Model

Deutschland

Deutscher Bauernverband

Data Envelopment Analysis

Department for Environment, Food and Rural Affairs, London/UK

Deutsche Forschungsgesellschaft

Dutch Regional Agricultural Model 
dt

EDF

EG

EGE

EMZ

ESIM

ESVG

ETH

EU

EUROSTAT

EWG

FADN

FAL

FAO

FAPRI

FARMIS

FCM

FLIPSIM

FWU

GAP

GAPsi

GATT

GeWiSoLa

GD

GLS

GTAP

$\mathrm{GuV}$

HGB

I-/O-Koeffizienten

IAP

IFCN
Dezitonne

European Dairy Farmers

Europäische Gemeinschaft

Europäische Größeneinheit

Ertragsmesszahl

European Simulation Modelling Framework

Europäisches System Volkswirtschaftlicher Gesamtrechnung

Swiss Federal Institute of Technology, Zürich, Schweiz

Europäische Union

Statistisches Amt der Europäischen Gemeinschaften

Europäische Wirtschaftsgemeinschaft

Farm Accountancy Data Network

Bundesforschungsanstalt für Landwirtschaft Braunschweig

Food and Agriculture Organization of the United Nations

Food and Agricultural Policy Research Institute

Farm Group Model for the German Agricultural Sector

Fat Corrected Milk

Farm Level Income Policy Simulation Model

Family Work Units

Gemeinsame Agrarpolitik

Gemeinsame Agrarpolitik simuliert

General Agreement of Tariffs and Trade

Gesellschaft für Wirtschafts- und Sozialwissenschaften des Landbaus

Generaldirektion

Grazing livestock systems

General Trade Analysis Project

Gewinn-und-Verlust-Rechnung

Handelsgesetzbuch

Input-/Output-Koeffizienten

Institut für Agrarpolitik, Marktforschung und Wirtschaftssoziologie der Universität Bonn

International Farm Comparison Network 
INEA

INLB

INP

INRA

INRADM

KFZ-Steuer

KtBL

KULAP

LBS

LEI

LF

LGR

LP

LU

LwG

LZ

MA

MDM

MIPsi

MS

MTR

NBL

NUTS

OECD

PMP

RAUMIS

REGIO

RICA

RGV

SDB
Instituto Nazionale di Economia Agraria, Parma, Italien

Informationsnetz landwirtschaftlicher Buchführungen

Input Sheet (Eingabeseite im TIPI-CAL Modell)

Institute National de la Recherche Agronomique

INRA Dairy Model

Kraftfahrzeugsteuer

Kuratorium für Technik und Bauwesen in der Landwirtschaft e.V. Darmstadt, Deutschland.

Kulturlandschaftsprogramm

Landwirtschaftliche Betriebsstrukturerhebung

Agricultural Economic Research Centre, The Hague, Niederlande

Landwirtschaftliche Nutzfläche

Landwirtschaftliche Gesamtrechnung

Lineare Programmierung

Livestock Units

Landwirtschaftsgesetz

Landwirtschaftszählung

Institut für Marktanalyse und Agrarhandelspolitik der Bundesforschungsanstalt für Landwirtschaft Braunschweig

Manchester Dairy Model

Milchmarktpolitik, simuliert

Mitgliedstaat

Mid-term Review (Zwischenbewertung)

Neue Bundesländer

Nomenklatur von statistischen, territorialen Einheiten (siehe Kap. 3 unter REGIO)

Organisation for Economic Co-operation and Development

Positive Mathematical Programming

Regionalisiertes Agrar- und Umweltinformationssystem

Eurostat Datenbank für harmonisierte Regionalstatistiken

Französische Bezeichnung für das europäische Testbetriebsnetz

Rindergroßvieheinheit

Standarddeckungsbeitrag 
SLI

SPEL

StBA

StDB

StLÄ

TEAGASC

TIPI-CAL

UK

USA

VE

VO

VZ

WATSIM

WAU

WJ

WRC

WTO

XA-Solver

ZMP
Swedish Institute for Food and Agricultural Economics, Lund/ Schweden

Sektorales Produktions- und Einkommensmodell für die Landwirtschaft

Statistisches Bundesamt

Standarddeckungsbeitrag

Statistische Landesämter

Irish Agriculture and Food Development Authority

Technology Impact and Policy Impact Calculations

Vereinigtes Königreich

United States of America

Vieheinheit

Verordnung

Landwirtschaftliche Vergleichszahl

World Agricultural Trade Simulation Model

Wageningen University, Niederlande

Wirtschaftsjahr

„Weltregionenzentren“ (IFCN)

World Trade Organisation

Optimierer zur Lösung linearer und mixed-integer Probleme, es enthält einen veränderten primal simplex Algorithmus, einen dual simplex Algorithmus und einen „interior point barrier“ Algorithmus

Zentrale Markt- und Preisberichtstelle der Land-, Forst- und Ernährungswirtschaft $\mathrm{GmbH}$ 


\section{$1 \quad$ Einleitung}

\subsection{Problemstellung}

Der Mid-term Review ${ }^{1}$ (MTR)-Beschluss aus dem Jahr 2003, die EU-Osterweiterung mit zehn neuen Beitrittsländern und die laufenden WTO-Verhandlungen werden die Rahmenbedingungen der EU-Landwirtschaft weiter grundlegend bestimmen. Besonders betroffen von der fortschreitenden Liberalisierung der Agrarpolitik ist der stark regulierte EU-Markt für Milch und Milchprodukte.

Um die Verhandlungsposition zu verbessern und um rechtzeitig möglicherweise notwendige Begleitmaßnahmen zu entwickeln, ist es für die Politik wichtig, vorab über die Wirkungen der Reformen und der sich ändernden Rahmenbedingungen auf die EUAgrarproduktion informiert zu sein. Hier ist die agrarwissenschaftliche Politikberatung gefordert.

Der Beitrag der Wissenschaft zur Abschätzung der Folgen von Politikänderungen auf die Agrarproduktion der EU ist derzeit in einigen Bereichen noch unbefriedigend. Dies betrifft insbesondere Einkommensänderungen und das mögliche Anpassungsverhalten auf Betriebsebene.

Bisherige Analysen zu den Auswirkungen alternativer Milchmarktpolitiken für die EU-15 richten sich schwerpunktmäßig auf die Marktebene (BOUAMRA-MECHMACHE, DARTIGUES und Requillart, 2001; Banse, 2001; Salamon, 2001; Helming und Peerlings, 2001; Benjamin, Gohin und Guyomard, 1999; Colman et al., 2002; Arfini et al., 2002; HenNESSY und FINGLETON et al., 2000; JANSSON, 2002, HÜTTEL et al., 2005, ISERMEYER et al., 2006). Studien, die auf der einzelbetrieblichen Ebene ansetzen, beschränken sich im Wesentlichen auf Auswertungen in einzelnen Mitgliedstaaten und basieren auf unterschiedlichen Methoden (KLEINHANSS et al., 2002; HEMME/IFCN, 2001; JAYET, 2002, ISERMEYER et al., 2006). Die Analysen auf der Grundlage der Buchführungsergebnisse des Informationsnetzes landwirtschaftlicher Buchführungen (INLB, englische Bezeichnung FADN ${ }^{2}$ ) beziehen sich entweder nur auf ein Land (REINHARD, 1999; ColmAN, 2001; ARFINI, 2001/03, ISERMEYER et al., 2006), so dass die Vergleichbarkeit der Ergebnisse nur national gegeben ist, oder sie basieren als Fallstudien lediglich auf einer kleinen und nicht repräsentativen Anzahl von Betrieben (HENNESSY, 2000/01). Insbesondere besteht die Notwendigkeit, zusätzliche Kenntnisse über das landwirtschaftliche Anpassungsverhalten bereitzustellen, z. B. zur Modellierung praxisnaher und repräsentativer Anpassungsstrategien als Reaktion auf eine veränderte Agrarpolitik (vgl. JACOBS, 1998).

Deutsche Bezeichnung: Halbzeitbewertung der Agenda 2000.

2 Farm Accountancy Data Network, englische Bezeichnung für INLB. 
Zur Darstellung der Einkommenslage in der Landwirtschaft wird das FADN bereits seit 1965 eingesetzt und weiterentwickelt. Seit mehreren Jahren wird das FADN auch für die Politikfolgenabschätzung verwendet. Die FADN-Datensätze sind EU-weit harmonisiert und repräsentativ. Die jährlichen Erhebungen der FADN-Daten ermöglichen im Fall identischer Betriebe auch die Analyse der Betriebsergebnisse über mehrere Jahre. Diesen Vorteilen stehen jedoch auch Nachteile gegenüber. So fehlt dem FADN-Datensatz die erforderliche Datentiefe, insbesondere in Bezug auf das Mengengerüst. Außerdem sind die regionalen Durchschnittsergebnisse des FADN für eine nach Betriebstypen und Produktionssystemen differenzierte Analyse häufig nicht ausreichend.

Einen möglichen Ansatz zur Verringerung der Nachteile des FADN-Datensatzes bieten die Datensätze typischer Modellbetriebe aus dem International Farm Comparison Network (IFCN). Diese Daten basieren auf Buchführungsabschlüssen, Betriebszweigabrechnungen und dem Expertenwissen von Beratern und Landwirten. Sie sind länderübergreifend harmonisiert und ermöglichen aufgrund der Erfassungstiefe die Abschätzung betrieblicher Anpassungsreaktionen bei veränderten Rahmenbedingungen. Die Datensätze sind aufgrund der geringen Anzahl von Betrieben pro Land jedoch nicht repräsentativ.

Keine der Datenquellen reicht also allein für eine umfassende Analyse aus. Daher bietet es sich an, die Vorteile beider Datensätze zu kombinieren, um eine verbesserte Aussagekraft einzelbetrieblicher Wirtschaftlichkeits- und Politikanalysen auf europäischer Ebene zu erreichen.

\subsection{Zielsetzung}

Vor diesem Hintergrund ist es das Ziel dieser Arbeit, ein Konzept zur Verknüpfung der einzelbetrieblichen Datensätze aus FADN und IFCN zu entwickeln. Dabei soll insbesondere untersucht werden,

- wie viele Betriebe/Milchmenge die bisher als typisch ausgewählten IFCN-Betriebe repräsentieren und wie sie hinsichtlich ihrer Rentabilität statistisch einzuordnen sind. Hierfür werden in erster Linie der FADN-Datensatz herangezogen, aber auch andere Statistiken.

- wie der FADN-Datensatz nutzbar gemacht werden kann für eine künftig verbesserte Betriebsauswahl für das IFCN-Konzept.

- ob sich der FADN-Datensatz mit Hilfe des detaillierten IFCN-Datensatzes so modifizieren lässt, dass er für betriebliche Simulationsrechnungen (z. B. mit dem Modell TIPI-CAL) ${ }^{3}$ nutzbar gemacht werden kann. Das wird anhand eines Beispiels aufgezeigt, ließe sich aber künftig auch auf viele Betriebe übertragen. 


\subsection{Vorgehensweise}

Nach der Einführung in die Problemstellung und Zielsetzung dieser Arbeit wird in Kapitel 2 erörtert, warum sich der Milchmarkt für den Methodenvergleich und für die Integration von FADN- und IFCN-Konzept anbietet. Dies erfolgt anhand eines Überblicks über die Bedeutung und Struktur der europäischen Milchwirtschaft. Angesichts der agrarpolitisch hohen Bedeutung des EU-Milchmarktes und der zur Verfügung stehenden Datenbasis soll das Verknüpfungskonzept am Beispiel von Milchviehbetrieben in ausgewählten EURegionen angewendet werden.

In Kapitel 3 wird anschließend ein detaillierter Überblick über den Anwendungsstand quantitativer Politikfolgenanalysen am Beispiel des EU-Milchmarktes gegeben. Der Modellüberblick dient dazu, die Problemstellung dieser Arbeit zu verdeutlichen und ein geeignetes Modell für das Vorhaben dieser Arbeit auszuwählen. Im Kern handelt es sich bei der Modellbeschreibung um den methodischen Ansatz, den Analyseschwerpunkt, die regionale Differenzierung, die Datengrundlage und um den Zeitraum der Betrachtung. Einführend werden zunächst die Entwicklung der Modellierung zur Politikfolgenabschätzung in Deutschland beschrieben und die zugrunde liegenden Ansätze dargestellt. Danach erfolgt eine Erörterung ausgewählter europäischer Analysen zum Mid-term Review im Zeitraum 1999 bis 2002, um Eignung und Potenziale der jeweiligen Ansätze aufzuzeigen. Anschließend wird ein geeigneter Modellansatz zur Anwendung des Verknüpfungskonzeptes ausgewählt.

Einen Überblick über die verfügbaren Datengrundlagen, die bei den quantitativen Politikfolgenabschätzungen am Beispiel des EU-Milchmarktes vornehmlich Verwendung fanden, wird in Kapitel 4 gegeben. Es werden die gesetzlich vorgeschriebenen Erhebungen in Deutschland und in der EU wie auch bedeutende ergänzende Datensätze erläutert, die im Rahmen der vorgestellten Modellanalysen zum Mid-term Review zur Anwendung kamen. Das Hauptaugenmerk liegt dabei auf den in Kapitel 3 entwickelten Kriterien des Anforderungsprofils. Im Hinblick auf diese Kriterien erfolgt die Auswahl von zwei geeigneten Datenquellen für das Vorhaben dieser Arbeit. Die ausgewählten Datengrundlagen, FADN und IFCN, werden anschließend ausführlich erläutert.

In Kapitel 5 wird erläutert, wie FADN und IFCN für Analysen zur Liberalisierung des EU-Milchmarktes eingesetzt worden sind. Dazu werden zwei Analysekonzepte vorgestellt: a) der Einsatz für die Produktionskostenanalyse und b) der Einsatz für die modellgestützte Politikfolgenabschätzung. Dabei werden die Begrenzungen der jeweiligen Datensätze aufgezeigt und anschließend Schlussfolgerungen für eine kombinierte Anwendung gezogen.

In Kapitel 6 wird die Verknüpfung von FADN- und IFCN-Datensätzen zur Einordnung der IFCN-Betriebe erörtert. Zunächst wird das Verknüpfungskonzept im Überblick vorgestellt. Anschließend wird auf die einzelnen methodischen Elemente des Verknüpfungsan- 
satzes detailliert eingegangen. Dazu zählen die Selektion von FADN-Vergleichsbetrieben mit Hilfe der euklidischen Distanz, die statistische Einordnung typischer IFCN-Betriebe und die Schätzung der FADN-Produktionskosten der Milcherzeugung. Im Folgenden wird die Leistungsfähigkeit des vorgestellten Verknüpfungskonzeptes überprüft und bewertet. Als erstes werden geeignete Untersuchungsregionen und -betriebe (aus dem IFCN) für die Anwendung des Verknüpfungskonzeptes ausgewählt. Anschließend werden die Resultate aus der Selektion ähnlicher FADN-Vergleichsgruppen aufgezeigt. Dabei werden, im Hinblick auf das erste Teilziel dieser Arbeit, für die IFCN-Untersuchungsbetriebe die repräsentierte Anzahl der Betriebe in der Grundgesamtheit und die repräsentierte Milchmenge (absolut und relativ) ermittelt. Zur Einordnung der IFCN-Betriebe in die FADNVerteilung werden zwei FADN-Vergleichsgruppen mit einer hohen Ähnlichkeit zu den IFCN-Untersuchungsbetrieben gewählt. Anschließend wird die Einordnung der IFCNBetriebe in Bezug auf den wirtschaftlichen Erfolg (Rentabilität) vorgestellt, diskutiert und Schlussfolgerungen aus der ersten Verknüpfungsansatz abgeleitet. Zum Abschluss wird, im Hinblick auf das zweite Teilziel dieser Arbeit, ein neues Verfahren zur Auswahl von typischen IFCN-Betrieben aus dem FADN-Datensatz vorgestellt.

In Kapitel 7 wird das Konzept zur Modifizierung der FADN-Daten zur Simulation im Modell TIPI-CAL erläutert. Zunächst wird das Verknüpfungskonzept im Überblick vorgestellt. Anschließend wird die methodische Grundlage beschrieben, mit der die FADNDaten für Projektionsrechnungen ergänzt, auf Plausibilität überprüft und unter Beibehaltung der Annahmen für den IFCN-Betrieb (Preise, Mengen, betriebliche Anpassungsstrategie) in die Zukunft projiziert werden (u. a. Koeffizientenschätzansatz). Im Folgenden werden die Ergebnisse aus der zweiten Verknüpfungsansatz vorgestellt. Zunächst wird die Aufbereitung des FADN-Datensatzes zur Simulation im Modell TIPI-CAL beschrieben. Zudem werden die Szenarien und Betriebsstrategien für die Politikfolgenabschätzung bestimmt. Berechnet werden exemplarisch die Auswirkungen des MTR-Beschlusses ${ }^{4}$ und eines fiktiven Milchquotenausstiegs für den Simulationszeitraum 1999-2008. Das Referenzszenario, das zum Vergleich der beiden Politiken genutzt wird, beinhaltet die Beibehaltung der „Agenda 2000“-Politik. Die erzielten Ergebnisse mit dem modifizierten FADNDatensatz werden mit den Ergebnissen des entsprechenden IFCN-Betriebes verglichen. Es wird diskutiert, in wieweit die FADN-Betriebsdaten für die Simulation im Modell TIPICAL nutzbar gemacht werden konnten. Dabei werden Möglichkeiten, Grenzen und zukünftige Perspektiven der Aufbereitung und Verknüpfung der Datensätze FADN und IFCN in der Politikfolgenanalyse aufgezeigt.

Abschließend erfolgt in Kapitel 8 die Zusammenfassung dieser Arbeit.

Mid-term Review-Beschluss aus dem Monat Juni 2003. 


\section{Struktur der europäischen Milchwirtschaft}

Der Milchsektor ist für die Europäische Union (EU) aus mehreren Gründen von großer Bedeutung. Die wichtigsten Gründe sollen im Folgenden kurz erläutert werden.

Milch ist mit ungefähr $14 \%$ des gesamten landwirtschaftlichen Produktionswertes das bedeutendste Produkt im Agrarsektor. Im Jahr 2004 wurde von den landwirtschaftlichen Betrieben Milch im Wert von ca. 43 Mrd. € erzeugt (EU-Kommission, 2007). Der Umsatz des Milchverarbeitungssektors betrug in demselben Jahr 117 Mrd. €. Die EU ist ein Hauptakteur auf dem Weltmarkt für Milcherzeugnisse und bei vielen Milcherzeugnissen führender Exporteur - zum Beispiel bei Käse.

Der Stellenwert der Milch wird auch dadurch unterstrichen, dass ausnahmslos alle Mitgliedstaaten der EU Milch erzeugen. Allerdings unterscheiden sich die Mitgliedstaaten stark im Hinblick auf die Produktionsmethoden, Milchleistungen, Produktqualität, Betriebsstrukturen und Einkommen. Durch die Osterweiterung wurden diese Unterschiede zwischen den Mitgliedsländern der EU noch verstärkt.

Für den EU-Milchmarkt ist eine starke politische Einflussnahme kennzeichnend. 1984 wurde die Milchquotenregelung als zeitlich begrenzter Kontrollmechanismus eingeführt, um hohe Überschüsse in der EU-Milchproduktion zu verhindern. Der EU-Milchmarkt ist protegiert gegenüber dem Weltmarkt durch Zölle, Interventionspreise und Exporterstattungen. Er befindet sich derzeit jedoch in einer Phase grundlegender Änderungen. Der Mid-term Review ${ }^{1}$ (MTR)-Beschluss aus dem Jahr 2003, die EU-Ost-Erweiterung und die laufenden WTO-Verhandlungen werden die Rahmenbedingungen der Landwirtschaft in der EU weiter grundlegend bestimmen. Die im Rahmen des MTR beschlossene Milchmarktreform bewirkt eine deutliche Verringerung der Interventionspreise für Butter und Magermilchpulver. Darüber hinaus wurden die Einführung von entkoppelten Prämien und die Beibehaltung des Milchquotensystems bis zum 31.03.2015 beschlossen. Laut Health Check geht die europäische Kommission aber davon aus, dass die Quotenregelung zum 31.03.2015 endgültig ausläuft, so dass sich die Rahmenbedingungen für den EUMilchmarkt dann grundlegend ändern werden (EU-KOMMISSION, 2008).

Auf internationaler Ebene implizieren die Beschlüsse aus der laufenden DOHAEntwicklungsrunde der WTO-Verhandlungen und die landwirtschaftlichen Übereinkommen aus der Uruguay Runde (URAA) eine Begrenzung der Einfuhrbeschränkungen und den Abbau von Exporterstattungen. Jede dieser Änderungen hat bedeutende Auswirkungen auf den gesamten EU-Milchsektor. 
Im Folgenden wird ein regional differenzierter Überblick über die EU-Milchwirtschaft (EU-15) gegeben. Er basiert maßgeblich auf Daten von Eurostat, dem statistischen Bundesamt, der ZMP, Faostat, dem Milk Development Council (MDC) und dem USDA- „Foreign Agricultural Service“. Ergänzend wird eine EU-Milchmarktstudie von CHATELLIER und JACQUERIE (2003) herangezogen, die auf europäischen Testbetriebsdatensätzen (FADN) basiert.

\section{Milchmarkt}

Die Kuhmilchproduktion in der EU-15 beläuft sich auf 121 Mio. Tonnen (EUROstAT, 2007), davon werden ca. 114 Mio. Tonnen von den Molkereien verarbeitet. In Tabelle 2.1 ist die Kuhmilcherzeugung je EU-Mitgliedstaat von 1998 bis 2005 im Überblick aufgezeigt. Die Milchproduktion der EU-15 hat sich im Wirtschaftjahr 2005/2006 im Vergleich zu 2004/2005 um ca. 0,25 \% reduziert. Die leichte Verringerung der EU-Milcherzeugung trotz Ausweitung der Milchquotenmenge in der EU-15 (USDA FoREIGN AGRICULTURAL SERVICE, 2006) hat unterschiedliche Ursachen: Neben Witterungseinflüssen zeigt auch die Umsetzung der Luxemburg-Reform (die Entkoppelung der Milchprämien von der Produktion) erste Effekte.

Im ersten Monat des Wirtschaftsjahres 2007/2008 hat sich allerdings die Milchproduktion der EU-15 im Vergleich zu 2006/2007 um ca. 1,5 \% erhöht. Im Februar 2008 war der größte Produktionszuwachs von 6,6 \% im Vergleich zum Februar des Vorjahres zu verzeichnen. Dies hat dazu beigetragen, dass die EU-25 ca. 131,6 Mio. Tonnen Milch an die Molkereien in 2007/08 lieferte, ca. 337.000 Tonnen (0,26 \%) mehr als im Wirtschaftsjahr 2006/07. Die Landwirte in Deutschland, den Niederlanden, Spanien, Polen und insbesondere in Frankreich scheinen alle auf die Signale des Marktes durch Erhöhung ihrer Produktion reagiert zu haben (z. B. durch Verlängerung der Laktationsperiode, durch höheren Einsatz von Kraftfutter, Verschiebung der Schlachttermine etc.). Das Vereinigte Königreich und Italien sind die einzigen Länder unter den größten Milchproduzenten der EU, die einen Rückgang der Milchlieferungen zu verzeichnen haben.

Zu den größten Milchproduzenten innerhalb der EU zählen Deutschland, Frankreich, Vereinigtes Königreich, Niederlande und Italien. Die Referenzmengen je EU-Mitgliedstaat (EU-15) sind im Anhang in Tabelle A2.1 dargestellt. 
Tabelle 2.1: Entwicklung der Kuhmilchproduktion in Europa

\begin{tabular}{|c|c|c|c|c|c|c|c|c|}
\hline & 1998 & & & 2001 & 2002 & 2003 & 2004 & 2005 \\
\hline \multicolumn{9}{|c|}{ Tausend Tonnen ${ }^{1)}$} \\
\hline \multicolumn{9}{|l|}{ EU - 15} \\
\hline Deutschland & 28.329 & 28.334 & 28.332 & 28.191 & 27.874 & 28.533 & 28.245 & 28.622 \\
\hline Frankreich & 24.585 & 24.614 & 24.734 & 24.879 & 25.173 & 24.590 & 24.344 & 23.857 \\
\hline Italien & 11.250 & 11.072 & 10.774 & 10.793 & 10.879 & $10.793^{2)}$ & $10.795^{2)}$ & $10.805^{2)}$ \\
\hline Niederlande & 10.995 & 11.174 & 11.155 & 10.970 & 10.677 & 11.074 & 10.905 & 10970 \\
\hline Belgien & 3.418 & 3.382 & 3.425 & 3.357 & 3.160 & 3.127 & $3.149^{2)}$ & $3.034^{2)}$ \\
\hline Luxemburg & 266 & 267 & 267 & 269 & 270 & 265 & 269 & 269 \\
\hline Vereinigtes Königreich & 14.632 & 15.013 & 14.489 & 14.707 & 14.869 & 15.010 & 14.630 & 14.651 \\
\hline Republik Irland & 5.200 & 5.225 & 5.265 & 5.445 & 5.293 & 5.416 & 5.373 & 5.184 \\
\hline Dänemark & 4.668 & 4.656 & 4.719 & 4.553 & 4.590 & 4.671 & 4.568 & 4.600 \\
\hline Griechenland & 749 & 775 & 789 & 778 & 758 & 768 & 770 & $770^{2)}$ \\
\hline Spanien & 5.980 & 6.172 & 5.900 & 6.520 & 6.611 & 6.636 & 6.635 & 6.600 \\
\hline Portugal & 1.850 & 2.040 & 2.060 & 1.983 & 2.103 & 1.952 & 2.010 & $2.100^{2)}$ \\
\hline Österreich & 3.043 & 3.132 & 3.233 & 3.300 & 3.292 & 3.230 & 3.137 & 3.130 \\
\hline Finnland & 2.447 & 2.475 & 2.524 & 2.530 & 2.532 & 2.472 & 2.449 & 2.437 \\
\hline Schweden & 3.331 & 3.352 & 3.348 & 3.339 & 3.274 & 3.253 & 3.275 & 3.275 \\
\hline \multicolumn{9}{|l|}{ NMS 10} \\
\hline Estland & 730 & 626 & 630 & 684 & 612 & 612 & 652 & $672^{2)}$ \\
\hline Zypern & 134 & 133 & 147 & 142 & 154 & 164 & 145 & $135^{2)}$ \\
\hline Tschechische Republik & 2.798 & 2.818 & 2.789 & 2.782 & 2.809 & 2.725 & 2.673 & 2.770 \\
\hline Lettland & 948 & 797 & 823 & 848 & 806 & $758^{2)}$ & $837^{2)}$ & $726^{2)}$ \\
\hline Litauen & 1.930 & 1.714 & 1.725 & 1.730 & 1.771 & 1.796 & 1.890 & 1.930 \\
\hline Ungarn & 2.045 & 2.045 & 2.081 & 2.080 & 3.068 & 1.988 & 1.895 & 1.849 \\
\hline Malta & 2 & 0 & 48 & 47 & 45 & 41 & 43 & $42^{2)}$ \\
\hline Polen & 12.596 & 12.273 & 11.889 & 11.884 & 11.873 & 11.891 & 11.478 & 11900 \\
\hline Slowenien & 599 & 634 & 649 & 653 & 728 & $755^{2)}$ & $815^{2)}$ & $866^{2)}$ \\
\hline Slowakei & 1.142 & 1.105 & 1.099 & 1.114 & 1.163 & $1189^{2)}$ & $1.231^{2)}$ & $1.268^{2)}$ \\
\hline \multicolumn{9}{|l|}{ Geamt } \\
\hline EU-25 & 143.667 & 143.827 & 142.892 & 143.578 & 144.383 & 143.710 & 142.212 & 142.462 \\
\hline EU-15 & 120.743 & 121.683 & 121.013 & 121.614 & 121.355 & 121.790 & 120.554 & 120.304 \\
\hline NMS 10 & 22.924 & 22.144 & 21.879 & 21.964 & 23.027 & 21.920 & 21.657 & 22.158 \\
\hline
\end{tabular}

1) Ohne Milch zur Fütterung des Jungviehs.

Produktion $=$ Lieferung an Molkereien + Direktabsatz + an Vieh verfütterte Milch + Konsum Betriebshaushalt.

2) Schätzung.

Quelle: MDC Datum (Milk Development Council), Eurostat, 2006. 
Trotz bestehender Milchquotenregelung verzeichnete der EU-Milchsektor stets Überschüsse. Tabelle 2.2 gibt die gesamte Milchanlieferung, den Verbrauch und den Selbstversorgungsgrad wieder. Der Selbstversorgungsgrad beläuft sich auf durchschnittlich 115\%. Aufgrund der verstärkten Nachfrage nach Milch- und Milchprodukten u. a. aus Russland und China hat sich im Jahr 2007 eine Knappheit eingestellt, deren Dauer noch nicht abzuschätzen ist. Der Selbstversorgungsgrad reduzierte sich in 2007 auf 108 \%. Aufgrund des starken Anstiegs der Milchpreise auf dem Weltmarkt werden seit Juni 2007 keine Exporterstattungen für Milchprodukte mehr gezahlt. Aufgrund der Erhöhung der Milchquotenmenge in der EU ist in den nächsten Jahren ein wieder ansteigender Selbstversorgungsgrad absehbar.

Tabelle 2.2: $\quad$ Kuhmilchbilanz der Europäischen Union

\begin{tabular}{|c|c|c|c|c|c|c|}
\hline & $\begin{array}{r}2002 \\
\text { EU-15 }\end{array}$ & $\begin{array}{r}2003 \\
\text { EU-15 }\end{array}$ & $\begin{array}{r}2004 \\
\text { EU-25 }\end{array}$ & $\begin{array}{l}2005^{1)} \\
\text { EU-25 }\end{array}$ & $\begin{array}{l}2006^{1)} \\
\text { EU-25 }\end{array}$ & $\begin{array}{l}2007^{1)} \\
\text { EU-27 }\end{array}$ \\
\hline Milchanlieferung (Mio.t) & 115,67 & 116,23 & 131,0 & 132,6 & 131,7 & 133,0 \\
\hline + Einfuhr in Milchäquivalent & 4,49 & 4,43 & 3,7 & 3,3 & 3,0 & 3,2 \\
\hline - Ausfuhr in Milchäquivalent & 12,65 & 13,47 & 14,9 & 13,5 & 12,1 & 13,2 \\
\hline $\begin{array}{l}\text { - Bestandsveränderung in } \\
\text { Milchäquivalent }\end{array}$ & 0,89 & 0,48 & $-2,5$ & $-0,8$ & $-0,3$ & 0,0 \\
\hline $\begin{array}{l}\text { Verbrauch in Milchäquivalent (Mio. t) } \\
\text { davon }\end{array}$ & 106,62 & 106,70 & 122,3 & 123,2 & 122,9 & 122,9 \\
\hline - Verbrauch zu Marktpreisen & 96,52 & 96,18 & 111,4 & 112,3 & 114,7 & 122,8 \\
\hline - Verbrauch mit Beihilfen & 10,10 & 10,52 & 10,9 & 10,9 & 8,2 & 0,2 \\
\hline Selbstvers orgungsgrad (\%) & 108 & 109 & 107 & 108 & 107 & 108 \\
\hline $\begin{array}{l}\text { - desgleichen um den Verbrauch } \\
\text { mit Beihilfen bereinigt }\end{array}$ & 117 & 118 & 115 & 115 & 113 & 108 \\
\hline
\end{tabular}

Von der gesamten in der EU-15 erzeugten Milch wird ca. ein Drittel zu Butter verarbeitet (EU-Kommission, 2006). Der Anteil an Butter reduzierte sich seit 1973 fortlaufend, während sich die Produktion von Käse, Sahne und Milchpulver in demselben Zeitraum kontinuierlich erhöhte. Der Anteil Frischmilcherzeugnisse blieb stabil. Die Verarbeitung und der Handel von Käse, Butter, Magermilchpulver und Vollmilchpulver sind im Anhang in Tabelle A2.2 bis A2.5 dargestellt.

Die Verarbeitung von Frischmilch zu Käse ist die profitabelste Aktivität der EUMilchverarbeiter (Stand 2007, USDA Foreign Agricultural Service, 2006). Auch bei geringeren Milchanlieferungen folgte die Käseproduktion 2006 der steigenden Nachfrage. Wegen der Ausweitung der Käseproduktion stand für andere Produkte weniger Rohmilch zur Verfügung, die Produktion von Butter und Magermilchpulver (NFDN - Non Fat Dry 
Milk) nahm ab. Die reduzierte Produktion von Butter in 2006 führte zu verringerten Butterexporten.

In Tabelle 2.3 sind die Im- und Exporte von Milch- und Milcherzeugnissen dargestellt. Im Zeitablauf von 1998 bis 2004 sind die Importe von Frischmilch und allen anderen aufgeführten Milcherzeugnissen in die EU angestiegen. Bei Frischmilch sind die Importe um ca. $10 \%$ und bei Käse um ca. 30 \% angestiegen. Die Exporte von Kondensmilch, Vollmilchpulver und Molke verringerten sich von 1998 bis 2004, dagegen schlagen aber die Exportzunahmen bei den anderen Milcherzeugnissen und der Frischmilch stärker zu Buche.

Vor dem Hintergrund der WTO-Verhandlungen sind die Abschottung des EUMilchmarktes und die Förderung der EU-Milcherzeugnisse auf dem Weltmarkt mittels Exporterstattungen nicht unbegrenzt aufrecht zu erhalten. Bei der in Tabelle 2.3 beschriebenen Ausweitung sowohl der Importe als auch der Exporte und bei der angestrebten Handelsliberalisierung ist anzunehmen, dass internationaler Handel und internationale Wettbewerbsfähigkeit für die künftige Milchproduktion an Bedeutung gewinnen werden.

Tabelle 2.3: Übersicht über den Handel von Milcherzeugnissen (EU-15)

\begin{tabular}{|c|c|c|c|c|c|c|c|}
\hline & 1998 & 1999 & 2000 & $\begin{array}{c}2001 \\
\text { Tonnen }\end{array}$ & 2002 & 2003 & 2004 \\
\hline \multicolumn{8}{|l|}{ IMPORTE } \\
\hline Frischmilch & 5.252 .538 & 5.434 .848 & 5.500 .704 & 5.265 .411 & 5.100 .124 & 6.003 .998 & 5.867 .716 \\
\hline Sahne & 493.578 & 578.360 & 565.300 & 515.062 & 544.034 & 534.830 & 584.207 \\
\hline Butter und Butteröl $^{a}$ & 684.187 & 696.169 & 723.169 & 697.050 & 710.274 & 773.551 & 767.053 \\
\hline Käse & 1.861 .614 & 1.994 .318 & 2.051 .957 & 2.293.221 & 2.126 .365 & 2.411 .272 & 2.498.311 \\
\hline Kondensmilch & 534.968 & 496.346 & 561.047 & 528.467 & 501.446 & 569.400 & 547.906 \\
\hline Milchpulver (WMP) $^{\text {b }}$ & 254.543 & 250.810 & 259.390 & 244.645 & 265.661 & 259.870 & 275.111 \\
\hline Magermilchpulver (SMP) & 519.847 & 597.520 & 630.553 & 529.306 & 539.760 & 584.338 & 633.785 \\
\hline Molkepulver & 602.723 & 580.808 & 632.627 & 680.653 & 632.718 & 678.219 & 706.309 \\
\hline Flüssige Molke & 535.638 & 549.089 & 628.401 & 737.930 & 583.459 & 603.271 & 613.958 \\
\hline \multicolumn{8}{|l|}{ EXPORTE } \\
\hline Frischmilch & 5.136 .876 & 5.527 .657 & 5.413 .721 & 5.213 .946 & 4.698 .850 & 5.782 .012 & 5.938.276 \\
\hline Sahne & 596.581 & 647.576 & 667.870 & 604.560 & 569.070 & 650.311 & 664.873 \\
\hline Butter und Butteröl $^{a}$ & 754.139 & 696.902 & 688.181 & 712.156 & 686.256 & 859.997 & 886.532 \\
\hline Käse & 2.251 .277 & 2.266 .611 & 2.398 .850 & 2.863 .933 & 2.527 .091 & 2.819 .781 & 2.887 .569 \\
\hline Kondensmilch & 842.715 & 811.895 & 816.466 & 829.053 & 724.853 & 782.310 & 694.347 \\
\hline Milchpulver (WMP) ${ }^{\mathrm{b}}$ & 826.611 & 795.474 & 799.043 & 684.585 & 714.665 & 733.041 & 750.254 \\
\hline Magermilchpulver (SMP) & 638.826 & 778.850 & 916.158 & 593.726 & 554.579 & 721.723 & 688.341 \\
\hline Molkepulver & 728.652 & 740.889 & 864.280 & 899.264 & 830.420 & 897.026 & 962.545 \\
\hline Flüssige Molke & 703.599 & 716.948 & 1.114 .845 & 1.096 .267 & 354.842 & 435.226 & 453.704 \\
\hline
\end{tabular}

a) inkl. Molkereidistribution

b) inkl. teilentrahmtes Milchpulver

Quelle: MDC Datum (Milk Development Council), Eurostat. 


\section{Struktur der Milchviehbetriebe}

Der EU-Milchsektor ist im Wesentlichen durch folgende Strukturveränderungen gekennzeichnet:

- In den meisten Ländern der EU-15 hat sich der Gesamtbestand an Milchkühen verringert. Die durchschnittlichen Herdengrößen sind dagegen kontinuierlich gewachsen (siehe Tabelle 2.4).

- Die Anzahl der Milchvieh haltenden Betriebe hat sich im Zeitraum von 1998 bis 2005 zum Teil deutlich verringert (vgl. Tabelle 2.5). Die Anzahl größerer Betriebe hat dagegen zugenommen. .

- Die durchschnittliche Milchleistung ist in allen Ländern der EU-15 angestiegen (siehe Tabelle 2.6).

Tabelle 2.4: Entwicklung der Anzahl Milchkühe und der durchschnittlichen Herdengröße in der EU (2000 bis 2004) ${ }^{1)}$

\begin{tabular}{|c|c|c|c|c|c|c|c|c|c|c|}
\hline & \multicolumn{4}{|c|}{ Milchkühe } & \multicolumn{6}{|c|}{ Durchschnittliche Herdengröße } \\
\hline & 2000 & 2001 & 2002 & 2003 & 2004 & 2000 & 2001 & 2002 & 2003 & 2004 \\
\hline Belgien & 615.900 & 625.100 & 602.300 & 585.400 & 568.600 & 35 & 35 & 35 & 35 & 36 \\
\hline Dänemark & 644.000 & 628.000 & 613.000 & 589.000 & 569.000 & 66 & 64 & 76 & 80 & 86 \\
\hline Deutschland & 4.569 .800 & 4.548 .600 & 4.427 .200 & 4.372 .000 & 4.285 .100 & 35 & 36 & 37 & 37 & 37 \\
\hline Griechenland & 180.000 & 172.300 & 151.600 & 149.300 & : & 14 & 16 & 16 & 17 & $:$ \\
\hline Spanien & 1.151 .000 & 1.182 .000 & 1.154 .200 & 1.118 .400 & 1.056 .900 & 20 & 23 & 25 & 27 & 28 \\
\hline Frankreich & 4.412 .800 & 4.197.200 & 4.133.800 & 4.026 .200 & 3.947 .000 & 34 & 34 & 35 & 35 & 36 \\
\hline Irland & 1.152 .800 & 1.148 .000 & 1.128 .700 & 1.135 .700 & 1.121 .800 & 40 & 41 & 42 & 45 & 47 \\
\hline Italien & 1.771 .000 & 2.077 .600 & 1.910 .900 & 1.913 .400 & : & 24 & 33 & 32 & 33 & : \\
\hline Niederlande & 1.571 .700 & 1.605 .900 & 1.541 .100 & 1.535 .200 & 1.527 .900 & 47 & 62 & 62 & 64 & 66 \\
\hline Österreich & 621.000 & 598.000 & 589.000 & 557.900 & : & 10 & 10 & 10 & 10 & : \\
\hline Finnland & 357.900 & 351.800 & 343.000 & 328.000 & 317.900 & 16 & 17 & 18 & 18 & 19 \\
\hline Schweden & 425.800 & 425.300 & 417.100 & 402.500 & 403.700 & 35 & 38 & 40 & 41 & 44 \\
\hline Vereinigtes & 2.339 .000 & 2.203 .300 & 2.229 .500 & 2.207 .000 & 2.153 .000 & 90 & 85 & 97 & 103 & 112 \\
\hline Luxemburg & 43.600 & 44.000 & 42.100 & 41.200 & 41.100 & 37 & 40 & 39 & 40 & 41 \\
\hline Portugal & 355.000 & 338.000 & 341.000 & 328.000 & 337.000 & 15 & 16 & 18 & 19 & 21 \\
\hline
\end{tabular}

1) Die Milchviehbetriebe beinhalten alle Betriebe mit Milchkühen, nicht nur spezialisierte Milchviehbetriebe.

Quelle: Eurostat (2007). 
Tabelle 2.5: $\quad$ Entwicklung der Anzahl an Betrieben mit Milchvieh

\begin{tabular}{|c|c|c|c|c|c|c|c|c|}
\hline & 1998 & 1999 & 2000 & 2001 & 2002 & 2003 & $2004^{1)}$ & $2005^{1)}$ \\
\hline Deutschland & 163.600 & 142.900 & 129.892 & 125.100 & 119.800 & 117.100 & 115.200 & 112.000 \\
\hline Frankreich & 140.354 & 134.394 & 128.500 & 123.720 & 119.497 & 115.034 & $109.900^{1)}$ & $105.500^{1)}$ \\
\hline Italien & 90.601 & 80.885 & 74.457 & 63.090 & 59.995 & 57.358 & $55.000^{1)}$ & $48.200^{1)}$ \\
\hline Niederlande & 37.160 & 35.421 & 33.274 & 25.985 & 24.775 & 23.864 & $23.000^{1)}$ & $22.200^{1)}$ \\
\hline Belgien & 18.919 & 18.477 & 17.639 & 17.942 & 17.154 & 16.571 & 15.817 & $14.500^{1)}$ \\
\hline Luxemburg & 1.261 & 1.230 & 1.165 & 1.112 & 1.081 & 1.026 & 1.006 & 990 \\
\hline Vereinigtes Königr & 35.588 & 34.553 & 25.853 & 25.779 & 22.876 & 21.383 & $19.300^{1)}$ & $17.800^{1)}$ \\
\hline Republik Irland & 32.856 & 32.475 & 29.076 & 27.814 & 26.598 & 25.212 & 23.800 & 22.400 \\
\hline Dänemark & 11.373 & 10.570 & 9.737 & 9.737 & 8.062 & 7.400 & 6.600 & 5.950 \\
\hline Griechenland & 15.460 & 13.916 & 12.435 & 11.031 & 9.637 & 8.655 & $7.600^{1)}$ & $6.800^{1)}$ \\
\hline Spanien & 74.230 & 64.776 & 56.379 & 50.362 & 45.905 & 41.149 & $37.300^{1)}$ & $31.100^{1)}$ \\
\hline Potrugal & 40.832 & 31.558 & 23.869 & 20.588 & 19.174 & 17.461 & 16.027 & $14.700^{1)}$ \\
\hline Österreich & 72.148 & 72.358 & 63.949 & 61.191 & 60.786 & 57.268 & 51.031 & 50.000 \\
\hline Finnland & 28.233 & 26.195 & 22.225 & 20.731 & 19.416 & 18.143 & 16.928 & 15.862 \\
\hline Schweden & 14.174 & 13.243 & 12.168 & 11.299 & 10.557 & 9.853 & 9.200 & 8.700 \\
\hline EU-15 & 776.789 & 712.951 & 640.618 & 595.481 & 656.313 & 537.477 & $507.709^{1)}$ & $476.702^{1)}$ \\
\hline
\end{tabular}

1) December census.

Quelle: MDC Datum (Milk Development Council), Eurostat, 2006.

Tabelle 2.6: Durchschnittliche Milchleistung je Kuh und Jahr

\begin{tabular}{|c|c|c|c|c|c|c|}
\hline \multirow[b]{2}{*}{ in $\mathrm{kg} / \mathrm{Jahr}$} & \multirow[b]{2}{*}{2000} & \multicolumn{3}{|c|}{ Milchkühe } & \multirow[b]{2}{*}{2004} & \multirow[b]{2}{*}{$2005^{1)}$} \\
\hline & & 2001 & 2002 & 2003 & & \\
\hline EU-15 & 5.800 & 5.978 & 6.115 & 6.289 & 6.344 & 6.500 \\
\hline Belgien ${ }^{2)}$ & 5.409 & 5.413 & 5.740 & 6.019 & 6.166 & : \\
\hline Dänemark & 7.123 & 7.159 & 7.397 & 7.775 & 7.889 & 8.113 \\
\hline Deutschland & 6.122 & 6.213 & 6.272 & 6.537 & 6.585 & 6.761 \\
\hline Griechenland ${ }^{2)}$ & 4.725 & 4.420 & 4.679 & 5.103 & 5.151 & 5.133 \\
\hline Spanien & 4.964 & 5.551 & 5.704 & 5.893 & 6.158 & 6.307 \\
\hline Frankreich & 5.496 & 5.759 & 5.860 & 5.862 & 5.930 & 6.070 \\
\hline Irland & 4.362 & 4.733 & 4.649 & 4.782 & 4.759 & 4.644 \\
\hline Italien & 4.912 & 4.960 & 5.267 & 5.622 & 5.720 & 5.893 \\
\hline Luxemburg & 5.991 & 6.158 & 6.286 & 6.380 & 6.677 & 6.810 \\
\hline Niederlande & 7.296 & 7.096 & 7.187 & 7.494 & 7.415 & 7.568 \\
\hline Österreich & 5.215 & 5.353 & 5.432 & 5.507 & 5.632 & 5.791 \\
\hline Portugal & 5.787 & 5.723 & 6.199 & 6.280 & 6.452 & $:$ \\
\hline Finnland ${ }^{2)}$ & 6.900 & 7.129 & 7.287 & 7.366 & 7.583 & 7.726 \\
\hline Schweden & 7.829 & 7.989 & 7.852 & 8.073 & 8.107 & 8.055 \\
\hline Vereinigtes Königreich & 6.066 & 6.476 & 6.707 & 6.767 & 6.715 & 6.872 \\
\hline
\end{tabular}

1) Vorläufig bzw. geschätzt. 2) Revidierte Zeitreihe

Quelle: ZMP; nach nationalen Statistiken: EUROSTAT, FAOSTAT data (2006). 
Auch die regionalen Produktionsstrukturen haben sich in der EU-15 deutlich gewandelt. In der Abbildung 2.1 ist die Entwicklung der Betriebsstrukturen in den Ländern der EU-15 aufgezeigt. Daraus wird ersichtlich, dass sich die Anzahl an Betrieben mit weniger als 20 Kühen in allen Ländern deutlich verringert hat, während gleichzeitig eine Tendenz zu größeren Milchkuhherden erkennbar ist. Es ist davon auszugehen, dass es ohne die starke Regulierung des EU-Milchmarktes durch die Milchquotenregelung zu deutlich stärkeren strukturellen Änderungen gekommen wäre. Der regionale Strukturwandel wurde in der EU maßgeblich durch die Quotenregelung beeinflusst, die eine Verschiebung von Produktionsanteilen über Mitgliedstaatengrenzen hinweg seit 1984 weitgehend ausgeschlossen hat. Daher ist es wichtig, sich mit den Konsequenzen einer zunehmenden Liberalisierung des EU-Milchmarktes zu beschäftigen, die aller Voraussicht nach erhebliche Auswirkungen auf die Entwicklung der Betriebsstrukturen in der EU-15 haben wird. 
Abbildung 2.1: $\quad$ Entwicklung der Betriebsstrukturen in der EU-15

Belgien

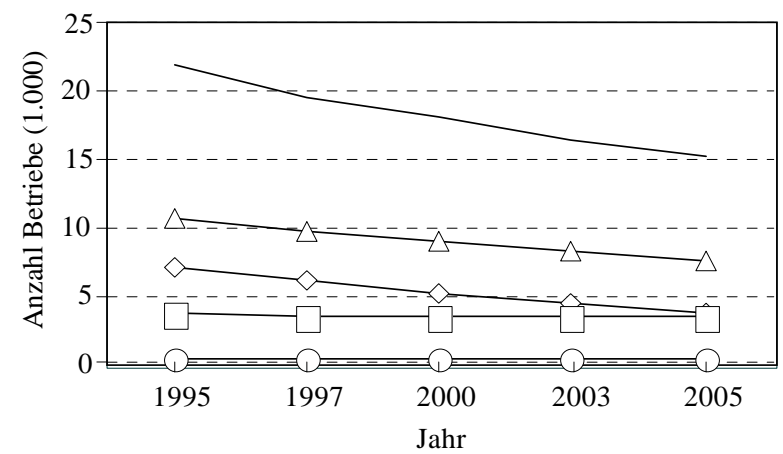

Deutschland

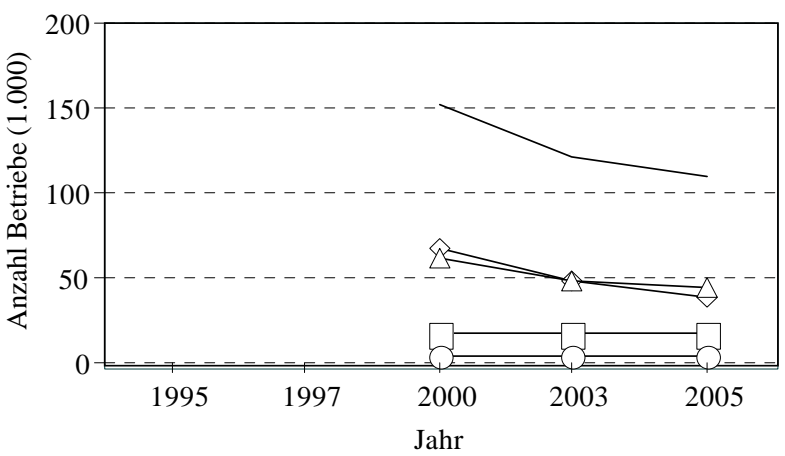

Griechenland

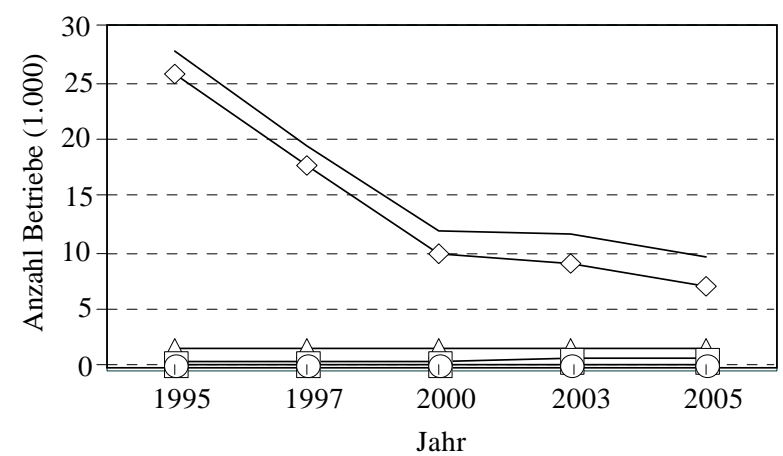

Frankreich

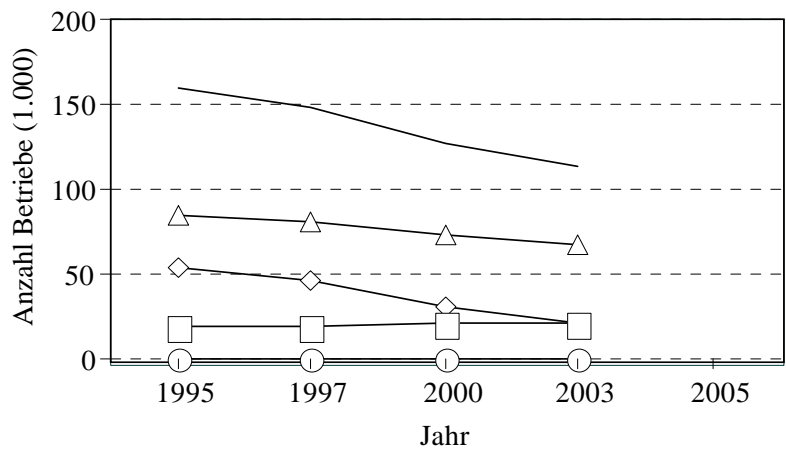

Dänemark

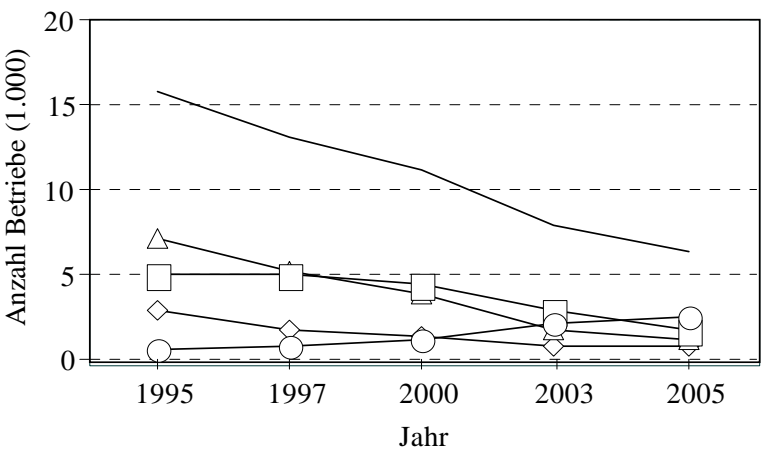

Irland

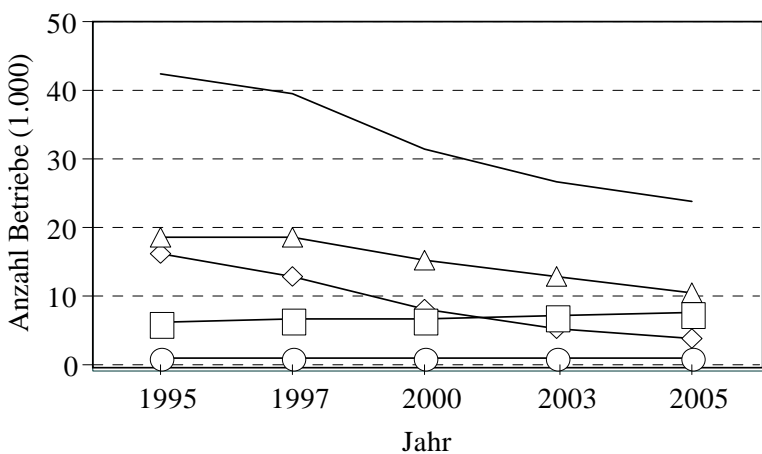

Spanien

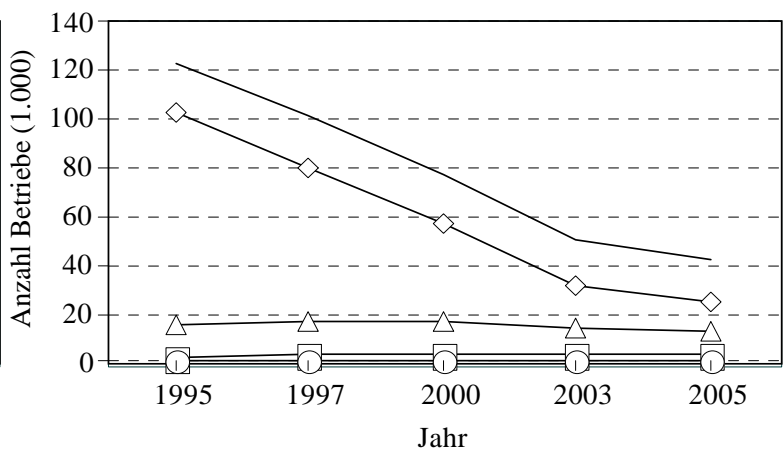

Italien

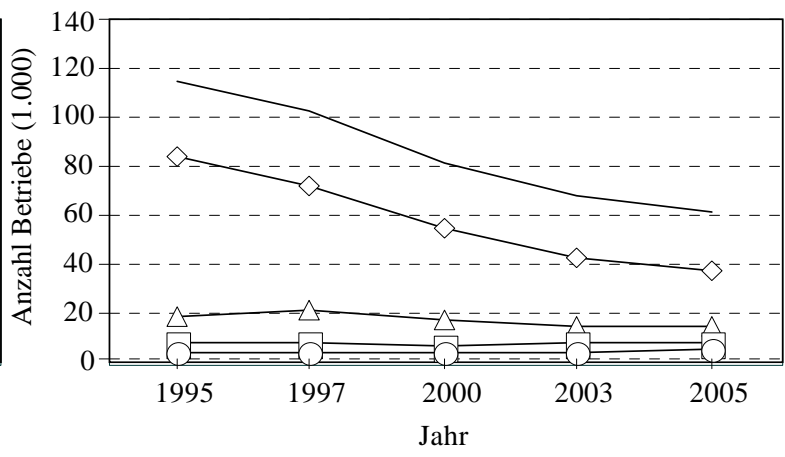

Milchviehbestandsgrößen $\longrightarrow$ Insgesamt -1 bis $19-\square 20$ bis $49-\square 50$ bis $99-\bigcirc$ über 100 
Abbildung 2.1: $\quad$ Entwicklung der Betriebsstrukturen in der EU-15 - Fortsetzung
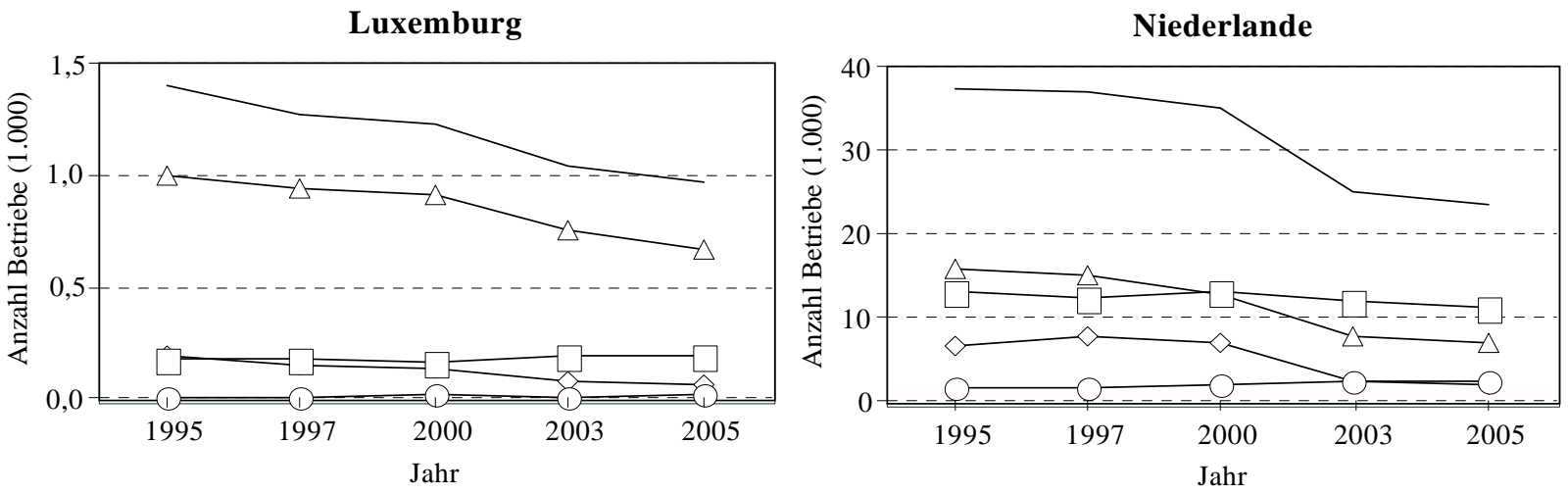

Österreich
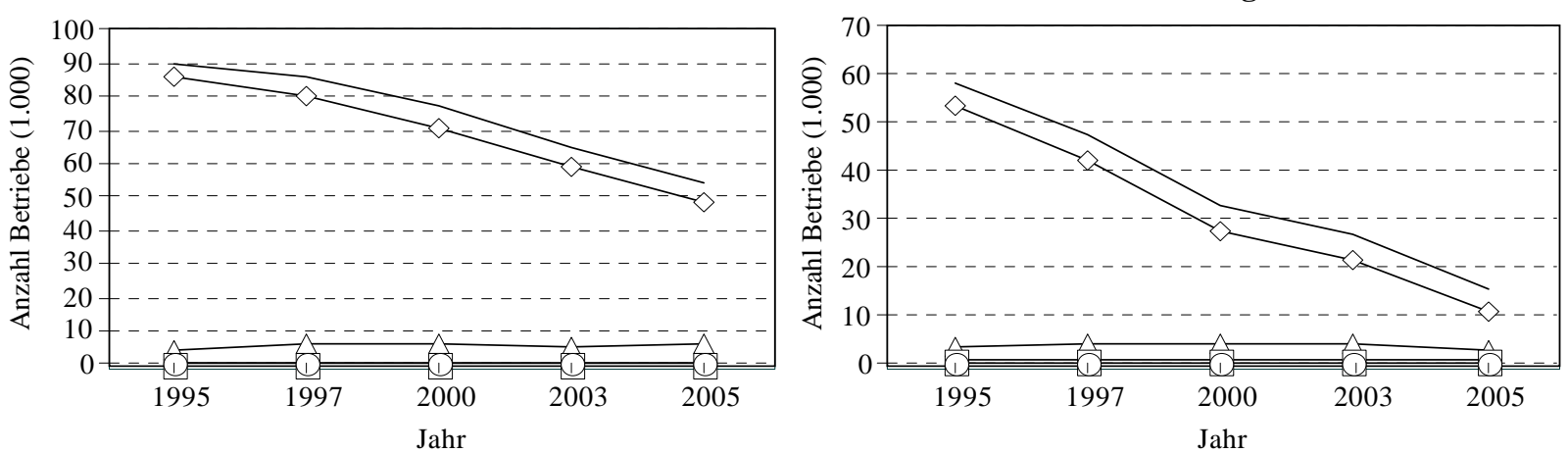

Finnland

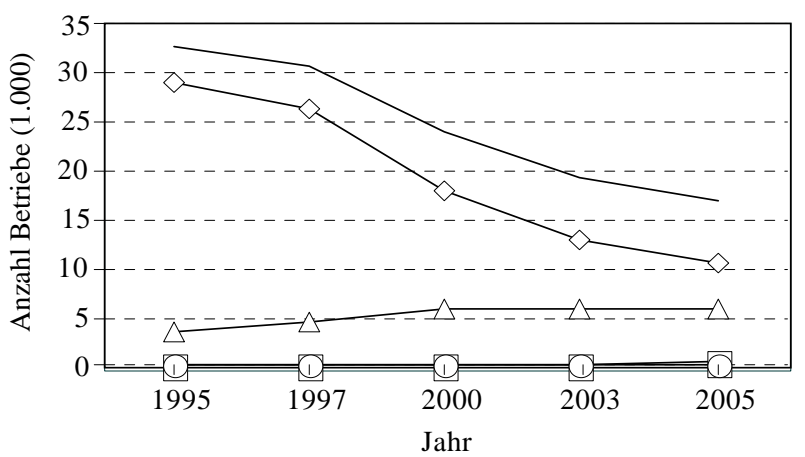

Schweden

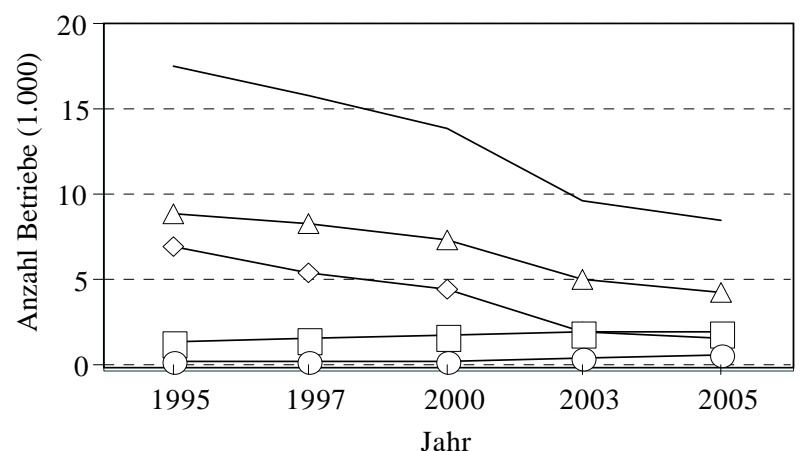

Vereinigtes Königreich
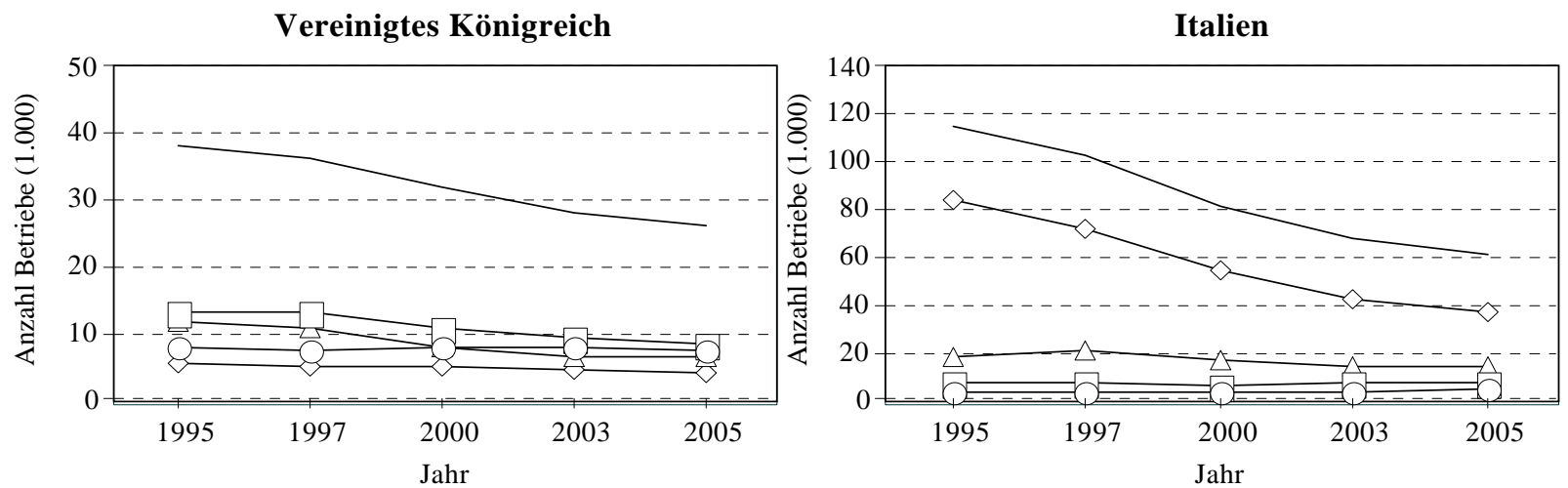

Milchviehbestandsgrößen $\longrightarrow$ Insgesamt $-\longrightarrow 1$ bis $19--20$ bis $49-\square-50$ bis $99-\infty$ über 100 Quelle: Eurostat (2007). 
Der EU-Milchmarkt bietet sich für den Methodenvergleich im Rahmen dieser Arbeit besonders gut an aufgrund

- der Marktlage und der hohen ökonomischen Bedeutung,

- der starken politischen Regulierung, die langfristig nicht aufrechtzuerhalten sein wird,

- der fortschreitenden Liberalisierung des EU-Milchmarktes und der sich abzeichnenden Konsequenzen für die Betriebe,

- offener Fragen zur Reaktion der Betriebe auf die Neuerungen. 


\section{Einzelbetriebliche Modelle in der quantitativen Politikfolgenabschät- zung}

Dieses Kapitel gibt zunächst einen Überblick über die Entwicklung der Modellierung zur Politikfolgenabschätzung in Deutschland und stellt die zugrunde liegenden Ansätze dar. Nachfolgend werden ausgewählte europäische Analysen zur Halbzeitbewertung (englische Bezeichnung: Mid-term Review, MTR) im Zeitraum 1999 bis 2002 vorgestellt, um Eignung und Potenziale der jeweiligen Ansätze aufzuzeigen. Daraus ergeben sich Anforderungen an eine geeignete Modell- und Datengrundlage für die vorliegende Arbeit sowie die Auswahl eines geeigneten Modells.

\subsection{Modelle in der quantitativen Politikfolgenabschätzung - ein Überblick}

Die computergestützte quantitative Agrarsektormodellierung wurde in Deutschland in den 70er-Jahren im Rahmen des DFG-Schwerpunktprogramms „Konkurrenzvergleich landwirtschaftlicher Standorte“ aufgenommen. Aufbauend auf den Vorarbeiten von EARL O HeADy (Ames/Iowa, 1950/60) wurde unter Leitung von Professor Henrichsmeyer damals das interregionale Gleichgewichtsmodell QUISS entwickelt, das die landwirtschaftliche Produktion nach Regionen und Betriebsgruppen differenziert abbildet (HENRICHSMEYER, 1972). Es stellt einen wichtigen Vorläufer für die Modellsysteme SPEL, RAUMIS und CAPRI dar. ${ }^{1}$

Ihre Wurzeln hat die Modellierung des landwirtschaftlichen Sektors in den USA, wo u. a. im $\mathrm{CARD}^{2}$ interregionale Gleichgewichtsmodelle entwickelt wurden. Diese Modelle haben dann Ende der 70er-Jahre stark an Bedeutung eingebüßt, weil

- $\quad$ sie für die vorhandenen Rechenkapazitäten zu groß und wenig nutzerfreundlich waren und

- die Abbildung der Ist-Situation häufig über Einschränkungen des Lösungsbereiches erreicht wurde, was die Prognosegüte im Hinblick auf Politikalternativen einschränkte und wodurch die Ergebnisse dem Bedarf politischer Entscheidungsträger häufig nicht entsprachen (KLEINHANSS, 2000).

Die Anforderungen an die modellgestützte Politikanalyse änderten sich grundlegend mit der '92er-Reform der Gemeinsamen Agrarpolitik. Einerseits handelte es sich bei der

\footnotetext{
1 Übersichtsartikel auf folgender Internet-Seite: www.agp.uni-bonn.de/agpo/rsrch/quiss_d.htm.

2 Centre for Agricultural and Rural Development, Iowa State University, Iowa/USA.
} 
vorzunehmenden Entkopplung von Preis- und Einkommenspolitik um einen Strukturbruch, so dass die Wirkung von Politikänderungen durch Trendextrapolation oder ökonometrische Modelle nicht mehr hinreichend genau prognostiziert werden konnte. Andererseits setzten die Ausgestaltung und der Umfang der Transferzahlungen häufig bei regionalen/betriebsstrukturellen Merkmalen an, die wiederum betriebliche Anpassungen induzieren. Dies machte es notwendig, die Modellierung, die bisher auf die Sektorebene fokussierte, bis auf die Entscheidungsebene der Betriebe voranzutreiben (KLEINHANSS, 2000, S. 1-2).

Modellrechnungen auf Grundlage der Testbetriebe wurden zwar in der EU-Kommission und auch im BML durchgeführt. Mittels der hausintern durchgeführten Projektionen, ohne Berücksichtigung betrieblicher Anpassungen, konnten aber weder Einkommens- noch Angebotseffekte hinreichend abgeschätzt werden.

Spätestens seit diesem Zeitpunkt verstärkte sich in Brüssel und Bonn der Wunsch nach einer modellgestützten Politikberatung, und zwar nicht nur im Sinne der Folgenabschätzung beschlossener Maßnahmen, sondern auch im Vorfeld politischer Maßnahmen. Seitens des Bundesministeriums besteht seitdem Interesse an quantitativer Folgenabschätzung sowohl auf Sektor-, als auch auf Regions- und Betriebsebene. ${ }^{3}$

Die im Bereich Agrarökonomie der Bundesforschungsanstalt für Landwirtschaft (FAL) Mitte der 90er-Jahre verfügbaren Modelle trugen diesem Bedarf ebenfalls nur teilweise Rechnung. Damals waren ein partielles Gleichgewichtsmodell (GAPsi) und ein auf repräsentative Betriebe ausgerichtetes Betriebsmodell (BEMO) verfügbar. 1995 wurde RAUMIS vom Institut für Agrarpolitik, Marktforschung und Wirtschaftssoziologie der Universität Bonn (IAP) übernommen und gemeinsam weiterentwickelt (HENRICHSMEYER et al., 1996). Neuentwicklungen entsprachen dem deutlich gewordenen Bedarf nach Betriebsmodellen. Ab 1996 wurden FARMIS (JACOBS, 1998; SCHLEEF, 1999) auf der Grundlage von RAUMIS und ab 1997 TIPI-CAL (Technology Impact and Policy Impact Calculations) auf der Grundlage von FLIPSIM ${ }^{4}$ (Texas AFPC ${ }^{5}$ ) entwickelt. Bei FARMIS handelt es sich um ein Optimierungsmodell für Betriebsgruppen zur sektorkonsistenten Abbildung der deutschen Landwirtschaft, während TIPI-CAL ein dynamisches Simulationsmodell für typische Betriebe im weltweiten Vergleich auf Basis eigener Datenerhebungen zur Bil-

3 Diesem Bedarf konnte durch die nun erzielten Fortschritte in der Computertechnik mit vertretbarem Aufwand auch durch deutsche Forschergruppen entsprochen werden.

$4 \quad$ FLIPSIM ist ein zehnjähriges, einzelbetriebliches, dynamisches und stochastisches Simulationsmodell, welches flexibel konzipiert wurde, um aktuelle und zukünftige Politikvarianten für verschieden Betriebstypen abzubilden. Eine detaillierte Modellbeschreibung erfolgt bei RICHARDSON and NiXON (1986).

5 Agricultural and Food Policy Center der Texas A\&M University (AFPC). 
dung typischer Betriebe ist (vgl. hierzu Kapitel 3.2). 1999 ist das Institut für Marktanalyse und Agrarhandelspolitik dem GTAP (Global Trade Analysis Project) Konsortium beigetreten. Dieses Modellsystem bildet ganze Volkswirtschaften im Rahmen des Allgemeinen Gleichgewichtsansatzes ab, wobei diese miteinander durch bilateralen Außenhandel verknüpft sind und der Agrarsektor mit größerer Detailgenauigkeit als der Industrie- und Dienstleistungssektor berücksichtigt wird.

Die Bündelung von Informationen über Analysen auf der Grundlage verschiedener Betrachtungsebenen verspricht den Informationsgehalt der Analysen insgesamt zu verbessern. Daher wird im Rahmen von Modellverbünden versucht, Modellergebnisse auf der Grundlage unterschiedlicher Betrachtungsebenen miteinander zu verknüpfen. Die Verknüpfung kann dabei über modellendogene Hochrechnungen der Ergebnisse der unteren Betrachtungsebene auf die nächsthöhere Betrachtungsebene oder über einen wechselseitigen Informationsaustausch ohne modellendogene Verkettung erfolgen (GÖRTZ, 1998, S. 9; HENRICHSMEYER und KREINS, 1992; JACOBS, 1998; KLEINHANSS et al., 1998, 2001, 2002; SALAMON et al., 2002; ZEDDIES, 2003). Dieses Verfahren wurde seit Mitte der 90er-Jahre in der FAL vorangetrieben. Sektormodellergebnisse werden durch betriebliche Informationen oder Anpassungsreaktionen, die von Betriebsmodellen prognostiziert wurden, ergänzt. In einem Modellverbund werden auf verschiedene Bezugsysteme ausgerichtete Modelle eingesetzt, um die Komplexität agrarpolitischer Maßnahmen zu erfassen. Sie sollen dem Bedarf politischer Entscheidungsträger an differenzierten Betrachtungen nach Markt-, Sektor-, Regions-, Betriebs- und ggf. Verfahrensebene Rechnung tragen (BERTELSMEIER et al., 2003). Anpassungsreaktionen, die sich in höher aggregierten Modellen nicht oder nur auf Basis einfacher Annahmen abbilden lassen, werden in einzelbetrieblichen Modellen detailliert untersucht. Die Modelle können unter Umständen nach Rückkopplung mit den Landwirten modifiziert werden. ${ }^{6}$ Eine Darstellung über die Verknüpfung dieser Modelle im Rahmen des FALModellverbunds befindet sich im Anhang in Abbildung A3.1.

\subsection{Entwicklungsstand modellgestützter Analysen in Deutschland und Europa}

Die Folgen der in der Halbzeitbewertung der Agenda 2000 vorgeschlagenen Politikreform waren Gegenstand einer Vielzahl modellgestützter Analysen. Sie versuchten die möglichen Auswirkungen der zukünftigen gemeinsamen Agrarpolitik auf verschiedene Zielgruppen (Verbraucher, Erzeuger, Konsumenten oder Steuerzahler) oder Regionen abzu-

6 Im Rahmen bisher durchgeführter Politikfolgenabschätzungen wurden die Betriebsmodelle BEMO, FARMIS und TIPI-CAL komplementär zu Regions- und Marktmodellen eingesetzt. Auf Betriebsebene prognostizierte Anpassungsreaktionen werden soweit wie möglich von den auf höherem Aggregationsniveau arbeitenden Modellen übernommen. 
schätzen. Am Beispiel der Diskussion um die Reform des Milchmarktes kann die Breite der verwendeten Ansätze besonders gut verdeutlicht werden.

Tabelle 3.1 stellt eine Übersicht der im europäischen Raum zur Verfügung stehenden und verwendeten Modellsysteme zur quantitativen Analyse dar. ${ }^{7}$ Die überwiegende Zahl an Regionalmodellen verdeutlicht, dass im Vordergrund der Auswertungen noch immer die verhältnismäßig hochaggregierte Ebene des Sektors steht. Nur wenige Studien beziehen die Betriebsebene mit in die Betrachtung ein.

Tabelle 3.1: Hauptgruppen von Produktions- und Betriebsmodellen für die Politikanalyse

\begin{tabular}{lllll}
\hline Methode & $\begin{array}{l}\text { Lineare } \\
\text { Programmierung }\end{array}$ & $\begin{array}{l}\text { LP und ökono- } \\
\text { metrische } \\
\text { Schätzungen }\end{array}$ & PMP & Simulation \\
Ebene & BEMO, INEA & & FARMIS & TIPI-CAL \\
\hline Betrieb & & INRA-WAU & RAUMIS, CAPRI, & \\
Region & & $\begin{array}{l}\text { INEA, INRA } \\
\text { (AROPAJ/Jayet ) 1) }\end{array}$ & $\begin{array}{l}\text { (Paris-Howitt-Arfini) } \\
\text { (AROPA }\end{array}$ \\
\hline
\end{tabular}

1) Analysen zur Milchmarktreform im Rahmen der Agenda 2000 für mehrere EU-Länder im Auftrag der EU-Kommission durchgeführt - keine Veröffentlichung.

Quelle: Eigene Darstellung (2004).

Fast alle verwendeten Ansätze sind normative Analysen, die auf der mikroökonomischen Theorie fußen. ${ }^{8}$ Eine Ausnahme stellt die irische Studie dar. Hierbei handelt es sich um eine positive Analyse, die auf Umfrageergebnissen von 490 spezialisierten Milchviehbetrieben (siehe Tabelle 3.2) in Irland aus dem Jahr 1999 basiert (HENNESSY et al., 2000: TEAGASC). ${ }^{9}$ In dieser Studie werden Informationen zur Faktorverfügbarkeit und zu intermediären Inputs ergänzt mit eher qualitativen Angaben, wie z. B. Informationen über das Anpassungsverhalten der Landwirte, Pläne für zukünftige Investitionen und Stabilität

Als andere Auflistung quantitativer Modellansätze ist die von VAN TONGEREN und VAN MEUL (1999) zu nennen: „Review of applied models of international trade in agriculture and related resource and environmental modelling“.

8 Die mikroökonomische Theorie dient insbesondere im neoklassischen Theoriegebäude (neoklassische Theorie des Unternehmens) der Ableitung von Branchenangebotsfunktionen, die gemeinsam mit der Marktnachfrage unter Annahme vollkommener Konkurrenz den Marktpreis bestimmen (HENRICHSMEYER, 1991).

9 Irish Agriculture and Food Development Authority (Teagasc), Dublin/Irland. 
des Milchbetriebszweiges. Das Produktionspotenzial in 2008 wird aus den geschätzten Kuhbeständen und möglichen Effizienzgewinnen hergeleitet. Die berechneten Einkommenseffekte setzen die Verwirklichung des geschätzten Produktionspotenzials voraus. Zusätzlich werden mögliche Investitionsentscheidungen und daraus resultierende Einkommenseffekte analysiert. Die Ergebnisse für das Jahr 2008 reflektieren sowohl kurzfristige als auch langfristige Anpassungsreaktionen.

Alle anderen Studien basieren auf quantitativen Analysen mit unterschiedlichen Modellansätzen (LIPS et al., $2002^{10}$; JANSSON, 2002 ${ }^{11}$ ). Bei einigen Untersuchungen sind verschiedene Ansätze kombiniert, um der Komplexität agrarpolitischer Maßnahmen auf den unterschiedlichen Betrachtungsebenen gerecht zu werden (HELMING et al., 2002; VAN TONGEREN, 2002 ${ }^{12}$; COMMISSION OF THE EuROPEAN COMMUnities, $2002^{13}$; Kleinhanss et al., $2002^{14}$; Colman (Ed.), $2002^{15}$; ARFINI et al., $2002^{16}$; siehe Tabelle 3.2). Die verwendeten Modelle unterscheiden sich jedoch deutlich in der Aufgabenstellung und der Methode.

Sie umfassen

- $\quad$ allgemeine Gleichgewichtsmodelle, die ganze Volkswirtschaften abbilden (ETH, LEI),

- partielle Gleichgewichtsmodelle, die die wichtigsten Märkte für Agrarprodukte abdecken (FAL, SLI) oder speziell auf den Milchmarkt fokussieren (FAL, INRA-WAU),

- ökonometrische Angebotsmodelle (INRA-WAU),

- verschiedene Typen von (regionalen) Programmierungsmodellen (LEI, FAL, DEFRA, SLI)

- $\quad$ und expertenbasierte Simulationsmodelle (FAL).

Im Folgenden werden diese Modelle im Hinblick auf ihre Aussagekraft und ihre Eignung für die Fragestellung dieser Arbeit beleuchtet. Einen Überblick über die Modelleigenschaften und die zugrunde liegenden Szenarien und Ergebnisse geben die Anhangstabellen A3.1 und A3.2. Die folgenden Tabellen 3.2 und 3.3 zeigen einen kurzen Auszug daraus.

10 Swiss Federal Institute of Technology Zurich (ETH), Department of Agricultural Economics, Zurich/Switzerland.

11 Swedish Institute for Food and Agricultural Economics (SLI), Lund/Sweden.

12 The Agricultural Economic Research Centre (LEI), The Hague/Netherlands.

13 Studie Institute National de la Recherche Agronomique (INRA), Paris/Frankreich und Wageningen University (WAU), Department of Agricultural Economics, Wageningen/Niederlande. ländliche Räume und Institut für Marktanalyse und Agrarhandelspolitik, Braunschweig/Deutschland. 


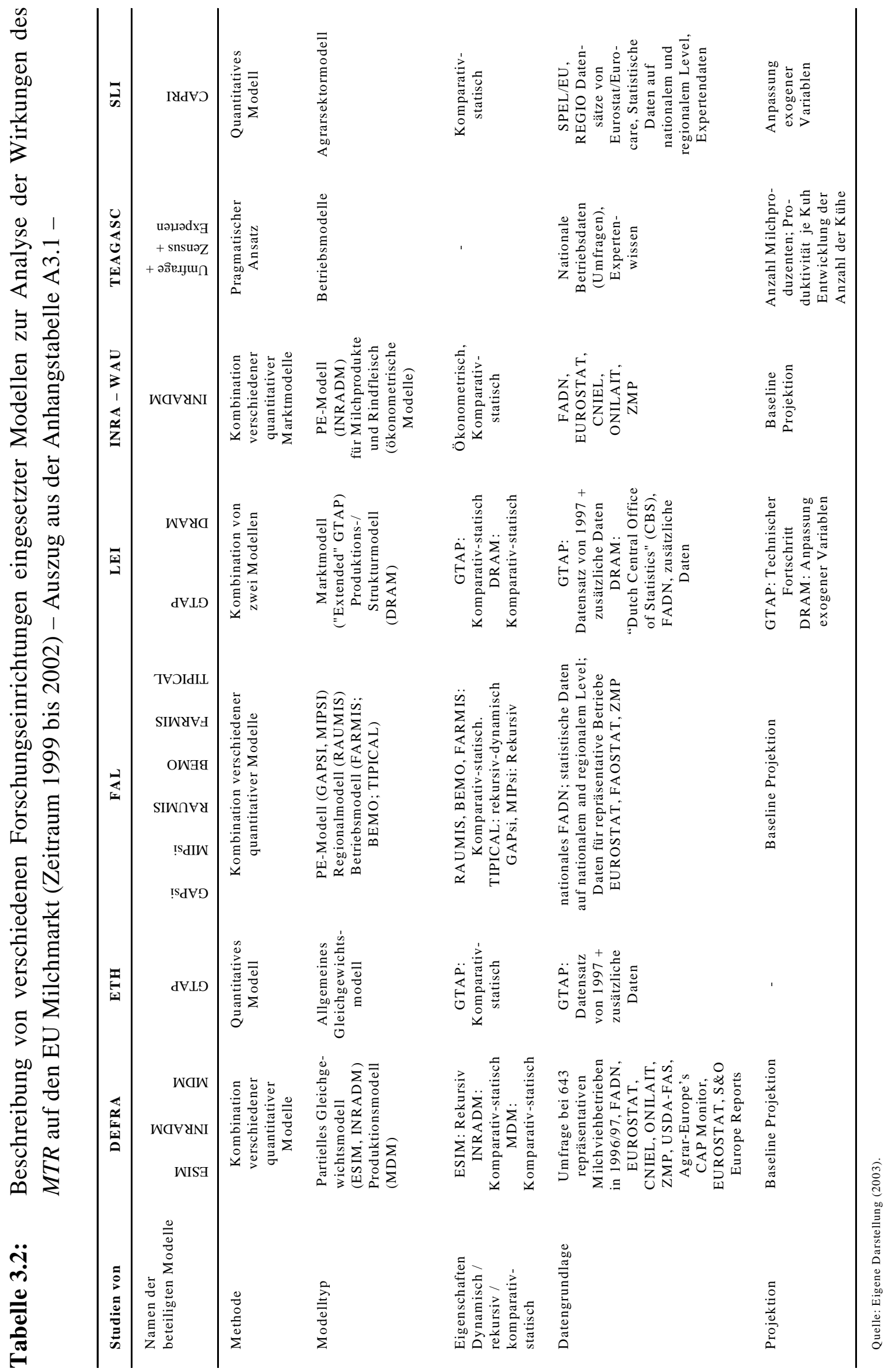


ฮั

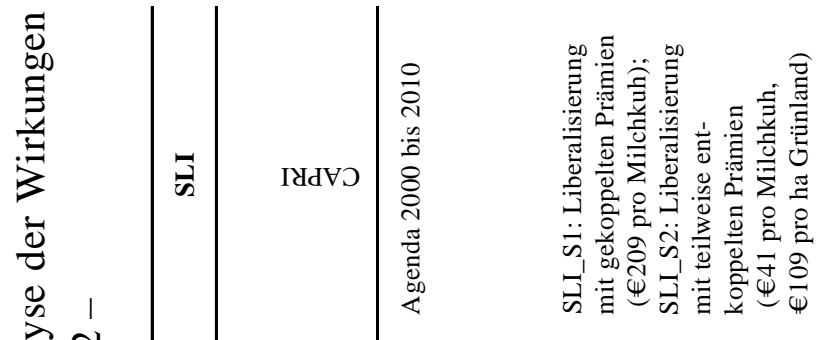

宽 ขั

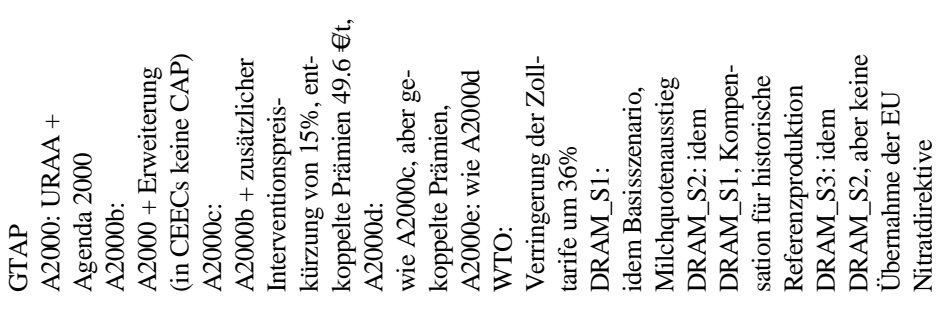

dVLO

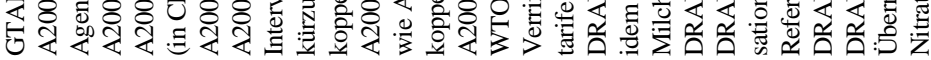
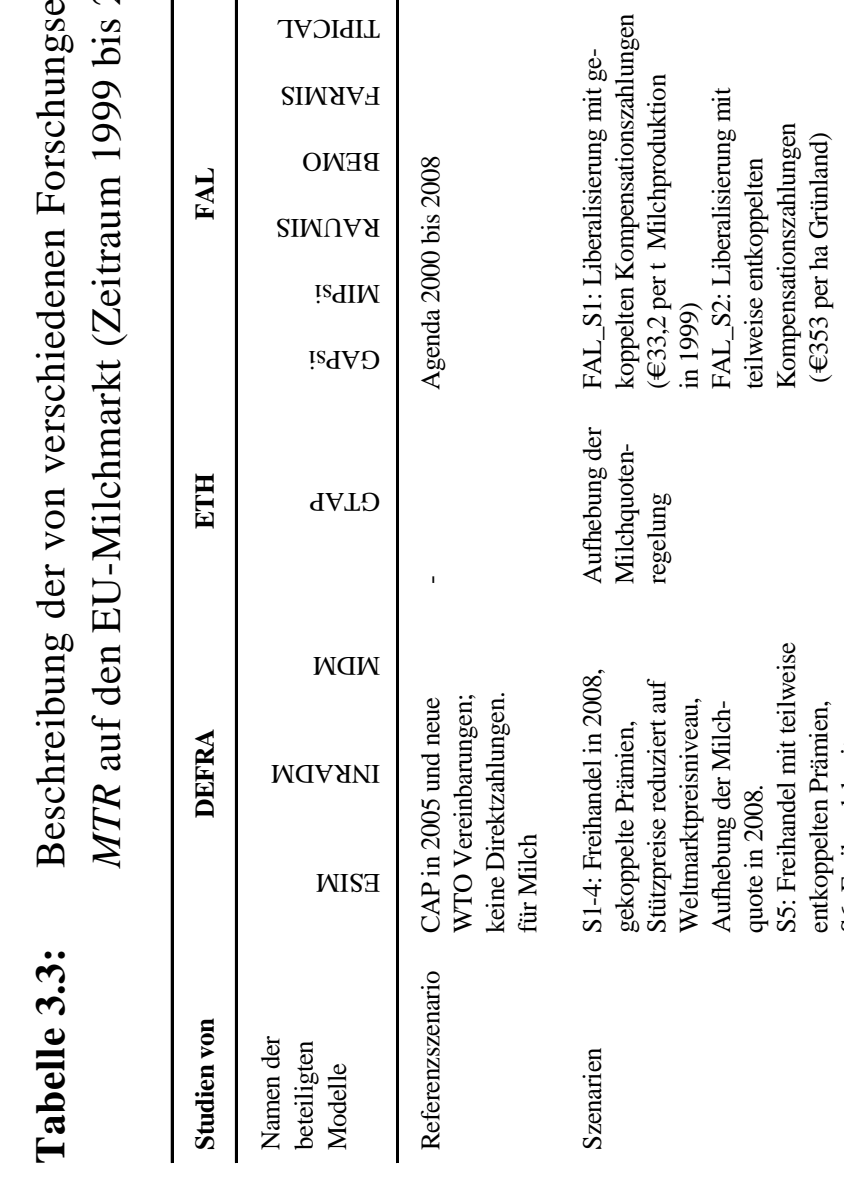

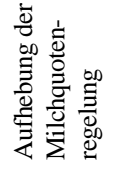

an
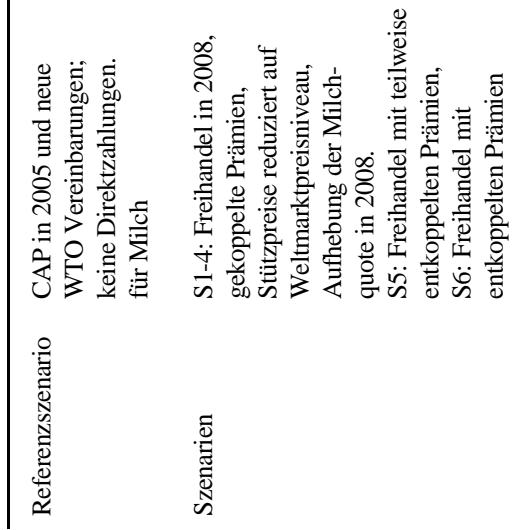


\subsubsection{Allgemeine Gleichgewichtsmodelle}

Allgemeine Gleichgewichtsmodelle ermöglichen Analysen, die die gesamte Volkswirtschaft eines Landes oder mehrerer Länder einbeziehen. In den beiden Studien von ETH und LEI, die auf allgemeinen Gleichgewichtsmodellen basieren, wird das GTAP (Global Trade Analysis Project) Modell (HERTEL, 1997) verwendet (siehe Tabelle 3.2). GTAP ist ein komparativ-statisches, multi-regionales und multi-sektorales Modell mit einer Datenbank, die bisher 66 Regionen und 57 Sektoren vom primären Sektor bis hin zu verarbeitenden Sektoren umfasst. Die Ergebnisse können je nach Regionen oder Produktgruppen in Abhängigkeit von der Fragestellung aggregiert werden. Die Abbildung des Handels basiert auf bilateralen Handelsmatrizen (Armington-Ansatz). Politische Interventionen sind in Form von Preiskorridoren, Einkommenstransfers und Direktzahlungen erfasst. Generell sind quantitative Maßnahmen als Zolläquivalente implementiert. In beiden Studien sind die Milchquoten als quantitative Restriktion bei der Rohmilchproduktion mittels eines komplementären Ansatzes programmiert. Unterschiede treten bei der Behandlung von Quotenrenten und bei der Implementierung weiterer EU-Marktpolitiken auf, wie beispielsweise Interventionspreise und Exportrestriktionen. Einschränkungen hinsichtlich der Vergleichbarkeit der Ergebnisse treten im Bereich der jeweils gewählten regionalen Abdeckung (ETH: EU-Mitgliedstaaten, LEI: EU-15 aggregiert) und der sektoralen Breite auf. In der LEI-Studie werden GTAP-Ergebnisse ergänzt mittels betrieblicher Simulationsergebnisse aus dem Dutch Regional Agricultural Model (DRAM).

\subsubsection{Partielle Gleichgewichtsmodelle}

Außer den allgemeinen Gleichgewichtsmodellen wurden auch partielle Gleichgewichtsmodelle zur Abschätzung der Auswirkungen der Milchmarktreform angewandt. Im Falle der Milchquotenanalyse kamen die in Tabelle 3.2 erwähnten Modelle in der Regel in Verbindung mit Produktions- und/oder Betriebsmodellen zum Einsatz (FAL, SLI). Die Produktabgrenzung der partiellen Gleichgewichtsmodelle ist unterschiedlich. Einige der partiellen Gleichgewichtsmodelle (z. B. GAPsi) decken den gesamten landwirtschaftlichen Sektor ab oder erfassen die wichtigsten Produkte der EU (ESIM, GAPsi), andere beziehen beispielsweise nur Milch und die wichtigsten Milchprodukte (MIPsi) sowie das Koppelprodukt Rindfleisch mit ein (INRADM). Ältere Versionen von ESIM waren aggregiert auf EU-Ebene, inzwischen ist ESIM aber nach EU-Ländern differenziert. ${ }^{17}$ Die Modelle unterscheiden sich hinsichtlich der Produktbreite, der dabei berücksichtigten Kreuzpreiseffekte und der jeweils abgebildeten Politiken bzw. Politikinstrumente. Außerdem differieren sie

17 In ROW („Rest of the World“) werden diese sonstigen für die abgebildeten Produktmärkte relevanten Länder zusammengefasst. 
auch im gewählten Basisjahr und in der verwendeten Datenbasis. Die Datengrundlage besteht maßgeblich aus Daten von EUROSTAT, die durch zusätzliche Quellen ${ }^{18}$ ergänzt werden (z. B. von der ZMP und nationalen Statistiken). Fast allen Studien (INRA-WAU, FAL, SLI) ist die Einbeziehung mehrerer Betrachtungsebenen von der Milcherzeugung, Milchverarbeitung bis hin zur Konsumentennachfrage nach Milchprodukten gemeinsam.

Die Studie von INRA-WAU verwendet ein partielles Gleichgewichtsmodell INRADM (INRA Dairy Model), welches die Marktreaktion für die gesamte vertikale Kette, vom Milchangebot über die Milchverarbeitung bis zum Endprodukt und zur Nachfrage nach Milchprodukten abdeckt. Das Verarbeitungs- und Nachfragemodell teilt das gesamte Milchangebot in Fett- und Proteinkomponenten auf. Die Verarbeitungstechnologie fließt in die Modellierung mit ein. Das Modell berücksichtigt elf Milchprodukte. Neben den EUMitgliedsländern (EU-15) werden vier zusätzliche Regionen und Importe aus dem Aggregat „Rest of the World“ modelliert. Die jährlichen Verschiebungen der Nachfragefunktionen verkörpern die dynamische Komponente.

In der FAL-Studie werden zwei verschiedene partielle Gleichgewichtsmodelle angewendet. Im Modell GAPsi (Gemeinsame Agrarpolitik simuliert; ein Multiprodukt-, multi- regionales Modell) werden Gleichgewichtspreise unter Berücksichtigung von Kreuzeffekten berechnet. Die applizierte regionale Aggregation schließt Daten der einzelnen EUMitgliedstaaten und das Aggregat „Rest of the world“ mit ein. Das Standardmodell umfasst die Produkte Getreide, Ölsaaten, Hülsenfrüchte, Kartoffeln und Zucker (-rüben) sowie Milch, Rindfleisch, Schaffleisch, Schweinefleisch, Geflügelfleisch und Eier. Mischfuttermittel (Futtergetreide, eiweiß- und stärkehaltige Komponenten) fließen als Input mit ein.

Außer GAPsi wird in der FAL-Studie ein zweites partielles Gleichgewichtsmodell verwendet, das Modell MIPsi (Milchmarktpolitik simuliert). MIPsi ist spezialisiert auf die Simulation der Effekte unterschiedlicher EU-Milchmarktpolitiken. Die regionale Aggregation ist dieselbe wie bei GAPsi, die Produktpalette unterscheidet sich jedoch deutlich. Auf der Produktionsseite (MIPsi) befindet sich als einziges Produkt Rohmilch, welches in fünf Milchprodukte verarbeitet wird (Frischmilch, Butter, Käse, Milchpulver, andere Produkte). Jedes Produkt besteht aus einem definierten Set preisabhängiger Mengen an Milchfett, Eiweiß und einem Rest aller weiteren Komponenten. Aufgrund der differenzierten Abbildung der Milchprodukte können sowohl Interventionspreise von Butter und Magermilch-

18

In den älteren Modellen wurden in der Regel Preiselastizitäten aus verschiedenen Quellen (z. T. Schätzungen) zusammengesucht und im Hinblick auf die Erfüllung der Konsistenzbedingungen kalibriert. Neuerdings liegen geschätzte Elastizitäten vor. Im EDIM - Projekt (INRA) wurden Schätzungen von JoNGENEEL (2005) und TONINI (2007) durchgeführt und in das Modell einbezogen (http://edim. vitamib.com, http://edim.vitamib.com/publications/reportsD0603). Im Modell erfolgt ebenfalls eine Kalibrierung der Elastizitäten. Für die Schätzungen kommen zunehmend Entropieansätze zur Anwendung. 
pulver als auch WTO-Restriktionen, die sich auf einzelne Produkte beziehen, im Modell abgebildet werden.

ESIM (European Simulation Modelling Framework) ist ein komparativ-statisches, multiregionales, rekursives, partielles Gleichgewichtsmodell, welches Handels- und budgetäre Ströme abbildet und auf linearen Angebots- und Nachfragefunktionen basiert (LILLARD, 1995). Die osteuropäischen Länder (CEEC) werden einzeln modelliert, die EU und das Aggregat „Rest of the world“ werden jeweils als eine Region behandelt. Es handelt sich um ein „multi-commodity“ Modell, in dem der Milchsektor in vier Produktbereiche differenziert wird: Rohmilch, Butter, Magermilchpulver und Käse. Die Nachfrage nach Arbeit, Kapital und nicht-landwirtschaftlichen, intermediären Inputs werden im Modell berücksichtigt.

All diese Modelle bilden den Milchsektor nur auf einem sehr hohen Aggregationsniveau ab, in der Regel nur mit wenigen Elastizitätskoeffizienten pro Land. Eine prozessanalytische Abbildung des betrieblichen Anpassungsverhaltens ist nicht möglich. In der Produktionsfunktion dieser Modelle wird nur ein Teil der potenziell möglichen betrieblichen Anpassungsreaktionen (in der Regel nur über technischen Fortschritt) berücksichtigt. Deshalb stellt sich hier insbesondere die Frage, wie durch Kombination mit anderen Modellen und zusätzlicher Datenerhebung die Aussagekraft der Ergebnisse erhöht werden kann. Im Folgenden wird detaillierter auf Stellenwert und Potenziale der Regionshof- und Betriebsmodelle im Rahmen der Auswertungen zur Agenda 2000 eingegangen.

\subsubsection{Regionshof- und Betriebsgruppenmodelle}

Die in den Tabellen 3.2 und 3.4 genannten Regionshof- und Betriebsgruppenmodelle (Prozessanalysemodelle) lassen sich unterscheiden in Programmierungsmodelle (z. B. RAUMIS, FARMIS, CAPRI, DRAM) und ein expertenbasiertes Simulationsmodell (TIPICAL). Die Betriebsgruppenmodelle verwenden als Datengrundlage in der Regel FADNDaten. Die erwähnten Betriebsmodelle sind ihrerseits meistens nur auf die nationale Ebene ausgerichtet. Das nationale Testbetriebsnetz ist in den meisten Fällen die Datenbasis für einige Betriebsmodelle (DEFRA, FAL, LEI). Vereinzelt werden zusätzlich Umfragen durchgeführt, um ergänzende Informationen zu erhalten. Ferner finden die Datensätze SPEL und REGIO (SLI, FAL) ${ }^{19}$ Verwendung. Die meisten Betriebsmodelle sind als ma-

19 Nähere Informationen zu diesen Datensätzen sind in Kapitel 4 dieser Arbeit oder unter folgenden Internetadressen nachzulesen:

www.agp.uni-bonn.de/agpo/rsrch/spel/spel-a_d.htm (SPEL)

www.eu-datashop.de/datenba/DE/allgem/reg_leit.htm,

www.iue.it/LIB/Eresources/E-data/Descriptions/regio.shtml (REGIO). 
thematische Programmierungsmodelle (MP-Modelle) mit einer hohen regionalen Differenzierung und einer breiten Abbildung unterschiedlicher Produktionsaktivitäten konzipiert.

Im Folgenden werden die Analyseschwerpunkte der angewendeten Prozessanalysemodelle herausgestellt, die sich aus der unterschiedlichen Kombination von Modellansatz und Datenbasis (siehe Tabelle 3.2) ergeben.

Im Modell CAPRI (Common Agricultural Policy Reform Impact Analysis) wird die EU in 200 Regionen untergliedert (weitestgehend im Einklang mit NUTS 2 Ebene) und die europäische landwirtschaftliche Erzeugung einschließlich der Vorleistungen abgedeckt. Das Modell besteht aus einem Angebots- und Marktmodul. Das Angebotsmodul wird abgebildet durch individuelle, nichtlineare Programmierungsmodelle für jede Region, die sich dann jeweils als ein regional aggregierter Betrieb (Regionshof) darstellt. Das Marktmodul kann als ein „multi commodity“ Marktmodell betrachtet werden, das die aggregierten Angebotsmengen aus den Regionalmodellen verwendet. Die Ermittlung des Marktgleichgewichtes von Angebot und Nachfrage erfolgt durch iterativen Einsatz des Angebots- und des Marktmoduls. Um mögliche Anpassungsreaktionen im Falle einer Änderung der Milchmarktordnung abzubilden, werden Schätzungen von Milchquotenrenten exogen vorgegeben.

RAUMIS (Regionalisiertes Agrar- und Umweltinformationssystem) ist ein Instrument zur Abbildung und Analyse des gesamtdeutschen Agrarsektors in tiefer regionaler Untergliederung. Das Modell ermöglicht Folgenabschätzungen für alternative agrar- und umweltpolitische Rahmenbedingungen. Mit RAUMIS können zukünftige Entwicklungen von Produktion, Faktoreinsatz und Wertschöpfung sowie Umweltwirkungen abgeschätzt werden. Kernstück von RAUMIS ist ein regionalisiertes, nichtlineares Programmierungsmodell.

Das deutsche Wirtschaftgebiet wird in 334 Modellkreise unterteilt. Jeder Modellkreis wird als landwirtschaftlicher Betrieb (Regionshof) betrachtet. Jeder Regionshof ist charakterisiert durch eine bestimmte Ausstattung an Primärfaktoren und spezifischen Produktionsfunktionen. Die regionale Abbildung des landwirtschaftlichen Sektors erfolgt in Konsistenz zur landwirtschaftlichen Gesamtrechnung. Die landwirtschaftliche Produktion wird prozessanalytisch nach einzelnen Verfahren differenziert und angebotsorientiert abgebildet. Wechselbeziehungen zwischen den Verfahren werden berücksichtigt. RAUMIS besteht aus einer Monitoringkomponente, die sich besonders für Ex-post-Analysen eignet, und einer Prognosekomponente für die Politikfolgenabschätzung.

Für die Politikfolgenanalyse wird versucht, die Anpassungsreaktionen auf veränderte Rahmenbedingungen über einen nichtlinearen Programmierungsansatz abzuschätzen. Die Zielfunktion maximiert die Nettowertschöpfung unter Berücksichtigung der Kosten für Arbeit. Das Ergebnis kann zum Agrarsektor aggregiert und nach Modellkreisen differen- 
ziert dargestellt werden. Als Datengrundlage fungieren landwirtschaftliche Fachstatistiken, KTBL-Daten, Daten der Landwirtschaftlichen Gesamtrechnung und Expertenwissen. Das Modell ist besonders geeignet, um sektorale Gleichgewichte oder budgetäre Begrenzungen abzubilden und gleichzeitig das Gleichgewicht zwischen Angebot und Nachfrage für Jungvieh zu gewährleisten. Im Rahmen der Studien zur Analyse einer Änderung in der Milchmarktpolitik sind diese Aspekte von besonderer Bedeutung für die Vorhersage der Rindfleischproduktion. RAUMIS wird in der FAL iterativ mit dem partiellen Gleichgewichtsmodell GAPsi verwendet, um Angebotsveränderungen im Marktmodell zu kalibrieren und konsistente Preisszenarien festzusetzen.

Das mathematische Programmierungsmodell DRAM (Dutch Regional Agricultural Model), das auf regional aggregierten Betrieben in den Niederlanden basiert, bildet produktions- und strukturelle Effekte, wie beispielsweise die regionale Veränderung der Milchproduktion, als auch technische Änderungen ab. Es differenziert 14 landwirtschaftliche Regionen in den Niederlanden und soll die landwirtschaftlichen Produktionsaktivitäten möglichst detailliert wiedergeben. Die Milchproduktion wird in neun verschiedenen Betriebsaktivitäten widergespiegelt. Die Gruppierung erfolgt nach ökonomischen und umweltrelevanten Kriterien: a) nach der Milchproduktion pro Kuh und b) nach dem Stickstoffeintrag pro Hektar Grünland.

Mit Hilfe des PMP Ansatzes (HowITT, 1995) ${ }^{20}$ werden die technischen Koeffizienten der unterschiedlichen Produktionsaktivitäten in Anlehnung an die Ausgangswerte im Basisjahr kalibriert. Grundannahme für das Verhalten im landwirtschaftlichen Sektor ist die Gewinnmaximierung. Die Inputs und Outputs der einzelnen Betriebe werden in DRAM auf Regionsebene aggregiert. Die Datengrundlage bilden im Wesentlichen Zensusdaten (Anbauverhältnisse und Tierzahlen, $\mathrm{CBS}^{21}$ ) und nationale FADN-Daten. ${ }^{22}$

Ein Vorteil von DRAM ist, dass es die landwirtschaftliche Produktion in der Ist-Situation sehr detailliert nach einzelnen Aktivitäten erfasst. Daher können Wechselbeziehungen zwischen den verschiedenen Aktivitäten berücksichtigt werden. In Abhängigkeit von der Szenariospezifikation können auch technologische Änderungen analysiert werden. Außerdem trägt dieses Modell dem Problem der Düngerverteilung und Düngerbilanz besonders Rechnung. Ein Nachteil liegt darin, dass die individuellen betrieblichen Aktivitäten zu

20 Das Hauptziel ist nicht die „optimale“ Produktionskombination zu ermitteln (normative LP Modelle), sondern ein möglichst realistisches Bild der Situation und der Verhaltensweisen der Landwirte zu simulieren (positive Verfahren).

21 Die Zensusdaten (Landwirtschaftszählung in den Niederlanden) werden vom Central Office of Statistics (CBS) erhoben.

22

Die Stichprobe aus dem nationalen FADN umfasst 1.000 Betriebe, die 95 \% der Produktion und $65 \%$ der Betriebe in den Niederlanden repräsentieren (HELLMING, 2002). 
Gruppen von Produktionsaktivitäten auf regionalem Niveau aggregiert werden, damit Modell und Berechnungsdauer handhabbar bleiben. Dies hat zur Folge, dass das Verhalten der Betriebsleiter und strukturelle Unterschiede zwischen den Betrieben nur vereinfacht abgebildet werden können. Betriebliches Wachstum oder Desinvestitionsstrategien als Folge von politischen Maßnahmen lassen sich modellendogen in all diesen Modellen kaum zutreffend vorhersagen.

Im Modell FARMIS (Farm Group Model for the German Agricultural Sector) sind homogene Betriebsgruppen nach bestimmten Schichtungskriterien wie Region, Betriebstyp und Betriebsgröße für Deutschland aggregiert. Kern dieses komparativ-statischen Modells, das in der FAL Anwendung findet, ist ein nach Betriebsgruppen differenziertes, nichtlineares Programmierungsmodell. Im Vergleich zum Testbetriebsnetz verbesserte Hochrechnungsfaktoren (siehe Dissertation JACOBS, 1998) ermöglichen die Hochrechnung der Ergebnisse auf Sektorebene sowohl für monetäre als auch für physische/technische Indikatoren. Die landwirtschaftliche Produktion wird prozessanalytisch nach einzelnen Verfahren differenziert und angebotsorientiert abgebildet. Wechselbeziehungen zwischen den Verfahren werden berücksichtigt. Wie im RAUMIS maximiert die Zielfunktion die Nettowertschöpfung unter Berücksichtigung der Kosten für Arbeit. Die Modellstruktur und die Definition der unterschiedlichen Aktivitäten sind nahezu dieselben. Beide Modelle werden in der FAL eingesetzt, um Auswirkungen von Politikänderungen auf regionaler Ebene und auf Betriebsebene abzuschätzen.

In FARMIS erfolgt eine Bildung von Betriebsgruppen, die nach den Schichtungskriterien Region (Bundesland), Betriebsform und Betriebsgröße (Standardbetriebseinkommen) abgegrenzt werden. Der Einsatz von Betriebsgruppen im Gegensatz zu Einzelbetrieben hat den Vorteil einer besseren Handhabbarkeit und reduziert den Einfluss von Datenfehlern und Datenlücken. Aufgrund der Aggregation von Einzelbetrieben zu Betriebsgruppen im FARMIS Modell sind bestimmte Effekte, wie beispielsweise die Produktionswirksamkeit einer Prämie, erst bei größeren Preissenkungen zu beobachten (KLEINHANSS et al., 2001). Die Datengrundlage von FARMIS bilden die jährlich erhobenen Buchführungsergebnisse der BMVEL-Testbetriebe, die insgesamt etwa 11.000 Betriebe mit je ca. 8.500 Variablen umfassen. Um (zum Teil witterungsbedingte) Schwankungen von Jahr zu Jahr zumindest teilweise zu unterbinden, werden Durchschnittswerte identischer Betriebe zweier aufeinanderfolgender Wirtschaftsjahre herangezogen. Ergänzt werden diese Daten durch technische Koeffizienten, die verschiedenen Publikationen des $\mathrm{KTBL}^{23}$ entnommen sind, sowie durch Experteneinschätzungen. Als wesentliches Merkmal von FARMIS ist hervorzuheben, dass die für die Betriebsgruppen ermittelten I-/O-Koeffizienten konsistent zum Buchführungsabschluss sind. Im Modell werden 27 pflanzliche und 15 tierische Hauptverfahren 
unterschieden. Die Kalibrierung des Modells erfolgt über den Ansatz der positiven quadratischen Programmierung. Die optimalen Produktionsumfänge ergeben sich durch eine Maximierung des Betriebseinkommens abzüglich der Opportunitätskosten für Arbeit sowie der Fremdzinsen unter Einhaltung der formulierten Nebenbedingungen. ${ }^{24}$

BEMO verwendet im Rahmen der Studien zur Änderung der Milchmarktpolitik als Datenbasis 4.800 Milchviehbetriebe, die im deutschen Testbetriebsnetz vorkommen. Verbunden mit einer leistungsfähigen Rechentechnik ermöglicht dies zum einen, betriebliche Entscheidungssituationen auf einzelbetrieblicher Ebene abzubilden und zum anderen die Ergebnisse mittels Hochrechnungsverfahren auf Sektorebene hochzurechnen. Die Modellgenerierung erfolgt mit Excel-Tabellen, die Optimierung mittels XA-Solver. ${ }^{25}$ Dieser Bottom-up-Ansatz erlaubt es, differenzierte Folgewirkungen auf repräsentativer Grundlage abzuschätzen und darüber hinaus Fragestellungen wie Milchquotenhandel sowie die differenzierte Ausgestaltung von Politikinstrumenten zu untersuchen. Da im Modell Sonderkulturen, Gartenbau, Schaf- und Geflügelhaltung bisher nicht abgebildet werden, müssen alle auf diese Betriebszweige ausgerichteten Betriebe ausgeschlossen werden. Formal ist das Modell für alle im Testbetriebsnetz vorhandenen ca. 8.500 Marktfrucht-, Futterbau-, Veredlungs- und Gemischtbetriebe anwendbar. Das Modell dient insbesondere dazu, den Milchquotenhandel zwischen den Betrieben abzubilden. Ferner werden kurz- und mittelfristige Anpassungsreaktionen abgeschätzt, wobei auch hier die Problematik einer unzureichenden modellendogenen Abbildung einzelbetrieblicher Investitions- und Desinvestitionsstrategien besteht. Deshalb wurde z. B. mangels betriebsspezifischer Informationen über Stallkapazitäten versucht, kurz- und langfristige Anpassungsspielräume auf Grundlage von Befragungen und Expertengespräche im Rahmen des Netzwerkes typischer Betriebe (IFCN-Konzept) einzugrenzen.

Die INEA-Programmierungsmodelle stellen die Heterogenität der italienischen Milchproduktion differenziert nach 25 Betriebstypen dar. Dieser Ansatz wurde gewählt, um die Verhaltensweisen der Landwirte bei Politikänderungen abzubilden. Der Ansatz soll die betriebliche Situation (Produktion, Einkommen) vor und nach der Maßnahme möglichst realitätsnah widerspiegeln. Gleichzeitig soll auch den jeweiligen Marktänderungen Rechnung getragen werden. Man verwendet den „Positive Mathematic Programming“ (PMP) Ansatz (HowITT, 1995), der Auswertungen auf Regions- und Sektorebene ermöglicht, auch wenn spezielle Buchführungsdaten nur begrenzt verfügbar sind. Die Integration des Outputs aus den ökonometrischen Teilmodellen ermöglicht detailliertere Abschätzungen

24 Eine detailliertere Darstellung zum Aufbau des Modellsystems FARMIS ist in Abbildung A3.2 im Anhang nachzulesen.

25

Die Optimierung mit XA-Solver ermöglicht eine geringere Rechenzeit bei Verwendung leistungsfähiger Prozessoren. 
der Auswirkungen auf regionaler Ebene durch das Gesamtsystem. Mit Hilfe des PMP Ansatzes werden ökonomische und strukturelle Informationen aus verschiedenen Datenbanken - z. B. FADN, SPEL - zur Abbildung von Anpassungsverhalten verwendet und Modelle konstruiert, die Kalibrierungs- und Prognoseelemente enthalten. Damit soll ein möglichst dynamisches Bild über die Effekte von Politikänderungen gegeben werden. Jedes neue Szenario führt zu einer Anpassung von Produktion und Verarbeitung. Dies erfolgt unter der Annahme von Gewinnmaximierung.

Bei den in der Studie des INRA verwendeten ökonometrischen Modelle werden Kostenfunktionen für alle EU-Mitgliedstaaten auf Basis der FADN-Daten geschätzt. Man erhält hier Informationen über Schattenpreise für Milchquoten durch Ableitung einer Milchschattenpreisfunktion auf Basis der Dualitätstheorie. Diese Information wird dann verwendet, um die Schattenpreisangebotsfunktion für Milch zu kalibrieren. Das Produktionsmodell INRADM, das in Zusammenarbeit mit der Universität Wageningen entwickelt wurde, basiert auf dualen kurzfristigen Gewinn- und Kostenfunktionen. Zusätzlich erfolgte eine Integration von Anpassungskomponenten für die Herdengröße und die bewirtschaftete Fläche. Ähnlich dem INEA Modell wird hierbei eine kurzfristige implizite Angebotsfunktion geschätzt mit dem Ziel, Milchangebotseffekte nach einer Änderung der Milchmarktordnung (Aufhebung der Milchquotenregelung) abzuschätzen. In dem Modell wird eine langfristige Kostenfunktion berechnet, um die Änderungen in der ökonomischen Überlebensfähigkeit unterschiedlicher Betriebsgrößenklassen abzuschätzen.

Das $\mathbf{M D M}^{26}$ (Manchester Dairy Model) ist ein einjähriges komparativ-statisches Milchangebotsmodell. Es wird im Rahmen von Studien zur Änderung der Milchmarktpolitik (vgl. Tabelle 3.2) verwendet, um die Milchpreisprojektion für das Vereinigte Königreich (UK) und Irland aus dem INRADM Modell in detailliertere Auswertungen speziell für den UKRohmilchproduktionssektor (Milchviehbetriebe) zu transferieren. Das Modell basiert auf Daten aus ca. 557 repräsentativen Milchviehbetrieben in UK. Ein zusätzliches Simulationsmodul bildet ökonomisches Anpassungsverhalten bei variierenden durchschnittlichen Milchpreisen $^{27}$ ab. Es unterstellt Gewinnmaximierung als einziges betriebliches Ziel. Mit einem Quotensystem können die repräsentativen Betriebe ihre Produktion nur ausweiten, wenn andere Betriebe ihre Produktion einschränken oder aus der Produktion aussteigen. MDM generiert Projektionen für die Milchproduktion in den einzelnen UK-Regionen. Das MDM teilt die Milchproduktion den verschiedenen Regionen und Betriebstypen auf Grundlage der relativen ökonomischen Effizienz der Betriebstypen zu. Die Kostenfunktio-

26

Entwickelt von COLMAN et al. (1998, 2001).

27 Jeder einzelne Referenzbetrieb hat einen eigenen individuellen Milchpreis. Dieser wird ermittelt mit Hilfe von beobachteten Differenzen zwischen betrieblichen und national durchschnittlichen Milchablieferungscharakteristiken, beeinflusst von der Ausgestaltung der Verträge, der jeweiligen Zusammensetzung der Milch, dem Standort und anderen betriebsspezifischen Faktoren (COLMAN, 2002). 
nen spiegeln verschiedene Milchviehbetriebsgrößen, Regionen und Leistungscharakteristika wie z. B. die Milchleistung wider. Die Version des Modells zum Zeitpunkt der Erstellung dieser Arbeit basiert auf Aufwendungen aus dem WJ 1999/2000 (nationales Testbetriebsnetz), die Kostenfunktionen ihrerseits spiegeln die technischen Effizienzparameter aus dem WJ 1996/97 wider. Das MDM fokussiert auf die Auswirkungen einer Änderung der Milchmarktpolitik für das Angebot an Milch in UK und auf die Anzahl von Milchviehbetrieben differenziert nach Bestandsgrößenklasse und Region.

TIPI-CAL (Technology Impact and Policy Impact Calculations) ist ein einzelbetriebliches, expertengestütztes, dynamisches Simulationsmodell zur Bewertung von Anpassungsstrategien typischer Modellbetriebe bei technologischen Änderungen und Politikänderungen. Im Rahmen der Auswertungen zur Milchmarktreform wird TIPI-CAL speziell zur Definition betrieblicher Anpassungsstrategien auf Basis von Expertenwissen verwendet. Das Modell wurde am Institut für Betriebswirtschaft an der Bundesforschungsanstalt für Landwirtschaft (FAL) in Braunschweig entwickelt. Das Modell TIPI-CAL wird von den internationalen Netzwerken IFCN (www.ifcnnetwork.org) und Agribenchmark (www.agribenchmark.org) genutzt. Das Ziel der Netzwerke ist es, eine weltweit harmonisierte Datenbasis für einzelbetriebliche Vergleiche und eine Wissensbasis aufzubauen, welche für Politikfolgenabschätzungen verwendet werden können. Der Modellinput besteht aus zwei Teilen. Im ersten Teil sind alle einzelbetrieblichen Daten und Konzepte für betriebliche Entwicklungsstrategien zusammengefasst. Der zweite Teil enthält Projektionen der Preise, Erträge und Inflationsraten für die Simulationsperiode. Derzeit können die Bereiche Milchproduktion, Mutterkuhhaltung, Rindermast, Ackerbau und Feldfutterbau abgebildet werden. Das Modell ist modular aufgebaut. Die bedeutendsten Produktionsstrukturen in den verschiedenen Ländern werden mittels sogenannter „typischer Betriebe“ dargestellt. Sie werden mit dem Expertenwissen von Landwirten, Beratern und Wissenschaftlern aufgebaut, das sich in detaillierten Daten und prozessspezifischer Differenzierung niederschlägt. Die enge Anbindung und Rückkopplung zur landwirtschaftlichen Praxis ermöglicht Plausibilitätsüberprüfungen der Ergebnisse. Insbesondere können produktionstechnisch bedingte Ursachen für Kostenunterschiede und betriebliches Anpassungsverhalten praxisnah erfasst und überprüft werden.

Einen Überblick über die berechneten Szenarien und Ergebnisse zur Folgenabschätzung von Milchquotenausstiegsszenarien liefern - wie oben erwähnt - die Anhangstabellen A3.2 sowie ein Auszug aus Tabelle 3.3 (Kapitel 3.2). In allen Studien wird eine schrittweise Aufhebung der Milchquotenregelung simuliert, dennoch differieren die Berechnungen zum Teil deutlich. Ein großer Unterschied zwischen den Studien besteht in der Projektionsperiode und in der Implementierung künftiger Politikänderungen wie der Agenda 2000. 
Die Produktions- und Betriebsmodelle ermöglichen insbesondere einen tieferen Einblick in die Effekte unterschiedlicher Direktzahlungssysteme. So werden in der FAL-Studie zwei verschiedene Systeme, Milch- und Grünlandprämien, berechnet. Dasselbe gilt auch für die Milchstudie mit dem CAPRI-Modell. Sie unterscheidet zwischen produktionsgebundenen und entkoppelten Direktzahlungen. Die DEFRA-Studie berechnet drei verschiedene Typen von gekoppelten und entkoppelten Direktzahlungen. Mit DRAM werden neben dem Quotenausstiegsszenario zwei weitere Szenarien, mit Milchprämien und Umweltrestriktionen, berechnet.

Neben den verschiedenen Kombinationen von Modellstruktur und Datengrundlage führen auch Variationen in den Annahmen und Szenarien zu unterschiedlichen Auswirkungen. Die meisten Studien reflektieren auch nationale Sichtweisen, die sich in der Szenariogestaltung und im Modelldesign widerspiegeln. Aufgrund unterschiedlicher politischer Rahmenbedingungen variieren beispielsweise die ermittelten Erzeugerpreisveränderungen bei einer Aufhebung der Milchquotenregelung von 12 bis 42 \%, die größeren Preiseffekte ergeben sich bei den produktionsgebundenen Prämien. Die Interaktion der im Tandem oder Verbund verwendeten Modelle führt zu weiterreichenden Erkenntnissen als es bei der separaten Herangehensweise möglich wäre. In den meisten Studien werden allgemeine und partielle Gleichgewichtsmodelle mit Produktions- und Betriebsmodellen kombiniert.

\subsection{Anforderungen an einen geeigneten Analyseansatz}

Im Hinblick auf die Fragestellung dieser Arbeit ist aus der Beschreibung der verschiedenen Modellansätze in Kapitel 3.2 folgende Feststellung zu ziehen:

Auf Markt- und Sektorebene liegen umfangreiche EU-weit vergleichende Analysen zur Politikfolgenabschätzung für den EU-Milchmarkt vor. Diese liefern u. a. Prognosen zu den Auswirkungen auf die nationalen Marktpreise und auf die Weltmarktpreise sowie auf die produzierten, konsumierten und gehandelten Mengen.

Darüber hinaus gibt es eine Reihe länderbezogener Studien. Die ermittelten Ergebnisse umfassen u. a. Abschätzungen der Entwicklung von Produktion, Faktoreinsatz, Wertschöpfung und Umweltwirkungen.

Bei der Analyse der Wirkungen von Politikänderungen im Bereich Milch kommen relativ selten betriebliche Modelle zum Einsatz. Insbesondere die Abschätzung von Einkommensänderungen und Anpassungsverhalten und deren Auswirkungen auf die Betriebseinkommen ist dem Modellüberblick zufolge nur sehr selten anzutreffen. Regionale Durchschnittsergebnisse sind für eine nach Betriebstypen und Produktionssystemen differenzierte Analyse häufig nicht ausreichend. Markt- und Regionalmodelle sind nicht in der Lage, 
verfahrenstechnische Besonderheiten so differenziert und detailliert abzubilden, wie Betriebsmodelle das ermöglichen (ARFINI, 2001, S. 14). Bei den Handlungskomponenten/Entscheidungsbeschränkungen fehlen oft nach Betriebstypen und Betriebsgrößenklassen differenzierte monetäre und technische Kenngrößen.

Da sich die Rahmenbedingungen für die Milchviehhaltung grundlegend verändern werden, besteht eine besondere Herausforderung darin, realitätsnahe Anpassungsreaktionen der Betriebe zu ermitteln und hierbei vor allem das Wachstum bzw. die Aufgabe der Milchviehhaltung in den Betrieben und Regionen realitätsnah abzubilden. In einigen Modellen wird versucht, dynamische Elemente einzubauen, z. B. durch die Integration endogenen technischen Fortschritts, d. h. durch die kontinuierliche Aktualisierung der Kapazität durch endogene Investitionen oder andere Merkmale, die es dem Modell ermöglichen, Entwicklungen im Zeitablauf zu simulieren (Burrell, 1995). Die Abbildung des betrieblichen Anpassungsverhaltens ist jedoch in allen hier vorgestellten Modellen begrenzt.

Je nach Betriebsmodell fällt die Abbildung der Entscheidungsvorgänge in Abhängigkeit von der verwendeten Modellstruktur und Datengrundlage sehr unterschiedlich aus. Aus den genannten Studien lassen sich folgende Defizite im Hinblick auf die Zielsetzung dieser Arbeit feststellen:

- Die meisten betrieblichen Analysen sind regional bzw. national begrenzt ${ }^{28}$, so dass die Vergleichbarkeit der Ergebnisse nur national gegeben ist.

- Teilweise basieren sie auf Fallstudien mit einer kleinen und nicht repräsentativen Anzahl von Betrieben. ${ }^{29}$

- Die meisten Modelle sind komparativ-statisch. Im Hinblick auf betriebliche Anpassungsreaktionen bei Politikänderungen wären dynamische Ansätze von Vorteil (vgl. KLEInHANSs, 2000). Diese würden die Darstellung einer partiellen, kurzfristigen Anpassung ermöglichen und eine Reihe zeitspezifischer Ergebnisse generieren, die sowohl die Größenänderungen der Anpassungsveränderungen aufzeigen als auch die Geschwindigkeit, mit der sie sich im Zeitablauf vollziehen.

- Die meisten Kombinationen von Modell und Datenbasis sind nicht in der Lage, die landwirtschaftliche Produktion prozessanalytisch, d. h. nach einzelnen Verfahren differenziert, abzubilden. ${ }^{30}$ Die Zuordnung von Investitionen und Gemeinkosten zu den einzelnen Verfahren bereiten meistens erhebliche Probleme. Die Berücksichtigung von Wechselbeziehungen zwischen den Verfahren wird dadurch erschwert.

MDM, BEMO, FARMIS, Mathematische Programmierungsmodelle der INEA.

29 Analysen vom TEAGASC, TIPICAL/IFCN.

30 MDM, BEMO, FARMIS, Mathematische Programmierungsmodelle der INEA. 
- Bei den Betriebsmodellen (z. B. bei LP-Ansätzen) ${ }^{31}$ besteht häufig die Schwierigkeit, betriebliche Wachstumsprozesse realitätsnah darzustellen.

- Eines der Hauptprobleme des Gesamtsystems bestehend aus Modell und zugehöriger Datenbasis ist eine geeignete, auf den Umfang des zu modellierenden Marktes oder der Regionen kompatible, nachhaltige ${ }^{32}$ und detaillierte betriebliche Datengrundlage. Diese steht für Modellsysteme, die nicht auf Testbetriebsdaten aufbauen, nicht zur Verfügung.

Aus den bisher diskutierten Modelleigenschaften und dem grundsätzlichen Problem einer ausreichend differenzierten Datenbasis (vgl. auch Studie von BRÜMMER, VON CRAMONTAubadel, Koesters und Meyer, 2002) für betriebliche Folgenabschätzungen lassen sich für die vorliegende Fragestellung folgende Anforderungen an einen Analyseansatz, der eine genauere Abbildung der einzelbetrieblichen Einkommens- und Angebotsänderungen ermöglichen soll, ableiten:

1. Die einzelbetriebliche Ebene sollte im Mittelpunkt stehen oder zumindest sollten die (betrieblichen) Datensätze nach verschiedenen Kriterien gruppierbar sein, damit Analysen möglich werden, die Variationen des Betriebs- und Regionstyps im jeweiligen Land berücksichtigen können.

2. Prozessspezifische Differenzierung. Der Modellansatz sollte die landwirtschaftliche Produktion prozessdifferenziert abbilden können. Dazu sind Datensätze erforderlich, die auf der Ebene von Produktionsverfahren oder zumindest in Form von Betriebszweigabrechnungen vorliegen, um Ungenauigkeiten bei der Kostenzuteilung zu den einzelnen Produktionszweigen zu vermeiden. Um diesem Problem zu entgegnen, besteht methodisch die Möglichkeit, die Analyse auf spezialisierte Betriebe zu begrenzen. $^{33}$ Je höher der Spezialisierungsgrad, umso höher ist die Genauigkeit der geschätzten oder kalkulierten Produktionskosten. Der variierende Anteil Gemischtbetriebe an der Gesamtproduktion kann dann jedoch nicht berücksichtigt werden.

31 Da bei den klassischen LP-Ansätzen das Produktionsprogramm vom innerbetrieblich fixierten Betriebsgrößenrahmen abhängt, weichen die Ergebnisse längerfristiger Abschätzungen betrieblicher Reaktionen häufig von denen in der Realität beobachteten Anpassungsreaktionen ab. Kurzfristige Abschätzungen mit marginalen Mengenänderungen können dafür realitätsnah dargestellt werden.

Die Möglichkeit nachhaltig Strukturen und Modelle bereitzustellen, mit denen auch nach Abschluss des Forschungsvorhabens schnell aktualisierte Ergebnisse produziert werden können (HEMME, 2000).

Der Spezialisierungsgrad eines Betriebes wird in der Europäischen Buchführungsstatistik in der Regel am Anteil des Standarddeckungsbeitrages des betrachteten Betriebszweiges am Gesamtdeckungsbeitrag des Betriebes gemessen (KOM-EG, 1990; AGRARBERICHT, 1992). Spezialisierte Milchviehbetriebe beispielsweise sind demnach solche, deren Standarddeckungsbeitrag aus der Milchproduktion einen Anteil von mindestens zwei Drittel am Gesamtstandarddeckungsbeitrag des Betriebes hat. 
3. Die Preise einerseits und die Mengenangaben andererseits sollten getrennt erhoben und ausgewiesen werden, um deren Auswirkungen auf die Produktionskosten isolieren zu können.

4. EU-weit harmonisiert. Der Aufwand für Zusammenstellung und Harmonisierung der Datenquellen verschiedener aufeinander abgestimmter Modellansätze sollte möglichst in Grenzen bleiben. Die Datensätze sollten allerdings EU-weit verfügbar und international kompatibel sein, d. h. einem einheitlichen Erhebungs- und Berechnungsmodus folgen, damit die Vergleichbarkeit zwischen den Ländern gegeben ist.

5. Repräsentativität. Die errechneten einzelbetrieblichen Auswirkungen sollten für eine typische Auswahl der Betriebe und Betriebssysteme einer Region gelten und damit die Grundgesamtheit der Betriebe gut repräsentieren, nicht nur hinsichtlich der regionalen Merkmale, sondern auch bezüglich der Vielfalt der Betriebstypen.

6. Aktualität und Prognosemöglichkeiten. Die Daten sollten aktuell sein. Sie sollten nicht nur für einen Zeitpunkt erhoben worden sein, sondern die Entwicklung einzelner Betriebe über einen längeren Zeitraum nachvollziehen lassen, um betriebliche Entwicklungen unter verschiedenen sich verändernden politischen und technologischen Rahmenbedingungen projizieren/modellieren zu können. Dafür ist ein dynamischer Modellansatz erforderlich, der die betriebliche Entwicklung über einen längeren Zeitraum aufzeigen kann.

7. Rückkopplung mit Landwirten für Plausibilitätsüberprüfungen der Analyseergebnisse und zur Bestimmung der Anpassungsreaktionen bei verschiedenen Politikoptionen. Kaum eine der Studien berichtet von einer kritischen Diskussion der Ergebnisse mit der landwirtschaftlichen Praxis.

Unter den betrachteten Analysen zum MTR Milch enthalten insbesondere die Gesamtsysteme, die auf FADN-Daten oder dem Konzept typischer Betriebe basieren, interessante Elemente im Hinblick auf die gestellten Anforderungen. Bei diesen Systemen werden Datenquellen herangezogen, die bereits EU-weit verfügbar, harmonisiert und kompatibel sind. Viele der allein auf FADN-Daten basierenden Untersuchungen weisen neben der mangelnden prozessspezifischen Differenzierung oft nur eine begrenzte (praxisferne) Abbildung der betrieblichen Anpassungsreaktionen auf. Um das Anpassungsverhalten besser berücksichtigen zu können, werden Informationen aus der Praxis erforderlich.

Im Hinblick auf die Zielsetzung dieser Arbeit wird das dynamische Modell TIPI-CAL aus dem IFCN ausgewählt, um den vorgeschlagenen Ansatz der Verknüpfung einzelbetrieblicher Datensätze zu implementieren. Folgende Gründe waren ausschlaggebend: 
- In TIPI-CAL werden die Daten EU-weit und weltweit regelmäßig erhoben.

- $\quad$ TIPI-CAL ist eine einfache, nutzerfreundliche Softwarelösung. Das Vorhaben dieser Arbeit ist anhand dieses Modells am einfachsten durchführbar.

- Der Gesamtbetrieb wird abgebildet.

- TIPI-CAL ermöglicht die Ermittlung sehr detaillierter produktionstechnischer und ökonomischer Variablen.

- Stückkostenrechnungen auf Vollkostenbasis und detaillierte Abbildungen von Produktionsverfahren und Anpassungsstrategien sind möglich: betriebliche Wachstumsprozesse können realitätsnah dargestellt werden mit Hilfe des sogenannten „Praktikeransatzes“. Der nahe Bezug zur landwirtschaftlichen Praxis ermöglicht, die häufig fehlenden Informationen über voraussichtliche Anpassungsreaktionen der Betriebe bei agrarpolitischen Änderungen zu erhalten.

- TIPI-CAL ist ein Bestandteil des Gesamtsystems bestehend aus Daten und Modell, welches im Rahmen eines internationalen Netzwerkes (aus Wissenschaftlern, Beratern und Landwirten) nachhaltig weiterentwickelt wird.

- In TIPI-CAL erfolgt die Rückkopplung mit den Praktikern mit Hilfe eines sogenannten „Panels“ (Expertenrunde, siehe Kapitel 4.3) zur praxisnahen Einschätzung der Anpassungsreaktionen.

Die standardmäßige Datengrundlage des TIPI-CAL-Modells bilden die Datensätze typischer Modellbetriebe aus dem IFCN-Netzwerk (HEMME, 2002). Diese Daten sind im Hinblick auf das Anforderungsprofil harmonisiert und ausreichend tief gegliedert, genügen jedoch nicht der Forderung nach Repräsentativität.

Ebenfalls harmonisiert sind die EU-weit verfügbaren FADN-Datensätze (VARD, 2002), die im Rahmen der hier beschriebenen Modellierungsarbeiten zur EU-Milchmarktpolitik Verwendung fanden. Diese Daten sind im Hinblick auf das Anforderungsprofil repräsentativ, genügen jedoch nicht der Forderung nach ausreichender Datentiefe.

Dies deutet bereits auf die Schwierigkeiten einer geeigneten Datenbasis für die betriebliche Modellierung hin, worauf in Kapitel 4 ausführlich Bezug genommen wird. 


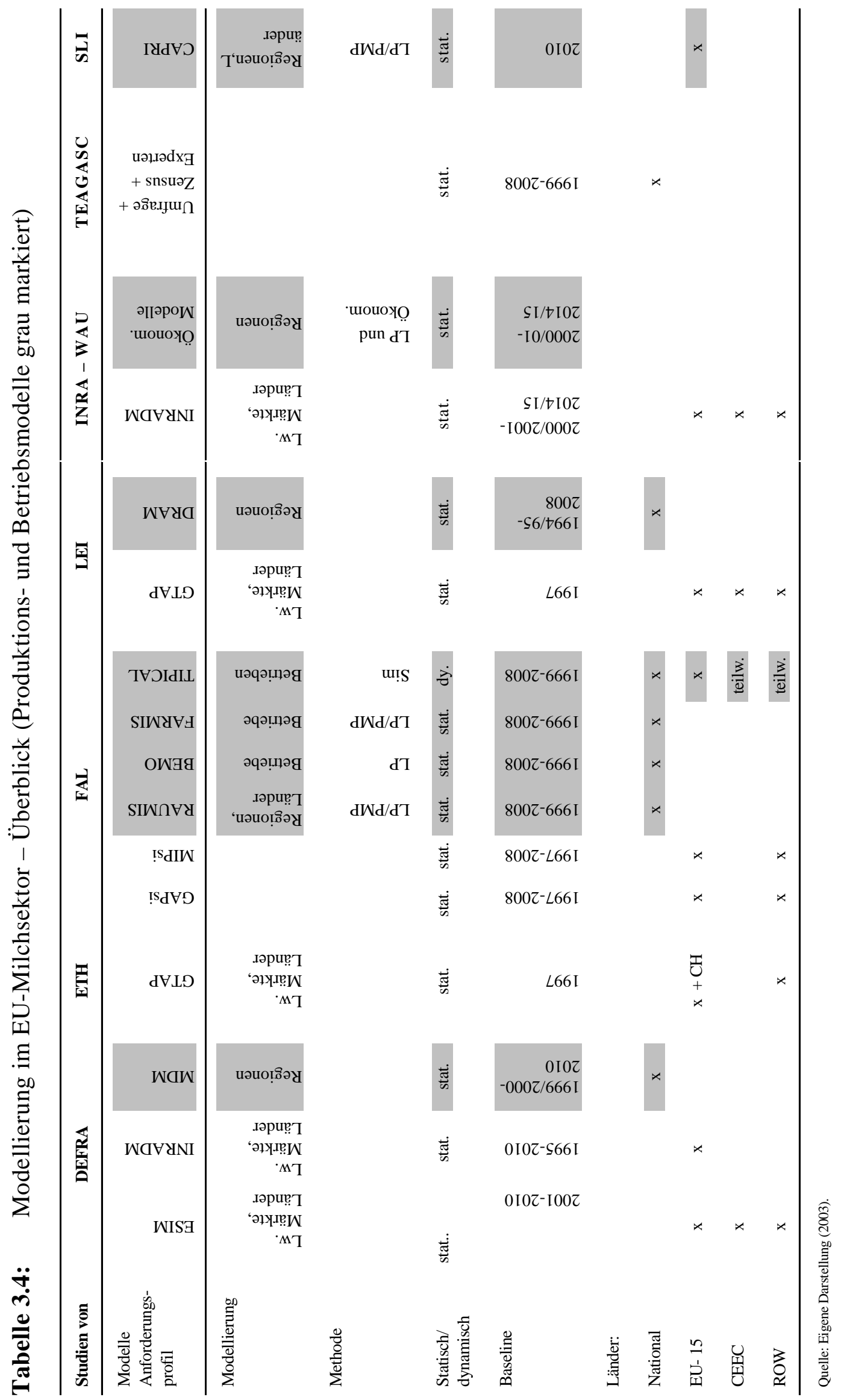




\subsection{Schlussfolgerungen}

Kapitel 3 gibt einen Überblick über den Anwendungsstand quantitativer Politikfolgenanalysen am Beispiel des EU-Milchmarktes. Die verschiedenen Modellanalysen wurden im Hinblick auf die Eignung für die Fragestellung dieser Arbeit überprüft. Aus den Schwachstellen bisheriger Analysen wurden Anforderungen an das zu entwickelnde Konzept abgeleitet.

Als besondere Schwachstelle ist herauszustellen, dass die meisten der angewendeten Modellansätze der Anforderung, betriebliches Anpassungsverhalten möglichst repräsentativ und zugleich realitätsnah darzustellen, nicht genügen. Bereits JACOBS (1998) stellte hinsichtlich zukünftigen Forschungsbedarfs die Notwendigkeit heraus, zusätzliche Kenntnisse über das landwirtschaftliche Anpassungsverhalten bereitzustellen. Die Steuerung des landwirtschaftlichen Anpassungsverhaltens ist JACOBS zufolge ein wesentlicher Modellbestandteil mit erheblichem Einfluss auf die Modellergebnisse. Auch der Modellüberblick konnte aufzeigen, dass der zukünftige Produktionsumfang bei betrieblichen und regionalen Programmierungsmodellen in der Regel nach der Verhaltenshypothese der Gewinnmaximierung optimiert wird. JACOBS konstatierte, dass die Modellergebnisse häufig nicht den beobachteten Produktionsumfängen entsprechen. Abhilfe würden u. a. systematische Befragungen von Praktikern nach ihren zukünftigen Anpassungsalternativen schaffen (JACOBS, 1998).

Im Hinblick auf die Problemstellung dieser Arbeit hat die Prüfung der für die Politikfolgenanalyse im Bereich Milch verwendeten Modelle ergeben, dass das dynamische Modell TIPI-CAL zur Durchführung des Vorhabens dieser Arbeit - zur Verknüpfung einzelbetrieblicher Datensätze - besonders geeignet ist. Dieses Modell genügt jedoch für sich alleine genommen auch nicht dem Anforderungsprofil aufgrund fehlender Repräsentativität der verwendeten Datenbasis. Das bedeutet für diese Arbeit, dass TIPI-CAL noch mit einem repräsentativen Datensatz, der möglichst EU-weit harmonisiert erhoben wird, zu erweitern ist. Daher wird in dieser Arbeit der Versuch unternommen, TIPI-CAL und einen repräsentativen und EU-weit harmonisierten Datensatz miteinander zu vernetzen, um zu weiterführenden Ergebnissen zu kommen.

Der Modellüberblick zeigt, dass eine Reihe von Verknüpfungen zwischen etablierten Betriebs- und Marktmodellen zu finden sind (z. B. im Hinblick auf Preisprojektionen), bisher aber keine Verknüpfungen zwischen Betriebsmodellen untereinander vorliegen. Deswegen beschäftigt sich diese Arbeit genau mit diesem Thema.

Im Hinblick auf das entwickelte Anforderungsprofil wird im folgenden Kapitel eine geeignete Datengrundlage für das Vorhaben dieser Arbeit bestimmt. 


\section{Einzelbetriebliche Datengrundlage in der EU}

Die kontinuierliche Anwendung von Betriebsmodellen zur quantitativen Folgenabschätzung in der Agrarpolitik erfordert eine Datengrundlage, die anhaltend und in gleichbleibender Qualität zur Verfügung steht. Die größte Kontinuität der Datenbereitstellung wird durch staatlich angeordnete Statistiken erreicht, die größtenteils auf dem Informationsbedarf basieren, der sich aus dem Landwirtschaftsgesetz und der Gründung der Europäischen Wirtschaftsgemeinschaft ergibt (vgl. BundESGESETZBLATT vom 05.09.1955, Teil 1, S. 565; BundESGESETZBLATT vom 25.03.1957, Teil 2, S. 766).

In Kapitel 4 erfolgt zunächst ein Überblick über die wichtigsten gesetzlich vorgeschriebenen Erhebungen und ergänzenden Datenquellen in Deutschland und in der EU, die für einzelbetriebliche Modellanalysen verwendet werden können. Anschließend werden in Kapitel 4.2 und 4.3 zwei Datensätze vorgestellt, die im Hinblick auf das in Kapitel 3 entwickelte Anforderungsprofil am geeignetesten für das Vorhaben dieser Arbeit erscheinen (FADN und IFCN).

\subsection{Datengrundlagen in der quantitativen Politikfolgenabschätzung - ein Überblick}

Im Folgenden wird zunächst ein kurzer Überblick über die Datengrundlagen gegeben, die im Rahmen von Analysen zur Änderung der Agrarpolitik (vgl. Kapitel 3) häufig genutzt werden. Die Datensätze FADN und IFCN werden in gesonderten Kapiteln vorgestellt, da sie spezifisch für diese Arbeit von Bedeutung sind.

Als erstes ist die amtliche Agrarstatistik zu erwähnen, die ein Bestandteil aus dem Erhebungsprogramm des Statistischen Bundesamtes ist. Die Daten werden durch die untergeordneten Landesämter erhoben. Gegenstand der Erhebung sind die jährlichen oder mit kürzerer Periodizität stattfindenden Allgemeinen Erzeugungsstatistiken (Agrarfachstatistiken) und die in jährlichen, zweijährlichen oder längerfristigen Abständen durchzuführenden Betriebsstatistiken.

Tabelle 4.1 zeigt die wichtigsten Agrarfach- und Betriebsstatistiken. Insgesamt werden folgende sechs Agrarstatistiken unterschieden, die sich jeweils aus mehreren Einzelerhebungen zusammensetzen (siehe Tabelle 4.1): Bodennutzungshaupterhebung, Viehzählung,

Die Erhebungen und deren Organisation werden in dem Gesetz über Agrarstatistiken (AgrStatG) in Verbindung mit dem Gesetz über die Statistik für Bundeszwecke (BstatG) sowie mehrerer Einzelgesetze geregelt. Mitte 1998 ist eine Neufassung des Agrarstatistikgesetzes in Kraft getreten. 
Arbeitskräfteerhebung, Agrarberichterstattung (AB), Landwirtschaftszählung (LZ) und andere Agrarfachstatistiken.

Tabelle 4.1: $\quad$ Überblick über Agrarstatistiken in Deutschland und in der EU

\begin{tabular}{|c|c|c|}
\hline & Deutschland & EU \\
\hline Struktur der Landwirtschaft & $\begin{array}{l}\text { a) LZ Vollerhebung etwa alle } 10 \text { Jahre } \\
\text { AB/LZ Agrarberichterstattung: bei } \\
\text { Bedarf zwischendurch Stichproben } \\
\text { b) Einzelaspekte } \\
\text { Bodennutzungshaupterhebung (jährlich) } \\
\text { Arbeitskräfteerhebung (jährlich) } \\
\text { Viehzählung (jährlich im Dezember) }\end{array}$ & $\begin{array}{l}\text { EU-Strukturerhebung (EUROSTAT) } \\
\text { (zweijährlich) }\end{array}$ \\
\hline $\begin{array}{l}\text { Einkommenslage } \\
\text { (Buchführung) }\end{array}$ & $\begin{array}{l}\text { a) Testbetriebsnetz (jährlich) } \\
\text { b) Deutscher Bauernverband } \\
\text { und LAND-DATA } \\
\text { DBV-Situationsberichte }\end{array}$ & FADN (jährlich) \\
\hline Markt- und Preisberichterstattung & ZMP (fortlaufend) & ZMP (fortlaufend) \\
\hline Sektorale Einkommensrechnung & $\begin{array}{l}\text { Landwirtschaftliche Gesamtrechnung } \\
\text { (LGR/jährlich) } \\
\text { SPEL-Daten (jährlich) }\end{array}$ & $\begin{array}{l}\text { Landwirtschaftliche Gesamtrechnung } \\
\text { (LGR/jährlich) } \\
\text { SPEL-/EU-Daten (jährlich) }\end{array}$ \\
\hline $\begin{array}{l}\text { Produktion, Handel, Nahrungsmittel- } \\
\text { bilanzen, Landnutzung, Düngung, Forst, } \\
\text { Fischerei, Bevölkerung, etc. }\end{array}$ & FAOSTAT & FAOSTAT (international harmonisiert) \\
\hline
\end{tabular}

Quelle: Eigene Zusammenstellung.

Eine Sonderstellung nimmt die Agrarberichterstattung und die Landwirtschaftszählung ein. Beide Agrarfachstatistiken zählen zu den Betriebsstatistiken. Ihre Hauptaufgabe besteht in der Zusammenführung betrieblicher Informationen aus anderen bereits vorliegenden Erhebungen. Durch die Gruppierung von Betrieben sollen strukturelle Veränderungen wie Art und Umfang der Bodennutzung, wirtschaftliche Ausrichtung, Betriebsgröße oder sozioökonomische Kennzeichnung der Betriebe analysiert werden. ${ }^{2}$

2 Grundlagen der Betriebsstatistiken sind die etwa alle zehn Jahre - häufig im Rahmen weltweiter Agrarzensen - stattfindenden Landwirtschaftszählungen (LZ) einschließlich ihrer Einzelerhebungen über Weinbau, Gartenbau und Binnenfischerei sowie die Agrarberichterstattung (AB), die im zweijährigen Turnus angesetzt und seither regelmäßig (außer 1981) mit EU-Strukturerhebungen verknüpft sind. In die AB bzw. LZ werden betriebsbezogene Ergebnisse aus der Arbeitskräfteerhebung in der Landwirtschaft, aus der Dezember-Viehzählung und der Bodennutzungshaupterhebung übernommen (Grundprogramm). Zusätzlich enthält die AB/LZ auch Merkmale, die im Rahmen eines Ergänzungsprogramms und bei Bedarf eines Zusatzprogramms erhoben werden (vgl. HASSKAMP, 1976). 
Im Folgenden werden die der Betriebsstatistik zuzuordnenden Datenquellen näher vorgestellt und hinsichtlich ihrer Verwendung eingeordnet:

Die Landwirtschaftszählung wird in ca. 10-jährigen Abständen auf Empfehlung der FAO zur Weltlandwirtschaftszählung und zur Unterstützung der EU-Strukturerhebung durchgeführt (vgl. HASSKAMP, 1979, S. 245-253). Sie besteht im Kern aus einer Haupterhebung, die sich aus den Merkmalen der Agrarberichterstattung und aus sonstigen Merkmalen zusammensetzt. Die letzte Landwirtschaftszählung erfolgte im Frühjahr 1999. Sie stellt die umfangsreichste und wichtigste Informationsquelle über die Produktionsgrundlagen sowie die strukturelle, wirtschaftliche und soziale Situation der land- und forstwirtschaftlichen Betriebe dar.

Die ab 1975 zweijährlich aufgrund eigener Rechtsgrundlagen durchzuführenden EUStrukturerhebungen (EUROSTAT) sollen über Art und Umfang der vielfältigen Erscheinungsformen des Strukturwandels Aufschluss geben. Damit sollen sie den wachsenden Bedarf der zuständigen Ressorts des Bundes und der Länder, der EU-Gremien, der Wissenschaft, der Verbände sowie der Wirtschaft und der Verwaltung an zeitnahen, sachlich und regional tiefgegliederten Strukturdaten abdecken (vgl. STATISTISCHES BundESAMT WIESBADEN, 1979, S. 1).

Die Strukturerhebungen in land- und forstwirtschaftlichen Betrieben (Agrarstrukturerhebung, Landwirtschaftszählung) wurden mit der Novellierung des Agrarstatistikgesetzes ${ }^{3}$ im Jahr 1998 erstmals in einem gemeinsamen Abschnitt geregelt und sie umfassen nun folgende Einzelerhebungen (STAtistisches Bundesamt WiesBaden, 1999, S. 2).

- EU-Agrarstrukturerhebung (ASE) bestehend aus dem Grundprogramm und Ergänzungsprogramm. Sie werden gemeinsam durchgeführt. Diese neue Organisationsform der „integrierten Erhebung“ ist im Grundsatz die enge Verzahnung der Produktionsund Strukturstatistiken bei einem einheitlichen Betriebskreis. Die Agrarstrukturerhebung wird alle zwei Jahre im Wechsel allgemein und repräsentativ durchgeführt.

- EU-Landwirtschaftszählung bestehend aus der Haupterhebung, Weinbau-, Gartenbauund Binnenfischereierhebung. Die Haupterhebung der Landwirtschaftszählung wurde gemeinsam mit der Agrarstrukturerhebung im ersten Halbjahr 1999 durchgeführt.

Die Bundesrepublik Deutschland liefert an das Statistische Amt der Europäischen Gemeinschaften (EUROSTAT) im Rahmen der EU-Agrarstrukturerhebung Ergebnisse über die Besitz- und Eigentumsverhältnisse der landwirtschaftlichen Betriebe. Die EUAgrarstrukturerhebung ist in die Agrarberichterstattung integriert. Das Erhebungspro-

Gesetz über Agrarstatistiken (Agrarstatistikgesetz, AgrStatG) in der Fassung der Bekanntmachung vom 25. Juni 1998, BGBI. I, S. 1635. 
gramm von EUROSTAT stimmt im Wesentlichen mit dem der Agrarberichterstattung überein. Die Definitionen und Erhebungsbereiche sind aufeinander abgestimmt.

EUROSTAT hat die Aufgabe, die Europäische Union mit qualitativ hochwertigen statistischen Informationen zu versorgen. Es ist damit beauftragt, die harmonisierten Statistiken der Europäischen Union zu veröffentlichen.

Die Agrarberichterstattung wurde ab 1975 eingeführt und mit einer Periodizität von zwei Jahren durchgeführt. Die Bundesregierung hat gemäß § 4 LwG. jährlich dem Deutschen Bundestag und dem Bundesrat einen „Bericht über die Lage der Landwirtschaft“ einschließlich einer darin enthaltenen „Stellungnahme“ zum erzielten Vergleichseinkommen vorzulegen. ${ }^{4}$

Neben den umfangreichen Einkommensanalysen auf Basis des Testbetriebsnetzes enthält die Agrarberichterstattung detaillierte statistische Daten volkswirtschaftlicher und agrarsektoraler Art. Sie alle dienen zur umfassenden Information über die Lage der Landwirtschaft und ihre Ursachen, über Ernten und Preise, strukturelle Veränderungen, ihre Investitionen und die Vermögensentwicklung.

Repräsentativ werden im zweijährigen Abstand ausgewählte landwirtschaftliche Betriebe („Stichprobenbetriebe“) befragt. Die Erhebung der Auswahlbetriebe erfolgt in Form einer Stichprobe entsprechend den Richtlinien des Bundesministeriums zur Testbuchführung, dem Landwirtschaftsgesetz von 1955 sowie im Einvernehmen mit dem Beirat zur Feststellung der Ertragslage gemäß § 3 LwG (1982). Erhoben werden sämtliche Merkmale des Grundprogramms (Bodennutzung, Viehbestände, Arbeitskräfte) sowie des Ergänzungsprogramms (Eigentums- und Pachtverhältnisse, Erwerbs- und Unterhaltsquellen, Buchführung, sozialökonomische Verhältnisse, Anfall tierischer Exkremente). Zusätzlich werden in jedem vierten Jahr außer den Stichprobenbetrieben auch alle übrigen landwirtschaftlichen Betriebe und alle Forstbetriebe in die Erhebung einbezogen (Totalerhebung).

Das sogenannte nationale Testbetriebsnetz („Netz der Stichprobenbetriebe“) soll die Einkommenslage der Landwirtschaft repräsentativ abbilden und die ganze Vielfalt der heutigen Betriebsformen und Bewirtschaftungsverhältnisse darstellen. Die repräsentative Aus-

In der Stellungnahme ist darzulegen, inwieweit ein den Löhnen vergleichbarer Berufs- und Tarifgruppen entsprechender Lohn für die fremden und familieneigenen Arbeitskräfte (umgerechnet auf notwendige Vollarbeitskräfte), ein angemessenes Entgeld für die Tätigkeit des Betriebsleiters (Betriebsleiterzuschlag) und eine angemessene Verzinsung des betriebsnotwendigen Kapitals erzielt worden sind. 
wahl wird gemäß den BMELF-Richtlinien von speziellen Landesausschüssen nach einheitlichen Kriterien vorgenommen. ${ }^{5}$

Erhebungsgrundlage für die Buchführungsergebnisse der Testbetriebe ist der BMELFJahresabschluss (BMELF, 1993, S. 110 ff.). Die Vermögensbewertung folgt den steuerlichen Ansätzen. Im Mittelpunkt der Buchführungsauswertungen steht die in Staffelform angelegte Gewinn-und-Verlust-Rechnung für das landwirtschaftliche Unternehmen nach dem Gesamtkostenverfahren. Zur Beurteilung der sozialen Lage der landwirtschaftlichen Familien werden darüber hinaus auch die Gesamteinkommen und die verfügbaren Einkommen des Betriebsinhabers und seines Ehegatten errechnet.

Die festgelegte Abgrenzung der Positionen im Abschluss führt zu einer Vereinheitlichung der Begriffe und ermöglicht Betriebsvergleiche innerhalb der in der Landwirtschaft anzutreffenden Rechtsformen sowie mit anderen Betrieben der gewerblichen Wirtschaft. Zudem haben Bund und Länder die Definitionen und die Abgrenzungen der Haupterwerbsbetriebe, der Betriebsformen und der Größenklassen an die EU-Typologie angepasst. Damit wird insbesondere die Vergleichbarkeit mit ähnlichen Statistiken der EU und mit den Buchführungsstatistiken anderer Mitgliedstaaten verbessert.

Das deutsche Testbetriebsnetz weist mit 9.000 Variablen je Betrieb (physische/monetäre Größen inkl. Informationen über Standort- und Strukturmerkmale, Betriebsleiterfähigkeiten, etc.) eine bemerkenswerte Datenbreite und -tiefe auf. Insgesamt beinhaltet das deutsche Testbetriebsnetz ca. 10.000 Betriebe. Die Daten liegen auf gesamtbetrieblicher Ebene vor.

Im Vergleich zu den nationalen Testbetriebsnetzen ist das Ziel des europäischen Testbetriebsnetzes (FADN), die Einkommenslage der Landwirtschaft europaweit abzubilden. Um den unterschiedlichen Strukturen des Agrarsektors in der Europäischen Union gerecht zu werden, weist das FADN im Vergleich zum nationalen Testbetriebsnetz einige Besonderheiten auf, die in Kapitel 4.2.3 ausführlich beschrieben werden.

Neben den Grunddaten der allgemeinen Statistik werden vom Deutschen Bauernverband (DBV) in sogenannten „Situationsberichten“ insbesondere ausgewählte Buchführungsergebnisse dargestellt. Die Unterlagen werden von der LAND-DATA bereitgestellt und ausgewertet und für spezielle Analysen (u. a. auch bei der Bundesforschungsanstalt für Landwirtschaft) genutzt. Darin enthalten sind Buchführungsabschlüsse vom landwirtschaftlichen Buchführungsverband für Schleswig-Holstein und Hamburg in Kiel sowie

5 Unter Vorsitz eines Vertreters der obersten Landesbehörde für Landwirtschaft gehören dem Ausschuss an: je ein Vertreter der Landwirtschaftskammern, in Ländern ohne Landwirtschaftskammern ein Vertreter der Mittelbehörde, ein Vertreter der Finanzverwaltung, ein Vertreter des Berufsstandes und ein Vertreter der landwirtschaftlichen Buchstellen. Zusätzlich können Sachverständige gehört werden. 
vom Rheinischen Landwirtschaftsverband gelieferte Buchführungsabschlüsse. Bei vergleichsweise hoher Betriebszahl werden keine vollständigen Stichproben abgebildet und keine Zufallsauswahl vorgenommen, so dass die Ergebnisse trotz Hochrechnung für die Haupterwerbsbetriebe insgesamt nicht wirklich repräsentativ sind (BRANDKAMP, 1984, S. 144-145).

Erklärtes Ziel verschiedener regionaler Einkommensanalysen ${ }^{6}$ ist es, vorzugsweise für Landwirte und Berater sowie für regionale Institutionen brauchbare Ergebnisse zu liefern, auch wenn die Kammer- und Ländergrenzen überschreitende Vergleichbarkeit durchaus angestrebt wird (vgl. Dissertation REIL, 2004).

Obwohl die Betriebe bei keiner dieser Regionalanalysen nach statistischen Zufallskriterien ausgewählt werden, ermöglicht die relativ große Zahl der dargestellten Betriebsergebnisse stärkere Regionalisierungen des Materials und der Aussagen, als sie im Agrarbericht der Bundesregierung erforderlich und möglich sind. Länderministerien und Landwirtschaftskammern haben spezifische regionale Aufgabenstellungen und benötigen deshalb eine entsprechend umfangreiche Materialgrundlage, wie sie nach dem Landwirtschaftsgesetz nicht vorgesehen und zur Erfüllung des gesetzlichen Auftrages auch nach stichprobentheoretischen Grundsätzen nicht erforderlich ist (BRANDKAMP, 1984, S. 129-152).

Die Vielzahl regional geführter Datenbestände ${ }^{7}$ in den einzelnen EU-Ländern, teils mit Betriebszweigabrechung, hat jedoch den Nachteil, dass sie unterschiedlich tief gegliedert sind und häufig keine Angaben zu unternehmenseigenen Faktoren bieten. Die verschiedenen Datensätze sind vielfach nur unzureichend harmonisiert. Sie unterscheiden sich in der Datentiefe, im Bezugszeitraum und in der Erhebungsmethodik (z. B. Abschreibungsmethoden, die Erfassung und Bewertung der Arbeitszeit und die gesonderte Erfassung von Vorleistungen und -preisen).

Neben den Daten aus den Betriebsstatistiken werden insbesondere für Projektionsrechnungen auch die Marktdaten der ZMP bei Politikfolgenabschätzungen genutzt. Aufgabe der ZMP (Zentrale Markt- und Preisberichtstelle der Land-, Forst- und Ernährungswirtschaft $\mathrm{GmbH}$ ) ist es, über die Lage an den nationalen und internationalen Märkten für Agrar- und Ernährungsgüter zu berichten, um die Markttransparenz zu verbessern und somit die Wettbewerbsfähigkeit der deutschen Agrarwirtschaft im internationalen Warenhandel zu stärken.

6 Deutschland: z. B. Beratungsringdaten, BZA-Rind/Schleswig-Holstein, BZA der Landwirtschaftskammer Rheinland, Rinderreport Baden Württemberg, Rinderreport Bayern etc. 
Das Augenmerk der ZMP gilt insofern zu einem großen Teil den landwirtschaftlichen Erzeugerpreisen. Die Entwicklungen auf den nachgelagerten Stufen bis hin zum Lebensmitteleinzelhandel werden ebenfalls von der ZMP beobachtet, ausgewertet und neben den Marktteilnehmern und Verbrauchern auch der Wissenschaft zur Verfügung gestellt. Die ein- bis mehrmals wöchentlich erscheinenden Marktberichte liefern Informationen aus allen Bereichen der Agrarwirtschaft. Hintergrundberichte, Analysen und Prognosen geben ein Feedback zur Beurteilung des kurz- bis langfristigen Marktgeschehens in Deutschland, der Europäischen Union und der Welt. ${ }^{8}$

Die landwirtschaftliche Gesamtrechnung (LGR) dient als Grundlage für Analysen, Vorausschätzungen und politische Maßnahmen. Sie soll als nationale Gesamtrechnung einen systematischen, vergleichbaren und möglichst vollständigen Überblick über die Tätigkeiten in der Landwirtschaft geben. Die Vielzahl der wirtschaftlich relevanten Transaktionen und der an ihnen beteiligten Einheiten wird nach einheitlichen Gesichtspunkten geordnet und in einem konsistenten System von Tabellen dargestellt (vgl. EUROSTAT, 1989).

Abschließend sind die SPEL- und FAO-Daten zu nennen. SPEL ${ }^{9}$ ist ein Gemeinschaftsprojekt des Lehrstuhls für Volkswirtschaftslehre, Agrarpolitik und Landwirtschaftliches Informationswesen der Universität Bonn, des „European Center for Agricultural, Regional and Environmental Policy Research, EuroCARE, Luxembourg/Bonn und der Europäischen Kommission, GD 34, (SPEL-Gruppe in EUROSTAT), Luxembourg. Die Arbeiten an der Datenbasis mündeten im Basissystem „SPEL/EU-BS“ (vgl. WOLF, 1995). Dieses kombiniert Daten aus verschiedenen Quellen, erstellt eine differenzierte und konsistente Expost-Abbildung der Struktur, Intensität und Verwendung der landwirtschaftlichen Produktion sowie der Einkommensentstehung in den landwirtschaftlichen Sektoren der EUMitgliedstaaten und stimmt in Gesamtgrößen mit der Landwirtschaftlichen Gesamtrechnung (LGR) überein. Das SPEL-/EU-System umfasst die landwirtschaftlichen Sektoren von fünfzehn Mitgliedstaaten und der EU als Ganzes und dient als Grundlage für das Kurzfristprognose- und Simulationssystem (SPEL/EU-SFSS).

Die FAO (Food and Agriculture Organization of the United Nations) wurde 1945 gegründet und ist eine der größten spezialisierten Vertretungen im System der Vereinten Nationen und die führende Vertretung für Land- und Forstwirtschaft, Fischerei und ländliche Entwicklung. FAOSTAT ist eine „online“ zugängliche mehrsprachige Datenbank, die über 1 Million Zeitreihenaufzeichnungen enthält und international harmonisierte Statistiken in den folgenden Bereichen abdeckt und u. a. der Wissenschaft zur Verfügung stellt: Produktion,

${ }^{8} \quad$ Detailliertere Informationen zu den wöchentlichen Marktberichten und Jahresberichten enthält folgende Homepage: www.zmp.de.

9

SPEL= Sektorales Produktions- und Einkommensmodell für die Landwirtschaft. 
Handel, Nahrungsmittelbilanzen, Dünger und Schädlingsbekämpfungsmittel, Landnutzung und Bewässerung, Forstprodukte, Fischereiprodukte, Bevölkerung, Landwirtschaftliche Maschinen, Lebensmittelhilfstransporte.

In den folgenden Kapiteln 4.2 und 4.3 werden die Datensätze FADN und IFCN, die für diese Arbeit von besonderem Interesse sind, ausführlich vorgestellt.

\subsection{Konzept der Europäischen Testbetriebsstatistik (FADN)}

Da die FADN-Datenbasis eine einzelbetriebliche Datengrundlage darstellt, die besonders wichtige Merkmale wie die Kontinuität und anhaltende Qualität der Erhebung, aber auch die EU-weite Harmonisierung aufweist, wurde diese Datengrundlage für das Vorhaben dieser Arbeit neben der IFCN-Datenbasis ausgewählt. Im Folgenden wird das Erhebungsprogramm dieser Datenbasis ausführlich vorgestellt. Gegenstand der Darstellung sind zunächst die Zielsetzung und die Entwicklung des europäischen Testbetriebsnetzes. Anschließend werden das Erhebungsprogramm und die Unterschiede zum nationalen Erhebungsprogramm der Testbetriebsstatistik erläutert.

\subsubsection{Zielsetzung}

Die Gründung des Informationsnetzes landwirtschaftlicher Buchführungen (INLB, englische Bezeichnung: FADN) für die EWG wurde im Jahre 1965 vom Rat der europäischen Wirtschaftsgemeinschaft beschlossen (Verordnung Nr. 79/65/EWG des Rates). Schon damals war es das Ziel, mit Hilfe des FADN für die Gestaltung der gemeinsamen Agrarpolitik objektive und zweckdienliche Informationen über die Lage der Landwirtschaft, insbesondere über die Einkommen in den verschiedenen landwirtschaftlichen Betriebsgruppen, zu erhalten.

Das Informationsnetz enthält jährlich eine ausgewählte Anzahl von landwirtschaftlichen Buchführungsbetrieben der Europäischen Union, und es liefert die Angaben, die als Grundlage für die Berichte der Kommission über die Lage der Landwirtschaft, insbesondere der Einkommen, herangezogen werden. Viele Jahre lang dienten die Auswertungen des Informationsnetzes der Kommission, dem Rat und dem europäischen Parlament als Entscheidungshilfe und außerdem für die jährlichen Vorschläge zur Festsetzung der Preise verschiedener Marktordnungsprodukte. 


\subsubsection{Entwicklung}

Zur Durchführung der Tätigkeit des Informationsnetzes wurde eine Reihe von Regelungen getroffen, die eine Erhebung und Klassifizierung der Betriebe der Gemeinschaft nach einheitlichen Grundsätzen gewährleisten. Besonders die Einführung des gemeinschaftlichen Betriebsbogens (Verordnung (EWG) Nr. 2237/77 der Kommission) und die Schaffung eines standardisierten Klassifizierungssystems (Entscheidung Nr. 78/463/EWG der Kommission) stellen die Grundlagen für eine einheitliche Behandlung aller Betriebe der Gemeinschaft dar. Diese Grundsätze sind von besonderer Bedeutung, weil die Kommission selber keine eigenen Datenerhebungen durchführt. Diese Aufgabe nehmen Behörden und Institute in den Mitgliedstaaten wahr. Die Kommission erhält für mehrere Mitgliedstaaten nur eine Auswahl von Buchführungsbetrieben, die auf nationaler Ebene teilweise detailliertere Informationen enthalten oder nach anderen Zielsetzungen erhoben werden. So enthält beispielsweise das Deutsche Testbetriebsnetz ca. 9.000 Variablen je Betrieb, die EUFADN-Daten maximal 1.866 Variablen je Betrieb.

Zur Einhaltung der gemeinsamen Richtlinien wurde ein weitverzweigtes Konsultationsund Beratungssystem aufgebaut (siehe Abbildung 4.1). Die wichtigsten Harmonisierungen und Absprachen zwischen den Erfordernissen des FADN und den nationalen Erhebungen werden mehrmals jährlich auf den Sitzungen des Gemeinschaftsausschusses des Informationsnetzes vorgenommen, der sich aus Vertretern der Mitgliedstaaten und der Kommission zusammensetzt. Hier erfolgen nicht nur die notwendigen Koordinierungen und Diskussionen der laufenden Auswertungen, sondern auch die Aussprachen über wünschenswerte Veränderungen des Informationsnetzes.

1995 wurde das Projekt „PACIOLI“ ins Leben gerufen. ${ }^{10}$ Die „PACIOLI“-Projektgruppe besteht aus Experten aus den 15 EU-Mitgliedstaaten als auch aus den Beitrittsländern. Sie trägt zur Entwicklung von Informationssystemen bei, die das FADN-Netzwerk mit Daten verschiedenster Art verbessern und erweitern sollen. Damit soll der politische Entscheidungsprozess und die Bewertung auf EU- und nationaler Ebene unterstützt werden.

Seit einigen Jahren wird das FADN auch für Politikfolgenanalysen verwendet. Die für Prozessanalysemodelle erforderlichen prozessspezifischen Daten liegen jedoch zum überwiegenden Teil nicht vor, so dass diese mittels geeigneter Verfahren generiert werden müssen. Theologie und Mathematik. PACIOLI schrieb das bedeutende mathematische Werk „Summa de Arithmetica, Geometria, Propotioni et Propotionalita“ (1494). Dessen 11. Traktat enthält die systematische Darstellung der doppelten Buchhaltung, die die gesamte nachfolgende Buchhaltungsliteratur Italiens und Mitteleuropas bedeutend beeinflusst hat. Eine deutsche Übersetzung des 11. Traktats erschien 1933 mit umfassender historischer Einleitung. Der 4. Traktat behandelt den Wechsel und den Zahlungsverkehr. 
Abbildung 4.1: Organisation des Informationsnetzes landwirtschaftlicher Buchführungen

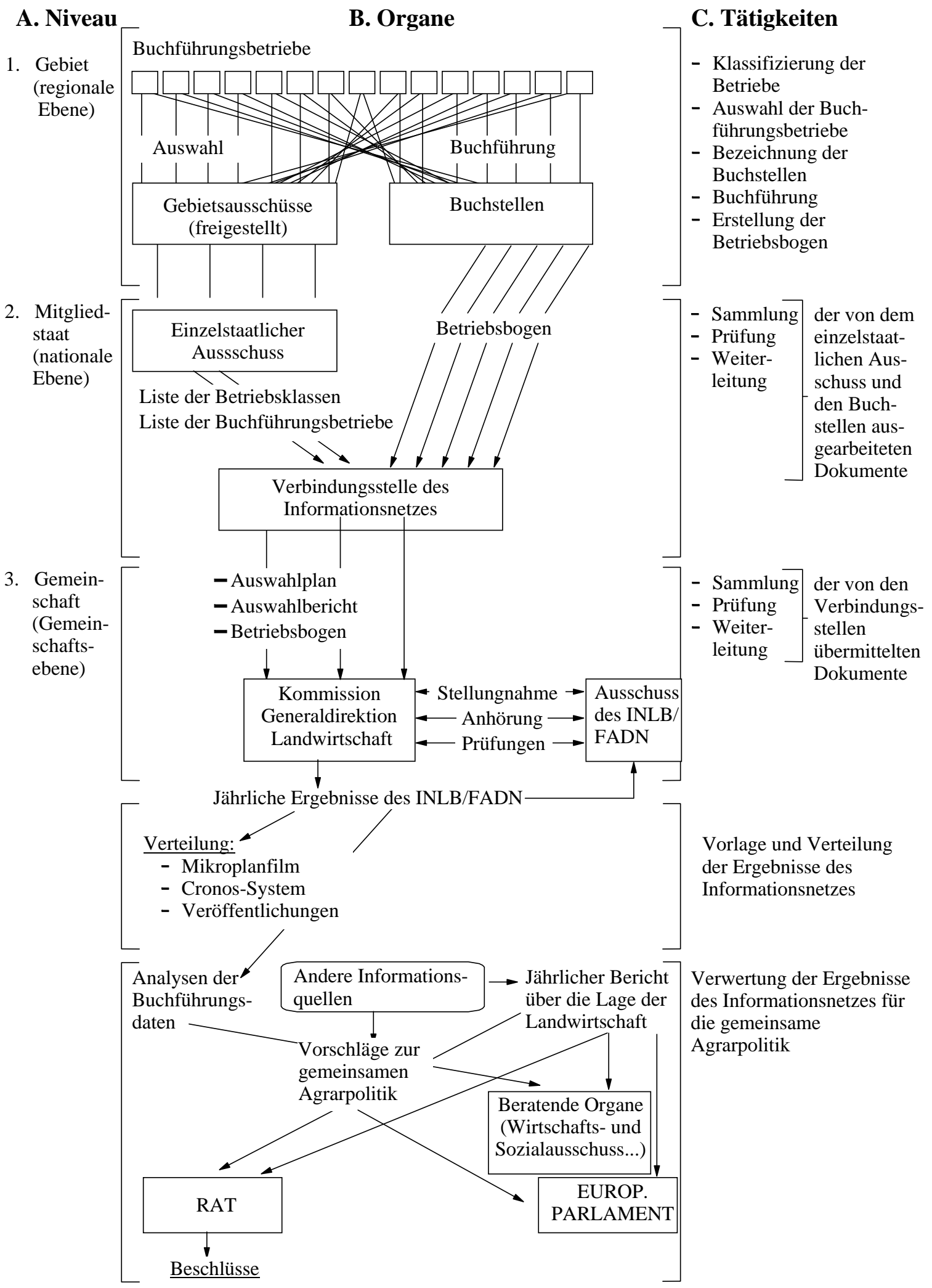

Quelle: Lommez, Jan M.J. (1984). 
Erschwerend für die Arbeit des FADN war und ist die unterschiedliche Ausgangslage für die Erhebung von Buchführungsdaten in den Mitgliedsstaaten. Während einige Länder wie das Vereinigte Königreich, die Niederlande oder Deutschland über eine vergleichsweise lange Erfahrung und gute Organisation in der landwirtschaftlichen Buchführung verfügen, musste in anderen Mitgliedstaaten eine völlig neue Organisation in einer nicht immer buchführungsfreundlichen Landwirtschaft aufgebaut werden. Während in Deutschland ein föderales System vorliegt, findet beispielsweise in Frankreich eine zentrale Verwaltung statt. Außerdem stellte die EU-Osterweiterung weitere Anforderungen. Die gemeinsamen Entscheidungen zum reibungslosen Funktionieren des Informationsnetzes stellen deshalb nicht immer das Optimum eines denkbaren theoretischen Ansatzes dar, sondern sie stützen sich vielmehr auf die Möglichkeiten aller Mitgliedstaaten, gleichermaßen die benötigten Informationen zusammenzustellen.

\subsubsection{Stichprobe landwirtschaftlicher Betriebe im EU-Testbetriebsnetz}

Das europäische Testbetriebsnetz weist im Vergleich zu den nationalen Testbetriebsnetzen einige Besonderheiten auf, die sich vor allem aus den unterschiedlichen Strukturen des Agrarsektors in der Gemeinschaft ergeben. Im Folgenden wird kurz auf diese Merkmale und auf die Auswahl der Betriebe eingegangen.

\section{Schichtung der Grundgesamtheit}

Um den unterschiedlichen Strukturen des Agrarsektors in der Europäischen Union gerecht zu werden, die eine weitaus größere Diversität aufweist als die der einzelnen Mitgliedstaaten, wurde insbesondere für die Bedürfnisse des FADN und die gemeinschaftlichen Strukturerhebungen ein spezielles Klassifizierungssystem geschaffen, das sich auf die betriebswirtschaftliche Ausrichtung und Betriebsgröße stützt (Entscheidung Nr. 78/463/EWG). Mit Hilfe des gemeinschaftlichen Klassifizierungssystems wird zunächst die (wirtschaftliche) Größe eines Agrarbetriebs aufgrund seiner potentiellen Bruttowertschöpfung (gesamtbetrieblicher Standarddeckungsbeitrag) ermittelt. Dann wird anhand des Anteils der einzelnen Erzeugnisse an diesem gesamtbetrieblichen Standarddeckungsbeitrag die betriebswirtschaftliche Ausrichtung (bzw. der Betriebstyp) bestimmt.

Der Standarddeckungsbeitrag (StDB) je Flächen- oder Tiereinheit ist definiert als der Wert des Ertrags je Hektar oder Nutztier abzüglich der entsprechenden variablen Spezialkosten. Für jedes Gebiet wird allen pflanzlichen und tierischen Erzeugungen ein StDB zugewiesen. Die StDB werden von den Verbindungsstellen selbst auf der Grundlage der in den landwirtschaftlichen Betrieben gesammelten Daten berechnet. Sie werden alle zwei Jahre aktualisiert und auf Gebietsebene für über 90 verschiedene Einzelerzeugungen berechnet. 
Die betriebswirtschaftliche Ausrichtung wird durch den relativen Beitrag der verschiedenen Betriebszweige zum gesamten Standarddeckungsbeitrag des Betriebes festgelegt. Ein Betrieb ist beispielsweise der betriebswirtschaftlichen Hauptausrichtung „spezialisierter Milchviehbetrieb“ zuzuordnen, wenn auf die Milchwirtschaft mehr als zwei Drittel des gesamten StDB des Betriebes entfällt.

Die regionale Gliederung des FADN orientiert sich an den üblichen Verwaltungseinheiten auf nationaler Ebene. Für die Zwecke des FADN wird zwischen 103 Regionen in der Europäischen Union unterschieden, wobei Irland, Dänemark, Belgien, Luxemburg, Österreich und die Niederlande jeweils eine Gebietseinheit repräsentieren.

Durch das spezielle Klassifizierungssystem ist es demnach möglich, zwischen maximal 72 betriebswirtschaftlichen Ausrichtungen, 9 wirtschaftlichen Betriebsgrößen und 103 Regionen zu unterscheiden. Gleichzeitig ist die Typologie jedoch so angelegt, dass neu zusammengefasste Betriebsgruppen gebildet werden können, ohne dass die Kohärenz des Klassifizierungssystems verloren geht. Das gemeinschaftliche Klassifizierungssystem wird sowohl auf die gemeinschaftlichen Strukturerhebungen, die das Statistische Amt der Europäischen Gemeinschaften in Luxemburg durchführt, als auch auf die Auswertungen des FADN angewandt. Es stellt somit das Bindeglied zwischen beiden Informationsquellen dar.

Die Auswahl der FADN-Stichprobe weist alle Merkmale eines geschichteten Auswahlverfahrens mit unterschiedlichen Auswahlsätzen auf (siehe Tabelle 4.2). Die Schichtenbildung orientiert sich an den regionalen Abgrenzungskriterien und an den durch das gemeinsame Klassifizierungssystem vorgegebenen betriebswirtschaftlichen Ausrichtungen und Größenklassen.

Tabelle 4.2: $\quad$ Schichtung der INLB-/FADN-Stichprobe

\begin{tabular}{|c|c|c|c|}
\hline $\mathbf{E U}$ & REGION & Haupt- / Einzelausrichtung & Dimension \\
\hline & 15 EU- Mitgliedstaaten ${ }^{1)} 103$ Regionen & 172 betriebswirtschaftliche Ausrichtungen & $\begin{array}{l}9 \text { wirtschaftliche } \\
\text { Betriebsgrößen }\end{array}$ \\
\hline
\end{tabular}

1) Zum Zeitpunkt der Berechnungen dieser Arbeit bestand die EU aus 15 Mitgliedsländern. Quelle: Lommez, Jan M.J. (1984).

\section{Durchführung der Auswahl}

Für jeden Mitgliedstaat ist grundsätzlich die Gesamtzahl der Auswahlbetriebe nach Maßgabe der rechtlichen, organisatorischen und zeitlichen Möglichkeiten der nationalen Verwaltungen festgelegt. Zur optimalen Verteilung der Stichprobe auf die einzelnen Regionen der EU wurden unter Beibehaltung der Gesamtzahl der Buchführungsbetriebe für jedes 
Mitgliedsland optimierte Auswahlsätze in Bezug auf die Erfassung der Streuung des Nettobetriebseinkommens berechnet. Diese Auswahlsätze fanden bei der Festlegung des Umfangs der Stichprobe teilweise Berücksichtigung (Verordnung (EWG) Nr. 1859/82 der Kommission). Als Auswahlgrundlage dienen die gemeinschaftlichen Strukturerhebungen des Statistischen Amtes der Europäischen Gemeinschaften, bei denen sichergestellt ist, dass die landwirtschaftlichen Betriebe der EU nach gemeinschaftlichen Kriterien erfasst und klassifiziert worden sind. Die eigentliche Auswahl wird auf der Grundlage des Organisationsplanes (Abbildung 4.1, siehe oben) von den Mitgliedstaaten vorgenommen. Die landwirtschaftlichen Betriebe werden in der Stichprobe nach einem Auswahlplan ausgewählt, der eine repräsentative Auswahl gewährleistet.

Die Kommission erhält vor Beginn des Rechnungsjahres einen Auswahlplan und auf diese Weise die Möglichkeit zur frühzeitigen Stellungnahme, um in Absprache mit den zuständigen Verbindungsstellen eine möglichst nach einheitlichen Kriterien durchgeführte Auswahl für alle $15^{11}$ EU-Mitgliedstaaten zu erreichen.

\section{Stichprobenumfang}

Über das FADN wird nicht die Gesamtzahl aller landwirtschaftlichen Betriebe in der Gemeinschaft, sondern nur eine Teilgesamtheit erfasst. Die Grundgesamtheit umfasst die Betriebe in der Gemeinschaft mit mindestens 1 ha landwirtschaftlicher Nutzfläche sowie diejenigen mit weniger als 1 ha, sofern sie einen bestimmten Anteil ihrer Erzeugung vermarkten bzw. einen festgelegten Erzeugungswert überschreiten.

Bei der Definition des FADN-Beobachtungsfeldes richtet sich die Kommission nach den Leitlinien der Verordnung 79/65/EWG vom 15.06.1965 und nachfolgenden Änderungen und verfolgt damit einen pragmatischen Ansatz, indem sie nur landwirtschaftliche Betriebe einschließt, die als Haupterwerbsbetriebe gelten.

Als FADN-Haupterwerbsbetriebe gelten landwirtschaftliche Betriebe, die eine bestimmte wirtschaftliche Betriebsgröße überschreiten. Die wirtschaftliche Betriebsgröße wird in Europäischen Größeneinheiten (ESU) gemessen. Zu diesem Zweck wird der in Euro ausgedrückte gesamtbetriebliche Standarddeckungsbetrag durch den Wert einer ESU geteilt. Ursprünglich entsprach eine ESU genau 1.200 €. Im Laufe der Jahre wurde der Wert einer ESU allerdings mehrfach angepasst, damit die landwirtschaftlichen Betriebe nicht infolge der Inflation künstlich „größer“ werden.

11

Zum Zeitpunkt der in dieser Arbeit durchgeführten Berechnungen bestand die EU aus 15 Mitgliedstaaten. Die FADN-Daten lagen mit einer Verzögerung von zwei Jahren nach dem EU-Beitritt vor. 
Landwirtschaftliche Betriebe, die unabhängig von etwaigen außerlandwirtschaftlichen Erwerbstätigkeiten des Betriebsinhabers eine bestimmte Betriebsgröße in ESU überschreiten, fallen also in das Beobachtungsfeld. Um den unterschiedlichen Strukturbedingungen in der Europäischen Union Rechnung zu tragen, werden jedoch für jeden Mitgliedstaat gesonderte Schwellenwerte festgelegt. Eine Auflistung dieser Schwellenwerte befindet sich in Anhangstabelle A4.1. Damit können z. B. im Falle Deutschlands neben den Haupterwerbsbetrieben auch vermehrt Nebenerwerbsbetriebe erhoben werden. Mit der Festlegung unterschiedlicher Auswahlschwellen, die den Strukturen der Agrarsektoren angepasst sind, wird ein sinnvoller Vergleich zwischen den Mitgliedstaaten ermöglicht.

Der Umfang der FADN-Stichprobe und das Durchschnittsgewicht eines Stichprobenbetriebs sind, wie nachstehend in Tabelle 4.3 ersichtlich, in den Mitgliedstaaten (EU-15) verschieden.

Tabelle 4.3: $\quad$ FADN-Stichprobe und Durchschnittsgewicht (Rechnungsjahr 1997, LBS 1997, StDB 1994)

\begin{tabular}{lccc}
\hline Land & $\begin{array}{c}\text { Tatsächlicher } \\
\text { Stichprobenumfang }\end{array}$ & $\begin{array}{c}\text { Anzahl der im } \\
\text { Beobachtungsfeld } \\
\text { vertretenen Betriebe }\end{array}$ & $\begin{array}{c}\text { Durchschnitts- } \\
\text { gewicht eines } \\
\text { Stichprobenbetriebes }\end{array}$ \\
\hline Belgien & 1.196 & 44.582 & 37 \\
Dänemark & 2.117 & 53.292 & 25 \\
Deutschland & 5.827 & 291.492 & 50 \\
Griechenland & 4.834 & 483.271 & 100 \\
Spanien & 8.221 & 537.325 & 65 \\
Frankreich & 7.568 & 406.804 & 54 \\
Irland & 1.202 & 128.006 & 106 \\
Italien & 16.235 & 913.974 & 56 \\
Luxemburg & 278 & 1.962 & 7 \\
Niederlande & 1.516 & 85.391 & 56 \\
Österreich & 2.085 & 90.242 & 43 \\
Portugal & 2.932 & 312.234 & 106 \\
Finnland & 1.007 & 48.480 & 48 \\
Schweden & 827 & 40.251 & 49 \\
Vereinigtes Königreich & 3.648 & 133.309 & 37 \\
\hline
\end{tabular}

Quelle: http://europa.int/comm/agriculture/rica/methodology2_de.cfm; zitiert am: 04.09.2007. 
Diese Unterschiede in den Auswahlsätzen zwischen den Mitgliedstaaten sind vor allem durch organisatorische und praktische Erhebungsschwierigkeiten bedingt, die naturgemäß in Ländern mit einem zahlenmäßig bedeutenden Agrarsektor vergleichsweise stärkeres Gewicht haben. Daher ist zu vermuten, dass nicht in jedem Fall ein optimaler Auswahlsatz in Bezug auf die Erfassung der Streuung bestimmter Untersuchungsmerkmale gefunden werden kann.

Um sicherzustellen, dass diese Stichprobe die Heterogenität der landwirtschaftlichen Tätigkeit angemessen widerspiegelt, wird von den Verbindungsstellen vor der Auswahl der Stichprobenbetriebe eine Schichtung des Beobachtungsfeldes vorgenommen. Dabei stützen sie sich auf die drei Kriterien Gebiet, wirtschaftliche Betriebsgröße und betriebswirtschaftliche Ausrichtung.

Die Dreiwegschichtung der Untersuchungsgesamtheit erlaubt deren Darstellung als dreidimensionale Zellenmatrix. Die Anzahl der landwirtschaftlichen Betriebe pro Zelle stützt sich auf die landwirtschaftliche Betriebsstrukturerhebung von EUROSTAT, die - wie oben erwähnt - die gleiche Klassifizierung wie die FADN-Erhebung verwendet.

Jede Zelle entspricht demzufolge einer spezifischen Betriebskategorie und Größenklasse und repräsentiert eine unterschiedliche Anzahl von landwirtschaftlichen Betrieben. Stichprobenbetriebe werden nicht aus dem Beobachtungsfeld (durch Testbetriebe repräsentierter Teil der Grundgesamtheit) als ganzes, sondern aus den Zellen des Beobachtungsfeldes ausgewählt, um die Heterogenität der FADN-Stichprobe im Beobachtungsfeld widerzuspiegeln. Abhängig davon, ob den Verbindungsstellen in den Mitgliedstaaten genügend Daten über die Variabilität der Betriebe innerhalb des Beobachtungsfeldes vorliegen, differieren die Auswahlsätze in den einzelnen Zellen. In Fällen, wo die Datenbasis nicht ausreicht, können keine optimalen Auswahlsätze errechnet, sondern nur die Anzahl der Betriebe in der betreffenden Zelle festgesetzt werden.

Die jährliche Stichprobe umfasst etwa 60.000 Betriebe (EU-15 ${ }^{12}$ ), davon ca. 4.500 spezialisierte Milchviehbetriebe. Die jährliche Stichprobe repräsentiert eine Gesamtzahl von etwa 4.000.000 landwirtschaftlichen Betrieben in den 15 Mitgliedstaaten, die über $90 \%$ der gesamten landwirtschaftlich genutzten Fläche (LF) verfügen und über $90 \%$ der gesamten landwirtschaftlichen Produktion der Union erwirtschaften.

Werden die 1997 gültigen Auswahlschwellen zugrunde gelegt, so ergeben sich die in der Tabelle 4.4 zusammengestellten Erfassungsbereiche für die Zahl der Betriebe und der landwirtschaftlichen Nutzfläche in der EU. Im Jahr 1997 repräsentierte die FADN- 
Stichprobe ca. 65 \% aller landwirtschaftlichen Betriebe (EU-15) und etwa 90 \% der landwirtschaftlichen Nutzfläche. Allerdings bestehen zwischen den Mitgliedstaaten hinsichtlich des Erfassungsbereichs deutliche Unterschiede. Das ist vor allem auf die unterschiedliche Bedeutung der Kleinbetriebe in den verschiedenen Mitgliedstaaten zurückzuführen. Wie oben schon erläutert, bleiben Kleinbetriebe, die zum Teil im Neben- oder Zuerwerb bewirtschaftet werden, bei der Auswahl unberücksichtigt. Daraus ergibt sich, dass die Repräsentativität generell in den mittleren Betriebsgrößen höher ist als in den ganz kleinen oder den ganz großen (KOMMISSION DER EUROPÄISCHEN GEMEINSCHAFTEN, 1982, S. 5).

Tabelle 4.4: Erfassungsbereich des FADN (LBS 1997/Jahr der landwirtschaftlichen Betriebsstrukturerhebung)

\begin{tabular}{|c|c|c|c|c|}
\hline \multirow[t]{2}{*}{ Mitgliedstaaten } & \multicolumn{2}{|c|}{ Betriebe ESU } & \multicolumn{2}{|c|}{$\begin{array}{c}\text { FADN- Erfassungsbereich } \\
\text { des Beobachtungsfeldes }\end{array}$} \\
\hline & Gesamtmenge & FADN Feld & Betriebe \% & LF \% \\
\hline Belgien & 67.180 & 45.345 & 67,5 & 93,5 \\
\hline Dänemark & 63.150 & 50.130 & 79,4 & 99,2 \\
\hline Deutschland & 536.060 & 304.400 & 56,8 & 93,3 \\
\hline Griechenland & 821.390 & 531.410 & 64,7 & 91,7 \\
\hline Spanien & 1.208 .260 & 776.780 & 64,3 & 88,6 \\
\hline Frankreich & 679.840 & 445.440 & 65,5 & 95,3 \\
\hline Irland & 147.830 & 129.910 & 87,9 & 96,4 \\
\hline Italien & 2.315 .230 & 1.242 .900 & 53,7 & 90,8 \\
\hline Luxemburg & 2.980 & 2.070 & 69,5 & 94,9 \\
\hline Niederlande & 107.920 & 84.060 & 77,9 & 94,1 \\
\hline Österreich & 210.110 & 88.050 & 41,9 & 62,1 \\
\hline Portugal & 416.690 & 327.420 & 78,6 & 95,7 \\
\hline Finnland & 91.440 & 59.640 & 65,2 & 84,6 \\
\hline Schweden & 89.580 & 42.440 & 47,4 & 83,8 \\
\hline Vereinigtes Königreich & 233.150 & 142.580 & 61,2 & 88,2 \\
\hline
\end{tabular}

1) Standartdeckungsbeitrag von 1994.

Quelle: http://europa.eu.int/comm/agriculture/rica/methodology2_de.cfm; zitiert am: 04.09.2007.

Die FADN-Datengrundlage ermöglicht die Selektion identischer Betriebe u. a. zur Darstellung von Betriebsentwicklungen, Berechnungen mehrjähriger Durchschnitte zur Nivellierung von Preis- und Ertragsschwankungen wie auch zur Gruppierung nach bestimmten Kriterien in Abhängigkeit von der Fragestellung. Sie finden im Rahmen von deskriptiven/statistischen Zeitreihen- und Querschnittsanalysen und für (positive und normative) Modellanalysen auf repräsentativer Datenbasis Verwendung. Das europäische Testbetriebsnetz stellt eine verlässliche Informationsquelle über regionalisierte und nach Betriebstypen differenzierte Einkommen landwirtschaftlicher Betriebe dar. 
Die Rechtsvorschriften beinhalten die Forderung, diese Daten landwirtschaftlicher Betriebe streng vertraulich zu behandeln. Es dürfen nur aggregierte Ergebnisse für eine Gruppe von Betrieben oder landwirtschaftlichen Betrieben in einem bestimmten Gebiet oder Mitgliedstaat bekannt gegeben werden.

Wegen dieser Durchschnittsbildung besteht die Gefahr, teilweise zu realitätsfernen Ergebnissen und Fehleinschätzungen bei der Analyse zu kommen. Aufgrund der anonymisierten Daten ist eine Rückkopplung zu den Landwirten - zu Kontrollzwecken oder bezogen auf das Anpassungsverhalten bei Politikänderungen - nicht möglich.

\section{Erneuerung der Stichprobe}

Auf der Grundlage des Informationsnetzes werden keine identischen Betriebe ausgewertet. Damit können zumindest bestimmte Minimalanforderungen hinsichtlich der Zufälligkeit der ausgewählten Betriebe erfüllt werden. Eine vollständige Zufallsauswahl ist jedoch aufgrund verschiedener Faktoren nicht zu erzielen:

- Verfügbarkeit einer landwirtschaftlichen Buchführung: Um einen FADN-Betriebsbogen auszufüllen, sollte eine geeignete Betriebsbuchführung (oder ähnliche finanzielle Informationen) zur Verfügung stehen. Einige Landwirte haben die notwendigen Informationen nicht verfügbar, so dass in diesen Fällen der Betriebsbogen nicht ausgefüllt werden kann. In mehreren Ländern helfen die Verbindungsstellen den Landwirten bei der Buchführung, wenn diese sonst nicht zustande käme. ${ }^{13}$

- Freiwillige Teilnahme: Die Teilnahme der Landwirte erfolgt auf freiwilliger Basis. Wenn einige der ursprünglich für die Stichprobe ausgewählten Betriebe auf ihre Teilnahme verzichten möchten, werden die Betriebe durch Betriebe aus derselben Zelle im Beobachtungsfeld ersetzt.

\section{Betriebsbogen}

Der Betriebsbogen stellt die Grundlage für die Erhebung und die Analysen des FADN dar. Durch die Ausgestaltung des Betriebsbogens ist sichergestellt, dass die verschiedenen Positionen nach einheitlichen Kriterien erfasst und alle zur Feststellung der Einkommen notwendigen Informationen übermittelt werden (Verordnung (EWG) Nr. 2237/77). Dieser Betriebsbogen ist allerdings nicht so detailliert wie der für die Zwecke des BMELVTestbetriebsnetzes verwendete, so dass nicht alle dort vorgenommenen Berechnungen auf EU-Ebene nachvollzogen werden können. Da viele Mitgliedstaaten schon vor der Schaf-

13 Insgesamt gesehen nimmt die Anzahl der buchführenden Betriebe kontinuierlich zu, bedingt u. a. durch die Bestimmung gemäß Verordnung (EWG) Nr. 797/85 des Rates vom 12.04.1985 (über die Modernisierung landwirtschaftlicher Betriebe). Danach erhalten Landwirte nur dann Investitionsbeihilfen, wenn sie eine landwirtschaftliche Buchführung aufweisen. 
fung eines europäischen Netzes bereits Erhebungen über die betriebswirtschaftlichen Verhältnisse/Betriebsführung durchgeführt haben, hatten sie ihre eigenen regionalen oder nationalen Betriebsbögen bereits entworfen. Diese liefern Daten, die zunächst den Mitgliedstaaten selbst dienen und erst in zweiter Linie zum Zwecke des FADN erfasst werden. Die Ziele der Mitgliedstaaten entsprechen nicht unbedingt mit jenen der Kommission überein. Die Betriebsbögen sind im Laufe der Zeit verbessert und an die Bedürfnisse der Anwender angepasst worden. Der FADN-Betriebsbogen ist im Hinblick auf die Tätigkeit des landwirtschaftlichen Betriebes enger gefasst als viele regionale und nationale Betriebsbögen.

Neben den Strukturdaten über die regionale Lage, die Besitzverhältnisse und die Arbeitskräfteausstattung werden die folgenden betrieblichen Daten erfasst:

- Bestand und Wert des Viehs,

- Viehzukäufe und -verkäufe,

- variable Aufwandspositionen,

- Boden- und Gebäudevermögen, Maschinen und Geräte sowie Umlaufvermögen,

- Verbindlichkeiten,

- Mehrwertsteuer,

- Prämien und Beihilfen,

- pflanzliche und tierische Produktion.

Theoretisch können über den Betriebsbogen etwa 2400 Einzelinformationen aufgenommen werden, die jedoch auf den Magnetbändern auf ca. 1.400 Informationen begrenzt werden. Im Durchschnitt der letzten Jahre enthielt jeder einzelne Betriebsbogen ca. 1000 Angaben zu den oben aufgelisteten Positionen.

Da die FADN-Datenbasis den steuerlichen Abschluss erfasst, fehlt ihm die für Politikfolgenabschätzungen erforderliche Datentiefe, insbesondere in Bezug auf das Mengengerüst ${ }^{14}$. Im Rahmen der Politikfolgenabschätzungen wird an zahlreichen Instituten in der EU mittels verschiedener Verfahren anhand von Plausibilitätsüberlegungen und externen In-

14 Aufgrund der Ermittlung der Einkommenslage der Landwirtschaft als Hauptzielrichtung erfolgt keine umfassende Zuordnung der Vorleistungen und der fixen Produktionsfaktoren auf einzelne Produktionszweige (vgl. VO (EWG) 2237/77, AB1 L 263). Eine Betriebszweigabrechnung, die in Mehrproduktbetrieben eine unabdingbare Voraussetzung für die Berechnung von Produktionskosten eines Produktes wäre, erübrigt sich jedoch in spezialisierten Betrieben weitgehend (ISERMEYER, 1988). Daraus folgt für diese Arbeit, dass hier weitgehend auf den Betriebszweig Milch spezialisierte Betriebe herangezogen werden. 
put/Output-Relationen versucht, Betriebszweigabrechnungen zu entwickeln. ${ }^{15}$ Diese sind jedoch EU-weit nicht vergleichbar.

\section{Datenqualität - Überprüfung der übermittelten Betriebsdaten}

Einerseits sind die Anweisungen zur Erstellung des verwendeten Betriebsbogens in der Regel eindeutig und für alle Mitglieder gleich, dennoch haben Erfahrungen gezeigt, dass es in Detailfragen zu Missverständnissen und Unstimmigkeiten kommen kann, die erst im Laufe der Auswertungen offensichtlich werden. Es werden Plausibilitätstests durchgeführt um zu vermeiden, dass auch nationale Umstellungen in der Datenerfassung zu gravierenden Veränderungen in den Auswertungen des FADN führen.

Die Verbindungsstellen verwenden ihre eigenen Kontrollverfahren, um eine Datenqualität sicherzustellen, die i. d. R. über dem von der Kommission geforderten Standard liegt. Wenn die Angaben fehlerfrei sind, tragen die Verbindungsstellen ihre nationalen Daten in den FADN-Betriebsbogen der Gemeinschaft ein, wie dies in der Verordnung (EWG) Nr. 2237/77 vom 23.09.1977 und in den nachfolgenden Änderungen ausgeführt ist. Danach übermitteln die Verbindungsstellen die Angaben nach Brüssel, wo sie in die von der Kommission durchgeführten Qualitätskontrollverfahren einbezogen werden.

Die Kommission wendet verschiedene Verfahren zur Sicherung der Datenqualität auf der Ebene des Einzelbetriebs an:

1. Beim ersten Verfahren handelt es sich um die Klassifizierung: Bevor Datenkontrollen zum Einsatz kommen können, werden alle landwirtschaftlichen Betriebe gemäß der Klassifizierung der Europäischen Union nach Wirtschaftsgröße und betriebswirtschaftlicher Ausrichtung eingestuft. Dabei können landwirtschaftliche Betriebe von der Zelle, in der sie ursprünglich eingeordnet waren, zu Auswahlzwecken in eine andere Zelle wandern. Dies kann z. B. dann der Fall sein, wenn sich die Größe eines Produktionszweigs zwischen dem Zeitpunkt der Stichprobenerfassung und dem Ende des Rechnungsjahres verändert hat.

2. Das zweite Verfahren betrifft die Durchführung von Plausibilitätstests. Es handelt sich dabei um Hunderte von Tests, die mögliche Fehler, widersprüchliche Daten und unwahrscheinliche Werte suchen und aufspüren sollen. Je nach Schwere des Fehlers werden verschiedene Fehlerklassen unterschieden. Die Fehler können unterschiedlicher Art sein (siehe Tabelle 4.5).

15

Als Beispiel sind hier die Studien von Pingault, Desbois (2003) und European Commission (2006) zu nennen. 
Fehler aufgrund unwahrscheinlicher Werte werden nach einem Wahrscheinlichkeitstest festgestellt, bei denen der Wert einer Variable, der im FADN-Betriebsbogen der Gemeinschaft angegeben wird, mit dem erwarteten Wert verglichen wird.

Das Datenkontrollsystem, das von der Kommission eingesetzt wird, ermöglicht die flexible Meldung von Ausnahmen. Es ermöglicht den Buchführungsexperten und Programmierern der Verbindungsstellen die Aufdeckung u. a. folgender Fehler:

- Fehler, die während der Übertragung des nationalen Betriebsbogens in den FADN-Betriebsbogen der Gemeinschaft entstanden sind,

- Fehler aufgrund der falschen Auslegung der Rubriken des FADN-Betriebsbogens der Gemeinschaft,

- $\quad$ Fehler und Auslassungen, die während der Datensammlung passiert sind.

Das System ermöglicht die Korrektur einzelner Betriebsbögen und erkennt auch jene Bögen, die zahlreiche Fehler aufweisen und daher aus der Stichprobe besser entfernt werden sollten.

Tabelle 4.5: $\quad$ Fehlerklassen

\begin{tabular}{ll}
\hline Art des Fehlers & Wahrscheinlicher Grund \\
\hline Codefehler & Fehler der Person, die die Codierung vornimmt \\
Rechenfehler & Fehler der Umrechnungsprogramme \\
Fehler aufgrund fehlender Angaben & Fehler des Büros, das den Betriebsbogen ausfüllt \\
Unwahrscheinliche Werte & \\
\hline
\end{tabular}

Quelle: Eigene Zusammenstellung (2007).

3. Mit Hilfe von Homogenitätstests wird eine Unterauswahl getroffen, die sich für besondere Analysen eignet. Sie stellen Ausreißer fest, also landwirtschaftliche Betriebe, bei denen sich der Wert einer oder mehrerer Variablen wesentlich vom Durchschnittswert der Klasse unterscheidet, zu der der landwirtschaftliche Betrieb gehört. Dies kann auf einen Fehler im Zuge der Datensammlung oder der Datencodierung hindeuten, der mit den Standardtests nicht entdeckt wurde.

4. Nachdem die Kontrollverfahren für die Daten einzelner landwirtschaftlicher Betriebe angewendet worden sind, beginnen die Kontrollverfahren auf Gesamtebene. Hierfür werden die Daten gewichtet und ausgehend von dem Gebiet, dem Mitgliedstaat, der 
Größenklasse und der betriebswirtschaftlichen Ausrichtung aggregiert. Anschließend erfolgen Kontinuitätstests. Dabei werden die errechneten Durchschnittswerte der Standardvariablen mit den erwarteten Durchschnittswerten verglichen, d. h. jenen Durchschnittswerten, die aufgrund früherer Trends erwartet würden.

5. Mit Hilfe von Kontinuitätstests wird anschließend die Prozentabweichung zwischen dem durchschnittlichen vorausgesagten Wert und dem beobachteten Wert errechnet. Wenn dieser Wert eine im Voraus festgelegte Schwelle überschreitet, produziert das Computerprogramm eine Meldung, aus der sowohl die Prozentabweichung als auch die absolute Abweichung hervorgeht. Die Daten werden daraufhin geprüft, ob es eine logische Erklärung dafür gibt. Ist dies nicht der Fall, werden die Daten auf betrieblicher Ebene korrigiert.

6. Das Korrekturverfahren wiederholt sich während der oben beschriebenen Qualitätsverfahren. Die Betriebsbögen, die der Korrektur bedürfen, werden von den Verbindungsstellen bearbeitet und müssen gegebenenfalls auf die Gebietsebene oder an die ursprüngliche Buchstelle zurückverwiesen werden. Teilweise werden die Betriebsbögen auch einfach ersetzt, weil die nationalen Stichproben in einigen Fällen größer sind als diejenigen, die von der Kommission zu FADN-Zwecken benötigt werden. Für die nationale Verbindungsstelle ist es leichter, einen Betriebsbogen durch einen anderen aus der eigenen Stichprobe des Mitgliedstaates zu ersetzen, als einen fehlerhaften Betriebsbogen zu korrigieren.

Wenn die Daten als fehlerfrei anerkannt werden, erfolgen die Berechnungen der betrieblichen Einzelpositionen, Analysen und Voraussagen mit den zuvor überprüften Betriebsdaten. Im Gegensatz zum Jahresabschluss des Bundesministeriums werden auf europäischer Ebene keine Jahresbilanz erstellt und keine Gewinn-und-Verlust-Rechnung durchgeführt. Vielmehr basiert die FADN-Auswertung auf der Einkommensrechnung für den landwirtschaftlichen Betrieb. Neben den auf den Magnetbändern aufgelisteten 700 betrieblichen Einzelpositionen werden von der Kommission bis zu 250 zusätzliche, abgeleitete Kennzahlen berechnet. Für jeden Betrieb liegen somit ca. 1.000 Positionen vor, was für jedes Rechnungsjahr mit etwa 60.000 Betrieben ca. 60 Millionen Einzelpositionen ergibt.

Diese sogenannten Standardergebnisse sind demzufolge auf Grundlage der Betriebsbögen errechnete statistische Daten, die von der Kommission periodisch erstellt und veröffentlicht werden. Sie liefern eine ausführliche Beschreibung der finanziellen Verhältnisse der Landwirte in der EU nach unterschiedlichen Gruppen. Die Standardergebnisse sind in einer öffentlichen Datenbank verfügbar. Die Kommission hat jede Variable in den Standardergebnissen definiert und damit versucht, eine weitgehende Übereinstimmung zwischen den Definitionen der eigenen Variablen und der Variablen anderer Organisationen bzw. landwirtschaftlicher Statistiken herzustellen. 
Zur möglichst sachgerechten Darstellung der Einkommenslage hat die Kommission Einkommensindikatoren verwendet, die ohne kalkulatorische Ansätze auskommen und die aus dem verwendeten Betriebsbogen möglichst direkt abgeleitet werden können. Bei den FADN-Standardergebnissen werden somit keine gesonderten Schätzungen der Entlohnung der familieneigenen Faktoren (kalkulatorischer Ansatz für Arbeit, Boden bzw. Kapital der Familie) vorgenommen.

Alle Ergebnisse werden mittlerweile in Euro angegeben. So können die Ergebnisse der einzelnen Mitgliedstaaten auf der Ebene der Europäischen Union aggregiert werden und die Ergebnisse zweier oder mehrerer Mitgliedstaaten verglichen werden.

Bis zum Abschluss aller für die Berechnung der Standardergebnisse nötigen Verfahren sollen ab dem Ende eines Rechnungsjahres nicht mehr als 15 Monate vergehen. Einige nationale Verbindungsstellen haben es in der Praxis nicht immer geschafft, den festgelegten Zeitplan einzuhalten. Folglich haben sich bei der Veröffentlichung der Gesamtgrößen der Europäischen Union als ganzes beträchtliche Verzögerungen ergeben. So kommt es, dass die Auswertungen des FADN den Mitgliedstaaten erst mit einer zeitlichen Verzögerung von ca. zwei Jahren zur Verfügung stehen. Ferner ist in einigen Mitgliedstaaten der Beginn des Rechnungsjahres nicht für alle landwirtschaftlichen Betriebe gleich. Dies führt dazu, dass sich der Verbuchungszeitraum für den Mitgliedstaat als Ganzes auf über zwölf Monate erstreckt. ${ }^{16}$ In der zeitlichen Verzögerung, mit der die Auswertungen zur Verfügung stehen, liegt ein Nachteil für die Verwendung von Buchführungsergebnissen als politische Entscheidungshilfe. Zur Vorbereitung von agrarpolitischen Entscheidungen werden nämlich Informationen über das laufende Rechnungsjahr benötigt, die Aussagen und ggf. auch Voraussagen über die Einkommensentwicklungen in den folgenden Rechnungsjahren ermöglichen.

\section{Hochrechnung}

Für die Berechnung der FADN-Ergebnisse wird ein besonderes Gewichtungssystem verwendet. Dieses Gewichtungssystem stützt sich auf das Prinzip der freien Hochrechnung. Hierbei erhält jeder Stichprobenbetrieb einen Gewichtungsfaktor, der der Anzahl der von ihm vertretenen landwirtschaftlichen Betriebe entspricht. Es wird wie folgt vorgegangen: Zunächst werden die Betriebe sowohl in der Stichprobe als auch im Beobachtungsfeld nach den folgenden drei Kriterien geschichtet: FADN-Region, betriebswirtschaftliche Ausrichtung und wirtschaftliche Betriebsgröße. Der Hochrechnungsfaktor ergibt sich aus

16

In Frankreich beginnt beispielsweise das Rechnungsjahr einer geringen Zahl von Betrieben zwischen dem 1. Oktober und dem 31. Dezember des Vorjahres (Steuerbuchführungen). Im Vereinigten Königreich erstreckt sich das Rechnungsjahr vom 31. Dezember bis zum 30. April. In Dänemark dauert für die Hälfte der Agrarbetriebe das Rechnungsjahr vom 1 .Januar bis zum 31. Dezember, bei den übrigen beginnt es am 1. April, 1. Mai oder 1. Juni und endet 12 Monate später. 
der Zahl der in einer Zelle des Beobachtungsfeldes vorhandenen Testbetriebe geteilt durch die Zahl der in der Stichprobe befindlichen Betriebe aus dieser Zelle.

Mit diesem Gewichtungssystem können verschiedene Auswahlsätze für verschiedene Zellen berücksichtigt werden. Für die Darstellung der FADN-Ergebnisse werden gewichtete Durchschnitte errechnet, indem die individuellen Gewichte auf die einzelnen Betriebe in der Stichprobe angewendet werden. Für die Errechnung der Gewichtungsfaktoren sind genaue und aktuelle Daten über das Beobachtungsfeld Voraussetzung. Diese werden aus der landwirtschaftlichen Strukturerhebung von EUROSTAT abgeleitet.

In Abbildung 4.2 ist das Klassifizierungsschema zur Berechnung der Hochrechnungsfaktoren dargestellt.

Abbildung 4.2: $\quad$ Klassifizierungsschema für die Berechnung der Hochrechnungsfaktoren

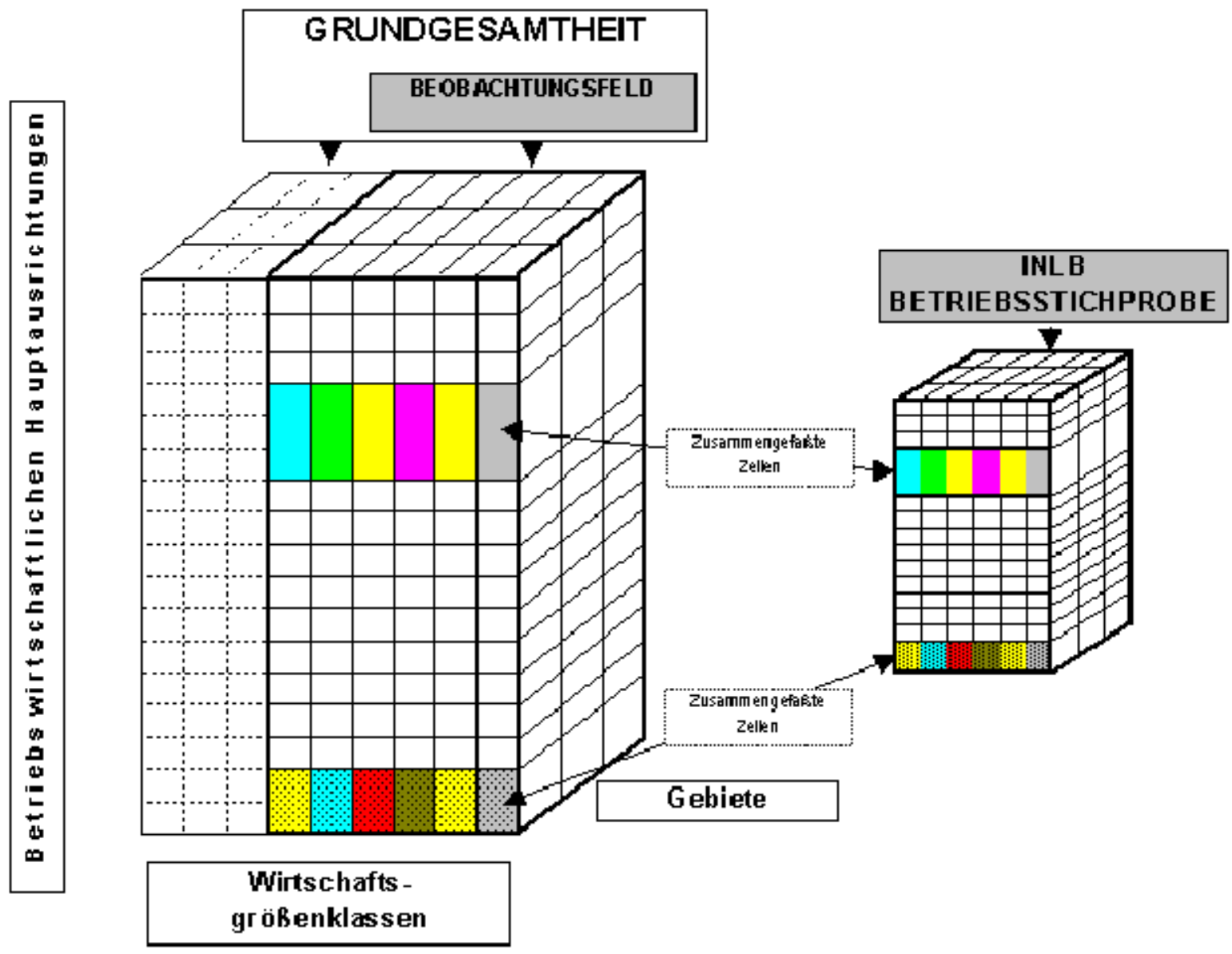

Quelle: http:/lec.europa.ew/agricul ture/rica/images/universe_de.gif; zitiert am: 04.09.2007. 


\subsection{Konzept des International Farm Comparison Network (IFCN)}

Das einzelbetriebliche IFCN-Konzept wurde für das Vorhaben dieser Arbeit ausgewählt, weil es nicht nur EU-weit, sondern auch in weiteren Ländern international harmonisiert angewendet wird und zudem eine hohe Datentiefe aufweist. Die gesamtbetrieblichen Daten liegen prozessspezifisch vor und sind getrennt nach Mengen und Preisen ausgewiesen. Diese Datentiefe ist in den amtlichen Buchführungsstatistiken (vgl. Kapitel 4.1) nicht zu finden. Im Folgenden werden die Zielsetzung und Entwicklung des IFCN sowie das Konzept und das Auswahlverfahren typischer IFCN-Betriebe, die Datenbasis und der Panelprozess ausführlich vorgestellt.

\subsubsection{Entwicklung und derzeitiger Stand}

Das Konzept „Representative Farms“ zur Politikfolgenanalyse wurde Mitte der 80er Jahre am Agricultural and Food Policy Center der Texas A\&M University (AFPC) mit dem Ziel entwickelt, die einzelbetrieblichen Auswirkungen von Politikvorschlägen zu analysieren. Die Basis bildeten ein Netzwerk von typischen Betrieben („Representative Farms“) und das Simulationsmodell FLIPSIM. ${ }^{17}$

Dieser „Representative-Farm-Ansatz“ basierte auf folgenden vier Elementen:

- Netzwerk typischer Betriebe: Netzwerk von ca. 90 regional typischen Betrieben der verschiedenen Produktionsrichtungen Futterbau, Marktfruchtbau, Schweinehaltung und Rindermast in den USA. Mit einem Berater, ca. vier bis sechs Landwirten und einem „FLIPSIM-Wissenschaftler“ werden sogenannte „typische Betriebe“ konstruiert. Gemeinsam werden die Eingabedaten für einen typischen Betrieb entwickelt und die Plausibilität der Analyseergebnisse überprüft.

- Modell FLIPSIM: ein zehnjähriges, einzelbetriebliches, dynamisches und stochastisches Simulationsmodell, welches flexibel konzipiert wurde, um alle aktuellen und zukünftigen Politikvarianten für verschiedene Betriebstypen abbilden zu können.

- Preisprojektionen: die Sektormodelle des FAPRI (Food and Agricultural Policy Research Institute, Iowa State University/University of Missouri-Columbia) liefern die Preisprojektionen für die Politikszenarien.

- Wissenschaftler des AFPC: ihre Aufgabe ist es, das Modell und die typischen Betriebe ständig zu aktualisieren. Im Diskussionsprozess mit den politischen Entscheidungsträ-

17 Detailliertere Modellbeschreibungen sind bei RICHARDSON and NIXON (1986) nachzulesen, siehe auch Kapitel 3 dieser Arbeit. 
gern werden Politikszenarien spezifiziert und zum Teil innerhalb weniger Wochen analysiert.

Basierend auf diesem Konzept wurde 1997 das International Farm Comparison Network (IFCN) am Institut für Betriebswirtschaft der Bundesforschungsanstalt für Landwirtschaft in Braunschweig (FAL) entwickelt (HEMme, 2000; DeBLITZ, 1998). Zunächst wurden die Branchen Milch, Rindfleisch und Ackerbau unter der Führung der FAL gemeinsam an der FAL entwickelt. Die Milchbranche wurde 1999 in eine „Public Private Partnership“ überführt. Das internationale Netzwerk typischer Milchviehbetriebe wird seit 2006 ausschließlich von einer Privatfirma koordiniert (www.ifcnnetwork.org), während das Institut für Betriebswirtschaft (FAL Braunschweig, seit 2008 vTI $^{18}$ ) in Kooperation mit der DLG den Rest des früheren IFCN unter dem Namen agri benchmark im Rahmen internationaler Verbände weiterentwickelt (www.agribenchmark.org).

Das IFCN-/agri benchmark-Konzept besteht aus folgenden Elementen:

- Das internationale Netzwerk der teilnehmenden Forschungsinstitutionen, Berater/Experten (Produktion, Vermarktung, Handel) und Landwirte, die eine nachhaltige Zusammenarbeit nach klar definierten Regeln aufbauen.

- Das einzelbetriebliche, dynamische Simulationsmodell TIPI-CAL.

- Der sogenannte „Panelprozess“ und das Konzept typischer Betriebe.

Das Grundprinzip von IFCN und agri benchmark ${ }^{19}$ ist identisch. IFCN beinhaltet das weltweite Netzwerk typischer Milchviehbetriebe, während agri benchmark die weltweiten Netzwerke typischer Rindermast- und Mutterkuh- sowie Ackerbaubetriebe führt. Im Bereich Milch arbeitet Agribenchmark eng mit dem Netzwerk der „European Dairy Farmers“ ${ }^{20}$ (EDF) zusammen.

Da zu Beginn dieser Arbeit das agri benchmark-Netzwerk“ noch nicht existierte und der Schwerpunkt der Analyse auf Milchproduktion liegt, beziehen sich die folgenden Aussagen lediglich auf das IFCN.

18

19

20

Johann Heinrich von Thünen-Institut.

Detaillierte Informationen sind auf der Internetseite des agri benchmark (www.agribenchmark.org) nachzulesen.

Das EDF-Netzwerk ermöglicht einen unmittelbaren Vergleich des eigenen Betriebes mit ausländischen Wettbewerbern. Die in diesem Netzwerk mitwirkenden Milchviehbetriebe haben u. a. die Möglichkeit ihre Daten in den jährlichen EDF-Kostenvergleich einzubringen. Nähere Informationen zum EDFNetzwerk sind nachzulesen bei REIL et al. (2001). 


\subsubsection{Zielsetzung}

Das Ziel des IFCN-Netzwerkes bzw. von agri benchmark ist es, auf Basis von typischen Modellbetrieben einen Einblick in die Wettbewerbsfähigkeit der Betriebe einer Region oder eines Landes zu bekommen. Dies beinhaltet im Einzelnen:

- Aufbau und Pflege einer Infrastruktur für die weltweite, nachhaltige Analyse landwirtschaftlicher Produktionssysteme.

- Erleichterung der Kommunikation sowie des Daten- und Informationsaustausches zwischen Wissenschaftlern durch harmonisierte Methoden.

- Analyse und Prognose landwirtschaftlicher Betriebe in den teilnehmenden Staaten unter verschiedenen strukturellen, technologischen und politischen Rahmenbedingungen.

\subsubsection{Konzept typischer Betriebe}

Im Folgenden wird das Grundkonzept typischer Betriebe im Rahmen des IFCN vorgestellt. Es wird dabei auf die Grundelemente „Typischer Betrieb“, Datenbasis, Panelprozess, Auswahlverfahren, Analyseinstrumentarien und Betriebsstrategien näher eingegangen.

\section{Definition „Typischer Betrieb“}

Unter einem „typischen Betrieb“ werden Betriebe verstanden, die von den nationalen Experten für den jeweiligen Standort im Hinblick auf Faktorausstattung, Faktor- und Produktpreise, Produktionssystem und Faktorproduktivitäten als typisch eingestuft werden.

Dieser typische Betrieb reflektiert einen bestimmten Anteil von ähnlich organisierten und strukturierten Betrieben. Die exakte 1:1-Verwendung der Daten real existierender Betriebe erfolgt im IFCN nicht. Daher besteht keine Datenschutzproblematik bei späterer Verwendung dieser Daten für wissenschaftliche Analysen.

\section{Auswahl der typischen Betriebe}

Bei der Auswahl typischer Betriebe wird im ersten Schritt ermittelt, welche Standorte eines Landes für ein bestimmtes Agrarprodukt (z. B. Milch, Rindfleisch, Getreide) besonders wichtig sind. Dies sind in der Regel die Produktionszentren, teilweise aber auch Standorte, an denen künftig eine besonders starke Produktionsausdehnung erfolgen könnte. Im zweiten Schritt werden Fachleute gesucht, die sowohl die Verhältnisse vor Ort gut kennen, als auch Zugriff auf regionale Buchführungsdaten haben und über einen intensiven Praxiskontakt verfügen. Mit diesen Personen, z. B. Spezialberatern, werden die wichtigsten Strukturmerkmale der zu bildenden Betriebe erörtert (z. B. Betriebsgröße, Betriebstyp). Wissenschaftler und Berater definieren gemeinsam die Eckpunkte für ein bis drei typische Betriebe pro Region. 
Anschließend werden die gebildeten typischen Betriebe nochmals mit den Daten aus der Grundgesamtheit und/oder mit Stichprobendaten abgeglichen („,crosscheck“). Im darauffolgenden Schritt werden die Betriebsleiter kontaktiert, deren Betriebe dem festgesetzten typischen Betrieb ähneln. Diese werden in den sogenannten Panel-Prozess (s. u.) einbezogen

Ziel ist es, für den gewählten Standort je einen durchschnittlich großen Betrieb zu bilden, um einen möglichst großen Anteil Betriebe und der Produktion aus der Grundgesamtheit widerzuspiegeln. Zudem wird ein größerer Betrieb gewählt, der in der Regel einen Wachstumsbetrieb darstellt und damit die Potenziale des jeweiligen Landes im Rahmen des internationalen Wettbewerbvergleichs veranschaulichen soll ${ }^{21}$. Bei der Auswahl typischer Betriebe werden folgende Kriterien zugrunde gelegt:

- Die typischen Betriebe sollten weniger als 50 \% außerbetriebliches Einkommen erzielen und/oder zumindest die Lebenshaltungskosten für eine Person decken.

- Der mittelgroße und größere typische Betrieb sollte ein durchschnittliches Management aufweisen (durchschnittliches Gewinnniveau).

Es werden Regionalstatistiken zur Betriebsgrößenverteilung zugrunde gelegt, um die Position der typischen Betriebe in der Verteilung der Grundgesamtheit bzw. der repräsentativen Stichproben festzustellen.

\section{Datenbasis für den typischen Betrieb/„Panel-Prozess“}

Für die Datenbasis des IFCN werden statistische Daten und Normdaten mit dem Expertenwissen landwirtschaftlicher Unternehmer und Berater vor Ort verknüpft. Ziel ist es, den typischen Betrieb möglichst detailliert und praxisgerecht zu erfassen. Dabei sollen alle relevanten Aspekte von der Organisation einzelner Betriebszweige bis zur Versteuerung des Gewinns berücksichtigt werden. Einfache Durchschnittsbetriebe, wie sie bei der Berechnung statistischer Durchschnitte für Betriebsgruppen entstehen, sollen vermieden werden. Die Grundlage für die Konkretisierung der typischen Betriebe ist:

- das international harmonisierte Datenerfassungssystem des IFCN,

- die Betriebszweigabrechnungen und die Buchführungsdaten der beteiligten Landwirte,

- $\quad$ ergänzende Unterlagen der regionalen Experten [z. B. Betriebszweigvergleiche, Buchführungsstatistiken, Normtabellen (vgl. auch PETER, 1994) und z. B. Daten des KTBL],

- das fachspezifische Expertenwissen aus den sogenannten „Panels“.

${ }^{21}$ Im agri benchmark-Netzwerk erfolgt inzwischen für einige Regionen eine weitere Untergliederung dergestalt, dass für Großbetriebe jeweils ein Betrieb mit durchschnittlichem und ein Betrieb mit überdurchschnittlichem Management gebildet wird. 
Die einzelbetrieblichen Daten für die typischen Betriebe werden im sogenannten „PanelProzess“ („Expertenrunde“) mit einem Berater vor Ort und bis zu sechs Landwirten sowie einem IFCN-Wissenschaftler ermittelt.

Die Vorgehensweise basiert auf einem für alle Länder einheitlichen Standardfragebogen. In Abhängigkeit von der Fragestellung wird ein kleines ("Pre-Panel”) oder ein volles Panel ("Full Panel”) für jeden typischen Betrieb durchgeführt (siehe Abbildung 4.3). Im ersten Schritt tritt in der Regel nur ein „Pre-Panel“ zusammen, an dem lediglich der nationale Koordinator, der regionale Berater sowie ein ausgewählter Landwirt mitwirken.

Individuelle Betriebsdaten und „Pre-Panel“- Informationen reichen aus für internationale Status-quo-Analysen zu Erlösen, Kosten und Rentabilität (siehe HEMME et al., 2007, IFCN Dairy Report 2007, S. 14). „Full Panels“ werden gebildet, um betriebliche Anpassungsstrategien zu diskutieren und zu spezifizieren. Ein großer Vorteil der „Pre-Panels“ und der individuellen Datenerhebungen ist, dass sie im Vergleich zu offiziellen Statistiken sehr viel schneller zu organisieren sind.

In allen Fällen erfolgt nach Berechnung der erhobenen Daten eine Rückkopplung mit den beteiligten Landwirten und Beratern zur Kontrolle und Verbesserung der Ergebnisse. Dieser iterative Prozess wird so lange durchgeführt, bis plausible und realitätsnahe Ergebnisse für die typischen Betriebe vorliegen.

Abbildung 4.3 veranschaulicht zusammenfassend die beschriebene Datenerhebungsmethode, bei der statistische Daten und Normdaten mit dem Expertenwissen landwirtschaftlicher Unternehmer und Berater vor Ort verknüpft werden, um eine möglichst realitätsnahe, aktuelle Abbildung der physischen und monetären Abläufe einzelner Betriebe in verschiedenen Regionen der Welt zu erzielen.

Zur Simulation der typischen Betriebe werden sowohl Daten aus der Vergangenheit als auch Annahmen zur zukünftigen Entwicklung von Preisen, Erträgen und Kosten benötigt. Dafür ist es notwendig, den mikroökonomischen Ansatz von IFCN mit landwirtschaftlichen Sektor- und Handelsmodellen zu verbinden, die in der Lage sind, langfristige Projektionen verschiedener Variablen (u. a. Preise, Produktion und Konsum) zu entwickeln. Für die Simulation werden in der Regel folgende Datenquellen herangezogen:

- Projektionen für diverse Variablen, die in regelmäßigen Abständen von verschiedenen Institutionen veröffentlicht werden (z. B. USDA, OECD, FAPRI, Europäische Kommission).

- Schätzungen aus Konsortien (wie z. B. FAL-Modellverbund), welche hoch aggregierte Modelle verwenden, die weltweite Entwicklungen bezüglich Preise etc. projizieren können (z. B. GTAP).

- Ergebnisse von „Ad-hoc“-Expertenbefragungen zu bestimmten Fragestellungen. 
Abbildung 4.3: Datenerhebungsverfahren zur Erfassung typischer Betriebe

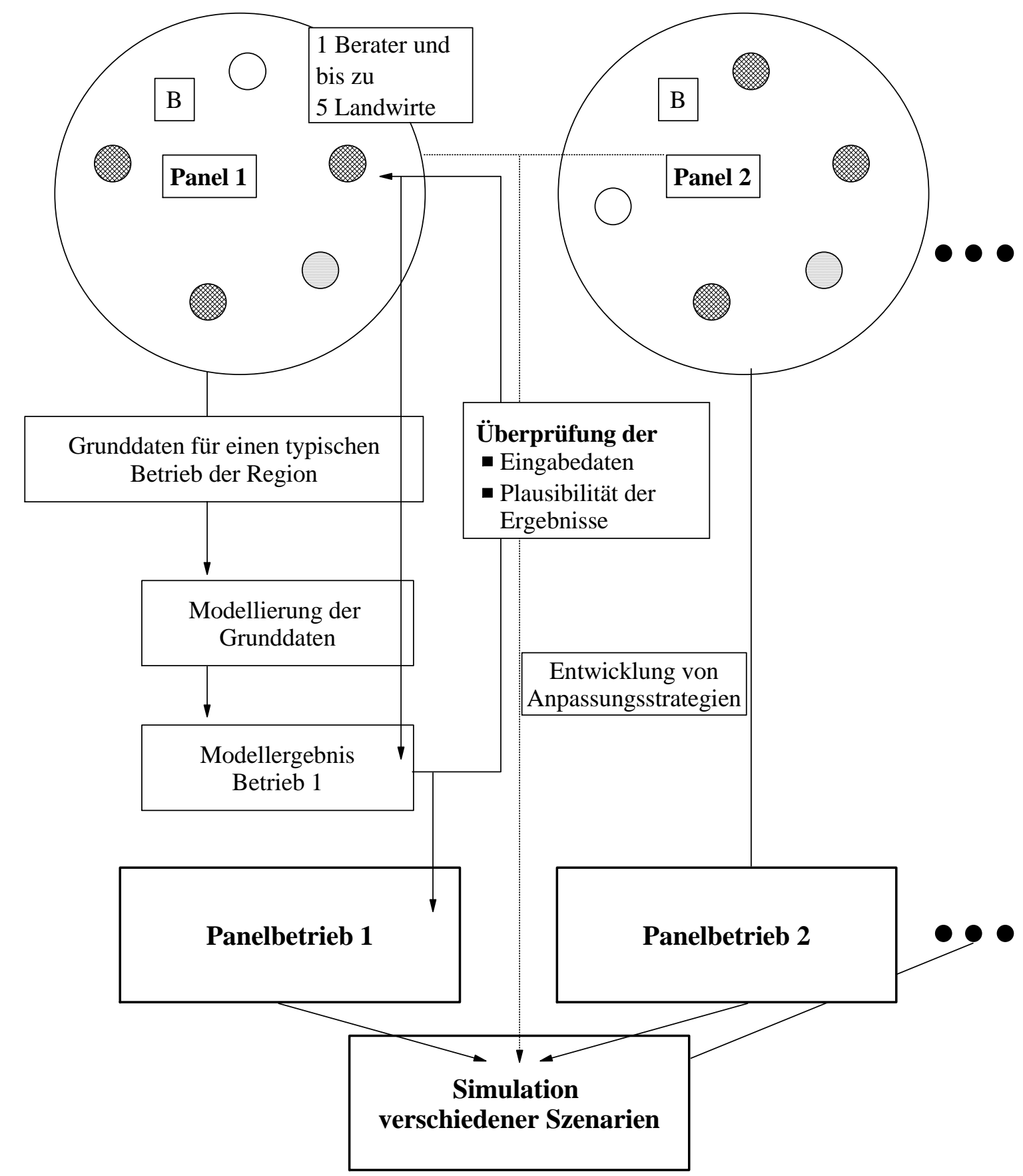

Quelle: TIPI-CAL, Hemme (1997). 


\section{Aktualisierung der Daten}

Im IFCN erfolgt eine regelmäßige Aktualisierung der typischen Betriebe. Prinzipiell ist eine jährliche Aktualisierung möglich, teilweise erfolgt auch ein Aktualisierung im Abstand von zwei bis drei Jahren, wobei in den Zeiträumen zwischen der Aktualisierung die Möglichkeit besteht, die Betriebe unter Verwendung des Simulationsmodells sowie nationaler Datenbanken fortzuschreiben.

Zu bestimmten Fragestellungen können ergänzende Erhebungen durchgeführt werden. Die Ergebnisse der Analysen (z. B. Kostenanalysen, Projektionsrechnungen) werden dem kritischen Urteil der Praktiker ausgesetzt und gegebenenfalls nachgebessert.

\section{Datenkontrolle}

Bei der Datenkontrolle werden im ersten Schritt die Ergebnisse (z. B. von Simulationsrechnungen betrieblichen Anpassungsverhaltens) mit ergänzenden statistischen Daten und Normdaten hinsichtlich ihrer Plausibilität überprüft. Im zweiten Schritt werden die Landwirte und der Berater mit dem Ergebnis konfrontiert, so dass Korrekturen erfolgen können. Das Ergebnis der abschließenden Simulation wird den Experten vor Ort präsentiert, und es erfolgt eine Bestätigung über die Plausibilität des gesamten Datengerüstes des typischen Betriebes der Region in der Ausgangssituation und für die genannten (Anpassungs)Strategien bei Änderung der (politischen) Rahmenbedingungen.

\section{Analyseinstrumentarien}

Für Betriebsentwicklungsstrategien und Politik- sowie Technikfolgenanalysen werden das Modellsystem TIPI-CAL und seine Unterstützungsmodule verwendet.

Mit den makrogesteuerten Unterstützungsmodulen lassen sich nach Spezifikation der Szenarien und Projektionen Politik- und Technologieszenarien oder Entwicklungsstrategien berechnen und ihre Auswirkungen auf ausgewählte Erfolgskennzahlen (z. B. Gewinn) darstellen. Eine Mischung von Szenarien und Strategien ist möglich.

In der Ausgangssituation wird der Betrieb bei der Festlegung des monetären und physischen Datengerüsts in einer bestimmten Entwicklungsphase erfasst. Von diesem Startpunkt aus kann er sich gemäß seiner betriebsindividuellen Strategie weiterentwickeln. Die Änderungen der politischen Rahmenbedingungen können je nach betriebsindividuellem Anpassungsspielraum möglicherweise verschiedene Anpassungen der Betriebsstrategie bewirken. Bei der einzelbetrieblichen dynamischen Politikfolgenanalyse werden für den einzelnen Betrieb vorab das sogenannte Referenzszenario und eine Strategie bei Änderung der politischen Rahmenbedingungen festgelegt. Bei Betrachtung mehrerer Betriebe ist darüber hinaus zu entscheiden, ob für alle Betriebe im Referenz- und/oder Politikszenario die gleiche Strategie festgelegt werden soll oder nicht. Die Analyse könnte sowohl für das 
Referenzszenario als auch für die Politikstrategien mit für jeden Betrieb unterschiedlichen Strategien erfolgen. Im Rahmen des Konzepts typischer Betriebe werden dabei die von den Landwirten genannten betrieblichen Strategien und Anpassungsstrategien im Modell berücksichtigt.

Bisher standen der Ausbau des Netzwerks und der Vergleich der Ist-Situation im Vordergrund. Es gibt bisher nur wenige Studien, in denen typische Betriebe für Politikfolgenanalysen simuliert werden.

\subsection{Schlussfolgerungen}

Der Überblick über die in der quantitativen Analyse zur EU-Milchmarktpolitik verwendeten Datengrundlagen verdeutlicht, dass der Aufwand zur Harmonisierung und Ergänzung der bisher nur regional oder national verfügbaren Datenbestände sehr hoch ist und weder innerhalb dieser Studie, noch innerhalb anderer Studien, die in der Regel recht kurzfristig für Zwecke der Politikberatung durchzuführen sind, zu leisten ist. Deshalb gilt es eine Infrastruktur zu schaffen, die international harmonisierte Daten für kurzfristige Analysen vorhält.

Für das Vorhaben dieser Arbeit sind - in Anlehnung an das in Kapitel 3 entwickelte Anforderungsprofil - zwei Datenquellen ausgewählt worden, die EU-weit harmonisiert erhoben werden und aufgrund ihrer Eigenschaften sich möglicherweise gegenseitig sinnvoll ergänzen können. Es handelt sich dabei um die Datensätze des FADN- und des IFCNNetzwerkes. Zusätzlich werden weitere der hier vorgestellten Datenquellen herangezogen, um die Ergebnisse aus den FADN- und IFCN-Berechnungen sowie aus der Verknüpfung beider Datensätze in den statistischen Rahmen einzuordnen und auf Plausibilität zu überprüfen sowie sinnvoll zu ergänzen.

Im folgenden Kapitel wird kurz erläutert, wie FADN und IFCN für Analysen zur Liberalisierung des EU-Milchmarktes eingesetzt worden sind. Dabei werden die Begrenzungen der jeweiligen Datensätze aufgezeigt und anschließend Schlussfolgerungen für eine kombinierte Anwendung gezogen. 


\section{Ermittlung der Potenziale von FADN- und IFCN-Daten anhand von ausgewählten Analyseergebnissen}

In diesem Kapitel wird erläutert, wie FADN und IFCN für Analysen zur Liberalisierung des EU-Milchmarktes bisher eingesetzt worden sind. Dazu werden zwei Analysekonzepte vorgestellt: a) der Einsatz für die Produktionskostenanalyse und b) der Einsatz für die modellgestützte Politikfolgenabschätzung. Dabei wird herausgearbeitet, wo mögliche Fehler der Ergebnisse bzw. Begrenzungen der Aussagekraft liegen. Auf dieser Basis werden abschließend Schlussfolgerungen für die kombinierte Anwendung von FADN- und IFCNDaten in der Politikanalyse gezogen.

\subsection{Einsatz von FADN- und IFCN-Daten für Produktionskostenanalysen}

Im Folgenden wird der Einsatz von FADN und IFCN bei der Produktionskostenanalyse erläutert. Dies erfolgt vor dem Hintergrund einer zunehmenden Liberalisierung des Milchmarktes, die eine Abwanderung der Milch zu „low cost regions“ erwarten lässt. Mit Hilfe der Produktionskosten können Produktionsverlagerungen abgeschätzt werden.

\subsubsection{Produktionskostenanalyse mit FADN-Daten}

Die folgenden Produktionskostenschätzungen auf Basis von FADN-Daten wurden im Rahmen eines EU-Projektes ${ }^{1}$ vorgenommen. Im Vordergrund der ökonomischen Auswertungen standen $\mathrm{u}$. a. folgende agrarpolitisch relevante Fragestellungen bezogen auf die Zwischenbewertung der Agenda 2000:

- An welchen Standorten befinden sich Betriebe in der EU (EU-15), die von einer Milchpreissenkung am stärksten betroffen sind? (ELPEN-Projekt, Zwischenbericht 2001, HINRICHS und JÄGERSBERG, 2001)

- Im Hinblick auf betriebliche Anpassungsreaktionen: In welchen Regionen und Betriebstypen ist eine Ausweitung bzw. Einschränkung der Milchproduktion zu erwarten? (ELPEN-Projekt, Zwischenbericht 2002, HINRICHS und JÄGERSBERG, 2002)

1 EU-Projekt ELPEN - European Livestock Policy Evaluation Network, Projektlaufzeit: 01.02.2000 bis 30.06.2003. 
Die Datengrundlage für die ökonomischen Auswertungen in dem expertengestützten System ELPEN(WRIGHT et al., 2003) ${ }^{2}$ bildeten im Wesentlichen die FADN-Daten (60.000 Stichprobenbetriebe in der EU-15) der Jahre 1996, 1997 und 1999. Um fehlende Daten zu berechnen, wurde im Rahmen des EU-Projektes ELPEN ${ }^{3}$ das Kostenallokationsmodell von GoERTz (1999) ergänzend herangezogen.

In dieser Arbeit wurden zur Klärung der oben genannten Fragen die Produktions-, Gewinn- und Liquiditätsschwelle zugrunde gelegt (vgl. DEEKEn und HEMme, 2002). Dabei wurde angenommen, dass die Milchproduktion eingestellt wird, wenn der Milchpreis unter die Produktionsschwelle ${ }^{4}$ sinkt. Die Liquiditätsschwelle ${ }^{5}$ wurde berechnet, um zu untersuchen, wie die Betriebe mittelfristig reagieren werden. Die Gewinnschwelle ${ }^{6}$ stellt den langfristig benötigten Milchpreis dar, um die gesamten Kosten im Betriebszweig Milch zu decken und eine Entlohnung für die familieneigenen Produktionsfaktoren zu erzielen. Die Nebenerlöse ${ }^{7}$ wurden jeweils abgezogen, um die drei Schwellen direkt auf den Milchpreis beziehen zu können.

Es wurde angenommen, dass ein (hoher) Überschuss des Milchpreises über die (langfristigen) Produktionskosten einen Anreiz zur Ausdehnung der Produktion darstellt, da er Kapital für mehr Investitionen schafft. Hierbei ist jedoch zu beachten, dass der Einkommensindikator ein Faktor unter mehreren Größen ist, die zu Anpassungsreaktionen des Landwirts führen.

Zunächst ist in der Karte 5.1 die Produktionskostenschätzung (Vollkosten) spezialisierter Milchviehbetriebe auf Basis von FADN-Daten aufgezeigt. Niedrige Produktionskosten sind insbesondere in einigen Regionen Frankreichs, Großbritanniens, in den Niederlanden und in Ostdeutschland zu verzeichnen. Die Karte 5.2 stellt auf FADN-Regionsebene ${ }^{8}$ die Differenz zwischen Milcherlösen und Vollkosten (je $100 \mathrm{~kg}$ Milch) im Wirtschaftsjahr 1999/2000 bei einer Milchpreissenkung von 20 \% (MTR-Beschluss Juni 2003) dar. Eine

2 EU-Projekt ELPEN - European Livestock Policy Evaluation Network, Projektlaufzeit: 01.02.2000 bis 30.06.2003.

3 EU-Projekt „ELPEN“ - European Livestock Policy Evaluation Network - Untersuchung der Auswirkungen agrarpolitischer Entwicklungen auf die tierische Produktion in der EU (Schwerpunkt: Milcherzeugung). Projektlaufzeit: 01.02.2000 bis 30.06.2003.

Milchpreis, zu dem variable Kosten gedeckt werden (Deckungsbeitrag $=0$ ).

$5 \quad$ Milchpreis, zu dem laufende Ausgaben gedeckt werden (Cashflow II = 0).

$6 \quad$ Milchpreis, zu dem Vollkosten gedeckt werden (Unternehmergewinn $=0$ ).

7 Zu den Nebenerlösen zählen: Rindfleischerlöse, Erlöse aus Kälber- und Färsenverkäufen und Direktzahlungen. Annahme: Nebenerlöse = Kosten ihrer Produktion.

8

Zur Definition der FADN-Regionen siehe Kapitel 3.2.3. 
besonders ungünstige Einkommenssituation der Milchviehbetriebe ist in Finnland, Schweden und Österreich zu verzeichnen. ${ }^{9}$ Bei einer Milchpreissenkung ist dort mit einer Verringerung der Milchproduktion zu rechnen. Als wenig betroffen bzw. als relativ „existenzgesichert“ werden Betriebe in Teilen Belgiens, Großbritanniens, Dänemark, Ostdeutschland sowie im Norden Spaniens und in Teilen Italiens ${ }^{10}$ eingestuft. In den zuletzt genannten Regionen ist den Annahmen zufolge betriebliches Wachstum als Anpassungsreaktion auf die Milchmarktreformen zu erwarten.

Karte 5.1: Vollkosten der Milchproduktion auf Basis von FADN-Daten in Euro/dt (Wirtschaftsjahr 1999/2000)

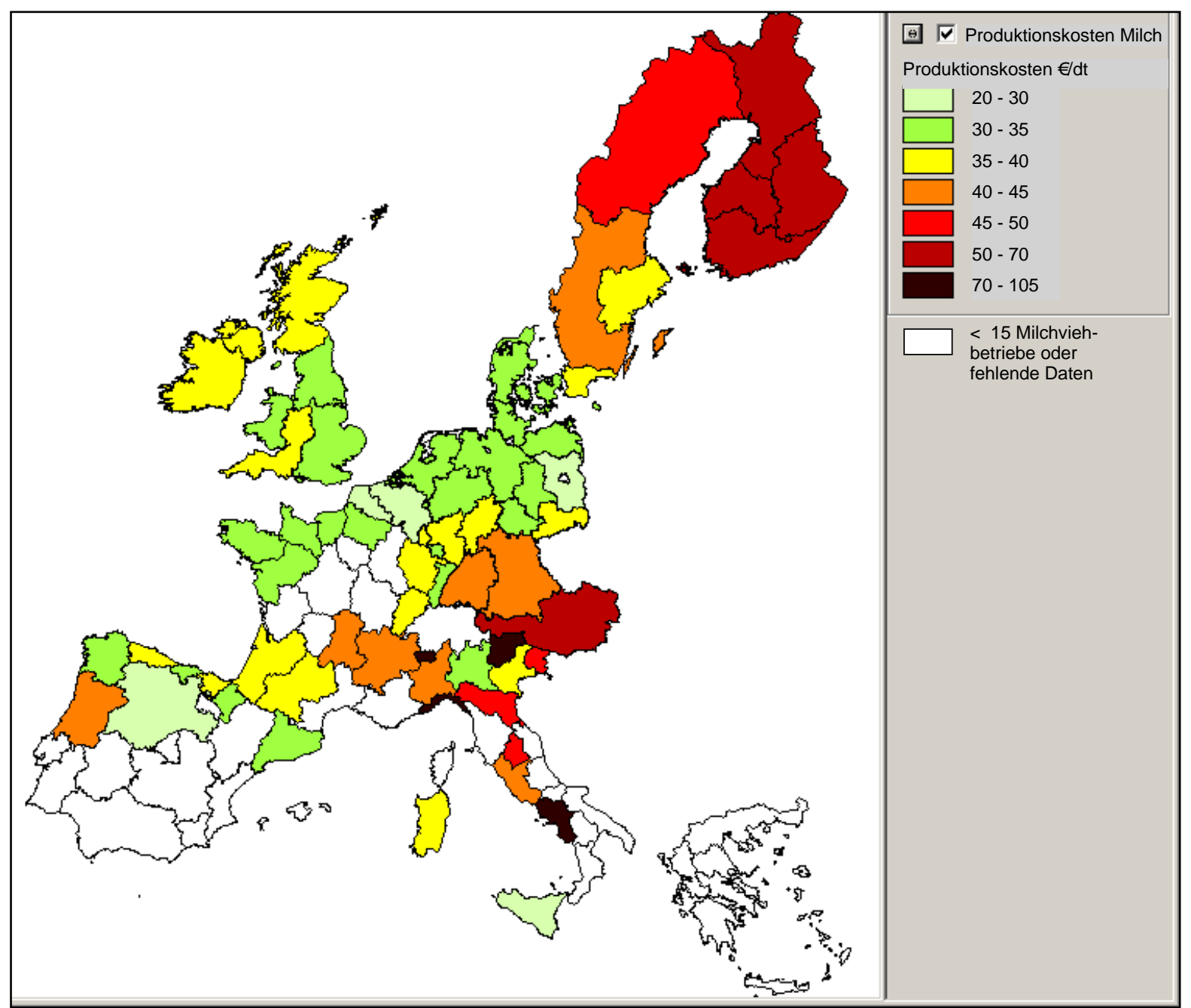

Quelle: Eigene Berechnung und Darstellung siehe ELPEN-System (2003).

9 In den weniger erfolgreichen Betrieben waren schon in der Ausgangssituation die Produktionskosten nicht durch den Milchpreis gedeckt.

10

Teilweise auch bedingt durch die hohen Milchpreise. 
Karte 5.2: Differenz zwischen Milcherlösen und den Vollkosten bei einer Milchpreissenkung um 20\%; Datenbasis: FADN-Daten (Wirtschaftsjahr 1999/2000)

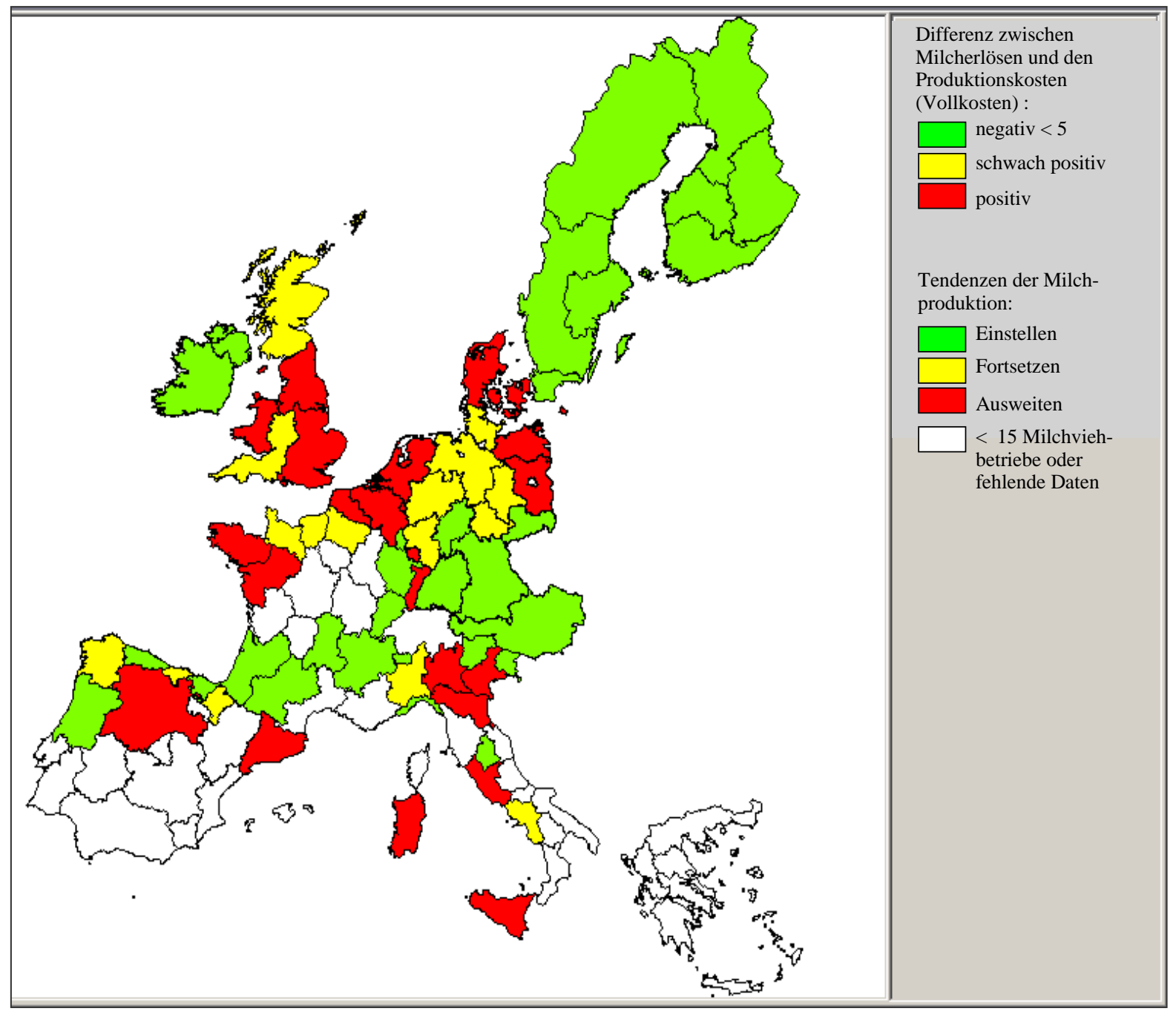

Quelle: Eigene Berechnung und Darstellung; siehe auch ELPEN-System (2003). 
Um die Einschätzungen zu untermauern ist es erforderlich, weitere Einflussfaktoren hinzuzuziehen. Die Ex-post-Betrachtung einer Änderung der Viehbestände und der Anzahl der Milchviehbetriebe im Zeitraum von 1990 bis 1999 in den berücksichtigten EURegionen liefert trotz bestehender Milchquotenregelung ${ }^{11}$ bereits zusätzliche Informationen bezüglich der Potenziale der Milchproduktion (siehe Anhang Karten A5.1 und A5.2). Es konnte nachgewiesen werden, dass in den Regionen, in denen betriebliches Wachstum prognostiziert worden ist, größtenteils schon in der Vergangenheit eine Erhöhung der Viehbestände und eine Verringerung der Anzahl der Milchviehbetriebe zu verzeichnen waren (siehe Anhang Karten A5.1 und A5.2).

Für die Erstellung der Karten 5.1 und 5.2 wurde für jeden Mitgliedstaat der EU jeweils ein Durchschnittsbetrieb kalkuliert. Eine Differenzierung in verschiedene Betriebsgrößenklassen erfolgte nicht. Eine aussagekräftige Analyse der Ursachen für die Kostenunterschiede ist nicht möglich, da eine Rückkopplung zu den Unternehmern nicht erfolgen kann.

Im Rahmen einer Studie von ISERMEYER et al. (2006) zu verschiedenen agrarpolitischen Handlungsoptionen auf dem Milchmarkt wurden Ergebnisse vorgestellt, die sich ebenfalls auf die ökonomische Verwundbarkeit spezialisierter Milchviehbetriebe bei verschiedenen Liberalisierungsszenarien beziehen sowie mögliche Produktionsausdehnungen in den EURegionen prognostizierten. Dabei wurde die Differenz von Milcherlösen und variablen Kosten bei sinkenden Milchpreisen in den EU-Regionen analysiert. Vergleicht man die oben dargestellten Ergebnisse aus dem EU-Projekt ELPEN mit den Darstellungen bei ISERMEYER (2006) in Abbildung 5.1, zeigt sich z. B. für den Osten Großbritanniens ein ähnlich positives Bild, während Österreich und Finnland deutlich besser abschneiden und insbesondere für Österreich deutliche Produktionssteigerungen prognostiziert werden. Diese Unterschiede in den Ergebnissen zeigen sehr anschaulich, dass die Datenlage und das vorhandene Instrumentarium zur Politikfolgenabschätzung verbesserungsbedürftig sind.

Eine besondere Stärke von FADN ist es, die Variabilität der Ergebnisse darzustellen. Dies wird in Abbildung 5.2 am Beispiel von Produktionskostenberechnungen spezialisierter Milchviehbetriebe in Frankreich verdeutlicht. In Abbildung 5.2 ist die kumulierte Verteilung der französischen Milchviehbetriebe, sortiert nach den Produktionskosten dargestellt. Anhand der Abbildung wird insbesondere die große Varianz in den Vollkosten deutlich, die sich im Bereich zwischen 25 bis zu $55 €$ bewegen.

Da der Milchquotenhandel innerhalb bestimmter Gebiete eingeschränkt erlaubt ist, sind intraregional dennoch Tendenzen über die relative Vorzüglichkeit der Milchproduktion einzelner Betriebstypen (siehe auch Kapitel 2.1/Entwicklung der Bestandsgrößenklassen) und Standorte ableitbar. 
Abbildung 5.1: $\quad$ Milchpreise, Produktionskosten und Gewinnspannen in spezialisierten Milchviehbetrieben der EU-15, 2003, in c/kg1)

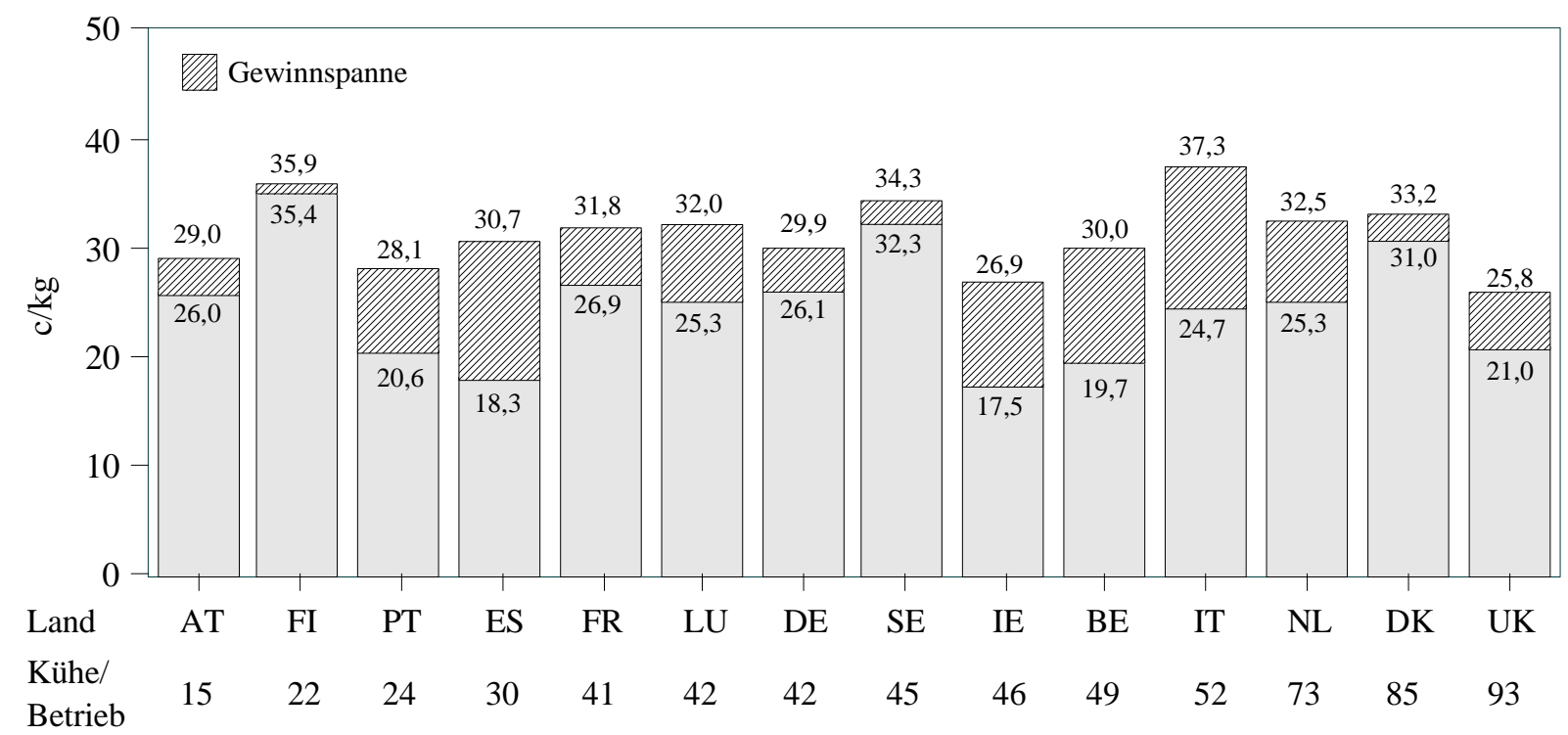

1) Ohne Kostanansätze für unternehmenseigene Produktionsfaktoren. Quelle: EU-Kommission, Auswertung des FADN.

Abbildung 5.2: $\quad$ Kumulierte Verteilung der Milchviehbetriebe, sortiert nach der Höhe der Vollkosten und der Vollkosten abzüglich kalkulatorischer Größen (Beispiel: Frankreich)

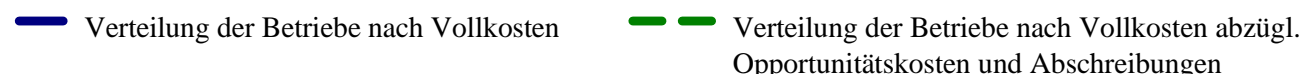

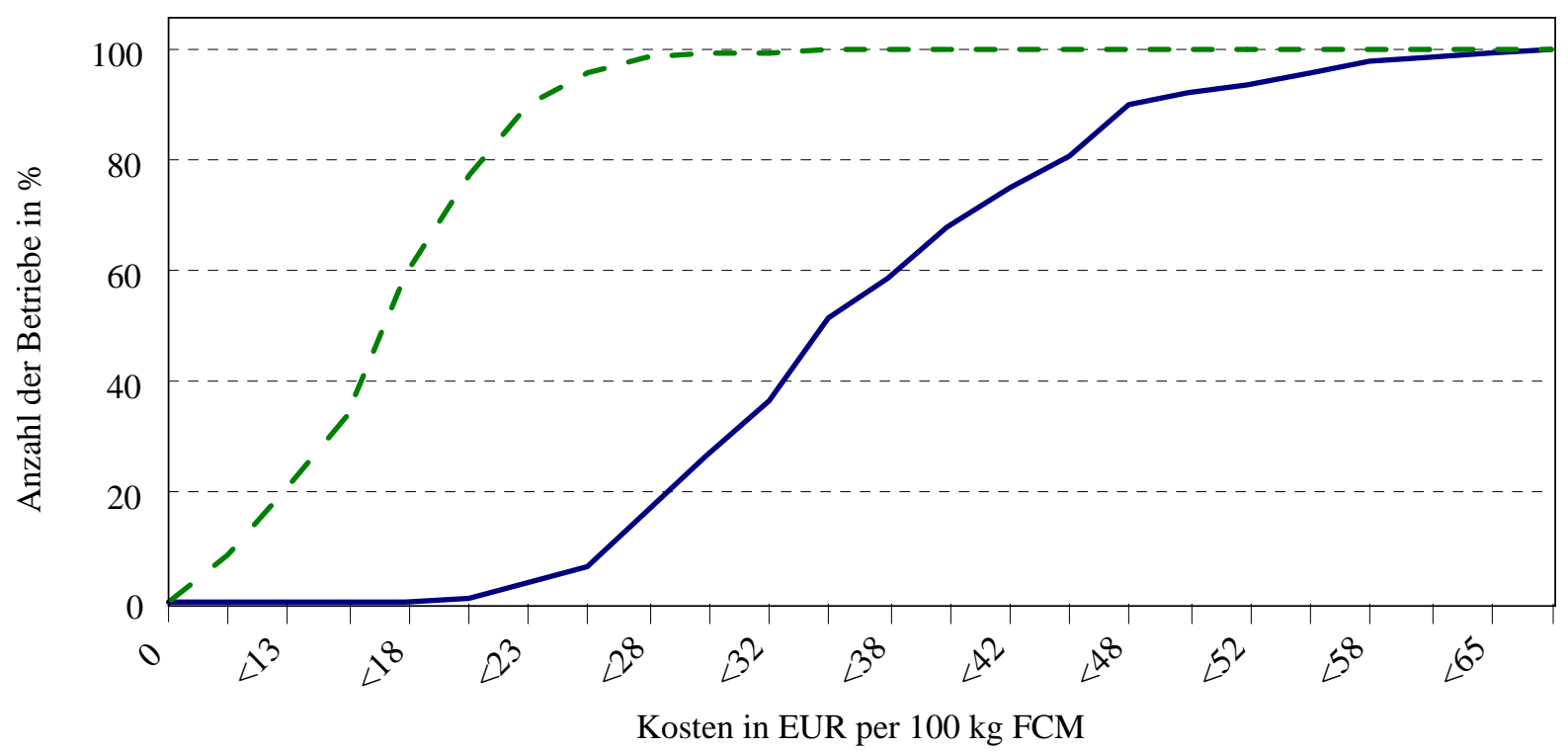

Quelle: Eigene Berechnung und Darstellung in PACIOLI 10, LEI, 2002. 
In Abbildung 5.3 wird die Variabilität der Produktionskosten in Abhängigkeit von der Milchleistung veranschaulicht. Zwischen den Milchleistungsklassen sind deutliche Unterschiede in den Vollkosten der Milcherzeugung festzustellen. Die Differenz der Produktionskosten beträgt zwischen oberem und unterem Viertel etwa 16 bis $23 € / 100 \mathrm{~kg}$ Milch.

Abbildung 5.3: Vollkosten der Milchproduktion abzüglich Nebenerlöse (Beispiel: Frankreich)
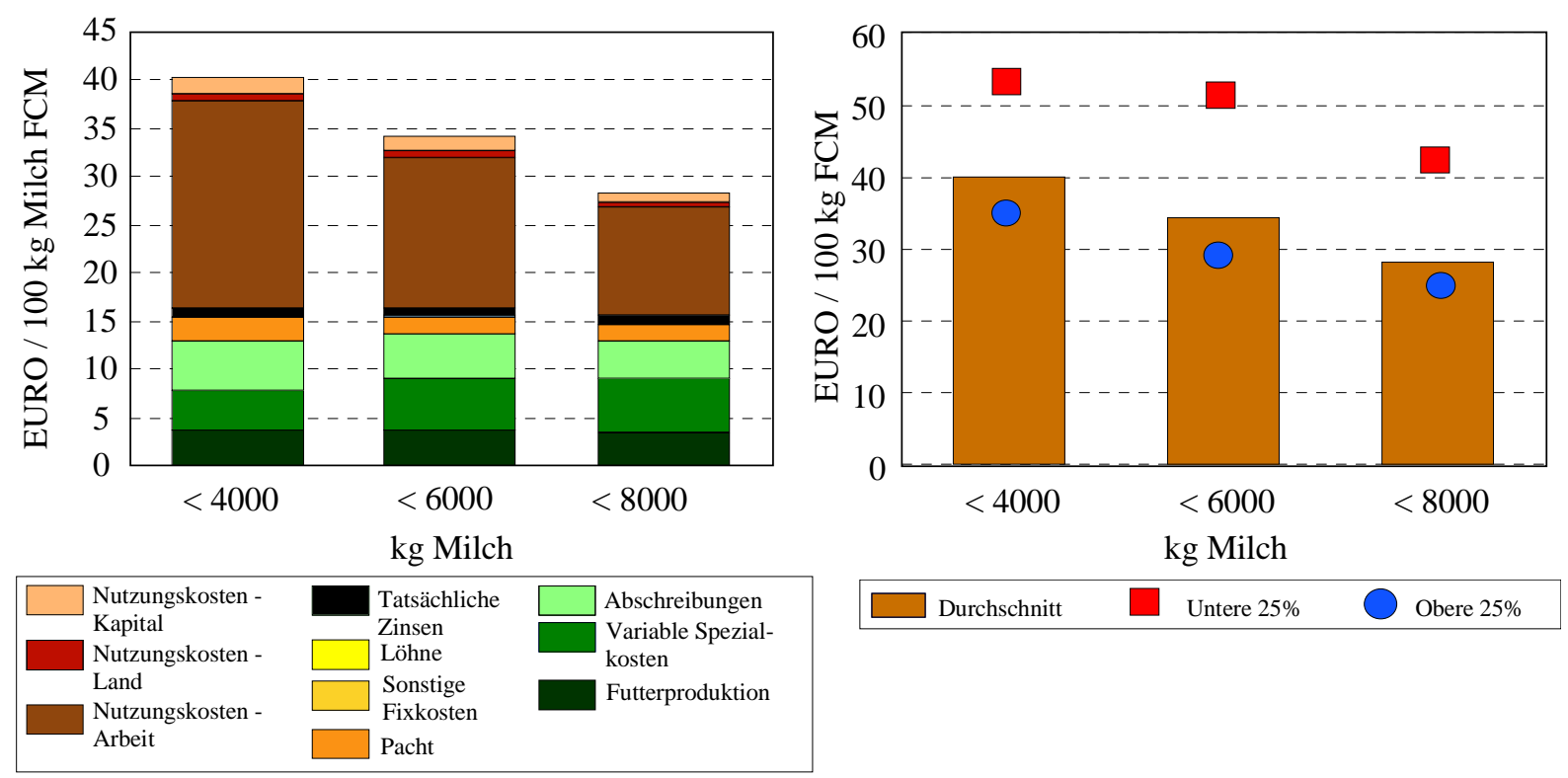

Quelle: Eigene Berechnung und Darstellung in PACIOLI 10, LEI, 2002.

Die Produktionskostenberechnungen von GoERTz (1999) mit deutschen Testbetriebsdaten in Abbildung 5.4 veranschaulichen ebenso, dass zwischen den Betriebsgrößenklassen erhebliche Unterschiede in den Vollkosten der Milcherzeugung festzustellen sind. Aber auch innerhalb der verschiedenen Größenklassen variieren die Produktionskosten zum Teil beträchtlich. Das liegt vor allem daran, dass in einer Betriebsgrößenklasse Betriebe erfasst sind, die sich hinsichtlich der natürlichen Standortbedingungen, der Milchleistung, der Herdenrasse, der Altersstruktur der Maschinen und Gebäude sowie des Managements teilweise erheblich unterscheiden.

Neben den Durchschnittswerten ist daher die Darstellung der Variabilität der Ergebnisse für eine realistische Beurteilung der Situation von großer Bedeutung. 
Abbildung 5.4: $\quad$ Produktionskosten in Abhängigkeit von der Betriebsgröße

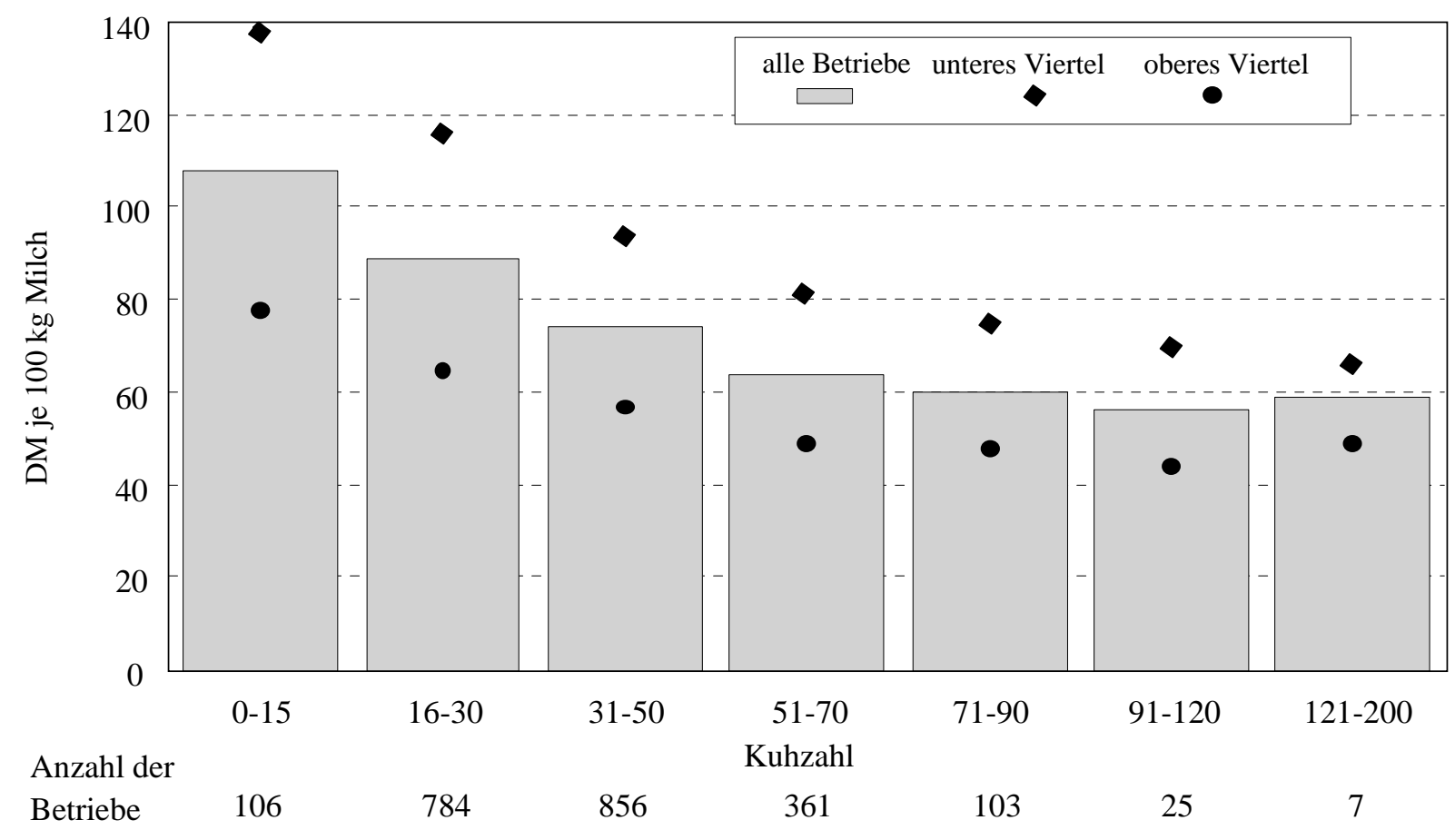

Quelle: Goertz (1999); Testbetriebsnetz des BML (1996/97).

\subsubsection{Produktionskostenanalyse mit IFCN-Daten}

Um Aussagen zur internationalen Wettbewerbsfähigkeit von Milchviehbetrieben abzuleiten, erfolgen im Rahmen des IFCN in regelmäßigen Abständen weltweite Produktionskostenvergleiche und detaillierte Ursachenanalyen für spezialisierte Milchviehbetriebe.

In Abbildung 5.5 sind beispielhaft die Vollkosten des Betriebszweigs Milch typischer IFCN-Betriebe im internationalen Vergleich dargestellt. Durch Gegenüberstellung mit dem Milchpreis lässt sich ermitteln, ob die Produktionskosten gedeckt sind. ${ }^{12}$

Der Produktionskostenvergleich in Abbildung 5.5 verdeutlicht, dass die Produktionskosten und die Milchpreise bei den untersuchten Betrieben deutlich variieren. Bei einem großen Teil der untersuchten Betriebe können die Vollkosten vollständig gedeckt werden. Es lassen sich vier Kostenbereiche erkennen: a) bis zu 15 US-\$: Argentinien, größere Betriebe in Indien/Pakistan, Westaustralien, Neuseeland, kleinere Betriebe in Victoria/Australien, 400-

12 Es handelt sich um Auszüge aus dem IFCN Dairy Report 2003 (HEMME et al., 2003). Auf die zugrunde liegende Methode der Produktionskostenberechnung wird in Kapitel 6.3.1 eingegangen. 
Kuhbetrieb in Estland; b) 15 bis 22 US-\$: Tschechische Republik, Brasilien, Bangladesh, größere Betriebe in Polen und bestimmte Betriebe in Indien und Pakistan, 35-Kuhbetrieb in Estland; c) 22 bis 30 US-\$: Belgien, Spanien, Dänemark, Ungarn, China und die größeren Betriebe in Deutschland, den Niederlanden, Frankreich, Irland und die USA als auch kleine Betriebe in Polen; d) > 30 US-\$: Fast alle kleinen Betriebe in Westeuropa, Israel, Kanada und den USA. ${ }^{13}$

Die IFCN-Betriebe können im Unterschied zum FADN nicht als repräsentativ für die nationale Milchwirtschaft angesehen werden, die Datenerhebung kann jedoch viel genauer dem eigentlichen Erhebungszweck zugeschnitten werden. Dies ermöglicht aussagekräftigere und detailliertere Kostenanalysen, wobei die Analyse der Ursachen für Kostenunterschiede zusammen mit den Unternehmern erfolgen kann.

Abbildung 5.5: $\quad$ Produktionskostenvergleich typischer IFCN-Betriebe für den Betriebszweig Milch (Teil 1)

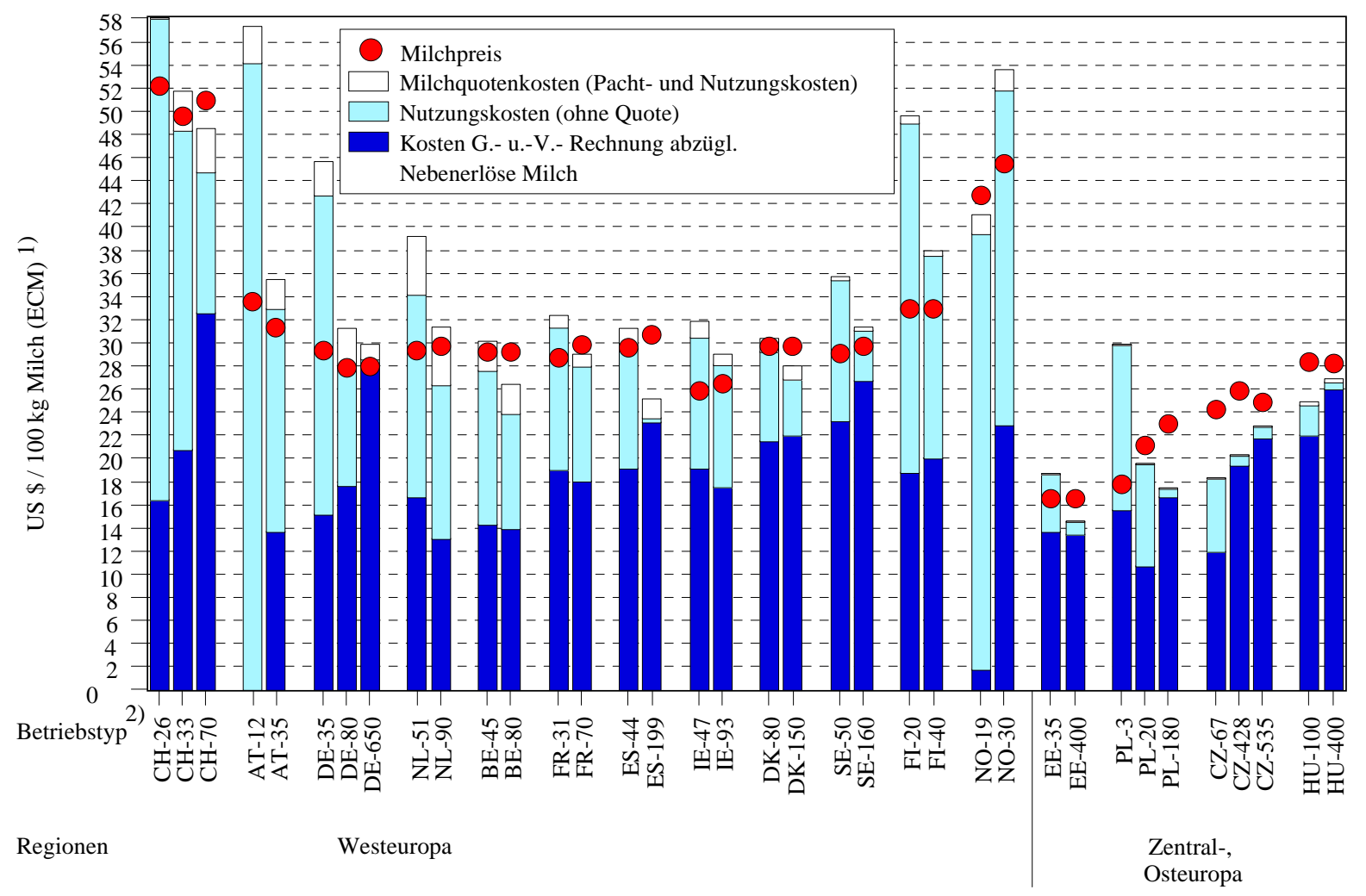

1) "Energie korrigierte Milch" $(E C M)=($ Milchproduktion * $((0,383 * \%$ Fett + 0,242 * \% Eiweiß + 0,7832) / 3,1138).

2) Buchstabe = Länderkürzel; Zahl = Anzahl Milchkühe.

Quelle: Hemme et. al. (2003).

13

Sowohl die Hintergründe für die Kostenstruktur in den einzelnen typischen Betrieben, als auch die Analyse der einzelnen Kostenpositionen im internationalen Vergleich sowie sonstige spezielle Studien zu den typischen IFCN-Betrieben sind im Dairy Report 2003 (HEMME et al., 2003, S. 24) nachzulesen. 
Abbildung 5.5: $\quad$ Produktionskostenvergleich typischer IFCN-Betriebe für den Betriebszweig Milch (Teil 2)

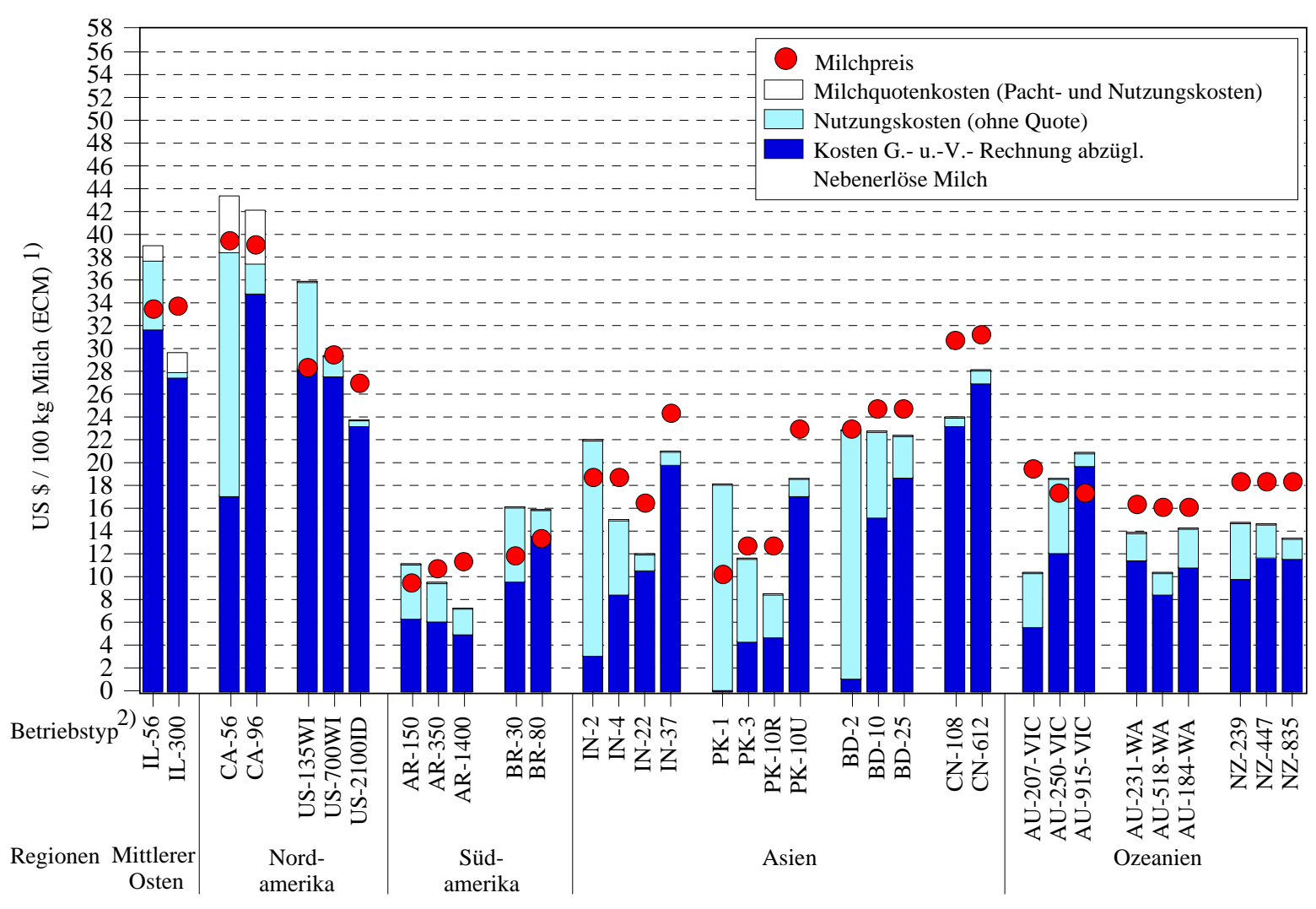

1) "Energie korrigierte Milch" $(E C M)=($ Milchproduktion * $((0,383 * \%$ Fett $+0,242 * \%$ Eiweiß $+0,7832) / 3,1138)$.

2) Buchstabe = Länderkürzel; Zahl = Anzahl Milchkühe.

Quelle: Hemme et. al. (2003).

\subsection{Einsatz von FADN- und IFCN-Daten für Politikfolgenabschätzun- gen auf dem EU-Milchmarkt}

Um aufzuzeigen, wie FADN und IFCN für Analysen zur Liberalisierung des EUMilchmarktes eingesetzt worden sind, werden im Folgenden zwei Studien vorgestellt, die verschiedene Politikoptionen untersuchen: eine Studie aus dem Jahr 2003 (HEMME et al., 2003) und eine Studie aus dem Jahr 2006 (ISERMEYER et al., 2006).

\subsubsection{Ergebnisse bei Anwendung der FADN-Methode}

Die Studie aus dem Jahr 2006 (IsERMEYER et al., 2006) ist vor dem Hintergrund der für 2008 vorgesehenen Zwischenbewertung der Agenda 2000 entstanden. In dieser Studie wird als Status-quo-Referenz (REF_SQ) die Umsetzung der Luxemburger Beschlüsse von 2003 einschließlich der später beschlossenen Zuckermarktreform zugrunde gelegt. Bei 
Milch beinhaltet dies die Verteilung der allgemeinen Zusatzquoten in den jeweiligen Mitgliedsstaaten sowie die Kürzung der Interventionspreise für Butter und Magermilchpulver. Die Direktzahlungen sind entsprechend der nationalen Umsetzung entkoppelt und in betrieblich bzw. regional differenzierte flächenbezogene Zahlungsansprüche für LF (ohne Dauerkulturen) überführt. Zusätzlich werden sich abzeichnende Politikänderungen berücksichtigt, und zwar:

- Anhebung der Modulation auf $13 \%$ für die $5.000 € /$ Betrieb übersteigenden Direktzahlungen.

- Kürzungen der 2. Säule-Zahlungen (Ausgleichszulage und Agrar-Umweltprämien) aufgrund der Neuabgrenzung der Gebietskulisse und Finanzplanung um 11,5 \%; differenziert nach Bundesländern.

- Bundesweite Übertragbarkeit der Milchquote.

Um die Auswirkungen von Politikänderungen auf Betriebsebene abschätzen zu können, wurde das komparativ-statische Programmierungsmodell FARMIS ${ }^{14}$ angewendet. Datengrundlage von FARMIS bilden die deutschen Testbetriebsdaten, die zu Betriebsgruppen aggregiert werden. In Abbildung 5.6 ist die Änderung der Milchproduktion differenziert nach Betriebsgröße (Zahl der Milchkühe im Basisjahr) und Region dargestellt.

Abbildung 5.6: Änderung der Milchproduktion, Referenz zu Basisjahr

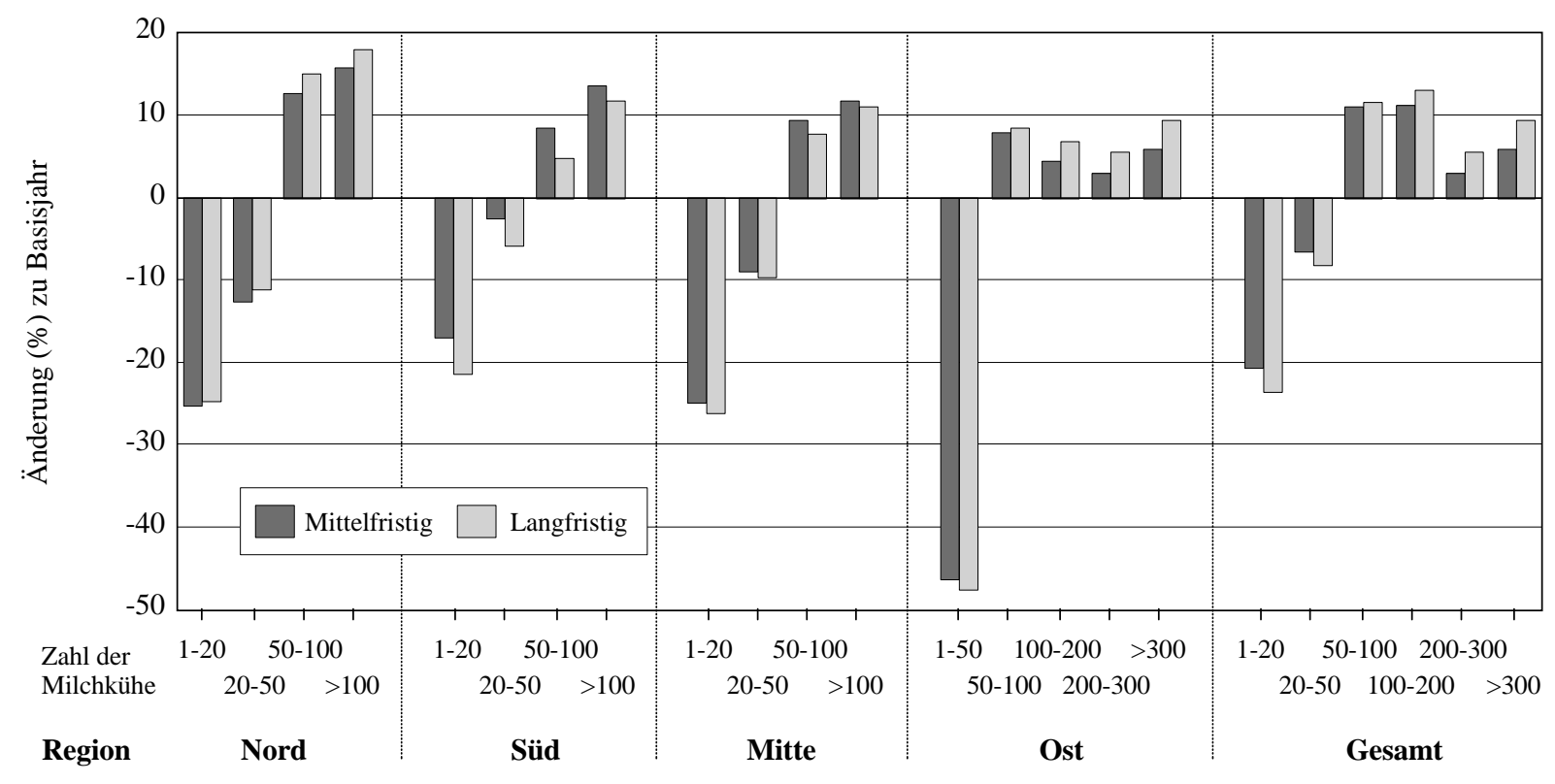

Quelle: Isermeyer et al. (2006).

14

Für eine Dokumentation des Modellsystems EU-FARMIS siehe BerTelsmeIER (2005), OfFERMANN et. al (2005) und KÜPKER et. al. (2006). 
Die Ergebnisse in Abbildung 5.6 zeigen, dass unter Fortführung der derzeitigen Politik (Referenzsituation) die Milchproduktion in vielen Betrieben unter 30 Kühen bis 2015 eingestellt wird. Die Wanderung der Milchproduktion wird im Wesentlichen durch die freiwerdende Milchquote bestimmt. Dies ermöglicht ein Wachstum der verbleibenden Betriebe. In allen Regionen steigt die Milchproduktion in den größeren Betrieben an, dies betrifft insbesondere die größeren Betriebe in den nördlichen Bundesländern.

Im Szenario „Übergang zu nationalem Quotenhandel“ deuten die FARMIS-Ergebnisse auf eine deutliche Annäherung der Quotenpreise in den alten und den neuen Bundesländern in der Referenz hin. Produktionsverlagerungen durch die Zusammenlegung der beiden Übertragungsgebiete halten sich daher in den Modellrechnungen in Grenzen (siehe Tabelle 5.1). Während kurzfristig eine Quotenübertragung Richtung Westen festzustellen ist, dreht sich dieser Trend in der langfristigen Betrachtung aufgrund der günstigeren Größenstruktur der Milchviehbetriebe in den östlichen Bundesländern um.

Tabelle 5.1: $\quad$ Änderung der Milchproduktion in Prozent bei Übergang von zwei Übertragungsregionen zu nationalem Quotenhandel

\begin{tabular}{lcc}
\hline & Kurzfristig & Langfristig \\
\hline Bayern & 0,1 & $-1,1$ \\
Baden-Württemberg & 0,2 & $-1,1$ \\
Hessen & 0,2 & $-1,3$ \\
Nordrhein-Westfalen & 0,2 & $-1,3$ \\
Niedersachsen & 0,2 & $-1,3$ \\
Rheinland-Pfalz & 0,1 & $-1,0$ \\
Schleswig-Holstein & 0,2 & $-1,3$ \\
Saarland & 0,2 & $-1,1$ \\
Brandenburg & $-0,6$ & 4,6 \\
Mecklenburg-Vorpommern & $-0,6$ & 4,7 \\
Sachsen & $-0,6$ & 4,4 \\
Sachsen-Anhalt & $-0,6$ & 4,2 \\
Thüringen & $-0,6$ & 4,3 \\
\hline
\end{tabular}

Quelle: Isermeyer et al. (2006).

Eine 50 \%ige Kürzung der Ausgleichszahlungen für benachteiligte Gebiete und der Agrarumweltprämien führt nur zu geringen Produktionswirkungen (siehe Abbildung 5.7). In den kleinen Milchviehbetrieben in den südlichen und östlichen Bundesländern fallen sie mit 2,0 bzw. -1,0 \% am höchsten aus. Ferner konnte gezeigt werden, dass durch die Reduzierung der 2. Säule-Prämien der Anreiz, landwirtschaftliche Flächen nicht zu nutzen bzw. nur minimal zu pflegen (Mulchen), deutlich ansteigt. 
Abbildung 5.7: Änderung der Milchproduktion bei 50 \%iger Kürzung der LFA+AUP, \% zu REF_SQ

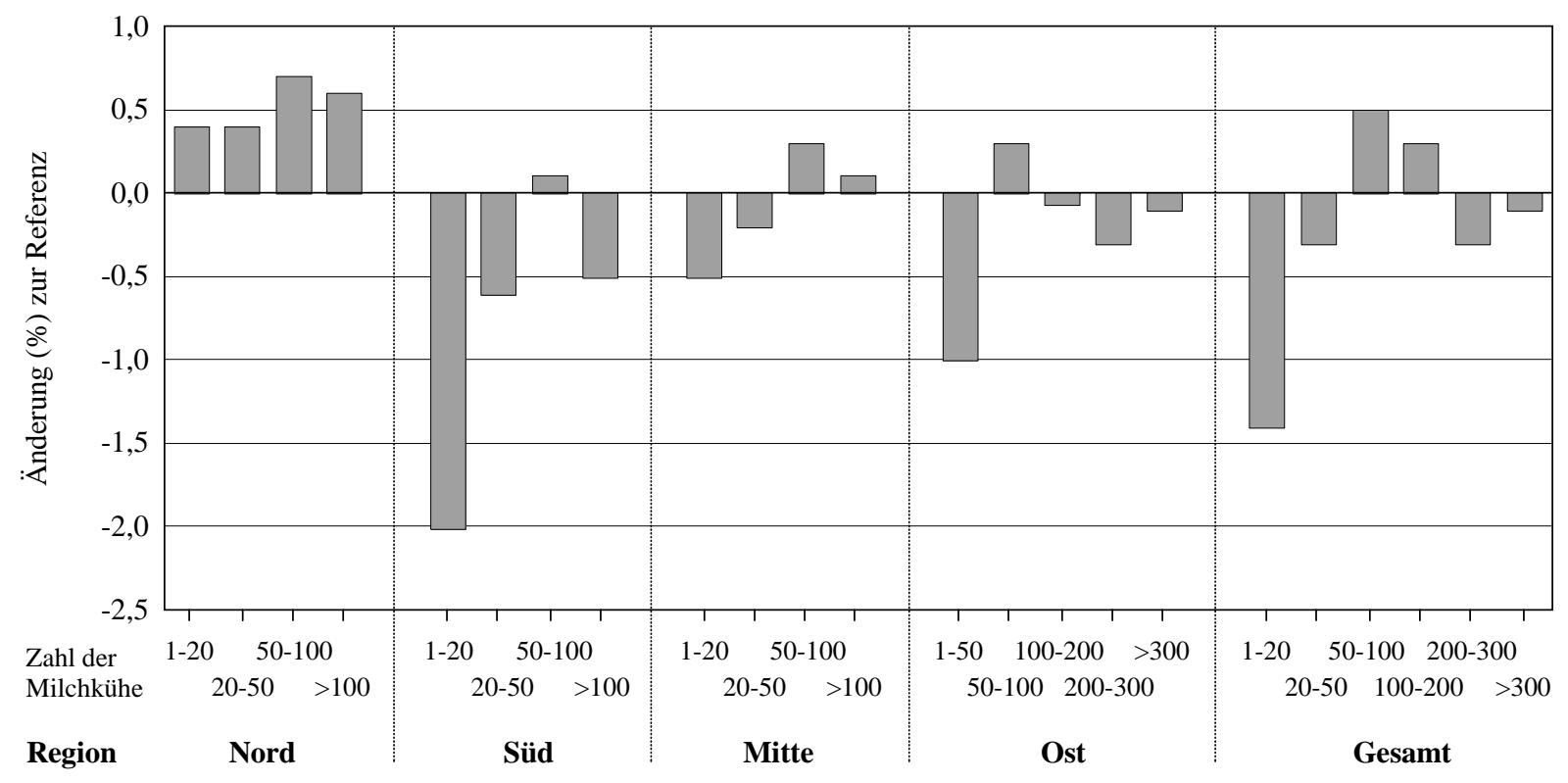

Quelle: Isermeyer et al. (2006).

Die Einkommenswirkungen (NWSF/AK ${ }^{15}$ ) fallen insbesondere in den kleineren Betrieben deutlicher aus als die Produktionswirkungen und liegen beispielsweise in den Betrieben bis 20 Kühen in den südlichen Bundesländern im Schnitt bei -10,8 \%. Nach den Modellergebnissen sind zwar keine unmittelbaren Auswirkungen auf die Milchproduktion zu erwarten, dennoch lassen die prognostizierten Einkommensrückgänge in kleineren Betrieben einen leicht beschleunigten Strukturwandel erwarten.

Bei Beibehaltung der Quotenregelung und Kürzung der Quoten um 15 \% gegenüber der Status-quo-Referenz wird die Quote in Deutschland ausgeschöpft. Die Verknappung der Quote und der Anstieg der Milchpreise führen zu einem deutlichen Anstieg der Preise für Milchquote, die zu einem jährlichen Aufwand von 7,5 bis 9,4 ct/kg für Fremdquote führen. Im Vergleich zur Status-quo-Referenz liegen die Einkommen in fast allen Betriebsgruppen auch im Quotenkürzungsszenario niedriger (ISERMEYER et al., 2006, Tabellen A.4.2 und A.4.3). Der gegenüber der Status-quo-Referenz nur geringfügig höhere Erzeugerpreis reicht nicht aus, um die Reduzierung der Einkommensbasis in Folge der Quotenkürzung zu kompensieren. Die Reduzierung der Milchproduktion durch die Quotenkürzung lässt die Nachfrage nach Futterflächen weiter sinken und führt zu etwas geringeren Pachtpreisen für Boden. 
Eine belastbare EU-weite Abschätzung der grenzüberschreitenden Produktionsverlagerungen, die sich bei einem Ausstieg aus der Quote ergeben würden, ist mit den im Rahmen der vorgestellten Studie verfügbaren Modellen noch nicht möglich.

Der Strukturwandel ist bei diesem Ansatz exogen vorgegeben, die Entscheidung zur Betriebsaufgabe wird nicht explizit modelliert. Es handelt sich um einen komparativstatischen Modellansatz, was bedeutet, dass es keinen Zeitpfad gibt. Das Investitionsverhalten kann daher nur vereinfacht abgebildet werden.

Im Gegensatz zur erfolgten komparativ-statischen Betrachtung mit Testbetriebsdaten werden im Folgenden Politikfolgenabschätzungen mit IFCN-Daten im Rahmen eines dynamischen Modellansatzes vorgestellt.

\subsubsection{Ergebnisse bei Anwendung der IFCN-Methode}

Die Studie aus dem Jahr 2003 (HEMmE et al., 2003) befasst sich mit der Zwischenbewertung der Agenda 2000 (Mid-term Review). Es standen zu dem Zeitpunkt vor allem folgende zwei Politikreformen im Mittelpunkt der Diskussion: die Agenda-Zwischenbewer-tung (Mid-term Review) und darüber hinaus die Abschaffung der Milchquotenregelung im Jahr 2008.

Folgende Szenarien wurden analysiert:

- Referenzszenario Agenda 2000 ${ }^{16}$ („AG Ref.“): Mit Milchquotenregelung, Milchpreis in 2008 -11,2 \% zu 1999, Milchprämien 2,5 €/100 kg Milch, Quotenaufstockung.

- MTR (Beschluss Juni 2003, „MTR MM“): Mit Milchquotenregelung, Milchpreis in 2008 -15 \% zu 1999, Milchprämien 3,55 €/100 kg Milch; mit Entkopplung/Modulation $^{17}$; Quotenaufstockung.

- Milchquotenausstieg („ohne Quote“): Quotenausstieg 2008 bei gleicher Entwicklung bis 2007 und Prämien wie im Szenario „MTR MM“, Milchpreis in 2008 -22 \% zu 1999.

Ziel ist es, die Auswirkungen verschiedener Politiken auf die Milchviehbetriebe zu untersuchen. Dazu wird im Folgenden mit dem IFCN-Ansatz ein Vergleich der Gewinnentwicklung in der „Baseline“ (Referenzsituation, s. o.) und bei den berücksichtigten Politikszenarien (s. o.) vorgestellt, der Aussagen über die Auswirkungen verschiedener Politiken auf

16 Siehe Kapitel 4.5.2.

17 Da die Entkopplung und Modulation bis zum Abschluss dieser Berechnungen noch nicht spezifiziert waren, sind sie hier nicht abgezogen worden. 
die Milchviehbetriebe ermöglicht. Die Ergebnisse werden im Hinblick auf die Fragestellung dieser Arbeit konzentriert. Detaillierte Informationen sind im Dairy Report 2003 (HEMME et al., 2003, S. 106-113) nachzulesen.

\section{Entwicklung des Gewinns und der Direktzahlungen in der Baseline (Agenda 2000)}

Zunächst werden die Entwicklungen des Gewinns und der Direktzahlungen in der Referenzsituation im Vergleich dreier EU-Länder anhand von sechs Betrieben exemplarisch dargestellt. ${ }^{18}$

Wie Abbildung 5.8 zeigt, erhöhten die deutschen Betriebe von 1999 bis 2001 ihre Einkommen, bedingt durch ansteigende Milchpreise. Sinkende Milchpreise in 2002 führten dazu, dass sich das Einkommen im Jahr 2002 wieder reduzierte und dann für den restlichen Untersuchungszeitraum nominal konstant blieb. ${ }^{19}$

Die Einkommen der schwedischen Betriebe stiegen bis zum Jahr 2001/2002. In diesem Zeitraum erfolgte die Zahlung einer Grünlandprämie von 120 bis $130 €$. Anders als in Deutschland blieben die Milchpreise in diesem Zeitraum konstant. Das Einkommen des schwedischen 160-Kuhbetriebes ist stärkeren Schwankungen ausgesetzt als das des schwedischen 50-Kuhbetriebes. Die Gewinnmarge des schwedischen 160-Kuhbetriebes ist geringer als die Gewinnspanne des schwedischen 50-Kuhbetriebes. Änderungen in den Milchpreisen oder Direktzahlungen haben demzufolge höhere Einkommensänderungen zur Folge.

Bei den spanischen Betrieben ist zu erwähnen, dass die Milchpreisentwicklung im Zeitraum 1999 bis 2003 für beide Betriebe unterschiedlich ist. Der spanische 42-Kuhbetrieb zeigt von 1999 bis 2004 ähnliche Einkommensentwicklungen wie die deutschen Betriebe. Mit der Einleitung der Agenda 2000-Maßnahmen fallen die Einkommen leicht. Der spanische 175-Kuhbetrieb hat höhere Einkommenseinbußen in 2002, weil die Milchpreise stärker fallen als im 42-Kuhbetrieb. In den Folgejahren führt eine im Vergleich mit dem 42Kuhbetrieb geringere Preissenkung zu nominal konstanten Einkommen.

18

Den hier zusammengefassten Ergebnissen liegen die in Kapitel 6.3.1.2 beschriebene Methode zur Produktionskostenberechnung und die in Kapitel 6.4 beschriebenen Annahmen zur Projektion der IFCNBetriebe zugrunde. 
Abbildung 5.8: $\quad$ Projektion der Entwicklung von typischen Milchviehbetrieben (IFCN) in Deutschland, Schweden und Spanien in der Baseline (1999 bis 2008)
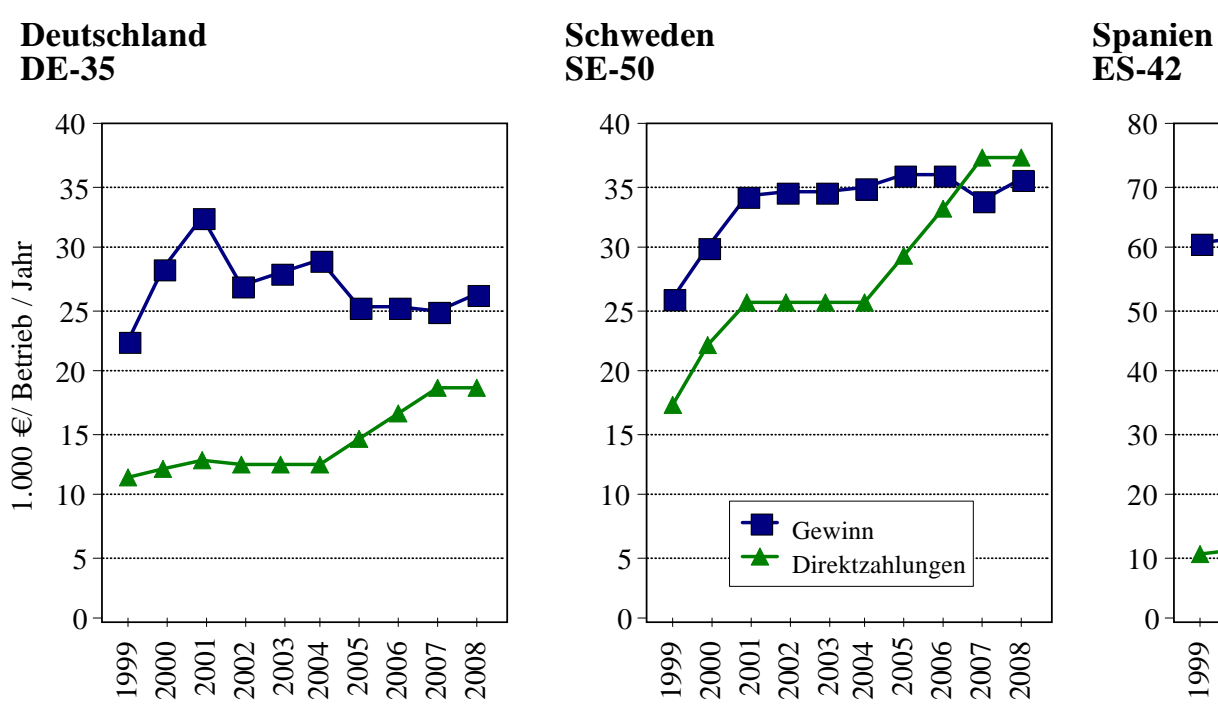

DE-68

\section{SE-160}

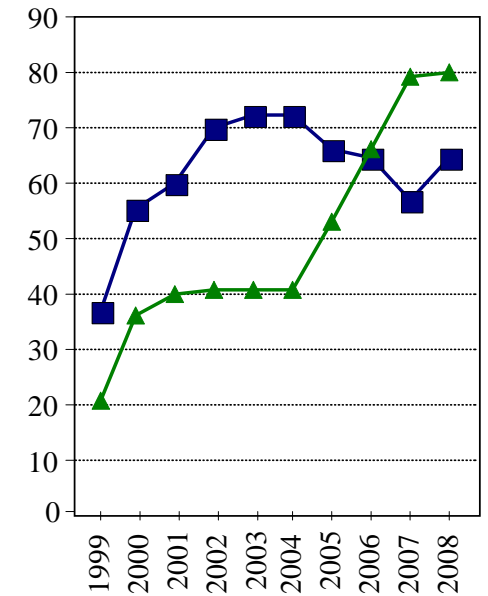

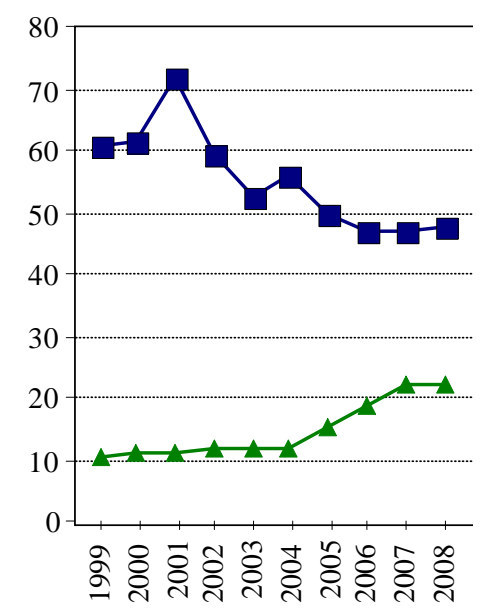

ES-175

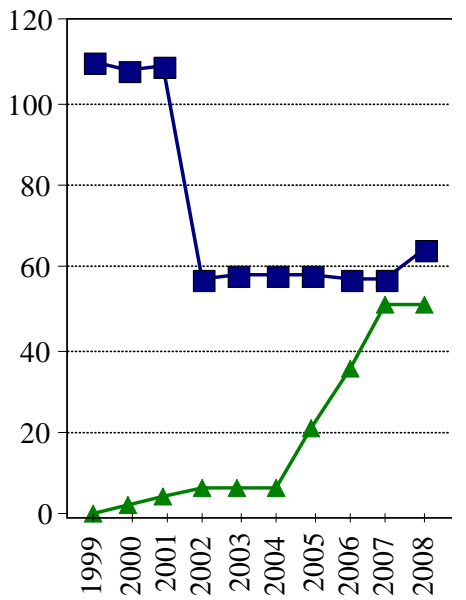

Quelle: Dairy Report 2003, Kapitel 4.10, Seite 111.

\section{Auswirkungen des MTR-Beschlusses und eines fiktiven Milchquotenausstiegs auf die Einkommen}

Aufgrund der besseren Informationsgrundlage erfolgt die Politikfolgenabschätzung zum MTR und Quotenausstieg nur am Beispiel der drei deutschen Betriebe. Die Einkommenseffekte verglichen mit der Referenzsituation (Agenda 2000) sind in Abbildung 5.9 dargestellt.

Im Vergleich zur Agenda 2000 führt die vorgezogene Reform des Milchmarktes in 2004 aufgrund der vorgezogenen Milchpreissenkung (-3€/100 kg Milch) zu Einkommenseinbußen (-10 bis -20\%), die auch durch steigende Direktzahlungen $(1,83 € / 100 \mathrm{~kg})$ nicht kompensiert werden. Erst in 2006 wird die volle Höhe der Direktzahlungen gewährt. 
Abbildung 5.9: $\quad$ Folgenabschätzung des MTR-Beschlusses und eines fiktiven Milchquotenausstiegs am Beispiel von drei deutschen IFCN-Betrieben
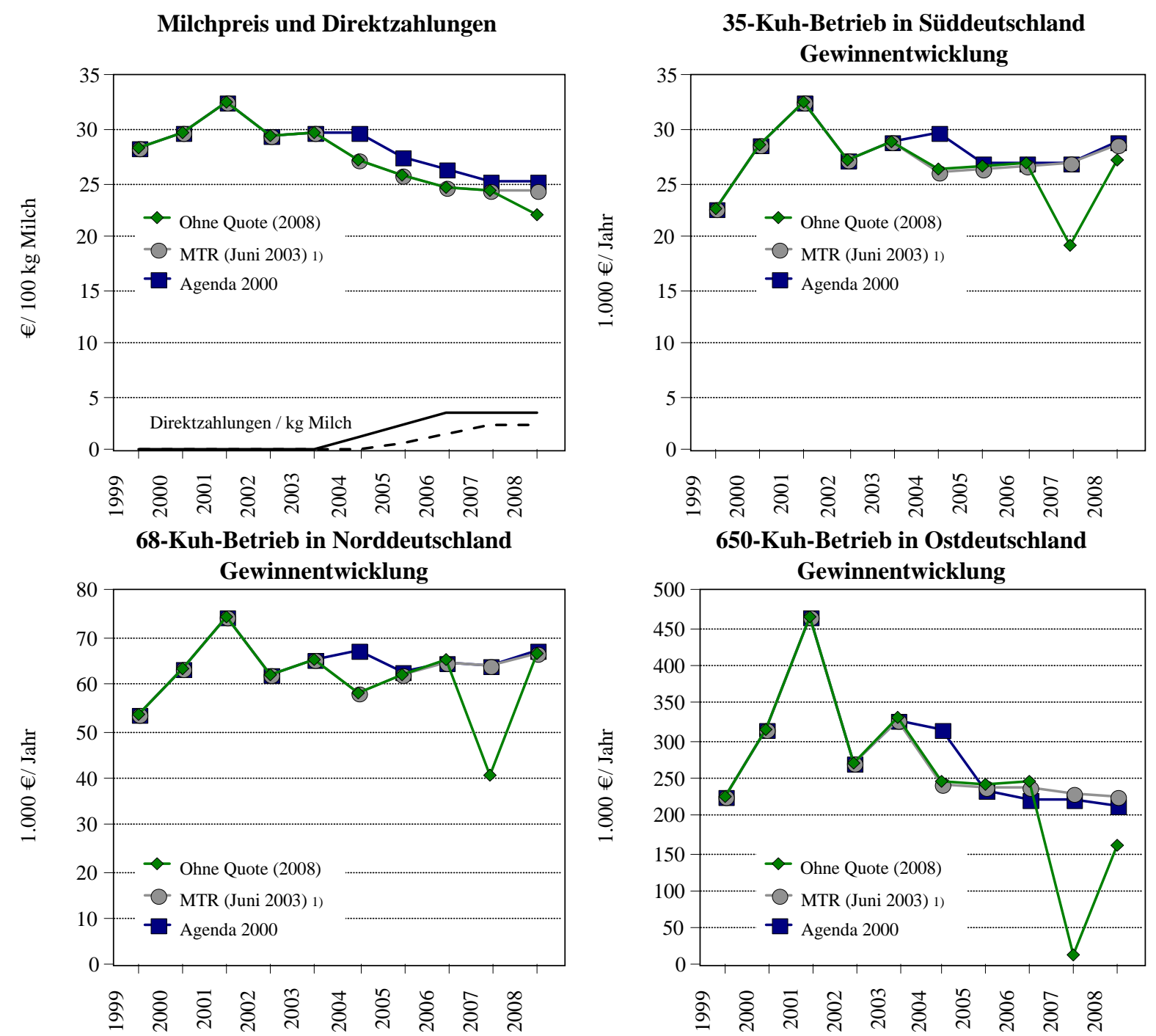

Einkommenseffekte in 2008 verglichen mit der Baseline

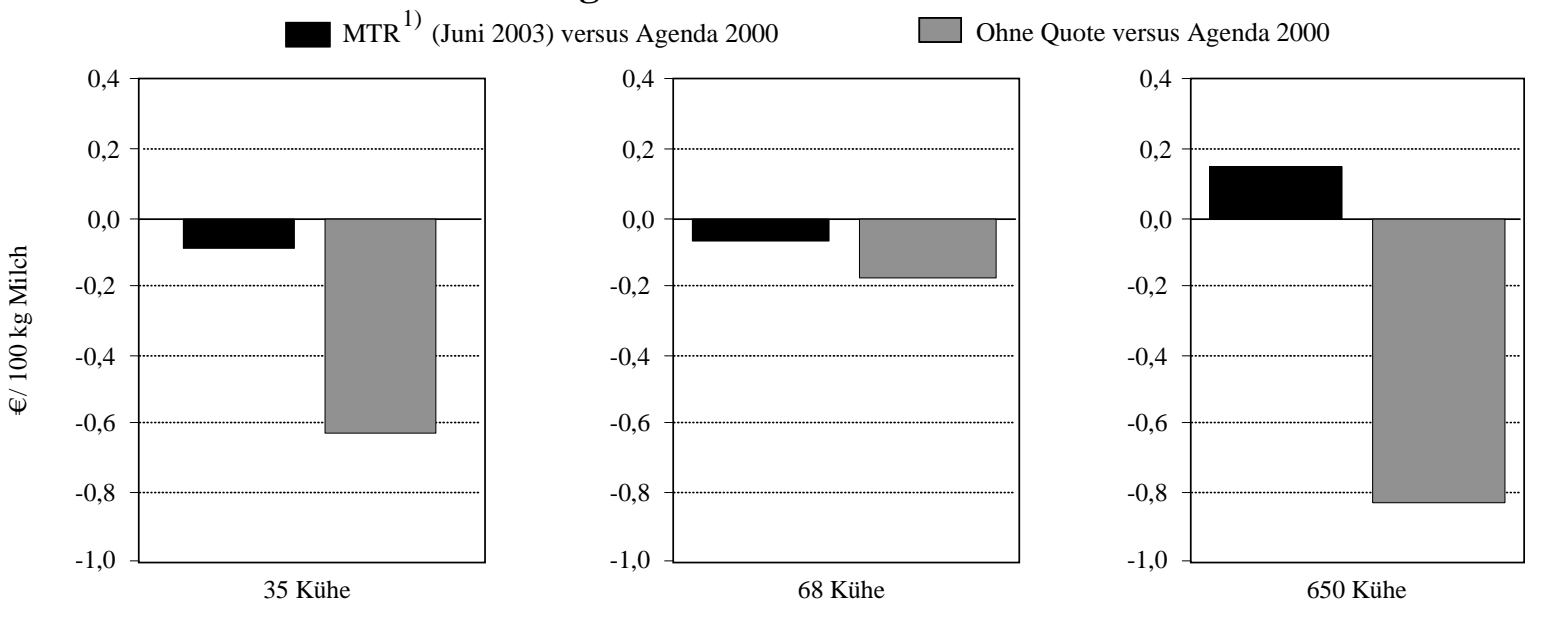

1) MTR = Zwischenbewertung der Agenda 2000.

Quelle: Eigene Berechnung und Darstellung, Hemme et al., Dairy Report 2003, Kapitel 4.9, Seite 109. 
Der MTR Beschluss bewirkt im Vergleich zur Agenda 2000 für die Untersuchungsbetriebe keine signifikanten Einkommensverluste $( \pm 0,2 € / 100 \mathrm{~kg}$ Milch). Die Milchpreissenkung von $1 € / 100 \mathrm{~kg}$ Milch wird durch zusätzliche Direktzahlungen kompensiert. Der ostdeutsche Betrieb profitiert vom Mehrwertsteuersystem, während die westdeutschen Betriebe (D-35, D-68) keine Mehrwertsteuereinnahmen und -ausgaben saldieren. Durch die Erzeugerpreissenkung werden auch die Mehrwertsteuererlöse sinken.

Im Szenario Quotenausstieg schwanken die Einkommenseffekte zwischen -0,15 und -0,8 €/100 kg Milch. Stärkere Preissenkungen können durch sinkende Quotenrenten und Abschreibungen kompensiert werden. Ein hoher Anteil an Pachtquote beim 68-Kuhbetrieb in Norddeutschland führt im Vergleich zu Betrieben mit Milchquotenzukauf zu geringeren Einkommenseinbußen nach dem Quotenausstieg. Der starke Rückgang der Einkommen in 2007 ist bedingt durch die einmalige Abschreibung der gekauften Milchquote.

\section{Einkommenseffekte bei Berücksichtigung betrieblicher Anpassungsreaktionen}

Im Folgenden wird erläutert, wie die politikbedingten Einkommenseffekte bei Berücksichtigung betrieblicher Anpassungsreaktionen ausfallen. Es werden zwei Anpassungsstrategien analysiert:

1. Die Ausnutzung der vorhandenen Stallkapazitäten, d. h. die Ausdehnung der Milchproduktion im Rahmen der zur Verfügung stehenden Stallkapazitäten ohne Investitionen in Stallungen und Melkanlage.

2. Der Stallanbau, d. h. die zusätzliche Ausdehnung der Produktion über einen Stallanbau.

Diese Strategien sind zusammen mit Wissenschaftlern und den zuständigen Beratern und Landwirten vor Ort entwickelt worden. Nähere Details zu den analysierten Wachstumsschritten sind bei DEEKEN und HEMME (2002) nachzulesen. ${ }^{20}$

Die Abschätzung von betrieblichen Anpassungsreaktionen zeigt für die oben analysierten deutschen IFCN-Betriebe folgende Ergebnisse (vgl. DEEKEN und Hemme, 2002):

- Durch Wachstum sind die Betriebe in der Lage, ihre Gewinnschwelle bei Ausnutzung der vorhandenen Stallkapazitäten ${ }^{21}$ um 1 bis $4 € / 100 \mathrm{~kg}$ Milch und bei einem Anbau

\footnotetext{
20 Vgl. auch Kapitel 7.2.
}

21 35-Kuhbetrieb: Wachstum ohne Investition auf 43 Kühe; 68-Kuhbetrieb: Wachstum ohne Investition auf 80 Kühe; 650-Kuhbetrieb: Wachstum ohne Investition auf 800 Kühe; für detailliertere Informationen siehe DEEKEN und HEMmE (2002). 
um 1 bis $11 € / 100 \mathrm{~kg}$ Milch zu reduzieren. Die durchschnittlichen Grenzkosten ${ }^{22}$, die den Betrieben bei einem Wachstum entstehen, werden bei der Ausnutzung der vorhandenen Stallkapazitäten auf 16 bis $23 € / 100$ kg Milch und bei einem Anbau auf 17 bis $21 €$ geschätzt.

- Die Strategie „Stallanbau“ (Wachstum auf 70 Kühe $^{23}$ ) scheidet für den 35-Kuhbetrieb aufgrund der hohen Gewinnschwelle aus. Die Ausnutzung seiner Stallkapazitäten zu geringen Grenzkosten (Ausweitung der Produktion um ca. 23 \%) ermöglicht ihm hingegen, seine Produktion mittelfristig rentabler fortzuführen als ohne Anpassung. Ein Stallanbau (Wachstum auf 120 Kühe $^{24}$ ) ermöglicht dem 68-Kuhbetrieb, seine Gewinnschwelle auf $23 € / 100 \mathrm{~kg}$ Milch zu reduzieren. Durch diesen Wachstumsschritt würde der 68-Kuhbetrieb bei Fortführung der Milchquotenregelung seine Vollkosten decken.

- Beide Wachstumsschritte, Wachstum ohne Investition auf 800 Kühe und Wachstum mit Investition auf 1.000 Kühe, führen beim 650-Kuhbetrieb zur Deckung seiner Vollkosten. Da die geschätzten Grenzkosten (22 und $21 €$ ) relativ nah am Milchpreis liegen, sind Investitionen jedoch risikobehaftet (vgl. DEEKEN und HEMME, 2002).

- Grundsätzlich lässt sich feststellen, dass sich die wirtschaftliche Situation durch verstärktes Wachstum (Einkommenssteigerung bis 2,4 €/100 kg Milch nach einem Quotenausstieg) in allen Betrieben verbessert.

Wie dieses Beispiel zeigt, würde die Politikfolgenabschätzung ohne Berücksichtigung realitätsnaher Anpassungsreaktionen zu falschen Einschätzungen gelangen.

22 Bei DeEKen und Hemme (2002) wurden die zusätzlichen Kosten der Milchproduktion wie folgt berechnet: variable Kosten der Produktion, zusätzliche Abschreibungen und Zinsen für Investitionen, zusätzliche Arbeit, zusätzliche Fixkosten) - Nebenerlöse im Betriebszweig Milch (Rindfleischerlöse, Kälber- und Färsenverkäufe, Direktzahlungen im Betriebszweig Milch); Berechnungen sind ohne Quotenkosten und ohne Milchprämien für die zusätzliche Milch durch Wachstum. 


\subsection{Schlussfolgerungen}

Die Ergebnisdarstellung mit FADN- und IFCN-Daten offenbart die potenziellen Stärken und Schwächen beider Ansätze, wie bereits in der Problemstellung (Kapitel 1.1) angedeutet:

- Mit Hilfe der IFCN-Methode ist es möglich, differenziert nach Betriebstypen u. a. festzustellen,

- $\quad$ welche Einkommenseinbußen bei Änderung der politischen Rahmenbedingungen ohne/mit Anpassung mittel-/langfristig zu erwarten sind,

- $\quad$ welche Ursachen den Einkommensentwicklungen zugrunde liegen,

- welche Anpassungsstrategie betriebswirtschaftlich am geeignetesten bzw. realistischsten ist, um auch mittel-/langfristig rentabel zu produzieren,

- welche Kosteneinsparungen durch betriebliche Anpassungen erzielt werden können und

- welche Ausweitung der Produktion bei betrieblicher Anpassung zu erwarten ist.

- Mit Hilfe der FADN-Daten (bzw. nationalen Testbetriebsdaten) ist es möglich,

- $\quad$ kurzfristig Einkommenseinbußen bei Änderung der politischen Rahmenbedingungen festzustellen,

- Tendenzen zur Ausweitung, Stagnation oder Einschränkung der Milchproduktion für die EU-Regionen zu ermitteln, ${ }^{25}$

- Aussagen über die regionale Wanderung der Milchproduktion zu treffen,

- Effekte auf andere Märkte abzuschätzen (z. B. Auswirkungen einer Reduzierung der Milchproduktion (über die Nachfrage nach Futterflächen) auf die Pachtpreise für Boden),

- die Varianz in den Ergebnissen zu messen, Verteilungen zu analysieren und damit auch Aussagen zur individuellen Betroffenheit zu geben.

25 Auch mit einer anderen Methode, zum Beispiel mit einem linearen Programmierungsansatz (siehe Kapitel 3.2.3: KLEINHANSS et al., 2001), sind Anpassungsreaktionen bzw. Wachstumsstrategien nur in ganz begrenztem Umfang zu ermitteln (kaum Mengenänderung). 
Es wird deutlich, dass für kurzfristige Abschätzungen ohne nennenswerte Mengenänderungen die FADN-Daten gute Voraussetzungen bieten. Die potenziellen Stärken der IFCN-Methode liegen aufgrund seines Praktikeransatzes ${ }^{26}$ (siehe Kapitel 4.3) in der Ursachenanalyse und in der Ermittlung realitätsnaher, betrieblicher Anpassungsreaktionen. Mit den repräsentativen FADN-Daten sind aufgrund der Erhebungsmethodik (siehe Kapitel 4.2) allenfalls geringe Mengenänderungen kurzfristig abzuschätzen. Zur Abbildung des betrieblichen Anpassungsverhaltens mit FADN sind zusätzliche Informationen notwendig. Aus den differenzierten und realitätsnahen Informationen zum Anpassungsverhalten der IFCN-Betriebe sind andererseits keine Rückschlüsse auf Regions- bzw. Mitgliedsländerebene ableitbar.

Der Überblick über die in der quantitativen Analyse zur EU-Milchmarktpolitik verwendeten Datengrundlagen verdeutlicht, dass

- der Aufwand zur Harmonisierung und Ergänzung der bisher nur regional oder national verfügbaren Datenbestände sehr hoch ist und innerhalb dieser Studie nicht zu leisten ist.

- der Vorteil der FADN- bzw. nationalen Testbetriebsdaten in der Repräsentativität und EU- bzw. deutschlandweit harmonisierten Erhebung der Daten liegt. Der FADNDatenbasis fehlt aber die für eine detaillierte Kostenanalyse und für eine zukunftsgerichtete Modellierung erforderliche Datentiefe (z. B. getrennte Ausweisung von Mengen und Preisen für Inputs). Es besteht kein Zugriff auf einzelbetriebliche Daten und keine Rückkopplung zu den Betrieben, die im FADN verzeichnet sind. Ferner stehen die FADN-Daten mit einer zeitlichen Verzögerung von ca. zwei Jahren zur Verfügung.

- die Anwendung des IFCN-Ansatzes den Vorteil hat, dass die betrieblichen Anpassungsreaktionen erfasst und quantifiziert werden. Zudem werden die Vollkosten der Betriebe aufgrund von detaillierten Preis- und Mengendaten erhoben. Die Erhebung der Betriebe erfolgt nach einem einheitlichen Erfassungskonzept, womit sie internationale Kompatibilität erhalten. Im Gegensatz zu Buchführungsstatistiken sind diese Daten zeitnah. Einzelbetriebliche Besonderheiten werden im Panelansatz eliminiert. Ein Nachteil ist, dass die Repräsentativität im statistischen Sinne aufgrund der geringen Anzahl von Betrieben pro Land nicht gegeben ist. 


\section{Verknüpfungsansatz I: Einordnung und Auswahl der IFCN-Betriebe}

Ziel der in dieser Arbeit zu entwickelnden Verknüpfungsansätze ist es, durch eine kombinierte Anwendung von FADN- und IFCN-Datensätzen bei Politikfolgenbewertungen verbesserte Ergebnisse zu erzielen. Konkret soll beim ersten Verknüpfungsansatz untersucht werden, wie mit Hilfe von FADN-Daten eine erhöhte Aussagekraft der Ergebnisse typischer IFCN-Betriebe erzielt und wie die Auswahl der IFCN-Betriebe auf eine breitere Basis gestellt werden kann.

In Kapitel 6.1 wird das Konzept zur Verknüpfung von FADN- und IFCN-Datensatz zunächst im Überblick dargestellt. Anschließend wird in den Kapiteln 6.2 und 6.3 auf die angewendeten methodischen Fragen und Daten im Detail eingegangen. Dazu zählen die Selektion von FADN-Vergleichsbetrieben mit Hilfe der euklidischen Distanz, die statistische Einordnung typischer IFCN-Betriebe und die Schätzung der FADN-Produktionskosten der Milcherzeugung. In Kapitel 6.4 wird die Leistungsfähigkeit des entwickelten Verknüpfungskonzeptes überprüft und bewertet. Zum Abschluss wird ein Verfahren vorgestellt, wie die Auswahl von typischen IFCN-Betrieben auf Basis von FADN-Daten erfolgen kann.

\subsection{Verknüpfungskonzept im Überblick}

Das internationale Netzwerk typischer IFCN-Betriebe weist in Relation zu FADN eine geringe Anzahl an Betrieben auf, die aber mit sehr großer Datentiefe erhoben werden. Im Rahmen des IFCN-Panelansatzes werden wichtige detaillierte Informationen insbesondere zu typischen, betrieblichen Anpassungsreaktionen generiert, die bei der Modellierung berücksichtigt werden. Mit Hilfe der im statistischen Sinne repräsentativen FADN-Daten wird versucht, die Aussagekraft der Ergebnisse typischer IFCN-Betriebe zu verbessern. Dabei stellen sich folgende Fragen:

- Für wie viele und für welche Betriebe aus der Grundgesamtheit sind die IFCNErgebnisse aussagekräftig?

- Welchen Anteil an der sektoralen Produktion deckt der typische Betrieb in der Region/im Land ab?

- Wie typisch sind die IFCN-Betriebe vor dem Hintergrund der Verteilung in der Grundgesamtheit? Können Anregungen gegeben werden für Korrekturen bzw. für die Neukonzeption bei der Auswahl von IFCN-Betrieben?

- Wie kann der FADN-Datensatz nutzbar gemacht werden für eine künftig verbesserte Betriebsauswahl in IFCN? 
Dazu muss jeder IFCN-Betrieb vor dem Hintergrund der FADN-Daten eingeordnet werden. Das Konzept zur statistischen Einordnung der typischen IFCN-Betriebe wird in Kapitel 6.1.1 beschrieben.

\subsubsection{Konzept zur Einordnung der IFCN-Betriebe}

Die Einordnung der IFCN-Betriebe erfolgt in einem zweistufigen Prozess. In der ersten Stufe werden die IFCN- und FADN-Daten zunächst anhand struktureller Kennzahlen verglichen. Darauf aufbauend werden die FADN-Betriebe ausgewählt, die den IFCNBetrieben strukturell am ähnlichsten sind.

In der zweiten Stufe werden die IFCN- und die ausgewählten FADN-Betriebe (Stufe 1) hinsichtlich ihrer wirtschaftlichen Leistungskennzahlen verglichen und eingeordnet, um zu erkennen, wie der IFCN-Betrieb hinsichtlich seines wirtschaftlichen Erfolges ${ }^{1}$ und damit die Simulationsergebnisse einzuschätzen sind.

Die Auswahl der Vergleichsbetriebe erfolgt in Anlehnung an das multivariate Analyseverfahren der Clusteranalyse ${ }^{2}$. Es werden dabei simultan verschiedene Vergleichsmerkmale für jeden ausgewählten typischen IFCN-Betrieb berücksichtigt. Die Ähnlichkeit zwischen zwei Betrieben wird anhand der euklidischen Distanz ${ }^{3}$ ermittelt (FENNEKER, 2002, S. 194).

Bei der Auswahl der Vergleichsbetriebe werden für einen typischen Untersuchungsbetrieb die strukturell ähnlichen FADN-Betriebe in der Reihenfolge ihrer „Nähe“ zum IFCNBetrieb gegenübergestellt. Das euklidische Distanzmaß reflektiert in diesem Fall die „Nähe“ bzw. die strukturelle Ähnlichkeit der FADN-Betriebe zum typischen Betrieb.

Um den typischen IFCN-Betrieb in der Region und im Land besser einordnen sowie Rückschlüsse ziehen zu können, für wie viele und welche Betriebe aus der Grundgesamtheit die IFCN-Ergebnisse aussagekräftig sind, werden die Anzahl der Betriebe in der Grundgesamtheit und die regionale Milchproduktion (absolut und relativ) mittels Hochrechnung ermittelt.

Genauere methodische Details zur Auswahl der FADN-Vergleichsgruppe finden sich in Kapitel 6.2/6.2.1.

\footnotetext{
1 Angenommene Indikatoren: Betrieblicher Gewinn und Produktionskosten.

2 Siehe Kapitel 6.2.1.

$3 \quad$ Siehe Kapitel 6.2.1.
} 
Für die Einordnung der typischen Betriebe in die kumulierte Verteilung der FADNBetriebe wird ein eigener Lösungsansatz entwickelt. Dieser Lösungsweg beinhaltet u. a. die Einordnung der typischen Betriebe anhand der Produktionskosten. Der Vergleich der wirtschaftlichen Leistungskennzahlen soll Rückschlüsse darüber erlauben, wie typisch die IFCN-Betriebe vor dem Hintergrund der Verteilung in der Grundgesamtheit wirklich sind. Die FADN-Daten müssen für die Produktionskostenschätzung zunächst aufbereitet werden, da im Betriebsbogen des FADN im Vergleich zu IFCN nicht vorgesehen ist, betriebszweigspezifische Kosten getrennt aufzuführen. Auf die Schätzung der betriebszweigspezifischen Produktionskosten bei den FADN-Betrieben wird in Kapitel 6.3.1 eingegangen.

Als Ergebnis dieses Selektions- und Einordnungsprozesses erhalten wir eine bestimmte Anzahl von FADN-Betrieben, die dem IFCN-Betrieb bezüglich Struktur hinreichend ähnlich sind und in Bezug auf den wirtschaftlichen Erfolg (Rentabilität) eingestuft werden. Durch diese Selektion und Einordnung lässt sich die Aussagefähigkeit in folgenden Punkten verbessern:

- Es kann ermittelt werden, welchen Anteil der IFCN-Betrieb aus der Grundgesamtheit abbildet.

- Der IFCN-Betrieb kann bezüglich seiner Rentabilität eingeordnet werden (wichtig für die Interpretation der IFCN-Ergebnisse). Dies ermöglicht eine differenziertere Betrachtung der Simulationsergebnisse typischer IFCN-Betriebe (insbesondere im Hinblick auf kurz-, mittel- und langfristige Einkommenseffekte).

Wie im Ergebniskapitel 6.4 gezeigt werden kann, stößt das bestehende Auswahlverfahren der IFCN-Betriebe auf Grenzen. Deshalb wird abschließend ein Verfahren vorgestellt, wie künftig die Eckdaten zur Auswahl typischer IFCN-Betriebe direkt aus dem FADN generiert werden können.

\subsection{Auswahl der FADN-Vergleichsgruppe}

Um die Betriebsdaten besser vergleichen und einordnen zu können, sind bei der Auswahl der Vergleichsbetriebe insbesondere folgende Fragen zu klären:

- Regionale Eingrenzung der FADN-Stichprobe: Es ist zunächst festzulegen, welche Regionen zur Auswahl ähnlicher FADN-Betriebe berücksichtigt werden sollen. Einerseits liegt es aufgrund ähnlicher Standortbedingungen nahe, diejenigen Regionen einzubeziehen, in denen der IFCN-Betrieb lokalisiert ist. Andererseits ist die Wahrscheinlichkeit, Betriebe zu finden, die dem typischen IFCN-Betrieb ähnlich sind, umso größer, je mehr Regionen einbezogen werden. 
- „Abschneidegrenze“: es ist eine Entscheidung zu treffen, bis zu welchem euklidischen Distanzmaß FADN-Betriebe in die Vergleichsgruppe aufgenommen werden sollen. Bei der Auswahl der Abschneidegrenze sind folgende Aspekte zu berücksichtigen:

- Die Anweisung der EU-Kommission, die besagt, dass aus Datenschutzgründen FADN-Betriebsdaten erst ab einer Mindestanzahl von 15 Betrieben je Gruppe veröffentlicht werden dürfen.

- Repräsentativität: Durch die FADN-Vergleichsgruppe soll ein möglichst großer Anteil Betriebe aus der Grundgesamtheit abgebildet werden. Dieser Anteil nimmt in der Regel mit steigender Anzahl von FADN-Betrieben in der Vergleichsgruppe $\mathrm{zu}$.

- Selektionsgenauigkeit: Je mehr Betriebe in die Vergleichsgruppe aufgenommen werden, umso eher besteht die Gefahr, dass die durchschnittliche Ähnlichkeit und damit auch die Vergleichbarkeit der FADN-Betriebsgruppe zum typischen Betrieb abnimmt.

Diese und weitere methodische Aspekte zum gewählten Selektionsverfahren mittels euklidischer Distanz werden im folgenden Kapitel 6.2.1 erläutert.

\subsubsection{Selektion der Vergleichsgruppe mittels euklidischer Distanz}

Um die Datensätze FADN und IFCN besser vergleichen und anschließend verknüpfen zu können, ist es erforderlich, die ausgewählten typischen IFCN-Betriebe einer möglichst ähnlichen Vergleichsbasis aus dem FADN gegenüberzustellen (vgl. FENNECKER, 2002, S. 192; Schulze Pals, 1994, S. 77; Offermann und Nieberg, 2000, S. 5 ff.; OffermanN und NiEBERG, 2001, S. 421-427; JÄGERSBERG, 2002).

In Anlehnung an das multivariate Analyseverfahren der Clusteranalyse wird in der ersten Stufe eine möglichst ähnliche FADN-Vergleichsbasis selektiert. Eine gleichartig strukturierte Vorgehensweise findet sich bei FENNEKER (2002) bezogen auf den Bereich der Schweineproduktion und bei Schulze PALS (1994) im Rahmen der durchgeführten Begleitforschung zu den wirtschaftlichen Folgen der Umstellung auf ökologischen Landbau. 
Zur Auswahl der Vergleichsbetriebe wird in Anlehnung an die Clusteranalyse ${ }^{4}$ unter simultaner Berücksichtigung verschiedener Vergleichsmerkmale für jeden ausgewählten typischen IFCN-Betrieb eine Gruppe von FADN-Vergleichsbetrieben selektiert (Stufe 1). Die Ähnlichkeit zwischen zwei Betrieben wird anhand der euklidischen Distanz ${ }^{5}$ hergeleitet. Die euklidische Distanz stellt ein Ähnlichkeitsmaß zwischen dem typischen Betrieb und jeweils einem Betrieb aus dem FADN-Datenpool dar.

Je niedriger die euklidische Distanz ausfällt, umso höher ist die Ähnlichkeit der FADNBetriebe zum typischen Betrieb. Da die typischen Betriebe nicht konform sind mit der Grundgesamtheit, ist eine Standardisierung der Auswahl von FADN-Betrieben nicht möglich. Daher muss eine „ad-hoc“ Entscheidung über die Anzahl ähnlicher FADN-Betriebe getroffen werden, die in die Vergleichsgruppe aufzunehmen sind. Unter Berücksichtigung unterschiedlicher Gesichtspunkte (Repräsentativität, Selektionsgenauigkeit, vergleichbare Gruppen) wird die Anzahl FADN-Vergleichsbetriebe in dieser Arbeit auf 25 begrenzt (vgl. FENNEKER, 2002, S. 194).

Zur Auswahl der Vergleichsbetriebe werden in der ersten Stufe die für die Milchproduktion wichtigsten Kennzahlen (TiETJEN und LANGBEHN, 2000) ${ }^{6}$ des insgesamt ca. 1.900 Variablen umfassenden FADN-Datensatzes herangezogen, die im Folgenden aufgeführt sind:

$4 \quad$ Der Begriff „Cluster“ stammt aus dem Englischen und wird erklärt als: „close group or bunch of similar people, animal or things brought or growing together”. Allgemein versteht man unter Clusteranalyse ein Verfahren zur Klassifikation von Objekten. Die Objekte werden anhand ihrer Merkmalsausprägungen zu Gruppen (Clustern) zusammenfasst, so dass die Objekte in diesen Gruppen sich bezüglich der Merkmalsausprägungen möglichst ähnlich sind, die Gruppen untereinander sich aber möglichst deutlich voneinander unterscheiden. Es können dabei grundsätzlich beliebig viele Merkmale der Objekte in die Analyse einbezogen werden, so dass die Clusteranalyse den multivariaten Verfahren zuzurechnen ist (OERTHEL und TUSCHL, 1995). Wichtig für das Ergebnis einer Clusteranalyse sind daher die Bestimmung der Ähnlichkeit von Objekten bzw. Clustern und das Kriterium, mit dem man eine möglichst gute Trennung der Cluster erreichen will (BORTZ, 1993). Die Clusteranalyse ist somit als exploratives Verfahren ein Hilfsmittel, um eine Fülle von Daten, die die Untersuchungsobjekte beschreiben, transparent zu machen (vgl. STEINHAUSEN und LANGER, 1977; SPÄTH, 1983; BoRTZ, 1989; SCHULZE PALS, 1994, S. 88 ff.).

Proximitätsmaß: Die Ähnlichkeit (Proximität) von zwei Objekten lässt sich sowohl durch ein Ähnlichkeitsmaß als auch durch ein Distanzmaß ausdrücken (im zweiten Fall wird die Unähnlichkeit zweier Objekte gemessen). Die beiden ähnlichsten Objekte unter einer Vielzahl von Objekten sind im ersten Fall die, bei denen ein Ähnlichkeitsmaß den größten Wert annimmt und im zweiten Fall die, bei denen ein Distanzmaß den kleinsten Wert annimmt. Beide Arten der Ähnlichkeitsmessung sind somit äquivalent. Ob Ähnlichkeits- oder Distanzmaße angewendet werden hängt meistens auch von der Skala ab, auf der die Merkmalsausprägungen der Objekte gemessen werden. Es bieten sich z. B. bei quantitativen Merkmalen - aufgrund ihrer in diesem Fall leichten Berechenbarkeit - Distanzmaße an (OERTHEL und TuSCHL, 1995).

6 Die von TietJen und LANGBEHN (2000) durchgeführten Korrelationsrechnungen zur Messung der Bedeutung verschiedener abhängiger Variablen (z. B. Bestandsgröße und Milchleistung) unterstreichen diese Auswahl. 
- Bundesland

- Betriebstyp (hier: spezialisiert auf Milchproduktion)

- Milchviehbesatz (LU bzw. RGV/Betrieb)

- Milchproduktion (dt Milch/Betrieb)

- Landwirtschaftliche Nutzfläche (ha LF/Betrieb)

- Grünland (ha/Betrieb)

- Arbeitskräftebesatz insgesamt

Es werden sowohl Variablen berücksichtigt, die den Gesamtbetrieb charakterisieren als auch Kennzahlen, die sich auf den Betriebszweig Milch beziehen (vgl. Schulze PALS, 1994, S. 86; BACKHAus et al., 1996, S. 261 ff.). „Mit steigender Anzahl von Vergleichsmerkmalen kann die Abweichung zwischen einzelnen Variablen jedoch zunehmen“ (FENNEKER, 2002, S. 194). Daher werden in der ersten Stufe lediglich einige wenige vorwiegend strukturelle Kennzahlen ${ }^{7}$ zur Identifizierung geeigneter Vergleichsbetriebe herangezogen, die jedoch indirekt ${ }^{8}$ auch Auskunft über die Naturalleistung ${ }^{9}$ (in diesem Fall: die Milchleistung) geben. Die Variablen zum Besatz von Lohn- und Familienarbeitskräften werden zu einer Variablen zusammengefasst, da in den meisten Untersuchungsregionen kaum Lohnarbeitskräfte vorhanden sind.

Da sich die Größenordnungen der einzelnen Kennzahlen wesentlich voneinander unterscheiden können und damit auch unterschiedlich großen Einfluss auf die euklidische Distanz besitzen, werden die Daten vor der Berechnung zunächst anhand einer z-Transformation standardisiert. Dabei wird die jeweilige Abweichung vom Variablenmittelwert durch die Standardabweichung der Variable dividiert (vgl. BoRTZ, 1995, S. 387).

Die regionale Zuordnung der FADN-Vergleichsgruppe zum untersuchten typischen Betrieb erfolgt im ersten Schritt auf Bundeslandebene. ${ }^{10}$ Das heißt, der typische Betrieb und die FADN-Vergleichsgruppe müssen demselben Bundesland angehören, um ähnliche Standortbedingungen zwischen dem typischen Betrieb und der FADN-Vergleichsgruppe

7 Gemeint sind hier Daten, die die Ausstattung des Betriebes mit Produktionsfaktoren (Arbeit, Boden, Kapital) charakterisieren, als auch die Produktionsstruktur (Bodennutzung, Viehhaltung) kennzeichnen.

$8 \quad$ Über die angegebene Milchproduktion und den Milchviehbesatz: Milchproduktion : Anzahl Milchkühe $=$ Milchleistung.

9 Die Naturalleistung gibt zum Teil auch Hinweise über die Betriebsleiterqualität, wobei berücksichtigt wird, dass schlechte Leistungen auch durch andere vom Betriebsleiter nicht beeinflusste Umwelteinflüsse hervorgerufen werden.

10

Beziehungsweise auf Basis der FADN-Regionen. 
sicherzustellen. ${ }^{11}$ Da die Gesamtähnlichkeit zwischen zwei Betrieben umso größer bzw. die euklidische Distanz umso kleiner ist, je mehr Betriebe vorliegen, aus denen entsprechend ausgewählt werden kann, wird in einigen Fällen (wenn in einem Bundesland nicht genügend ähnliche Betriebe vorhanden sind) im Rahmen von Variationsrechnungen die FADNStichprobe um eine weitere Region mit ähnlichen natürlichen Voraussetzungen erweitert.

Die Ähnlichkeit nimmt mit größerer Anzahl von Betrieben im FADN-,,Auswahlpool“ zu, und die Varianz der Vergleichsvariablen wird in Grenzen gehalten. Dies veranschaulicht beispielhaft die in Tabelle 6.1 aufgeführte Standardabweichung der Vergleichsgruppen zum typischen 68-Kuhbetrieb in Bremervörde/Niedersachsen.

In diesem Beispiel (Tabelle 6.1) wird der FADN-,,Betriebspool“ regional um das Bundesland Schleswig-Holstein erweitert. Dies führt zu einer Verringerung der euklidischen Distanz. Aufgrund der erweiterten Stichprobe bei vergleichbaren natürlichen Standortbedingungen erhöht sich die Wahrscheinlichkeit, Betriebe ähnlicher Betriebsstruktur zu finden. ${ }^{12}$

Tabelle 6.1: $\quad$ Median und Standardabweichung der FADN-Vergleichsgruppe zum typischen 68-Kuhbetrieb in Bremervörde/Niedersachsen bei unterschiedlicher Datenbasis

\begin{tabular}{lrrrr}
\hline Datenbasis & \multicolumn{2}{c}{ D-68 NI } & \multicolumn{2}{c}{ D-68 SHNI } \\
Ähnlichkeitskennzahl & \multicolumn{2}{c}{$\mathbf{1 , 0 3}$} & 0,82 \\
\hline Variable & Median & Standardabweichung & Median & Standardabweichung \\
\hline Landwirtschaftliche Nutzfläche (ha) & 64,44 & 32,47 & 64,91 & 30,98 \\
Grünlandfläche (ha) & 46,18 & 25,61 & 42,41 & 25,07 \\
Anzahl Milchkühe & 55 & 30 & 54 & 28 \\
Milchproduktion (t) & 385,73 & 230,26 & 374,49 & 214,71 \\
Milchleistung (kg) & $6.828,51$ & $1.266,82$ & $6.799,41$ & $1.246,52$ \\
Familienarbeitskrafteinheiten & 1,94 & 1,04 & 1,88 & 0,91 \\
Fremdarbeitskrafteinheiten & 0 & 0 & 0 & 0 \\
\hline
\end{tabular}

Quelle: Eigene Berechnungen mit FADN und IFCN (2003).

11 Die landwirtschaftliche Vergleichszahl (VZ) ist im FADN-Datensatz als Selektionsvariable nicht vorhanden. Sie bezeichnet die mit $\mathrm{Zu}$ - und Abschlägen für die natürlichen Ertragsbedingungen, die betriebsinternen und -externen wirtschaftlichen Ertragsbedingungen sowie für die Grundsteuer und die besonderen Entwässerungskosten versehene Ertragsmesszahl (EMZ) der Reichsbodenschätzung von 1934. 
Die wesentlichen Schritte bei der Auswahl der FADN-Vergleichsbetriebe sind in Abbildung 6.1 nochmals im Überblick dargestellt.

Abbildung 6.1: $\quad$ Vergleich von IFCN-Betrieben mit FADN-Betrieben

\section{Spezialisierte Milchviehbetriebe ( $>60 \%$ Milcherlöse)}

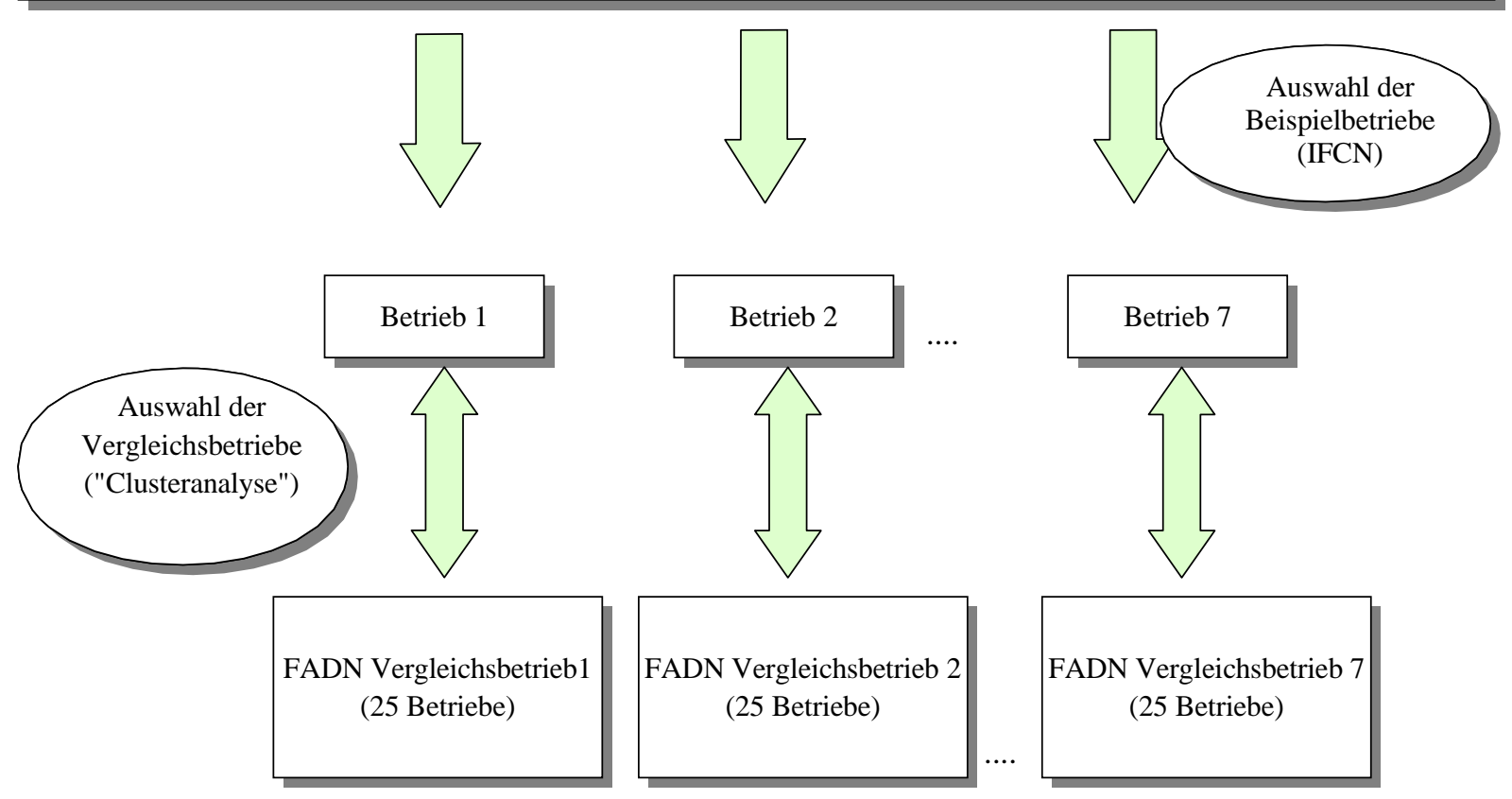

Quelle: Eigene Darstellung in Anlehnung an Fenneker (2002), S. 194.

Diese Vorgehensweise zur Auswahl von Vergleichsbetrieben ist grundsätzlich erweiterbar, beispielsweise indem

- anstelle des euklidischen Distanzmaßes, welches alle Parameter gleichzeitig einbezieht, absolute/relative Abweichungsgrenzen für jedes Kriterium einzeln festgelegt werden können. Damit könnten Abweichungen bei den bedeutendsten Kriterien sehr gering gehalten werden (z. B. bei Milchviehbetrieben: Anzahl Milchkühe, Milchquote) (vgl. NieBERG, OfFERMANn, ZANDER, 2007).

- Erfolgskriterien bei der Selektion von FADN-Vergleichsgruppen zum typischen IFCN-Untersuchungsbetrieb berücksichtigt werden. 


\subsubsection{Statistische Einordnung typischer IFCN-Betriebe in die Vertei- lung der FADN-Betriebsgruppe}

In diesem Kapitel wird die methodische Vorgehensweise bei der Einordnung der IFCNBetriebe in die Verteilung der FADN-Vergleichsbetriebe erläutert. Dabei wird auf folgende Fragestellungen der Reihenfolge nach eingegangen:

- Was ist das (Teil-) Problem?

- Wie sind andere damit umgegangen?

- Wie ist der Entwicklungsstand zu bewerten?

- Wie können Ansätze zusammengeführt und weiterentwickelt werden, um das Problem optimal zu lösen?

\subsubsection{Problemstellung bei der statistischen Einordnung}

Zur Einordnung der regionaltypischen Betriebe ist zu ermitteln, welche Bedeutung die Betriebe in der Region haben und für welche und wie viele Betriebe somit Rückschlüsse der Analyseergebnisse möglich sind. Bei der Beantwortung dieser Art von Fragestellung werden Wissenschaftler häufig mit folgenden Problemen konfrontiert:

- Die Wahl der räumlichen Abgrenzung: mit der Wahl der Datengrundlage ist in der Regel eine räumliche Abgrenzung verbunden. Das Ergebnis der Einordnung ist je nach Größe des Raumes (Land, Region, Gemeinde) unterschiedlich, so dass oft fraglich ist, welche Datengrundlage heranzuziehen ist. Die nicht eindeutige Abgrenzung der jeweiligen Region, die der Betrieb repräsentieren soll, erschwert die Wahl (vgl. GOERTZ, 1998, S. 25 ff.).

- Die geeignete Wahl der Merkmale, anhand derer die Betriebe eingeordnet werden sollen. Die jeweilige Repräsentativität des regionaltypischen Betriebes variiert in Abhängigkeit vom gewählten Merkmal. So kann zum Beispiel ein Betrieb, gemessen an der Kuhzahl, eine Vielzahl von Betrieben einer Region präsentieren; handelt es sich jedoch um einen überdurchschnittlich erfolgreichen Betrieb, ist es möglich, dass dieser Betrieb anhand des Merkmals Gewinn nur selten in dieser Region anzutreffen ist (vgl. GOERTZ, 1998; JÄGERSBERG, 2002).

- Eine Einordnung ist nur anhand der Merkmale bzw. Merkmalskombinationen möglich, die auch für alle betreffenden Betriebe in der Statistik ausgewiesen werden. So ist es derzeit nicht möglich, Betriebe im Hinblick auf das Merkmal „Entwicklungsphase des Betriebes“ einzuordnen, da Angaben über Investitionszeitpunkte in vorhandenen Zahlenwerken nicht vorzufinden sind (GOERTZ, 1998). 
- Die Schichtung in statistischen Publikationen ist meist zu ungenau, um Vergleiche mit regionaltypischen Betrieben zu ziehen (HEMME, 2000, S. 100-103).

- Selbst bei gleichem Merkmal sind die gewählten Statistiken für die verschiedenen Regionen und Länder nicht notwendigerweise vergleichbar, nämlich dann, wenn die jeweiligen Merkmale anders erfasst oder interpretiert werden (vgl. HEMME, 2000; JÄGERSBERG, 2002).

- Die Buchführungsstatistiken sind zum Teil aus steuerlichen Gründen „modifiziert“, um den Gewinn zu reduzieren (z. B. Sonderabschreibungen). Daher ist es möglich, dass die Leistungsfähigkeit der Betriebe in den Statistiken unterschätzt wird, was die Einordnung der typischen Betriebe erschwert (HEMME, 2000).

- Bei Vergleichen mit Offizialstatistiken, die aus steuerlichen Buchführungsabschlüssen resultieren, wird die Vergleichbarkeit ferner dadurch erschwert, dass der Wert der Abschreibung für Gebäude und Maschinen häufig aus den historischen Anschaffungskosten abgeleitet wird (AMELUNG, 1999, S. 90).

- Eine Vielzahl der Buchführungsstatistiken erscheint in zeitlicher Verzögerung von bis zu einem Jahr und später nach Abschluss des Wirtschaftsjahres. Dadurch wird die Einordnung aktuell erhobener Betriebe ebenso erschwert (HEMME, 2000; JÄGERSBERG, 2002).

Die genannten Probleme verdeutlichen, wie schwierig die Einordnung der typischen Betriebe tatsächlich ist. Dennoch ist es möglich, zumindest einen Einblick zu vermitteln, welche Bedeutung die gewählten Betriebe in dem jeweiligen Land oder der Region haben.

\subsubsection{Herangehensweise anderer Wissenschaftler}

In diesem Kapitel wird zusammengefasst, wie andere Wissenschaftler mit der Einordnung der Analyseergebnisse in statistisches Datenmaterial umgegangen sind. Von den Studien, die sich mit der statistischen Einordnung beschäftigt haben (COLSON, CHATELLIER, FuENTES, 1999; Fraysse, 2002 Perrot et al., 2002; Schulze PAls, 1994; JäGersBerg, 2002), werden in diesem Kapitel nur die Ansätze näher vorgestellt, die der Problemstellung dieser Arbeit am nächsten liegen und am ehesten zu einem geeigneten Lösungsansatz im Rahmen dieser Arbeit beitragen können.

Goertz (1998), Amelung (1999), Christoffers (2001) und HEMme (2000) haben versucht, typische Betriebe in nationale Buchführungsstatistiken und u. a. auch in das nationale Testbetriebsnetz einzuordnen. Bei Goertz, Hemme und Christoffers erfolgte die Einordnung der erhobenen typischen Milchviehbetriebe beispielsweise anhand des Merkmals Betriebsgröße, wobei der Parameter Kuhzahl/Betrieb herangezogen wurde. Neben der nationalen Statistik haben Goertz, Hemme und Christoffers für diese Einordnung auch die 
regionale Betriebsgrößenstatistik herangezogen, da die nationale Statistik Betriebe mit mehr als 200 Kühen in einer Gruppe zusammenfasst, und die Einordnung großer ostdeutscher Betriebe daher auf dieser Datenbasis nicht sinnvoll erfolgen konnte. Zur Einordnung der typischen Betriebe nach Leistungsfähigkeit wurde der Betriebsgewinn je Kuh gewählt. Dies erwies sich deshalb als schwierig, da verschiedene Statistiken zu unterschiedlichen Ergebnissen hinsichtlich der Leistungsfähigkeit von landwirtschaftlichen Betrieben einer Größenklasse gekommen sind. Die Erstellung von Verteilungskurven auf Grundlage des Parameters „Betriebsgewinn“ war mit der vorliegenden Datengrundlage (Buchführungsstatistik, nationale Testbetriebsstatistik, harmonisierte EU-Statistiken/FADN) nicht möglich. Gründe dafür lagen in der begrenzten Differenzierung der Ergebnisse, erheblichen Schwankungen in den Ergebnissen von Jahr zu Jahr, Ergebnisvariablen, die nicht mit TIPI-CALVariablen verglichen werden können sowie in zeitlichen Verzögerungen bei der Erscheinung von Buchführungsstatistiken und der nationalen Testbetriebsstatistik, wodurch die Einordnung der aktuell erhobenen typischen Betriebe weiter erschwert wird. Bei AMELUNG (1999) erfolgte die strukturelle Einordnung extensiver Marktfruchtbaubetriebe in Deutschland und Ungarn anhand des Merkmals Betriebsgröße, gemessen in Hektar (Betriebsgrößenverteilung, prozentualer Anteil an der Ackerfläche). Es wurde auf deutsche Regionalstatistiken und die ungarische Landesstatistik zurückgegriffen. Die Einordnung nach der Leistungsfähigkeit erfolgte anhand des Merkmals Gewinn pro Hektar. In Ungarn erfolgte aufgrund der verhältnismäßig schwachen Datenbasis der relevanten Agrarstatistiken besonders in Bezug auf die Leistungsfähigkeit zusätzlich eine Expertenbefragung zu den ausgewählten Merkmalen. Auch bei AMELung ermöglichte die Einordnung der Panelbetriebe keine belastbaren Aussagen im Hinblick auf die Repräsentativität der Betriebe in den Ländern und Regionen. Sie sind jedoch wie bei Goertz, Hemme und ChristOFFERs im Rahmen des zugrundeliegenden Erhebungsprozesses als „typisch“ in Faktorausstattung, Aufwendungen und Erträgen für die mittlere bzw. obere Betriebsgrößenklasse identifiziert worden.

In Frankreich wurden Lösungswege gesucht, um die Daten aus dem Netzwerk der sogenannten „cas types“ mit dem französischen Testbetriebsnetz bzw. dem FADN zu verknüpfen. Um die Diversität europäischer Weidehaltungssysteme besser verstehen und in Bezug auf Auswirkungen von Politikänderungen besser beurteilen zu können, wurde im Auftrag der EU-Kommission von CHATELLIER et al. $(1997,2000)$ eine Typologie auf Basis der zur Verfügung stehenden europäischen FADN-Daten entwickelt (vgl. CHATELLIER et al., 1997, 2000). Diese Typologie - GLS (Grazing Livestock Systems) genannt - findet nun auch bei der Generaldirektion für Landwirtschaft der Europäischen Kommission Verwendung. Die europäischen Weidehaltungssysteme wurden in Abhängigkeit von der geographischen Lage, der Futterbasis und der Produktionstechnik einheitlich auf EU-Ebene in vier Produktionsklassen, in neun Viehhaltungssysteme und 18 technische Systeme untergliedert. Diese 
Typologie, die komplementär ist zu dem Ansatz nach Betriebstypen ${ }^{13}$, ermöglicht vergleichende Studien der Strukturen und ökonomischen Ergebnisse von landwirtschaftlichen Betrieben mit vergleichbaren Produktionssystemen in den EU-Ländern bzw. unterschiedlichen Regionen in der EU. Obwohl die GLS-Typologie die Komplexität europäischer Tierproduktionssysteme und die Heterogenität der Betriebsstrukturen (ökonomisch Dimension, Spezialisierung, Intensivierung) aufzeigt, bestehen gewisse Einschränkungen in Bezug auf die derzeit fehlende Präzisierung verschiedener Variablen im FADN, um die unterschiedlichen Formen der Milch-, Rind-, Schaf- und Ziegenproduktion etc. zu untersuchen.

Daher wird diese Methode der Klassifizierung in Frankreich ergänzt durch den Verschnitt mit Daten aus sogenannten „cas typiques“ („cas types“), die weitere technische, ökonomische oder agrarpolitische Indikatoren liefern (CHATELLIER et al., 2000, S. 212). Von den Landwirtschaftskammern (CA) ${ }^{14}$ und dem Institut für Rinderhaltung (Institut de l' Elevage) werden diese „cas typiques“ „(cas types“) u. a. auch der Milchviehhaltung erhoben. Diese Daten erfüllen zwar nicht das Repräsentativitätskriterium, haben gegenüber den FADN-Daten jedoch den Vorteil, dass sie tiefer regionalisiert sind und neben den Angaben zur Wirtschaftlichkeit der Systeme eine Fülle produktionstechnischer Details enthalten (vgl. Perrot, 2002; Deblitz, 1993, S. 188). Sie werden aus Buchführungsdaten (französische Testbetriebsdaten = RICA) einer Gruppe von Betrieben gewonnen, die sich „Erhebung der zukunftsorientierten Rinderzüchter“ (EDB) nennt. Die teilnehmenden Betriebe zeichnen sich aus durch überdurchschnittlich gute wirtschaftliche und produktionstechnische Leistungen. Die „cas typiques“ entstehen, indem die französischen Testbetriebsdaten mit Beratern und Landwirten adjustiert werden. Mit Hilfe der Erhebungsergebnisse dieser Betriebe werden Faltblattsammlungen für verschiedene Betriebstypen, die sogenannten „cas types““ ${ }^{15}$ erstellt. Sie dienen Planungs- und Beratungszwecken. Die „cas types“ sollen Junglandwirten sowie Betrieben mit Anpassungsproblemen und der Beratung als Orientierungshilfe zur Verfügung stehen. Die Landwirte können ihre eigenen Betriebe mit den sogenannten „cas typiques“ vergleichen. Es sind darin 17 „cas types“ für Milchviehhalter, die sich hinsichtlich ihrer Faktorausstattung und der natürlichen Voraussetzungen der Betriebe unterscheiden.

13 Nach der OTEX-Typologie: Definition der Hauptproduktionszweige eines Betriebes auf Basis ihres Anteils am betrieblichen Gesamtdeckungsbeitrag (vgl. Kapitel 4.2/FADN).

14 Chambre d’Agriculture.

15 Die Faltblätter enthalten einen Betriebsspiegel, ein Schema zum Herden- und Weidenmanagement, Angaben über Ernte- und Lagermenge sowie über Gebäude- und Maschinenausstattung als auch einen Kalender mit saisonalen Arbeitsspitzen. Ein beigefügtes loses Blatt enthält Informationen zu den ökonomischen Größen, das jährlich aktualisiert wird (vgl. DEBLITZ, 1993, S. 188). 
Dieser Verschnitt der „cas types“ mit FADN-Daten ist möglich, da die französischen „cas types“ den Struktur- und Leistungsmerkmalen aus der GLS-Typologie weitgehend folgen (vgl. Perrot et al., 2002). Die Eigenschaften der 17 bedeutendsten Betriebstypen laut GLS-Typologie sind im Anhang in Tabelle A6.1 dargestellt.

\subsubsection{Bewertung des Entwicklungsstandes}

Die vorgestellte Einordnung von Panelbetrieben (regionaltypische Betriebe) in die Grundgesamtheit aller Betriebe einer Region weist Schwachstellen auf. Die Einordnung erlaubt keine belastbaren Aussagen im Hinblick auf die Repräsentativität der Betriebe in den Ländern und Regionen. Bei der Einordnung gemäß der Struktur tauchen insbesondere in den Untersuchungsregionen der neuen Bundesländer Schwierigkeiten auf, da die Betriebsgrößenstatistik die Betriebsgrößenklassen der neuen Bundesländer widerspiegelt und nicht die der spezifischen Untersuchungsregion (z. B. Sachsen-Anhalt). Die Aussagekraft der Buchführungsstatistiken zur Abschätzung der Leistungsfähigkeit der Panelbetriebe im Vergleich zu Betrieben einer Region ist nicht vollständig gegeben, weil verschiedene Buchführungsstatistiken zu unterschiedlichen Ergebnissen hinsichtlich der Leistungsfähigkeit von landwirtschaftlichen Betrieben einer Größenklasse kommen (HEMME, 2000, S. 102; CHRISTOFFERS, 2001, S. 27).

Bezogen auf das französische Beispiel stellt sich die Frage nach der Vergleichbarkeit des französischen Netzwerkes der „Cas-Types“ mit dem internationalen Netzwerk Typischer Betriebe aus dem IFCN. Beide Konzepte sind ähnlich, wobei die französischen „CasTypes “ stärker auf produktionstechnische Analysen ausgerichtet sind, während bei den typischen IFCN-Betrieben der Schwerpunkt auf ökonomischen Analysen und Wettbewerbsvergleichen liegt. ${ }^{16}$ Aufgrund des unterschiedlichen Erhebungskonzeptes ist auch die Zuordnung typischer Betriebe in die GLS-Typologie weniger eindeutig.

\subsubsection{Eigene Lösungsansätze}

Aufbauend auf den zuvor beschriebenen Erfahrungen wird in diesem Kapitel untersucht, wie im Rahmen der statistischen Einordnung zwei verschiedene Ansätze zusammengebracht und weiterentwickelt werden können, um die oben beschriebenen Probleme etwas zu entschärfen.

16 Aufgrund unterschiedlicher Erfassung und Interpretation der Merkmale der „Cas-Types“ wurde ein Modul zur Übertragung der Daten ausgewählter französischer „Cas-Types“ in das Netzwerk typischer IFCN-Betriebe erstellt (JÄGERSBERG und PERROT, 2001). Dies ermöglicht somit auch den internationalen Vergleich der französischen „Cas-Types“. 
Dabei erfolgt zunächst die Auswahl geeigneter Indikatoren, anhand derer die Betriebe eingeordnet werden sollen. In Kapitel 6.3 wird anschließend dargestellt, in welcher Weise die FADN-Daten zur Einordnung der typischen Betriebe aufbereitet werden, um den Schwierigkeiten unterschiedlicher Ansätze bei der Einordnung bestmöglich zu begegnen.

\section{Auswahl geeigneter Parameter zur Einordnung typischer IFCN-Betriebe}

Der gewählte Indikator zur Einordnung typischer IFCN-Betriebe in die kumulierte Verteilung der FADN-Vergleichsbetriebe sollte im Idealfall folgenden Anforderungen genügen:

- Der Indikator sollte in Einklang mit dem Ziel dieser Arbeit stehen - der verbesserten Abbildung einzelbetrieblicher Einkommens- und Anpassungseffekte bei verschiedenen Politiken.

- Er sollte sowohl im IFCN- als auch im FADN-Datensatz vorhanden sein.

- Die Erfassung und Berechnung des Indikators sollte bei beiden Datensätzen möglichst ähnlich sein, um Interpretationsprobleme zu vermeiden.

- Der Indikator sollte bei beiden Datensätzen für dasselbe Wirtschaftsjahr vorhanden sein.

Wegen der vorher beschriebenen Schwierigkeiten beim Vergleich aktuell erhobener einzelbetrieblicher Daten mit Buchführungsstatistiken konnte kein Indikator gefunden werden, der sowohl dem Ziel dieser Arbeit entspricht, als auch auf Basis ähnlicher Definition in beiden Datensätzen vergleichbar ist und möglichst noch einen Großteil der Betriebe einer Region abbildet. Deshalb wurde die Entscheidung getroffen, die Produktionskosten heranzuziehen. Die Produktionskosten wurden in dieser Arbeit deshalb gewählt, weil sie wichtige Hinweise dafür geben, wohin die Milchproduktion potenziell bei zunehmender Liberalisierung des Milchmarktes wandern wird. Dabei werden zwei Kostenarten unterschieden:

a) Vollkosten: zur Abbildung langfristiger Einkommenseffekte;

b) Kosten aus der Gewinn-und-Verlust-Rechnung: zur Abbildung kurz-/mittelfristiger Einkommenseffekte (vgl. ZEDDIES, 1991, S. 75-87).

Da die FADN-Daten im Vergleich zu den IFCN-Datensätzen nur gesamtbetrieblich vorliegen und kalkulatorische Größen nur teilweise berücksichtigt sind, treten auch bei diesem Indikator Probleme der Vergleichbarkeit auf. Die Methoden zur Ermittlung der Produktionskosten der IFCN- und FADN-Daten werden daher einander so weit wie möglich angepasst. Die Auswertungsschritte zur Aufbereitung des Datenmaterials werden im Kapitel 6.3 beschrieben. 


\subsection{Aufbereitung des FADN-Datenmaterials zur statistischen Ein- ordnung der typischen Betriebe}

\subsubsection{Berechnung der FADN-Produktionskosten in Anlehnung an die IFCN-Methodik}

Bei der statistischen Einordnung typischer Betriebe in die Verteilung der FADN-Betriebe (auf Basis der Kostenindikatoren) erfolgt die Berechnung der Produktionskosten des Betriebszweiges Milcherzeugung bei den ausgewählten FADN-Betrieben in Anlehnung an die Methoden der IFCN-Kostenanalysen. Die FADN-Daten müssen für die Produktionskostenschätzung aufbereitet werden, da im Betriebsbogen des FADN nicht vorgesehen ist, alle mit der Milcherzeugung verbundenen Kosten getrennt aufzuführen. Dazu wird ein von GoERTz (Goertz, 1999) entwickeltes Modell für die Aufteilung der Kosten verwendet. Dieses Modell verwendet als Datengrundlage nationale Testbetriebsdaten und ist für die Berechnung mit FADN Daten entsprechend aufbereitet worden.

Das von Goertz entwickelte Kostenallokationsmodell hat den Vorteil, dass die Methode der (Voll-) Kostenanalyse und Kostenallokation in Anlehnung an die Methoden der IFCNKostenanalysen erfolgt. Dennoch verbleiben einige Unterschiede zwischen beiden Bewertungsansätzen, die im Zuge dieser Arbeit gelöst werden bzw. bei der Interpretation der Ergebnisse zu beachten sind. Das Kostenallokationsmodell von GoERTz weist eine hohe Ähnlichkeit zu dem Kostenallokationsmodell der EU-Kommission für Milch und Rindfleisch auf (EU FADN-DG/Agri, 2007). ${ }^{17}$

Die Produktionskosten im FADN werden soweit wie möglich aus den Aufwandspositionen der Gewinn-und-Verlust-Rechnung (GuV) ermittelt. ${ }^{18}$ Die Produktionskosten sind im FADN größtenteils nur aggregiert auf Ebene des Betriebes oder verschiedener Produktionsbereiche erfasst. Daher werden die erfassten Kosten aus dem FADN für die vorliegende Analyse mit Hilfe verschiedener Allokationsfaktoren soweit wie möglich dem Betriebszweig Milchproduktion zugeteilt. Zur Berechnung der kalkulatorischen Kosten werden die regional üblichen Kostensätze für Boden, Arbeit und Kapital herangezogen. Die Berechnung der Produktionskosten erfolgt auf der Ebene jedes Einzelbetriebs.

$17 \quad$ Nähere Details zum Kostenallokationsmodell für Milch und Rindfleisch der EU-Kommission sind auf folgender Internetseite nachzulesen: http://ec.europa.eu/agriculture/analysis/perspec/index2_en.htm.

18

Die Produktionskosten werden pro Liter Milch ausgewiesen und auf 4 \% Fett korrigiert (vgl. HoLZNER, 2003, S. 109). 
Im IFCN werden die Daten sowohl auf Betriebsebene als auch für einzelne Betriebszweige detailliert im Rahmen des Panelprozesses erhoben (www.ifcnnetwork.org). Dies gilt auch für die variablen Kosten einzelner Betriebszweige und für den Gesamtbetrieb. In Bezug auf die Kosten und Erlöse des Gesamtbetriebes (sowie Subventionen) für gewöhnlich aufgeteilte Aufwendungen und Faktoren wie Gemeinkosten, Abschreibungen, Zinskosten, Arbeitskosten und Landkosten ist eine Zuteilung auf die einzelnen Betriebszweige notwendig. In den meisten Fällen erfolgt die Kostenzuteilung zu den einzelnen Betriebszweigen durch folgende Zuteilungsfaktoren:

- der Anteil des Betriebszweiges an der gesamten landwirtschaftlichen Nutzfläche

- der Anteil des Betriebszweiges an den gesamten Arbeits- und/oder Maschinenstunden

- der Anteil des Betriebszweiges am Bruttogewinn oder Gesamterlös.

Im Anhangstext A 6.1 wird aufgezeigt, wie der FADN-Datensatz anzupassen ist, um ihn zur statistischen Einordnung typischer IFCN-Betriebe verfügbar zu machen. Dazu wird die Aufbereitung des Datenmaterials für die Ermittlung der ausgewählten Kostenindikatoren beschrieben und die Vorgehensweise bei der Schätzung der Produktionskosten (in Anlehnung an die Methoden des Modells TIPI-CAL im Rahmen des IFCN) erläutert. Es wird dargestellt, welche Algorithmen ausgewählt werden, um die Produktionskosten dem Produktionsverfahren Milch zuzuordnen.

Da das von GOERTZ entwickelte Modell verlässliche Ergebnisse ausschließlich mit spezialisierten Betrieben generiert, wird in Kapitel 6.3.2 auf die Stichprobenauswahl eingegangen.

\subsubsection{Methodisches Vorgehen bei der Auswahl der Untersuchungs- stichprobe}

Um verlässliche Analyseergebnisse zu generieren, besteht die zu untersuchende Stichprobe ausschließlich aus spezialisierten Milchviehbetrieben (vgl. IsERMEYer, 1988). Diese Eingrenzung erfolgt, um die betriebszweigspezifische Schätzung der Produktionskosten durchführen zu können.

Als Kriterium zur Ermittlung der spezialisierten Milchviehbetriebe wird der Anteil der Milcherlöse (inklusive der Nebenerlöse) an den Gesamterlösen gewählt (vgl. GoERTZ, 1999, S. 2). Um eine ausreichende Anzahl an Betrieben für die Analyse zu erhalten, wird in dieser Arbeit als notwendiger Anteil der Milcherlöse an den Gesamterlösen ein Anteil von $60 \%$ festgelegt. Dies entspricht weitgehend dem gemeinschaftlichen Klassifizie- 
rungssystem der landwirtschaftlichen Betriebe, wonach der Deckungsbeitrag für die Milchkühe zwei Drittel des Deckungsbeitrages des Betriebs übersteigen muss. ${ }^{19}$

Gemäß der Betriebsstrukturerhebung von EUROSTAT (1997) werden auf EU-Ebene 72 \% aller Milchkühe in spezialisierten Milchviehbetrieben gehalten. Dieser Wert schwankt deutlich zwischen den einzelnen Mitgliedstaaten (vgl. Tabelle 6.2). Obwohl die spezialisierten Milchviehbetriebe (Bezeichnung TF41) nicht in allen Mitgliedstaaten als repräsentativ für die Gesamtheit der Milchviehbetriebe gelten können, so wird aber der bedeutendste Anteil der erfassten Milch in spezialisierten Milchviehbetrieben erzeugt (vgl. Chatellier, PfLimlin, 2007).

Tabelle 6.2: $\quad$ Verteilung der Milchkühe gemäß der Betriebstypen (\%)

\begin{tabular}{lcccc}
\hline & TF41 & TF41+TF81 & TF41+TF81+TF43 & TF41+TF81+TF43+TF71 \\
\hline Belgien & 63 & 74 & 88 & 92 \\
Dänemark & 83 & 91 & 92 & 98 \\
Deutschland & 67 & 86 & 90 & 95 \\
Griechenland & 32 & 59 & 71 & 77 \\
Spanien & 80 & 87 & 90 & 93 \\
Frankreich & 57 & 76 & 88 & 93 \\
Irland & 91 & 92 & 98 & 98 \\
Italien & 67 & 80 & 86 & 90 \\
Luxemburg & 82 & 86 & 97 & 99 \\
Niederlande & 91 & 92 & 94 & 94 \\
Österreich & 68 & 73 & 91 & 95 \\
Portugal & 71 & 76 & 82 & 89 \\
Finnland & 87 & 89 & 98 & 99 \\
Schweden & 92 & 96 & 98 & 99 \\
Vereinigtes Königreich & 83 & 93 & 95 & 96 \\
EU & $\mathbf{7 2}$ & $\mathbf{8 4}$ & $\mathbf{9 1}$ & $\mathbf{9 4}$ \\
\hline
\end{tabular}

1) TF81: "field crops and grazing" -Feldfrüchte und Weidehaltung. TF43: "cattle rearing and milk" -Rinderzucht und Milch.

TF71: "mixed livestock maily grazing" -Gemischter Viehbestand - hauptsächlich Weidehaltung.

TF41: "specialzed milk" Spezialisierte Milchviehhaltung.

Quelle: EUROSTAT Farm Structure Survey (1997).

19 Auf diese Weise wurden für das Wirtschaftsjahr 1999/2000 ca. 4.520 Milchwirtschaftsbetriebe aus insgesamt 60.000 FADN-Betrieben in der EU-15 selektiert. 
Aufgrund der Vorgabe der EU, dass veröffentlichte FADN-Durchschnittsergebnisse auf einer Mindestzahl von 15 Betrieben pro Stichprobe basieren müssen und der Tatsache, dass für die Wirtschaftsjahre 1997/1998 und 1998/1999 nur identische Gruppen mit weniger als 15 Betrieben vorliegen, kann nur ein Wirtschaftsjahr berücksichtigt werden.

Zu Kontrollzwecken ${ }^{20}$ wurden die Gruppen mit weniger als 15 identischen Betrieben aus 1997/1998 und 1998/1999 dahingehend überprüft, ob das ausgewählte Untersuchungsjahr deutlich von den Vorjahren abweicht. Dabei zeigte sich, dass die Abweichungen der Betriebe aus den Vorjahren nicht groß sind. Daher wird der Fehler als gering erachtet, wenn Daten nur eines Wirtschaftsjahres berücksichtigt werden.

\subsection{Analyse der Verknüpfung der FADN- und IFCN-Datensätze zur Einordnung der Betriebe}

In diesem Kapitel soll der Frage nachgegangen werden, wie leistungsfähig der entwickelte Verknüpfungsansatz I zur Integration von FADN- und IFCN-Daten ist. Die Leistungsfähigkeit des entwickelten Ansatzes wird am Beispiel der Produktionskosten Milch und der EU-Milchmarktpolitik überprüft. Zunächst werden in Kapitel 6.4.1 geeignete Untersuchungsregionen und -betriebe aus dem IFCN-Netzwerk ermittelt. Anschließend wird die Ähnlichkeit der berücksichtigten FADN-Betriebe mit den typischen Untersuchungsbetrieben aus dem IFCN-Netzwerk analysiert. Es wird der Frage nachgegangen, wie typisch die IFCN-Betriebe für ihre Region und Größenklasse sind und welchen Anteil Betriebe sie aus der Grundgesamtheit der Milchviehbetriebe widerspiegeln. Für die Einordnung der FADN- und IFCN-Datensätze werden zwei Gruppen von FADN-Betrieben mit einer hohen Ähnlichkeit zu den entsprechenden IFCN-Betrieben ausgewählt. Anhand der gewählten FADN-Vergleichsbetriebe werden die Betriebsergebnisse aus dem FADN und IFCN verglichen und eingeordnet. Es wird gezeigt, wie die ermittelten Ergebnisse bei der Interpretation von Analysen zur Agenda-Zwischenbewertung genutzt werden können. Anschließend werden die Verknüpfungsergebnisse diskutiert und Schlussfolgerungen für zukünftige Analysen gezogen. 


\subsubsection{Wahl der Untersuchungsregionen und -betriebe}

In Kapitel 2 wurde die Bedeutung und Struktur der europäischen Milchwirtschaft erläutert. Im Folgenden wird geklärt, für welche Regionen und Betriebe die empirische Untersuchung konkret durchgeführt werden soll.

Um die Verknüpfungsmethoden empirisch überprüfen zu können, sind bei der Wahl der Untersuchungsregionen folgende Anforderungen zu erfüllen:

- $\quad$ hohe Bedeutung der Milcherzeugung für die regionale landwirtschaftliche Produktion,

- breites Betriebsgrößenspektrum,

- ausreichende Datenbasis (FADN- und IFCN-Daten).

Die Auswahl der Untersuchungsregionen und -betriebe basiert maßgeblich auf Daten von EUROSTAT, FAO, ZMP und IFM, veröffentlicht im IFCN Dairy Report, und auf den europäischen Testbetriebsdaten (FADN), die in einer EU-Milchmarktstudie von CHATELLIER und JACQUERIE (2003) ausführlich analysiert worden sind.

\section{Regionale Bedeutung der Milcherzeugung}

Wie der Abbildung 6.2 entnommen werden kann, zählen zu den Regionen ${ }^{21}$, in denen Betriebe mit Milchvieh über 50 \% der regionalen landwirtschaftlichen Erzeugung generieren, Ostdeutschland, Irland, Süddeutschland/Bayern, Nordspanien, Westfrankreich, Ostfrankreich und Schweden. Bei der Interpretation ist zu berücksichtigen, dass bei der Berechnung des Anteils alle Betriebe mit Milcherzeugung berücksichtigt worden sind. ${ }^{22}$ Günsti-

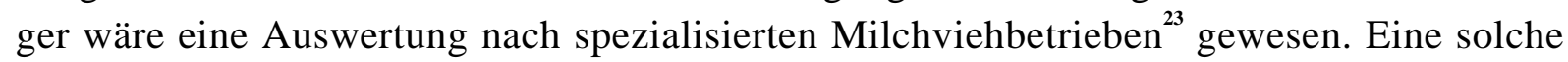
Auswertung ist in der Studie von Vincent Châtellier (ChATEllier und JACQUERIE, 2003) jedoch nicht vorgenommen worden.

21 Bezug nehmend auf die Studie von CHATELLIER und JACQUERIE (2003, S. 10-11) sind aus den 102 FADN-Regionen 27 europäische Produktionsgebiete zusammengefasst worden. Die zugrunde liegende Methodik kann bei CHATELLIER und JACQUERIE (2003) auf S. 10-11 nachgelesen werden. 
Abbildung 6.2: $\quad$ Anteil der milchproduzierenden Betriebe an der monetär bewerteten landwirtschaftlichen Erzeugung (für 27 landwirtschaftliche Produktionsgebiete in 1999/2000)

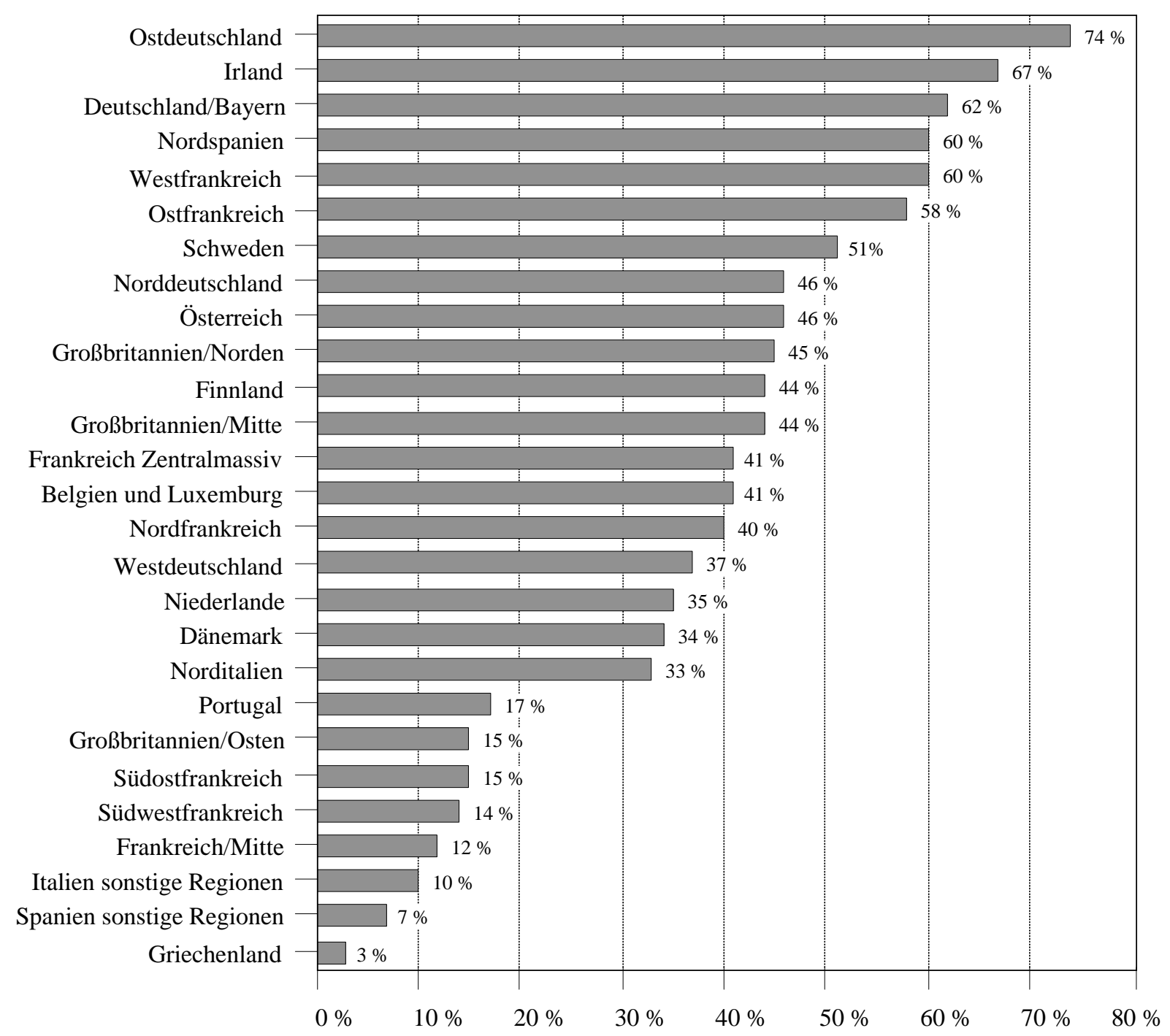

Quelle: Eigene Darstellung und Übersetzung in Anlehnung an Chatellier V., Jacquerie V. (2003): Les expolitations laitieres dans l’Union europeene. Rapport final, April (2003); FADN EU (1999), Europäische Kommission, DG AGRI-A3 / INRA-ESR Nantes.

\section{Betriebsgrößenspektrum}

Die europäischen Milchviehbetriebe sind charakterisiert durch ein breites Betriebsgrößenspektrum. In Großbritannien, Irland, den Niederlanden und in Dänemark wird Milch vorwiegend in vergleichsweise großen Betrieben produziert. In Österreich und Finnland hingegen dominiert die Milchproduktion in kleinen Betrieben (vgl. IFCN Dairy Report 2003, S. 52). 
Dieses breite Betriebsgrößenspektrum spiegelt sich in den europäischen Testbetriebsdaten (FADN) wider. Tabelle 6.3 stellt die Bandbreite der Milchviehbetriebsgrößen innerhalb der 27 Produktionsregionen dar (CHATELLIER und JACQUERIE, 2003, S. 56).

Tabelle 6.3: Durchschnittliche Bestandsgrößen in den europäischen Milchviehherden (gemessen in Rindergroßvieheinheiten)

\begin{tabular}{|c|c|c|c|c|c|}
\hline & $\begin{array}{l}\text { Raufutter- } \\
\text { fresser }\end{array}$ & Rinder & Milchkühe & Mutterkühe & $\begin{array}{c}\text { männliche } \\
\text { Rinder 1-2 Jahre }\end{array}$ \\
\hline Finnland & 28 & 28 & 17 & 0,1 & 1,5 \\
\hline Schweden & 59 & 59 & 32 & 0,5 & 4,3 \\
\hline Österreich & 24 & 24 & 13 & 0,6 & 1,6 \\
\hline Irland & 80 & 77 & 36 & 3,8 & 12,8 \\
\hline Großbritannien & 148 & 134 & 80 & 2,4 & 8,5 \\
\hline Norden & 142 & 126 & 70 & 3,9 & 11,5 \\
\hline Mitte & 148 & 133 & 83 & 1,7 & 6,8 \\
\hline Osten & 166 & 160 & 102 & 1,1 & 7,0 \\
\hline Dänemark & 102 & 102 & 58 & 0,7 & 1,8 \\
\hline Niederlande & 84 & 83 & 54 & 2,0 & 1,5 \\
\hline Belgien u. Luxemburg & 80 & 80 & 37 & 10,2 & 4,2 \\
\hline Deutschland & 65 & 65 & 35 & 0,5 & 4,9 \\
\hline Norden & 81 & 80 & 38 & 0,5 & 9,0 \\
\hline Osten & 350 & 346 & 194 & 2,8 & 27,0 \\
\hline Westen & 52 & 51 & 28 & 0,5 & 4,1 \\
\hline Süden (Bayern) & 45 & 44 & 25 & 0,2 & 1,8 \\
\hline Frankreich & 69 & 68 & 35 & 4,5 & 4,7 \\
\hline Norden & 75 & 75 & 36 & 3,0 & 6,9 \\
\hline Mitte & 93 & 92 & 40 & 8,0 & 9,0 \\
\hline Westen & 68 & 68 & 35 & 4,1 & 5,3 \\
\hline Osten & 84 & 83 & 39 & 4,5 & 7,7 \\
\hline Zentralmassiv & 62 & 59 & 30 & 9,3 & 0,7 \\
\hline Südwesten & 57 & 55 & 33 & 4,0 & 1,1 \\
\hline Südosten & 50 & 48 & 29 & 2,2 & 0,9 \\
\hline Italien & 43 & 42 & 27 & 1,1 & 1,4 \\
\hline Norden & 49 & 49 & 31 & 1,1 & 1,3 \\
\hline übrige Regionen & 31 & 29 & 19 & 0,9 & 1,6 \\
\hline Spanien & 32 & 31 & 22 & 1,1 & 0,0 \\
\hline Norden & 26 & 26 & 19 & 1,2 & 0,0 \\
\hline übrige Regionen & 50 & 45 & 35 & 0,6 & 0,0 \\
\hline Portugal & 29 & 29 & 20 & 0,5 & 0,6 \\
\hline Griechenland & 23 & 22 & 15 & 0,0 & 1,9 \\
\hline Europäische Union & 62 & 61 & 34 & 2,1 & 4,0 \\
\hline
\end{tabular}

Quelle: Eigene Darstellung und Übersetzung in Anlehnung an Chatellier V., Jacquerie V. (2003): Les expolitations laitieres dans l’Union europeene. Rapport final, April (2003); FADN EU (1999), Europäische Kommission, DG AGRI-A3 / INRA-ESR Nantes. 
Die an die empirische Untersuchung dieser Arbeit gestellte Anforderung, dass Regionen einbezogen werden sollen, die unterschiedliche Betriebsstrukturen widerspiegeln und ein breites Betriebsgrößenspektrum abdecken, wird insbesondere in deutschen, britischen und spanischen Produktionsregionen erfüllt.

\section{Datenbasis}

Für die empirische Analyse ist ein möglichst umfassender FADN- und IFCN-Datensatz für dieselben Untersuchungsregionen und denselben Untersuchungszeitraum erforderlich.

$\mathrm{Zu}$ Beginn der Analysen konnte bereits auf ein bestehendes Netz typischer IFCNMilchviehbetriebe in Deutschland, im Vereinigten Königreich (Wales und Nordirland) und in Schweden zurückgegriffen werden. Im Zuge des EU-Projektes ELPEN $^{24}$ wurde das Netzwerk der typischen IFCN-Milchviehbetriebe in Europa ausgeweitet. ${ }^{25}$ Dadurch ist eine Datenbasis von 28 Betriebstypen in neun EU-Ländern (2001) entstanden. In der folgenden Karte 6.1 ist das IFCN-Netzwerk typischer Milchviehbetriebe (Stand: Oktober 2002) in der EU dargestellt.

Im Rahmen des zuvor genannten EU-Projektes (ELPEN) hat das Institut für Betriebswirtschaft der FAL Braunschweig den direkten Zugriff zu FADN-Daten erhalten. Der Erfassungsbereich des FADN ist in den EU-Mitgliedsländern unterschiedlich (vgl. Kapitel 4.2.3). Der Umfang der Datengrundlage variiert in Abhängigkeit von der Produktionsrichtung. Da die FADN-Datensätze den Mitgliedstaaten zeitlich verzögert zur Verfügung stehen (siehe Kapitel 4.2.3), wurden die Jahre 1997 bis 1999 auf den Umfang der Datengrundlage hin überprüft. Es wurde festgestellt, dass für das Jahr 1999 ein verhältnismäßig umfassender und für diese Analyse geeigneter europäischer Datensatz im Bereich Milcherzeugung zur Verfügung steht.

$24 \quad$ ELPEN = European Livestock Policy Evaluation Network; Laufzeit von 2000 bis 2003.

25 Das Netzwerk wurde durch folgende Gebiete erweitert: Frankreich, Spanien, Österreich, Niederlande, Dänemark und Finnland. 
Karte 6.1: $\quad$ IFCN-Netzwerk typischer Milchviehbetriebe in der EU (Stand: Oktober 2002)

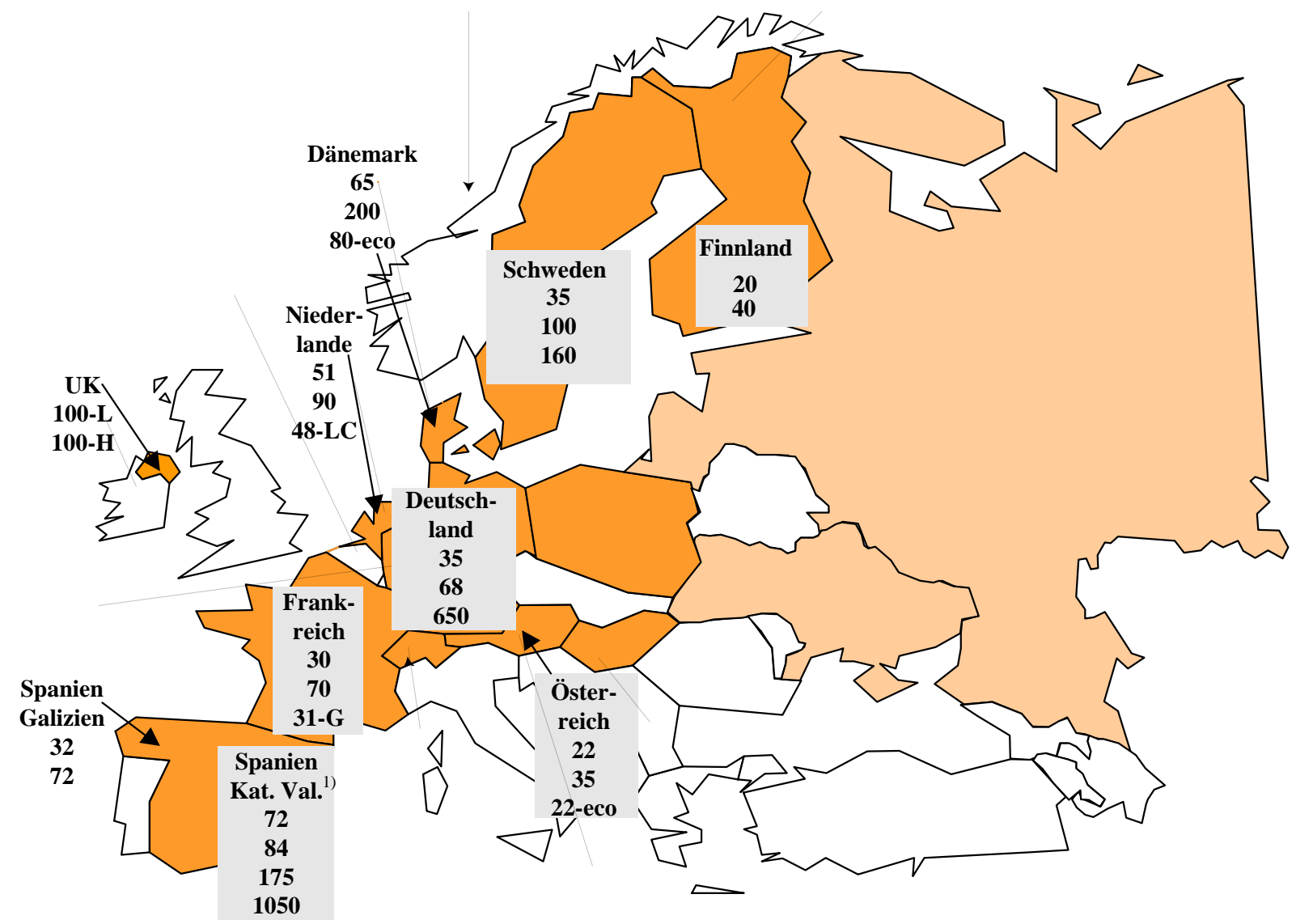

L = Lowland; H = Highland; LC = Low cost production system; eco = Ecological system; G = Grassland. Zahlen: Anzahl Milchkühe pro erhobenen Betrieb.

1) Katalunien; Valencia

Quelle: Eigene Darstellung in Anlehnung an Hemme et al. (2002).

\section{Schlussfolgerung für die Auswahl der Regionen}

Ausgehend von den zuvor erläuterten Analysen werden für den ersten Teil der empirischen Analyse folgende Untersuchungsregionen ausgewählt, die der regionalen Bedeutung der Milchproduktion Rechung tragen und ein relativ breites Betriebsgrößenspektrum widerspiegeln:

- $\quad$ Nord-, Süd- und Ostdeutschland (drei Betriebe)

- Nordspanien (zwei Betriebe)

- Ost- und Westschweden (zwei Betriebe).

Auf Basis der regionalen Betriebsstrukturentwicklung (s. Kapitel 2, Abbildung 2.1) werden in Anlehnung an das IFCN-Konzept jeweils ein durchschnittlich großer und darüber hinaus ein größerer Betrieb in die Analyse einbezogen, um Größeneffekte abbilden zu können. Die 
typischen IFCN-Betriebe sollen sowohl schrumpfende als auch wachsende Betriebsgrößenklassen $^{26}$ in den Untersuchungsregionen darstellen (siehe auch Tabellen im Anhang A6.5 bis A6.7/tabellarisch). In Deutschland werden, anders als in Schweden und Spanien, drei Betriebe ausgewählt, um die unterschiedlichen Produktionsregionen möglichst gut abzudecken. In Tabelle 6.4 sind die ausgewählten Untersuchungsbetriebe aus dem IFCNNetzwerk kurz charakterisiert.

Tabelle 6.4: $\quad$ Beschreibung der ausgewählten typischen Milchviehbetriebe

\begin{tabular}{|c|c|c|c|c|c|c|c|c|}
\hline \multicolumn{2}{|l|}{ Spezifikation } & SE-50 & SE-160 & ES-42 & ES-175 & D-35 & D-68 & D-650 \\
\hline \multicolumn{2}{|l|}{ Region } & $\begin{array}{l}\text { Schweden } \\
\text { West }\end{array}$ & $\begin{array}{l}\text { Schweden } \\
\text { Ost }\end{array}$ & Katalunien & Katalunien & $\begin{array}{l}\text { Deutschland } \\
\text { Süd }\end{array}$ & $\begin{array}{l}\text { Deutschland } \\
\text { Nord }\end{array}$ & $\begin{array}{c}\text { Deutschland } \\
\text { Ost }\end{array}$ \\
\hline \multicolumn{2}{|l|}{ Rechtsform } & Indiv.Betrieb & Ltd. & Indiv. Betrieb & $\begin{array}{l}\text { “Dairy farm } \\
\text { company” } \\
\text { Milchproduktions- } \\
\text { unternehmen }\end{array}$ & Indiv. Betrieb & Indiv. Betrieb & $\mathrm{GmbH}$ \\
\hline Anzahl Kühe & & 50 & 160 & 42 & 175 & 35 & 68 & 650 \\
\hline \multicolumn{9}{|l|}{ Betriebsbeschreibung } \\
\hline $\begin{array}{l}\text { Nutzfläche (LN) gesamt } \\
\text { Grünland (\% der }\end{array}$ & ha & 105 & 212 & 31 & 0 & 39 & 90 & 1700 \\
\hline gesamten LN) & $\%$ d. LN & 23 & 11 & 0 & - & 75 & 40 & 33 \\
\hline Lohnarbeitskräfte & AK & 0,75 & 4 & 0 & 3 & 0 & 0,2 & 34,6 \\
\hline Familienarbeitskräfte & AK & 1 & 1 & 1 & 0 & 1,5 & 2 & 0 \\
\hline Andere Produktionszwei & & & Wald & & & & Mastbullen & \\
\hline \multicolumn{9}{|l|}{ Betriebszweig Milch } \\
\hline Milchquote & $\mathrm{t}$ & 417 & 1400 & 415 & 1798 & 202 & 512 & 5033 \\
\hline Quotenkauf seit 1983 & $\%$ & $20 \%$ & $25 \%$ & $0 \%$ & $1 \%$ & $11 \%$ & $14 \%$ & $5 \%$ \\
\hline Anteil Pachtquote & $\%$ & $0 \%$ & $0 \%$ & $0 \%$ & $0 \%$ & $22 \%$ & $18 \%$ & $0 \%$ \\
\hline
\end{tabular}

Quelle: Eigene Darstellung.

\subsection{2 Ähnlichkeit der FADN-Vergleichsbetriebe und Wahl der FADN- Vergleichsgruppen}

Bei der Auswahl von FADN-Vergleichsbetrieben zum typischen IFCN-Betrieb sind vor allem die beiden Aspekte Repräsentativität der FADN-Vergleichsbetriebe und Selektionsgenauigkeit zu berücksichtigen. Für die oben kurz charakterisierten sieben IFCN-Betriebe in Deutschland, Schweden und Spanien wird die in Kapitel 6.2.1 beschriebene Methodik angewendet. Mit Hilfe der Berechnung unterschiedlicher Stichprobenzusammensetzungen wird versucht, eine dem typischen Betrieb möglichst ähnliche Gruppe von FADN-Betrieben zu ermitteln. 
Abbildung 6.3 stellt das Ähnlichkeitsmerkmal, die kumulierte euklidische Distanz, für die durch die typischen IFCN-Betriebe repräsentierten Betriebe aus der Grundgesamtheit dar. Die FADN-Betriebe (maximal 25) mit der höchsten Ähnlichkeit zum jeweiligen typischen IFCN-Betrieb werden mit ihren Hochrechnungsfaktoren multipliziert und anschließend zur Ermittlung der kumulierten euklidischen Distanz aufaddiert. Auf der x-Achse ist die Anzahl der abgebildeten Betriebe aus der Grundgesamtheit dargestellt. Die y-Achse spiegelt das Ähnlichkeitsmaß, die kumulierte euklidische Distanz, wider.

Wie Abbildung 6.3 zeigt, fallen die Ergebnisse bezüglich der Ähnlichkeit der FADNBetriebsgruppe zum entsprechenden typischen Betrieb und der Anzahl repräsentierter Betriebe aus der Grundgesamtheit recht unterschiedlich aus. Ähnlichkeitswerte bis zu 1,2 signalisieren, dass die typischen Betriebe in ihrer Region relativ gut durch die FADNVergleichsbetriebe abgebildet werden. Dazu zählen der 35-Kuhbetrieb in Bayern/Süddeutschland, der 68-Kuhbetrieb in Niedersachsen/Norddeutschland, der 42-Kuhbetrieb in Katalonien/Nordspanien und der 50-Kuhbetrieb in Westschweden.

Bei einem Ähnlichkeitswert von 1,2 werden beispielsweise in Niedersachsen durch den IFCN-Betrieb mit 68 Kühen (D-68 SHNI) ca. 400 Betriebe aus der Grundgesamtheit repräsentiert. Die um schleswig-holsteinische Betriebe erweiterte Stichprobe signalisiert, dass der typische IFCN-Betrieb bei einem Ähnlichkeitsmaß von 1,0 ca. 700 Betriebe aus der Grundgesamtheit repräsentiert, das entspricht ca. $5 \%$ der Milchviehbetriebe in Niedersachsen und Schleswig-Holstein. Im Vergleich dazu sind zum IFCN 650-Kuhbetrieb (D650 ST) in Sachsen-Anhalt sowohl in Sachsen-Anhalt, als auch in den neuen Bundesländern keine ähnlichen FADN-Betriebe (euklidische Distanz >1,2.) zu finden. Die Betriebe aus der FADN-Stichprobe in Bayern zeigen hingegen wieder eine hohe Ähnlichkeit zum typischen IFCN-Betrieb (euklidische Distanz <1,0). Bei einem Ähnlichkeitswert von 0,9 werden ca. 1.500 Betriebe aus Bayern durch den IFCN-Betrieb repräsentiert.

Im Hinblick auf die spanischen IFCN-Betriebe wird in Abbildung 6.3 deutlich, dass der katalonische 42-Kuhbetrieb (ES-42 CA) einige Betriebe mit einer hohen Ähnlichkeit repräsentiert. Ab einer bestimmten Anzahl Betriebe aus der FADN-Stichprobe reduziert sich die Ähnlichkeit jedoch erheblich. Werden neben Katalonien alle Regionen Nordspaniens einbezogen (ES-42 ES-Nord), weisen die 25 ähnlichsten FADN-Betriebe eine euklidische Distanz von 1,1-1,4 auf. Der katalonische IFCN 175-Kuhbetrieb (ES 175 CA) spiegelt deutlich schlechter die regionalen Strukturen wider (euklidische Distanz $>3$ ).

Hinsichtlich der schwedischen IFCN-Betriebe ist festzustellen, dass der westschwedische 50-Kuhbetrieb bei einem Ähnlichkeitskriterium von 1,2 ca. 800 Betriebe der Region repräsentiert. Für den ostschwedischen 160-Kuhbetrieb sind keine FADN-Betriebe mit Ähnlichkeitswerten unter 1,2 zu finden. 
Abbildung 6.3: Kumulierte euklidische Distanz in Abhängigkeit von der Anzahl abgebildeter Betriebe aus der Grundgesamtheit
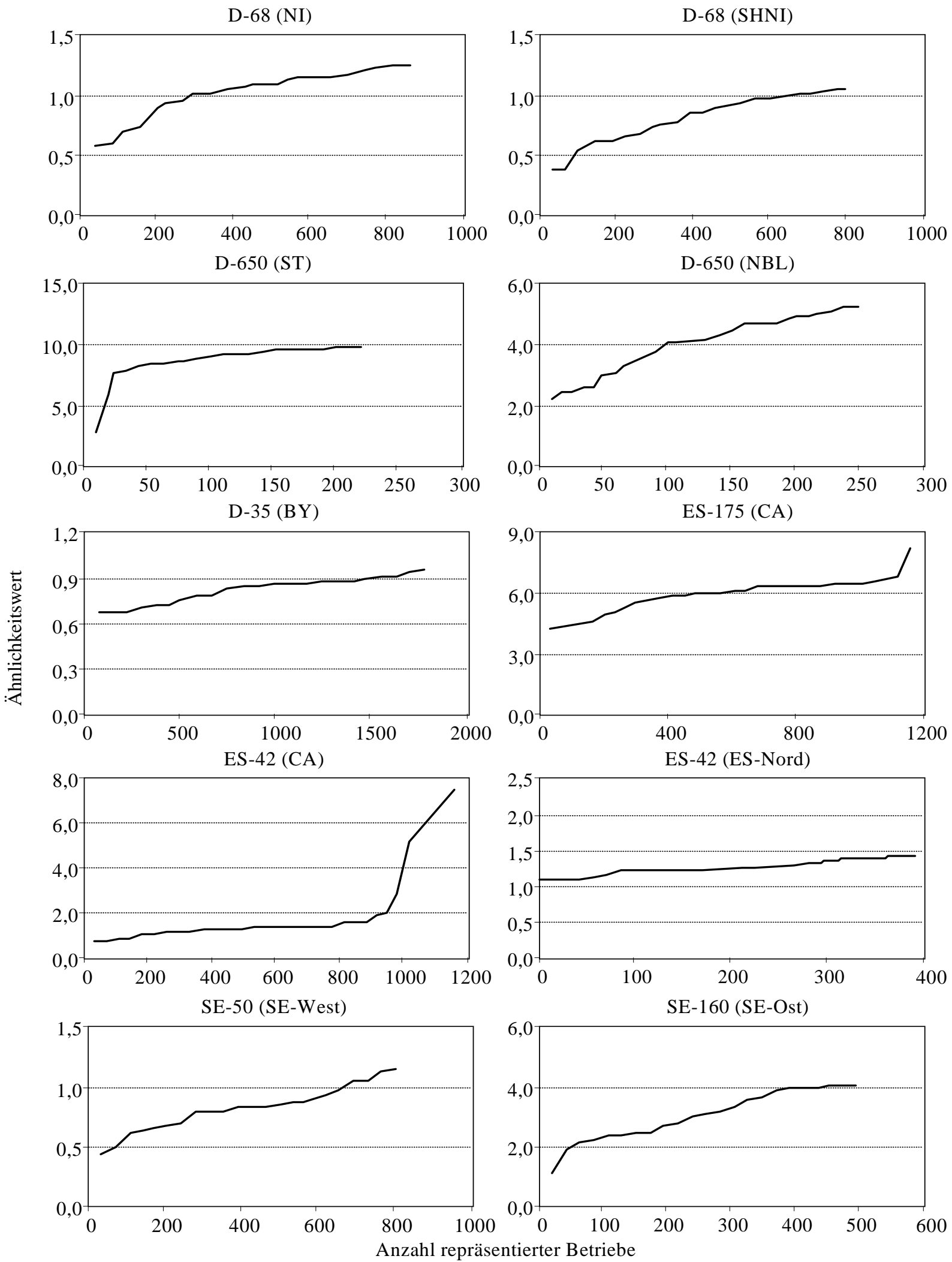

Quelle: Eigene Berechnung und Darstellung. 
Eine Ursache dafür, dass für den 650-Kuhbetrieb in Sachsen-Anhalt, den ostschwedischen 160-Kuhbetrieb und den katalonischen 175-Kuhbetrieb kaum ähnliche Betriebe im FADN-Datensatz zu finden sind, kann auch an der hier gewählten Methodik liegen, die ausschließlich die Betriebe einbezieht, die auf Milchproduktion spezialisiert sind. Bei den Großbetrieben in Ostdeutschland beispielsweise handelt es sich häufig um Gemischtbetriebe, die mehrere spezialisierte Einheiten (Milcherzeugung, Ackerbau etc.) unter einem Unternehmensdach vereinen und deswegen statistisch als Gemischtbetriebe eingestuft werden. Darüber hinaus ist zu beachten, dass die großen Betriebe entsprechend der IFCN-Methodik als Wachstumsbetriebe (möglicherweise zukünftig als typisch einzustufende Betriebe) gelten. Von daher verwundert es auch nicht, dass es schwierig ist, für diese Betriebe Vergleichsbetriebe zu finden.

Entsprechend der zuvor beschriebenen Vorgehensweise stellt Abbildung 6.4 die kumulierte euklidische Distanz für die durch die typischen IFCN-Betriebe repräsentierte Milchmenge aus der Grundgesamtheit dar. Der Anteil der abgebildeten Milchmenge variiert wie auch schon zuvor für die Anzahl der Betriebe gezeigt - in Abhängigkeit vom Hochrechnungsfaktor der FADN-Vergleichsbetriebe deutlich zwischen den verschiedenen Ländern und Betrieben.

Bei einem Ähnlichkeitsmaß von 1,2 repräsentiert der niedersächsische 68-Kuhbetrieb (D68 NI) beispielsweise ca. 200.000 Tonnen der in Niedersachsen erzeugten Milch, das entspricht bei einer Milchleistung von ca. 8 Tonnen je Kuh und Jahr ca. 300 bis 400 Betrieben. Die um schleswig-holsteinische Betriebe erweiterte Stichprobe (D-68 SHNI) signalisiert, dass der typische IFCN-Betrieb bei einem Ähnlichkeitsmaß von 1,2 ca. 300.000 Tonnen erzeugte Milch aus der Grundgesamtheit repräsentiert. Wie bereits oben erwähnt, wird in Abbildung 6.4 ebenso deutlich, dass der bayerische 35-Kuhbetrieb (D-35 BY) sehr gut die Strukturen in Bayern repräsentiert. Für den 650-Kuhbetrieb in Sachsen-Anhalt sind nur sehr wenig strukturell vergleichbare Betriebe im FADN-Datensatz zu finden (siehe oben). Dies spiegelt sich in der hohen euklidischen Distanz wider. Auf Basis der Daten des statistischen Bundesamtes beträgt der Anteil der Betriebe mit mehr als 500 Kühen an der Milcherzeugung in Sachsen-Anhalt ca. $18 \%$ (Statistisches Bundesamt, 2000). Beim spanischen 42-Kuhbetrieb (ES-42 CA) wird in Abbildung 6.4 wiederum deutlich, dass nur wenige ähnliche Betriebe aus dem FADN existieren. Der schwedische 50-Kuhbetrieb (SE50 SE-West) spiegelt bei einem Ähnlichkeitskriterium von 1,2 ca. 250.000-300.000 Tonnen der in Westschweden erzeugten Milch wider, dies entspricht ca. 800 Betrieben (vgl. Abbildung 6.3). 
Abbildung 6.4: Kumulierte euklidische Distanz in Abhängigkeit von der abgebildeten Milchmenge aus der Grundgesamtheit (in Tonnen)
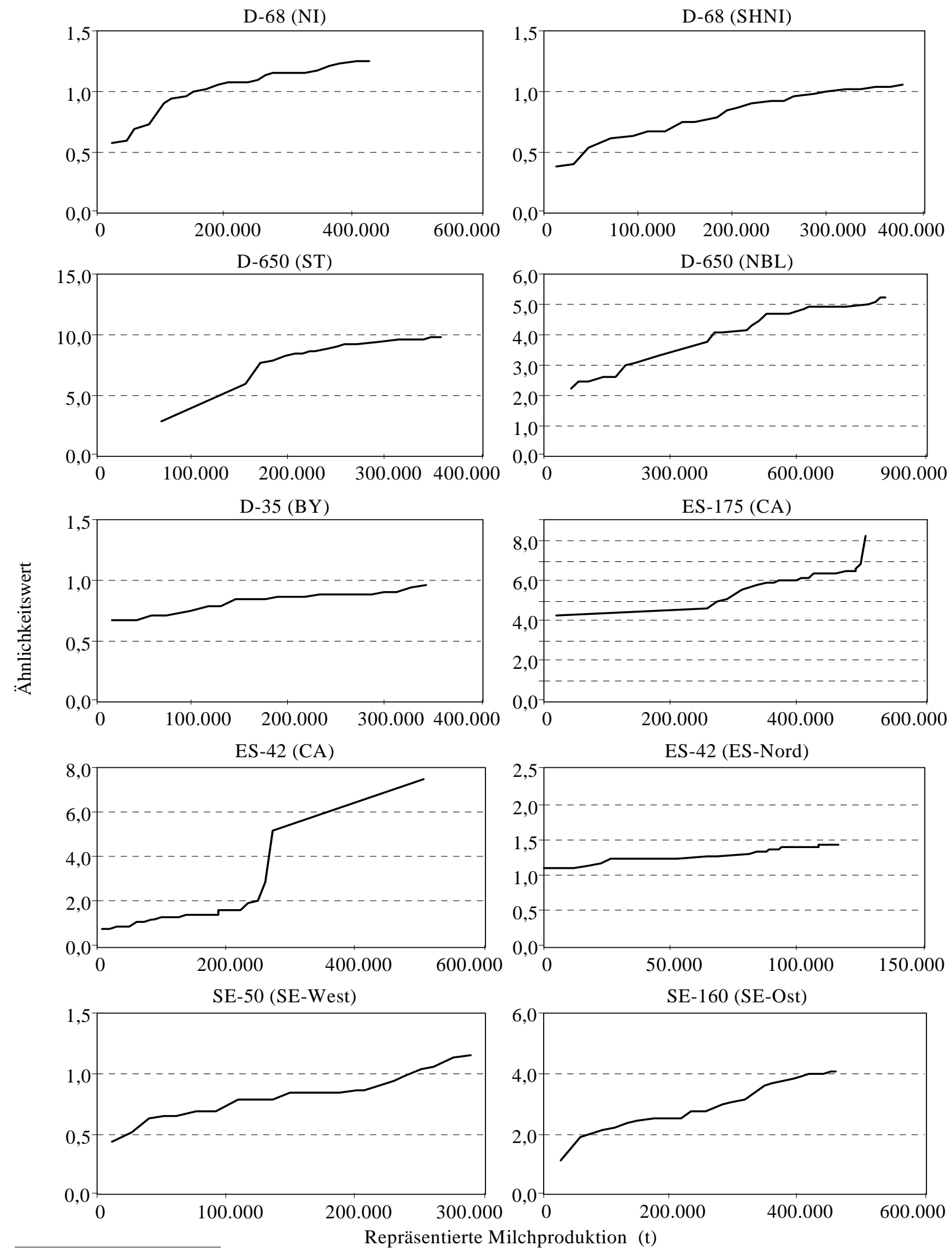

Quelle: Eigene Berechnungen und Darstellung. 
In der Tabelle 6.5 wird den IFCN-Betrieben die Anzahl der in dieser Untersuchung betrachteten FADN-Betriebe und der von den FADN-Betrieben abgebildete Ausschnitt aus der Grundgesamtheit (Anzahl Betriebe und Prozentanteil der Milchviehbetriebe pro Land) gegenübergestellt. Es sind nur die Betriebe in der Tabelle 6.5 aufgeführt, die den Berechnungen zufolge relativ häufig in den Regionen anzutreffen sind. Da für den 650Kuhbetrieb in Sachsen-Anhalt, den 175-Kuhbetrieb in Katalonien und den 160-Kubetrieb in Ostschweden nur sehr wenige Betriebe vergleichbarer Größenklasse zu finden sind, würde die tabellarische Darstellung der Werte nur zu Missinterpretationen führen und ist auch aus Datenschutzgründen nicht möglich.

Tabelle 6.5: Prozentualer Anteil der abgebildeten Milchviehbetriebe in Deutschland, Schweden und Spanien in Abhängigkeit von der Ähnlichkeitszahl

\begin{tabular}{|c|c|c|c|c|c|c|c|}
\hline & & $\begin{array}{c}\text { NI } \\
\text { D-68 }\end{array}$ & $\begin{array}{c}\text { NI + SH } \\
\text { D-68 }\end{array}$ & $\begin{array}{c}\text { CA } \\
\text { ES-42 }\end{array}$ & $\begin{array}{c}\text { CA + CN + } \\
\text { AS }+ \text { PV } \\
\text { ES-42 }\end{array}$ & $\begin{array}{c}\text { BY } \\
\text { D-35 }\end{array}$ & $\begin{array}{c}\text { SE } \\
\text { SE-50 }\end{array}$ \\
\hline Ähnlichkeitskennzahl & & 1,03 & 0,82 & 1,84 & 1,29 & 0,82 & 0,83 \\
\hline FADN & Anzahl Betriebe & 25 & 25 & 25 & 25 & 25 & 25 \\
\hline Grundgesamtheit & Anzahl Betriebe & 864 & 803 & 1.163 & 394 & 1.784 & 807 \\
\hline \multicolumn{8}{|c|}{ \%-Anteil der repräsentierten Milchviehbetriebe } \\
\hline pro Region (1999) ${ }^{1)}$ & $\%$ & 3,8 & 2,7 &.. &.. & 2,6 & .. \\
\hline pro Land (1999) ${ }^{2)}$ & $\%$ & 0,6 & 0,5 & 1,7 & 0,6 & 1,2 & 5,7 \\
\hline
\end{tabular}

1) Statistisches Bundesamt, Fachserie 2, Reihe 4, Viehbestand und tierische Erzeugung, verschiedene Jahre; EUROSTAT Databank, New Cronos 2002, Agrarstatistik; EUROSTAT, Statistisches Jahrbuch der Landwirtschaft, verschiedene Jahre. Erläuterung: .. = Zahlwert nicht vorhanden.

2) Statistisches Bundesamt, Fachserie 2, Reihe 4, Viehbestand und tierische Erzeugung, verschiedene Jahre; EUROSTAT Databank, New Cronos 2002, Agrarstatistik; EUROSTAT, Statistisches Jahrbuch der Landwirtschaft, verschiedene Jahre.

Quelle: INLB-EU_GD-AGRI/G3. Eigene Berechnung und Darstellung.

Wie Tabelle 6.5 zeigt, hat die Ähnlichkeitskennzahl in der nur die niedersächsischen Betriebe berücksichtigenden Gruppe höhere Werte als in der um Schleswig-Holstein erweiterten Gruppe. Wie schon der Abbildung 6.3 entnommen werden kann, führt demzufolge die erweiterte FADN-Betriebsgruppe bestehend aus schleswig-holsteinischen und niedersächsischen Betrieben zu besseren Ähnlichkeitswerten. Durch die größere Anzahl von Betrieben im Auswahlpool hat sich die Wahrscheinlichkeit erhöht, Betriebe ähnlicher Betriebsstruktur zu finden. Dies führt zu einer Verringerung der euklidischen Distanz. Dieses Beispiel zeigt, dass die niedersächsischen FADN-Betriebe den schleswig-holsteinischen FADN-Betrieben ähnlich sind. Dies bedeutet wiederum, dass in diesem Beispielsfall möglicherweise Informationen und Analyseergebnisse von einem IFCN-Betrieb auf FADNBetriebe aus unterschiedlichen Regionen übertragen werden können. 
Ähnliches lässt sich für die Vergleichsbetriebe zum spanischen 42-Kuhbetrieb feststellen. Allerdings zeigt sich, dass die ersten Betriebe aus der katalonischen Betriebsgruppe bezogen auf die Ähnlichkeit deutlich besser liegen als die horizontal verlaufenden Distanzwerte der nordspanischen Vergleichsbetriebe (vgl. Abbildung 6.3). In Bezug auf die Ähnlichkeitskennzahl (siehe Tabelle 6.5) zeigt die katalonische Betriebsstichprobe jedoch einen schlechteren Wert als die nordspanischen Vergleichsbetriebe. Die ersten 900 Betriebe zeigen eine sehr hohe Ähnlichkeit zum Vergleichsbetrieb. Die übrigen Betriebe der katalonischen Betriebsgruppe weichen deutlich ab. Das bedingt, dass sich die durchschnittliche Ähnlichkeitskennzahl der katalonischen Vergleichsbetriebe verschlechtert. Die Gruppengröße sollte daher - nicht wie in dieser Arbeit vorgenommen - von vornherein auf eine bestimmte Anzahl Betriebe festgelegt werden. Zu prüfen wäre, ob es nicht sinnvoller ist, als Abschneidegrenze eine bestimmte Ähnlichkeitskennzahl zu wählen.

Wie der Tabelle 6.5 darüber hinaus entnommen werden kann, ist der von der jeweiligen FADN-Vergleichsgruppe abgebildete Ausschnitt aus der Grundgesamtheit pro Land noch relativ gering (0,5 bis 5,7\%). Dies liegt unter anderem auch an der festgelegten Zahl von maximal 25 Untersuchungsbetrieben mit der geringsten euklidischen Distanz zum IFCNBetrieb. Der prozentuale Anteil der abgebildeten Betriebe in den Bundesländern Niedersachsen, Schleswig-Holstein und Bayern variiert zwischen 2,6 und 3,8 \%.

Tabelle 6.6 zeigt den prozentualen Anteil der Milchproduktion pro Land, der von den IFCN-Betrieben diesen Berechnungen zufolge abgebildet wird. Der prozentuale Anteil der gesamten Milchproduktion variiert zwischen 1,2 (D-35, Bayern) und 8,8 \% (SE-50, Westschweden). Unterschiedlich stellt sich wieder der abgebildete Ausschnitt der Grundgesamtheit in den untersuchten Regionen dar: er variiert zwischen 4,6 \% (D-35, Bayern) und $79 \%$ (ES-42, Cataluna).

Tabelle 6.6: Prozentualer Anteil der abgebildeten Milchproduktion in Deutschland, Schweden und Spanien in Abhängigkeit von der Ähnlichkeitszahl

\begin{tabular}{|c|c|c|c|c|c|c|}
\hline Anzahl Betriebe & $\begin{array}{c}\text { NI } \\
\text { D-68 }\end{array}$ & $\begin{array}{c}\mathrm{NI}+\mathrm{SH} \\
\mathrm{D}-68\end{array}$ & $\begin{array}{c}\text { CA } \\
\text { ES-42 }\end{array}$ & $\begin{array}{c}\text { CA + CN + } \\
\text { AS + PV } \\
\text { ES-42 }\end{array}$ & $\begin{array}{c}\text { BY } \\
\text { D-35 }\end{array}$ & $\begin{array}{c}\text { SE } \\
\text { West } \\
\text { SE-50 }\end{array}$ \\
\hline Ähnlichkeitskennzahl & 1,03 & 0,82 & 1,84 & 1,29 & 0,82 & 0,83 \\
\hline FADN Anzahl Betriebe & 25 & 25 & 25 & 25 & 25 & 25 \\
\hline $\begin{array}{l}\text { Milchmenge (t) der repräsentierten } \\
\text { Grundgesamtheit }\end{array}$ & 426.767 & 379.273 & 508.544 & 117.141 & 344.421 & 291.515 \\
\hline \multicolumn{7}{|l|}{ \%-Anteil der repräsentierten Milchproduktion } \\
\hline pro Region (1999) ${ }^{1)} \%$ & 8,0 & 4,9 & 79,0 & 5,8 & 4,6 & 34,5 \\
\hline pro Land (1999) ${ }^{1)}$ & 1,5 & 1,3 & 8,3 & 1,9 & 1,2 & 8,7 \\
\hline
\end{tabular}

1) EUROSTAT Databank, New Cronos 2002, Agrarstatistik.

Quelle: INLB-EU_GD-AGRI/G3. Eigene Berechnung und Darstellung. 
Grundsätzlich gilt, dass je mehr FADN-Betriebe in der Vergleichsgruppe zugelassen werden, desto größer zwar der entsprechende Ausschnitt aus der Grundgesamtheit ist, aber desto geringer die Ähnlichkeit des Durchschnitts der FADN-Vergleichsbetriebe zum IFCN-Betrieb. Bei einer ungünstigeren Ähnlichkeitszahl würde sich die Anzahl der repräsentierten Betriebe erhöhen, die Gruppe würde jedoch inhomogener. Dem schließt sich die Frage an: Wie groß ist der Fehler, wenn eine geringere Ähnlichkeit in Kauf genommen wird zu Gunsten einer erhöhten Repräsentativität von beispielsweise etwa 8 \% oder sogar $20 \%$ der Grundgesamtheit?

Wie Tabelle 6.7 zeigt, erhöht sich die Repräsentativität des typischen niedersächsischen Betriebs bezogen auf die Grundgesamtheit auf 8 bzw. $20 \%$, wenn anstelle von maximal 25 Betrieben 60 bzw. 150 FADN-Betriebe mit der höchsten Ähnlichkeit zum typischen IFCN-68-Kuhbetrieb zugeordnet werden. Das euklidische Distanzmaß erhöht sich dabei um 0,3 bzw. 0,6 Prozentpunkte. Um den Fehler noch besser einschätzen zu können, wird die Standardabweichung einiger Strukturvariablen (Milchquote, Milchkühe, landw. Nutzfläche, Grünland, Arbeitskräfte) bei einer Repräsentativität von 8 und 20 \% im Vergleich zur Referenz von 3 \% berechnet. Tabelle 6.8 und Tabelle 6.9 zeigen, dass die Standardabweichung bei steigender Repräsentativität erwartungsgemäß ansteigt. Die Abweichungen vom Mittelwert bei einer Repräsentativität von $3 \%$ sind beim 68-Kuhbetrieb mit maximal $14 \%$ noch relativ gering. Die Mittelwerte zeigen - mit Ausnahme des bayerischen 35Kuhbetriebes, bezogen auf die Variable Grünland - in den verschiedenen Gruppen ähnliche Größenordnungen.

Tabelle 6.7: $\quad$ Prozentualer Anteil der abgebildeten Milchviehbetriebe aus der Grundgesamtheit

\begin{tabular}{|c|c|c|c|c|c|c|}
\hline \multirow{2}{*}{$\begin{array}{l}\text { Anzahl Betriebe } \\
\text { IFCN }\end{array}$} & \multicolumn{3}{|c|}{$\begin{array}{c}\mathrm{NI}+\mathrm{SH} \\
\mathrm{D}-68\end{array}$} & \multicolumn{3}{|c|}{$\begin{array}{c}\text { BY } \\
\text { D-35 }\end{array}$} \\
\hline & 1 & 1 & 1 & 1 & 1 & 1 \\
\hline FADN & 25 & 60 & 150 & 25 & 48 & 165 \\
\hline Grundgesamtheit & 864 & 2.087 & 5.431 & 1.784 & 3.591 & 12.242 \\
\hline \multicolumn{7}{|c|}{$\%$ Anteil der Milchviehbetriebe } \\
\hline pro Region (1999) ${ }^{1)}$ & 3,8 & 7,6 & 20,0 & 2,6 & 7,6 & 20,0 \\
\hline pro Land (1999) ${ }^{2)}$ & 0,6 & 1,1 & 3,0 & 1,2 & 2,0 & 7,0 \\
\hline Ähnlichkeitskennzahl & 0,8 & 1,1 & 1,4 & 0,8 & 1,0 & 1,4 \\
\hline
\end{tabular}

1) Statistisches Bundesamt, Fachserie 2, Reihe 4, Viehbest and und tierische Erzeugung, verschiedene Jahre; EUROSTAT Databank, New Cronos 2002, Agrarstatistik; EUROSTAT, Statistisches Jahrbuch der Landwirtschaft, verschiedene Jahre. Erläuterung: .. = Zahlwert nicht vorhanden.

2) Statistisches Bundesamt, Fachserie 2, Reihe 4, Viehbestand und tierische Erzeugung, verschiedene Jahre; EUROSTAT Databank, New Cronos 2002, Agrarstatistik; EUROSTAT, Statistisches Jahrbuch der Landwirtschaft, verschiedene Jahre. Quelle: INLB-EU_GD-AGRI/G3. Eigene Berechnung und Darstellung. 
Tabelle 6.8: Mittelwert und Standardabweichung bei unterschiedlicher Repräsentativität der verschiedenen FADN-Vergleichsgruppen zum IFCN-68-Kuhbetrieb in Niedersachsen

\begin{tabular}{|c|c|c|c|c|c|c|}
\hline \% Grundgesamtheit & $\begin{array}{c}\text { Anzahl } \\
\text { FADN-Bet riebe }\end{array}$ & $\begin{array}{c}\text { Milchquote } \\
1.000 \mathrm{t}\end{array}$ & $\begin{array}{c}\text { Milchkühe } \\
\text { Stückzahl }\end{array}$ & $\begin{array}{l}\text { LF } \\
\text { ha }\end{array}$ & $\begin{array}{l}\text { GL } \\
\text { ha }\end{array}$ & $\begin{array}{c}\text { AK } \\
\text { Einheiten }\end{array}$ \\
\hline \multicolumn{7}{|l|}{ Mittelwert } \\
\hline bei 3\% & 882 & 474 & 66 & 84 & 40 & 2,2 \\
\hline bei 8 \% & 2087 & 465 & 64 & 79 & 40 & 2,0 \\
\hline bei $20 \%$ & 5407 & 431 & 61 & 73 & 44 & 1,9 \\
\hline \multicolumn{7}{|l|}{ Standardabweichung } \\
\hline bei 3\% & 882 & 75 & 9 & 12 & 11 & 0,3 \\
\hline bei $8 \%$ & 2087 & 83 & 10 & 15 & 13 & 0,4 \\
\hline bei $20 \%$ & 5407 & 105 & 13 & 17 & 15 & 0,5 \\
\hline
\end{tabular}

Quelle: INLB-EU-GD-AGRI/G3; eigene Berechnungen.

Tabelle 6.9: Mittelwert und Standardabweichung bei unterschiedlicher Repräsentativität der verschiedenen FADN-Vergleichsgruppen zum IFCN-35-Kuhbetrieb in Bayern

\begin{tabular}{|c|c|c|c|c|c|c|}
\hline$\%$ Grundgesam th eit & $\begin{array}{c}\text { Anzahl } \\
\text { FAD N-Betriebe }\end{array}$ & $\begin{array}{l}\text { Mildh quote } \\
1.000 \mathrm{t}\end{array}$ & $\begin{array}{c}\text { Milchkü he } \\
\text { Stückzahl }\end{array}$ & $\begin{array}{l}\text { LF } \\
\text { ha }\end{array}$ & $\begin{array}{l}\text { GL } \\
\text { ha }\end{array}$ & $\begin{array}{c}\text { AK } \\
\text { Einheiten }\end{array}$ \\
\hline \multicolumn{7}{|l|}{ Mittelwert } \\
\hline bei 3\% & 1867 & 193 & 32 & 34 & 30 & 1,5 \\
\hline bei 8 \% & 3591 & 194 & 33 & 34 & 23 & 1,5 \\
\hline bei $20 \%$ & 12242 & 185 & 32 & 33 & 44 & 1,5 \\
\hline \multicolumn{7}{|l|}{ Stand ardab weichung } \\
\hline bei 3\% & 1867 & 29 & 4 & 6 & 4 & 0,1 \\
\hline bei 8 \% & 3591 & 32 & 4 & 6 & 6 & 0,2 \\
\hline bei $20 \%$ & 12242 & 46 & 6 & 10 & 7 & 0,2 \\
\hline
\end{tabular}

Quelle: INLB-EU-GD-AGRI/G3; eigene Berehnungen.

Die gewählte Vorgehensweise zur Auswahl von Vergleichsbetrieben ist beispielsweise erweiterbar, indem nur diejenigen Betriebe einbezogen werden, die ein bestimmtes Distanzmaß (z. B. 2,0) nicht überschreiten. Dies würde im Fall der katalonischen Vergleichsgruppe zwar zu einer geringeren Anzahl Betriebe, aber zu einer größeren Homogenität der Vergleichsbetriebe führen.

Wie die zuvor erläuterten Analysen zeigen, lässt sich mit Hilfe des euklidischen Distanzmaßes gut feststellen, ob zu den typischen IFCN-Betrieben ähnliche Betriebe in der 
FADN-Stichprobe vorliegen und wie viele Betriebe aus der Grundgesamtheit von diesen repräsentiert werden. Hierbei ist jedoch zu beachten, dass die Repräsentanz je nach gewählter Ähnlichkeitszahl stark variieren kann und es keine objektive Regel gibt, welches Distanzmaß gerade noch akzeptabel ist.

Von daher stellt sich die Frage, ob der FADN-Datensatz nicht schon besser bei der Auswahl der typischen Betriebe genutzt werden sollte, als nachher festzustellen, wie repräsentativ die mit Hilfe von Beratern festgelegten typischen Betriebe tatsächlich sind.

Deshalb wird in Kapitel 6.5 ein Verfahren vorgestellt, wie die Auswahl typischer IFCNBetriebe auf Basis statistischer Daten (FADN-Daten) erfolgen kann. 


\subsubsection{Bewertung der Einordnung von IFCN-Betrieben mittels FADN}

Im Folgenden werden die Ergebnisse aus der Einordnung der IFCN-Betriebe in die kumulierte Verteilung der FADN-Betriebe vorgestellt. Um herauszufinden, wie typisch die IFCN-Betriebe vor dem Hintergrund der Verteilung in der Grundgesamtheit wirklich sind, wird der IFCN-Betrieb bezüglich seiner Rentabilität eingeordnet. Dies soll eine differenziertere Betrachtung der Simulationsergebnisse typischer Betriebe ermöglichen.

Die Einordnung der Datensätze FADN und IFCN (zweiter Schritt beim Verknüpfungsansatz I) wird an zwei Gruppen von FADN-Betrieben demonstriert, die eine sehr hohe Ähnlichkeit zum jeweiligen IFCN-Betrieb aufweisen (siehe Kapitel 6.2.1). Es handelt sich um die FADN-Betriebe, die a) dem IFCN 68-Kuhbetrieb in Norddeutschland und b) dem IFCN 35-Kuhbetrieb in Süddeutschland/Bayern zuzuordnen sind.

In Tabelle 6.10 werden kurz einzelne gewinnrelevante Faktoren der FADN- und IFCNBetriebe wie Strukturmerkmale, Erträge, Kostenbereiche und Preise gegenübergestellt und Unterschiede aufgezeigt. Anschließend werden die IFCN-Betriebe in die Verteilung der FADN-Betriebe anhand des Produktionskostenindikators eingeordnet. Es wird der Frage nachgegangen, für wie viele Betriebe aus der Grundgesamtheit Rückschlüsse aus den IFCN-Analysen zum MTR gezogen werden können.

In Tabelle 6.10 ist zu erkennen, dass die Herdengröße bei den berücksichtigten FADNund IFCN-Betrieben in etwa vergleichbar ist. Die Milchleistung liegt in den IFCNBetrieben tendenziell höher. Eine Ausnahme ist der 35-Kuhbetrieb in Süddeutschland. Während der typische IFCN-Betrieb eine Zweinutzungsrasse hält, ist die FADNBetriebsgruppe aus unterschiedlichen Rassen zusammengesetzt. Zudem weisen die IFCNBetriebe eine leicht höhere Flächenausstattung auf als ihre entsprechenden FADNVergleichsbetriebe. Die Spezialisierung auf Milchproduktion ist den Auswertungen von HEMmE et al. (2002, S. 108-109) entsprechend bei den IFCN-Betrieben deutlich ausgeprägter als bei den berücksichtigten FADN-Betrieben.

Wie Tabelle 6.10 verdeutlicht, weisen die ausgewählten IFCN-Betriebe höhere Nebenerlöse auf als ihre FADN-Vergleichsbetriebe. Dies ist besonders ausgeprägt beim bayerischen 35-Kuhbetrieb. Das ist vor allem darauf zurückzuführen, dass seine Herde ausschließlich aus Fleckvieh besteht. Die Direktzahlungen und die Rindererlöse sind bei den berücksichtigten IFCN-Betrieben höher (insbesondere beim typischen 35-Kuhbetrieb), bedingt durch eine genauere Erfassung der Direktzahlungen und durch rassenbedingte Unterschiede bei den Rindererlösen. 
Tabelle 6.10: Vergleich zwischen zwei ausgewählten deutschen IFCN-Betrieben mit ihren FADN-Vergleichsbetrieben (Jahr 1999)

\begin{tabular}{|c|c|c|c|c|}
\hline & $\begin{array}{c}\text { FADN } \\
\text { D-66 SHNI }\end{array}$ & $\begin{array}{c}\text { IFCN } \\
\text { D-68 NI }\end{array}$ & $\begin{array}{c}\text { FADN } \\
\text { D-32 BY }\end{array}$ & $\begin{array}{c}\text { IFCN } \\
\text { D-35 BY }\end{array}$ \\
\hline Herdengröße & 66 & 68 & 32 & 35 \\
\hline Milchleistung (kg Milch(FCM) / Kuh) & 7.212 & 7.616 & 6.049 & 5.672 \\
\hline Landwirtschaftl iche Nut zfläche (Gesamt betrieb) & 84 & 90 & 34 & 39 \\
\hline Erlöse Betriebszweig Milch (\%) & 78 & 84 & 80 & 100 \\
\hline \multicolumn{5}{|l|}{ Vergleich Neben erlöse (Euro / 100 kg Milch) } \\
\hline Rindererlöse & 4,26 & 4,53 & 6,1 & 8,3 \\
\hline Direktzahlungen & 0,98 & 0,98 & 0,1 & 5,4 \\
\hline Sonstige Erlöse & 0 & 0,449 & 0 & 0,36 \\
\hline \multicolumn{5}{|l|}{ Vergleich Kosten und Erlöse (Euro / 100 kg Milch) } \\
\hline \multicolumn{5}{|l|}{ Kosten G.-u.-V.-Rechnung abzügl. Nebenerlöse } \\
\hline Milchproduktion & 20,6 & 19,82 & 19 & 16,82 \\
\hline Nutzungskosten (Arbeit, Boden und Kapital) & 10,437 & 11,94 & 20,719 & 28,264 \\
\hline Quotenkosten & & 3,38 & & 3,09 \\
\hline Milchpreis & 29,62 & 28,42 & 30,6 & 29,49 \\
\hline \multicolumn{5}{|c|}{ Vergleich Kosten noch Kostenkomponenten (Euro / 100 kg Milch) } \\
\hline Variable Kosten & 20 & 21,61 & 22 & 26,27 \\
\hline Kapitalkosten & 2,19 & 2,85 & 3 & 3,94 \\
\hline Landkosten & 4,78 & 2,18 & 4,02 & 5,28 \\
\hline Arbeitskosten & 9,28 & 11,07 & 17 & 23,72 \\
\hline Quotenkosten & & 3,38 & & 3,09 \\
\hline \multicolumn{5}{|l|}{ Vergleich Landkosten (Euro / 100 kg Milch) } \\
\hline Landpacht & 3,27 & 1,17 & 2,06 & 2,36 \\
\hline Nutzungskosten für eigenes Land & 1,51 & 1,01 & 1,96 & 2,92 \\
\hline \multicolumn{5}{|c|}{ Vergleich der Arbei tskosten in den IFCN- und FADN-Vergleichsb etrieben (Euro / 100 kg Milch) } \\
\hline Gezahl te Löhne & 1,4 & 0,6 & 0 & 0 \\
\hline Nutzungskosten Arbeit & 8,6 & 10,5 & 17 & 24 \\
\hline Vergleich Arbeitsprodu ktivität (kg / Stunde)) & 135 & 116 & 79 & 56 \\
\hline \multicolumn{5}{|l|}{ Vergleich Entlohung (kg / Stunde) } \\
\hline Durchschnittlicher gezahlter Lohn & 12,57 & 12,88 & 13,24 & 13,29 \\
\hline Entlohnung aus der Milcherzeugung & 10,52 & 5,07 & 6,21 & 2,82 \\
\hline \multicolumn{5}{|c|}{ Vergleich Kostenaufteilung pagatorische und kal kulat ori sche Kosten (Euro/100 kg Milch) } \\
\hline Pagatorische Kosten & 22 & 21 & 17 & 24 \\
\hline Abschreibung & 4 & 4 & 8 & 7 \\
\hline Nutzungskosten & 10 & 12 & 21 & 28 \\
\hline
\end{tabular}

Quelle: Eigene Dar stellung. 
Ein Vergleich der Milchproduktionskosten hat ergeben, dass die Kosten aus der Gewinnund-Verlust-Rechnung bei beiden Datensätzen ein ähnliches Niveau haben. Unterschiedlich sind hingegen die geschätzten kalkulatorischen Kosten für familieneigene Produktionsfaktoren, die bei den IFCN-Betrieben in der Regel höher liegen als im FADNDurchschnitt, weil sie von den Experten häufig höher eingeschätzt werden, als aus den FADN-Aufzeichnungen hervorgeht. Beim Vergleich der Faktorkosten ist festzustellen, dass die Kapitalkosten bei beiden Datensätzen gut vergleichbar sind. Die höheren Arbeitskosten bei den IFCN-Betrieben werden hauptsächlich durch einen höher veranschlagten Arbeitszeitbedarf verursacht. Eine Vermutung ist, dass diese Differenz größtenteils auf Probleme bei der Erfassung der FADN-Arbeitskosten zurückzuführen ist. Letztlich muss die Frage, welche Arbeitsentlohnung die realistischere ist, offen bleiben. Zum einen ist nicht auszuschließen, dass die IFCN-Betriebsleiter ihren Arbeitsaufwand überschätzt haben. Zum anderen können die für die FADN-Betriebe ermittelten Arbeitsstunden durch den gewählten Allokationsmechanismus verzerrt und möglicherweise unterschätzt sein. Aussagen über Quotenkosten (Quotenpacht und Quotenkauf) waren im FADN-Datensatz bei den ausgewählten Betrieben nicht vorhanden. Daher wurden die Quotenkosten bei der Einordnung der typischen Betriebe in die Verteilung der FADN-Betriebe nicht berücksichtigt.

\section{Einordnung der IFCN-Betriebe in die FADN-Verteilung}

Zur Einordnung der IFCN-Betriebe in die kumulierte Verteilung der FADN-Betriebe werden - wie in Kapitel 6.3 beschrieben - als Indikatoren die Vollkosten und die Kosten aus der Gewinn-und-Verlust-Rechnung für den Betriebszweig Milch ausgewählt. ${ }^{27}$ Die Schwierigkeiten beim Vergleich der Nutzungskosten sind bei der Interpretation der Ergebnisse (bezogen auf den Vollkostenindikator) zu beachten.

Wie Abbildung 6.5 zeigt, ist der typische 68-Kuhbetrieb gut vergleichbar mit der entsprechenden FADN-Betriebsgruppe und stellt einen durchschnittlich leistungsstarken 60-bis 70-Kuhbetrieb in Norddeutschland (Niedersachsen/Schleswig-Holstein) dar. Wie bereits oben erwähnt, weist der IFCN 68-Kuhbetrieb, bezogen auf die Kosten aus der Gewinnund-Verlust-Rechnung, etwas niedrigere Kosten auf als der durchschnittliche 60- bis 70Kuhbetrieb Norddeutschlands. Auf der Ebene der Vollkosten liegt der IFCN 68Kuhbetrieb etwas höher als der Durchschnitt des FADN-Clusters, vor allem bedingt durch die höheren Nutzungskosten (siehe oben). 
Abbildung 6.5: $\quad$ Einordnung der IFCN-Betriebe in die FADN-Verteilung auf Basis der Produktionskosten - Betrieb D-68 (SH-NI)

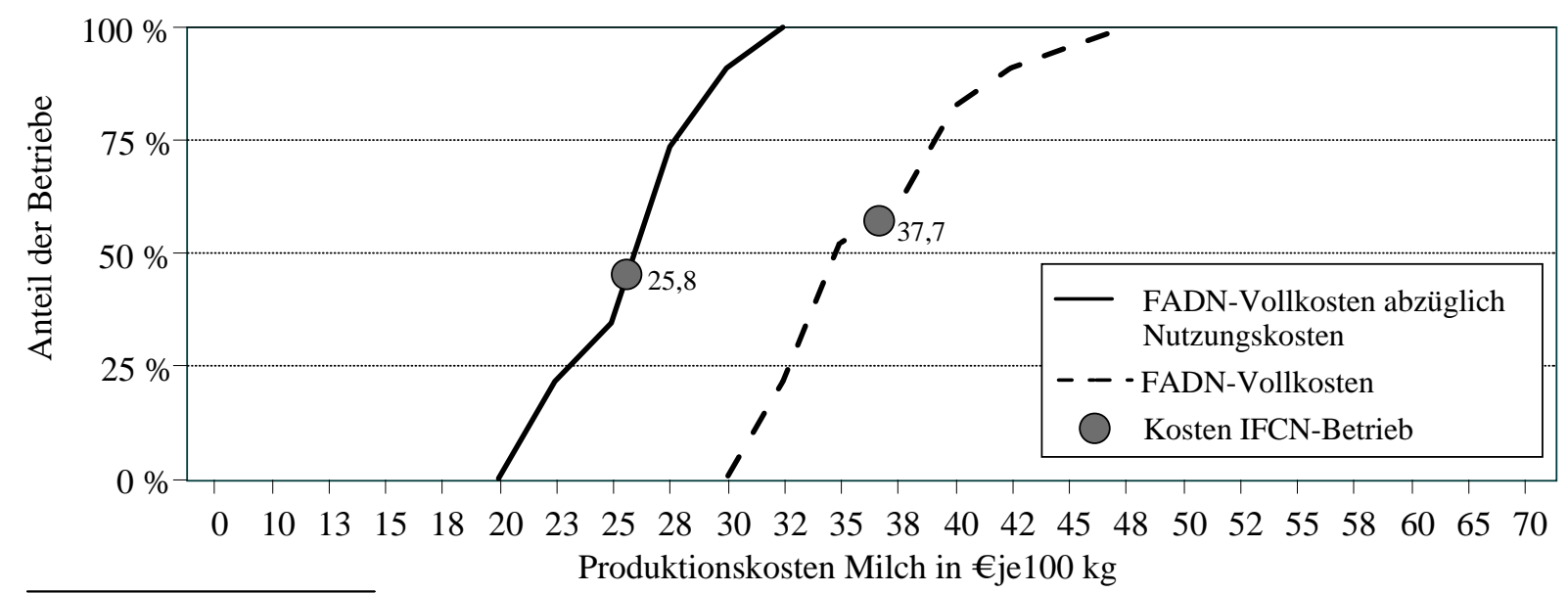

Quelle: Eigene Berechnung und Darstellung; Datenbasis: Europäisches Testbetriebsnetz (FADN, 1999/2000).

Der typische bayerische 35-Kuhbetrieb weist bedeutend höhere Kosten auf als die zu vergleichende FADN-Betriebsgruppe und stellt das obere Viertel auf der Verteilungskurve dar (siehe Abbildung 6.6). Dies trifft für beide Kostenindikatoren zu, wobei der Vollkostenindikator an der obersten Grenze liegt und nur ca. $10 \%$ der Betriebe mit den höchsten Produktionskosten widerspiegelt. Eine Vermutung ist, dass es sich bei dem IFCN-Betrieb, wie oben erwähnt, um einen Betrieb mit Fleckvieh handelt, bei dem in der Regel höhere Produktionskosten anfallen als bei 35-Kuhbetrieben milchbetonter Rassen. Die Milchleistung liegt in dem Fleckvieh-Betrieb niedriger als in der FADN-Betriebsgruppe (siehe oben), die möglicherweise eine inhomogenere Gruppe bestehend aus 25 Betrieben mit unterschiedlichen Rassen widerspiegelt. Eine Vorselektion nach der Rasse würde die Unterschiede möglicherweise verringern. Diese Variable ist im FADN-Datensatz allerdings nicht vorhanden. Da jedoch die meisten Betriebe in der Region mittlerweile Fleckvieh haben, kann es aber auch sein, dass bei der Bildung des typischen IFCN-Betriebs sich das Panel auf einen nicht-typischen Wert geeinigt hat. Die Spannbreite der Produktionskosten ist bei den FADN-Vergleichbetrieben zum 35-Kuhbetrieb größer als bei den Vergleichsbetrieben zum 68-Kuhbetrieb, bedingt durch andere Betriebszweige und unterschiedlich hohe Nebenerlöse. 
Abbildung 6.6: $\quad$ Einordnung der IFCN-Betriebe in die FADN-Verteilung auf Basis der Produktionskosten - Betrieb D-35 (BY)

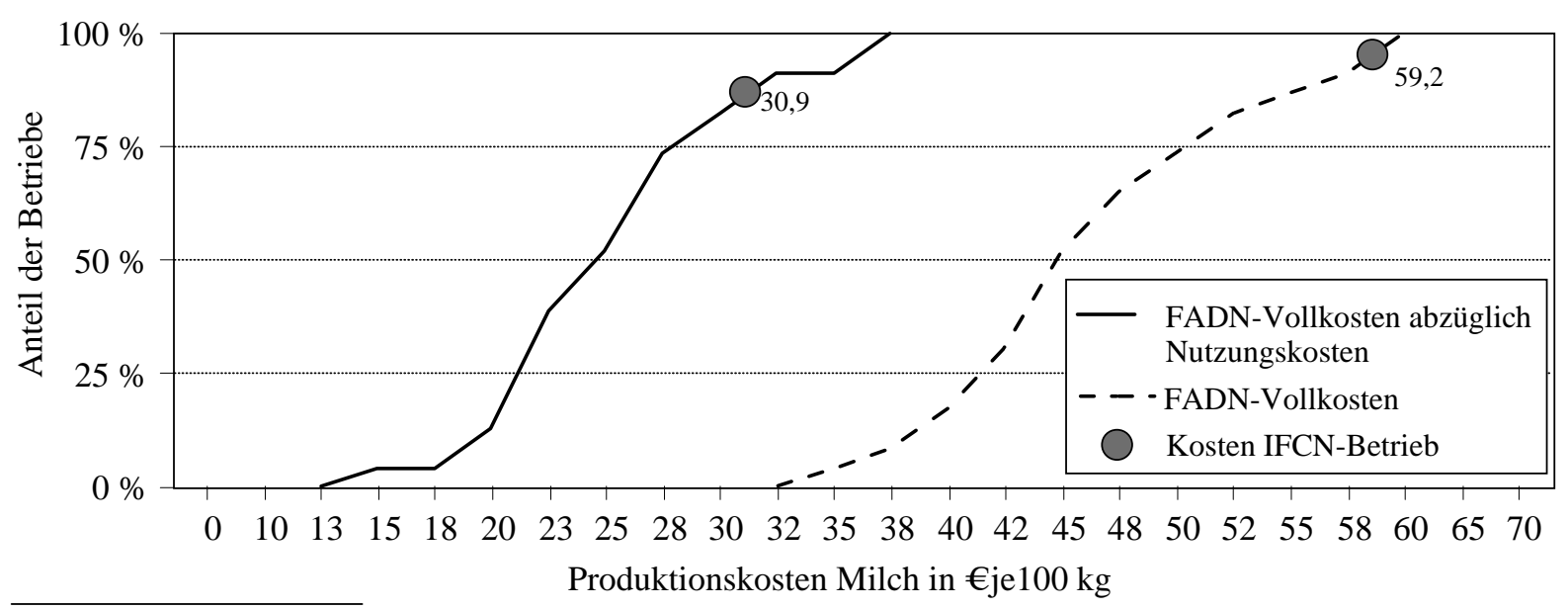

Quelle: Eigene Berechnung und Darstellung; Datenbasis: Europäisches Testbetriebsnetz (FADN, 1999/2000).

\section{Übertragung der IFCN-Analyseergebnisse}

Im Vordergrund steht nun die Frage, wie die zuvor ermittelten Ergebnisse bei der Interpretation von Analysen zum MTR genutzt werden. Da der IFCN 35-Kuhbetrieb aus Bayern bedeutend höhere Kosten aufweist als die zu vergleichenden FADN-Betriebe und das obere Viertel auf der Verteilungskurve widerspiegelt, lässt sich für die Betriebe aus der entsprechenden FADN-Stichprobe und die dadurch repräsentierten Betriebe aus der Grundgesamtheit Folgendes ableiten: a) Im Hinblick auf die prognostizierten Einkommenseinbußen zu Beginn der Umsetzung der MTR-Maßnahmen sind die Milchviehbetriebe aufgrund geringerer Produktionskosten insgesamt möglicherweise weniger ökonomisch gefährdet als für den IFCN 35-Kuhbetrieb angenommen; b) Die Anzahl der Betriebe, die womöglich ausscheiden werden, würde dementsprechend geringer ausfallen, als für den IFCNBetrieb prognostiziert.

Die ermittelten Einkommenswirkungen für den IFCN 68-Kuhbetrieb können mit Hilfe der Einordnung in die FADN-Verteilung gut auf eine größere Gruppe von Betrieben übertragen werden. Das bedeutet, dass die Ergebnisse tatsächlich eine größere Betriebsgruppe repräsentieren und dadurch fundierter sind. Beispielsweise kann die Aussage, dass mittel/langfristig für den 68-Kuhbetrieb aufgrund der MTR-Beschlüsse im Vergleich zur Agenda 2000 keine signifikanten Einkommenseinbußen zu erwarten sind (siehe Kapitel 5.2), bekräftigt werden. Wachstumsschritte (ohne Milchquote) würden den derzeitigen Produktionsanteil dieser Betriebsgruppe von ca. 4,9 auf 8,0\% an der regionalen Milchproduktion erhöhen. Damit könnte für diese Gruppe von Milchviehbetrieben, die ca. 2,7 bis 3,8 \% der Milchviehbetriebe in der Region repräsentieren, auch langfristig eine rentable Produktion gewährleistet sein. Hier ist nochmals zu erwähnen, dass die FADN-Vergleichsgruppen, bestehend aus jeweils 25 Vergleichsbetrieben, sehr eng gefasst sind. Wenn eine Erhöhung 
der Anzahl Vergleichsbetriebe zugelassen wird, vergrößert sich der abgebildete Ausschnitt aus der Grundgesamtheit, auf den die Ergebnisse übertragen werden können, allerdings zu Lasten der durchschnittlichen Ähnlichkeit der Vergleichsgruppe zum typischen Betrieb.

\subsubsection{Diskussion}

Das Ziel des ersten Verknüpfungsansatzes ist die statistische Einordnung der IFCNBetriebe mit Hilfe von FADN-Betriebsdaten. Die Einordnung erfolgte in einem zweistufigen Prozess. In der ersten Stufe wurden FADN- und IFCN-Daten aufgrund ihrer strukturellen Merkmale verglichen und mit Hilfe des euklidischen Distanzmaßes zwei FADNBetriebe ausgewählt, die den Untersuchungsbetrieben aus dem IFCN strukturell am ähnlichsten sind. Dabei wurde als Abschneidegrenze die Anzahl der Betriebe auf 25 begrenzt, um ein Mindestmaß an Ähnlichkeit zu erhalten. In der zweiten Stufe wurden die IFCNund ausgewählten FADN-Betriebe hinsichtlich ihrer wirtschaftlichen Leistungskennzahlen verglichen und eingeordnet. Unter Berücksichtigung der unterschiedlichen Effizienz der Betriebe aus dem FADN und IFCN wird geprüft, ob die Analyseergebnisse zum Mid-term Review aus dem IFCN generalisiert werden können.

Wie die Berechnungen zeigen, können im Hinblick auf die strukturellen Merkmale von sieben IFCN-Betrieben vier IFCN-Betriebe ${ }^{28}$ für die jeweilige Region als typisch eingestuft werden. Diese Betriebe reflektieren in den untersuchten Ländern in der Regel die durchschnittliche Betriebsgröße. Durch eine Variation bzw. eine Erweiterung der Region konnte die Ähnlichkeit zwischen FADN- und IFCN-Betrieb nochmals erhöht werden. ${ }^{29}$

${ }^{28} \quad$ Folgende Betriebe konnten als typisch eingestuft werden: der bayerische 35-Kuhbetrieb, der niedersächsische 68-Kuhbetrieb, der katalonische 42-Kuhbetrieb und der westschwedische 50-Kuhbetrieb.

29 Die Erweiterung der Stichprobe aus Niedersachsen auf die Region Schleswig-Holstein hat die Ähnlichkeit der FADN-Betriebsgruppe zum typischen IFCN-Betrieb nochmals verbessert. Dieselben Effekte konnten für den katalonischen 42-Kuhbetrieb durch Erweiterung der Stichprobe auf drei weitere Regionen in Nordspanien erzielt werden. 


\section{Anzahl Betriebe in der Vergleichsgruppe}

Am Beispiel der katalonischen Betriebsstichprobe zeigt sich, dass ein anderer Weg, beispielsweise die Wahl einer Ähnlichkeitszahl als Abschneidegrenze und nicht die festgelegte Anzahl von 25 Betrieben je Gruppe, möglicherweise der bessere Weg ist als der hier gewählte. Während die ersten Betriebe der katalonischen Vergleichsgruppe hinsichtlich der Ähnlichkeitszahl deutlich besser liegen als die nordspanische Vergleichsgruppe, weichen einige Betriebe im letzten Viertel der katalonischen Vergleichsgruppe deutlich ab. Dadurch verschlechtert sich die durchschnittliche Ähnlichkeitskennzahl der katalonischen Vergleichsgruppe. Die Gruppengröße sollte daher nicht von vornherein auf eine bestimmte Anzahl Betriebe festgelegt werden.

\section{Auswahl der IFCN-Betriebe}

Als offene Frage bleibt, ob bei der Bildung der Vergleichsgruppe ein anderes Auswahlverfahren zu besseren Ergebnissen führt. Beispielsweise wäre zu prüfen, ob die Setzung von absoluten und relativen Grenzen bei jedem Auswahlkriterium zu günstigeren Vergleichsgruppen führt als das Heranziehen der kumulierten euklidischen Distanz. Dadurch könnten die Abweichungen bei den bedeutendsten Kriterien (z. B. Milchviehbetriebe, Anzahl Milchkühe, Milchquote) relativ gering gehalten werden. Gegebenenfalls umfassen diese Gruppen eine größere Anzahl von Betrieben und würden dann mehr Betriebe aus der Grundgesamtheit repräsentieren. Ein weiterer Aspekt besteht darin, die Managementfähigkeit der Betriebe bei der Selektion der FADN-Vergleichsgruppen zu berücksichtigen. Zu diesen Fragen besteht weiterer Untersuchungsbedarf.

\section{Effizienzvergleich von FADN- und IFCN-Betrieben}

Auf Basis des Effizienzvergleichs von FADN- und IFCN-Betrieben konnte gezeigt werden, dass FADN dabei helfen kann, die IFCN-Betriebe hinsichtlich ihrer Wirtschaftlichkeit einzuordnen und zu überprüfen, wie typisch die IFCN-Betriebe für ihre Größenklasse in der Region und im Land sind. Dafür wurden als Indikatoren die Vollkosten und die Kosten aus der Gewinn-und-Verlust-Rechnung herangezogen.

\section{Hochrechnungsmöglichkeit mit Hilfe von FADN}

Es konnte gezeigt werden, welchen Anteil die IFCN-Betriebe aus der Grundgesamtheit abbilden. Der Anteil repräsentierter Betriebe aus der Grundgesamtheit (2,6 bis 3,8\%) könnte durch eine größere Anzahl einbezogener Betriebe erhöht werden. Dann müsste jedoch eine ungünstigere Ähnlichkeitszahl und damit eine inhomogenere Vergleichsgruppe akzeptiert werden.

Die Repräsentativität des typischen niedersächsischen Betriebs bezogen auf die Grundgesamtheit erhöht sich beispielsweise auf acht bzw. $20 \%$, wenn anstelle von maximal 25 Betrieben 60 bzw. 150 FADN-Betriebe mit der höchsten Ähnlichkeit zum typischen 
IFCN-68-Kuhbetrieb zugeordnet werden. Das euklidische Distanzmaß erhöht sich dabei um 0,3 bzw. 0,6 Prozentpunkte und die Standardabweichung steigt erwartungsgemäß an.

\section{Übertragung von Analyseergebnissen}

Gezeigt werden konnte, dass FADN dabei helfen kann, den Rückschluss vom IFCNBetrieb zur Grundgesamtheit mit Hilfe der gewählten Vorgehensweise zu ermöglichen. Es konnte am Beispiel der Untersuchungsbetriebe dargelegt werden, für wie viele und für welche Betriebe aus der Grundgesamtheit die Ergebnisse aussagekräftig sind. Je nach dem, wie der IFCN-Betrieb im Hinblick auf seine wirtschaftliche Leistungsfähigkeit im Vergleich zur FADN-Betriebsgruppe einzuordnen ist, werden bei der Übertragung der IFCNAnalyseergebnisse auf die FADN-Betriebsgruppe die Ergebnisse entsprechend angepasst und justiert. Anhand der Einordnung wird deutlich, für wie viele Betriebe die Ergebnisse aus dem IFCN gelten können.

Darüber hinaus ermöglichen die Einordnung und der Vergleich der Daten typischer Betriebe mit den Struktur- und Leistungsdaten aus dem FADN eine Kontrolle und gegebenenfalls eine Korrektur (bzw. Neudefinition) der Inputdaten typischer Betriebe. FADN kann dabei helfen, IFCN-Modellergebnisse mittels Einordnung zu verbessern und zu entscheiden, was typisch ist.

Zusammenfassend lässt sich festhalten, dass die kombinierte Anwendung beider Datensätze sich als sinnvoll erwiesen hat und einen Zugewinn an Erkenntnissen ermöglicht. Bei der Weiterentwicklung von IFCN sollte der Schwerpunkt darin liegen, die ermittelten Ergebnisse durch die Einordnung der erhobenen Betriebe auf ein größeres Fundament zu stellen.

Letztendlich ist jedoch zu bedenken, dass die mit Hilfe der Einordnung ermittelte Repräsentanz der IFCN-Betriebe je nach gewählter Ähnlichkeitszahl stark variieren kann und es keine objektive Regel gibt, welches Distanzmaß gerade noch akzeptabel ist. Von daher stellt sich die Frage, ob der FADN-Datensatz nicht schon besser bei der Auswahl der typischen Betriebe genutzt werden sollte, als nachher festzustellen, wie repräsentativ die mit Hilfe von Beratern festgelegten typischen Betriebe tatsächlich sind. Deshalb wird abschließend in Kapitel 6.5 ein Verfahren vorgestellt, wie die Auswahl typischer IFCNBetriebe auf Basis von FADN-Daten erfolgen kann. 


\subsection{Auswahl von typischen IFCN-Betrieben mit Hilfe der Clusteranalyse}

In dieser Arbeit wurde untersucht, inwieweit Produktionsrichtung und -umfang der typischen IFCN-Betriebe, die im Rahmen der Panelerhebung von Beratern gewählt worden sind, mit der Grundgesamtheit einer Region bzw. eines Landes übereinstimmen. Dabei hat sich gezeigt, dass die Repräsentativität der IFCN-Betriebe je nach vorgegebener euklidischer Distanz nicht besonders hoch ist. Von daher stellt sich die Frage, ob es nicht ein anderes Verfahren zur Auswahl der typischen Betriebe gibt, welches zu einer besseren Repräsentativität führt. Im Hinblick auf die Weiterentwicklung des Verknüpfungsansatzes wird im Folgenden ein Verfahren vorgestellt, wie IFCN-Betriebe z. B. mittels Clusteranalyse aus dem FADN gewählt werden können.

Unter Clusteranalyse wird ein Verfahren zur Klassifikation von Objekten verstanden. Die durch mehrere Merkmale beschriebenen Objekte werden in Gruppen (Cluster) eingeteilt (Steinhausen und Langer (1977), Deichsel (1985), BorTZ (1993)). Objekte einer Stichprobe werden systematisch in mehrere Cluster (Gruppen von Beobachtungen) so zusammengefasst, dass sie intern möglichst homogen und untereinander möglichst gut unterscheidbar sind. Die durch ihre Eigenschaften und Eigenschaftsausprägungen (im vorliegenden Fall naturale und mönetäre Daten landwirtschaftlicher Betriebe) beschriebenen Objekte (landwirtschaftliche Betriebe) werden je nach ihrer Nähe zueinander in Gruppen eingeteilt (SCHULZE PALS, 1994).

Das methodische Vorgehen bei der Clusteranalyse lässt sich in folgende Schritte einteilen:

- Zielsetzung der Clusteranalyse

- Definition der Stichprobe

- Auswahl der Klassifizierungsmerkmale

- Auswahl des geeigneten Clusterverfahrens und Distanzmaßes zur Gruppierung

- $\quad$ Technische Durchführung und Ergebnisse der Selektion

\section{Zielsetzung der Clusteranalyse}

Ziel der Analyse der FADN-Stichprobe ist es herauszufinden, welche Anzahl IFCNBetriebe pro Land nötig ist, um in der Vielfalt der Strukturen und Betriebskonstellationen die bedeutendsten Betriebstypen im Hinblick auf die Produktionsmenge abzudecken. Aus den gebildeten Clustern soll abgeleitet werden können, welche Strukturvorgaben für die typischen IFCN-Betriebe zu treffen sind.

Die Methodik der Clusteranalyse soll am Beispiel deutscher Milchviehbetriebe aus dem FADN-Datensatz aufgezeigt werden. 


\section{Stichprobe}

Die Datenbasis zur Analyse der Grundgesamtheit, die alle relevanten Betriebe umfasst, z. B. auf Basis von Daten der Betriebsstrukturerhebung des Statistischen Bundesamtes, steht im Rahmen dieser Arbeit nicht zur Verfügung. Daher wird hier die FADNStichprobe exemplarisch analysiert, die eine Teilmenge der Grundgesamtheit darstellt und die untersuchungsrelevanten Eigenschaften der Grundgesamtheit gut abbildet (vgl. BORTZ, 2005). In Anlehnung an die Zielsetzung, Typisierungen für IFCN-Betriebe zu finden, beschränkt sich die Stichprobe in dieser Beispielrechnung auf spezialisierte Milchviehbetriebe (Anteil der Milcherlöse an den Gesamterlösen des Betriebes > $60 \%$ ). Während in Westdeutschland die Milch größtenteils in spezialisierten Milchviehbetrieben erzeugt wird, wird in Ostdeutschland ein großer Anteil Milch in Betrieben erzeugt, die nicht als spezialisierte Milchviehbetriebe klassifiziert werden, deren Betriebszweig Milch jedoch eine vergleichsweise bedeutende Produktionsmenge umfasst. Daher wird die Auswahl spezialisierter Milchviehbetriebe in Ostdeutschland um alle Betriebe mit Milchkühen ergänzt. Die Stichprobe umfasst ca. 1.730 FADN-Betriebe, die etwa 70 \% der Milchproduktion in Deutschland repräsentieren. ${ }^{30}$

\section{Auswahl des geeigneten Clusterverfahrens und Distanzmaßes zur Gruppierung}

Die verschiedenen Clusteranalysemethoden unterscheiden sich hinsichtlich der Distanzfunktion zwischen den Clustern und zusätzlich in dem zu optimierenden Fusions- und Zielkriterium. Während bei einigen Verfahren beliebige Distanzen zwischen den Objekten zugelassen sind (Single Linkage, Complete Linkage), sind bei anderen die verwendbaren Distanzmaße zwischen Objekten vorgegeben. Welches Clusterverfahren anzuwenden ist, hängt von der Struktur des zur Verfügung stehenden Datensatzes und von dem gewünschten Gruppierungsergebnis ab. Grundsätzlich unterscheidet man zwischen hierarchischen (u. a. Single-Linkage und Ward-Verfahren) und nicht hierarchischen Verfahren (u. a. iteratives Minimaldistanzverfahren). Während das Klassifikationsergebnis von hierarchischen Verfahren (agglomerative $^{31}$ und divisive ${ }^{32}$ Verfahren) in der hierarchischen Anordnung der

Bei den zu untersuchenden FADN-Daten handelt es sich demzufolge um eine große Stichprobe, die nur landwirtschaftliche Betriebe einschließt, die als Haupterwerbsbetriebe gelten und eine bestimmte wirtschaftliche Betriebsgröße überschreiten. Es handelt sich nicht um eine uneingeschränkte Zufallsstichprobe, da nicht alle Betriebe die gleiche Wahrscheinlichkeit besitzen, in die Zufallsstichprobe aufgenommen zu werden (vgl. BORTZ, 2005). Zum einen beinhaltet die FADN-Stichprobe nur die Betriebe, die eine geeignete Betriebsbuchführung haben und zum anderen erfolgt die Teilnahme im Testbetriebsnetz auf freiwilliger Basis (siehe Kapitel 4.2.3).

Agglomerative Clusterverfahren: Alle Beobachtungen liegen zunächst isoliert vor. Schrittweise werden nach Maßgabe des gewählten Proximitätsmaßes einzelne Beobachtungen zu Gruppen zusammengefasst, die schrittweise zu immer größeren Gruppen aggregiert werden (SCHUlZE PALS, 1994).

Divisive Clusterverfahren: Alle Beobachtungen sind in der Ausgangssituation in einer Klasse vereinigt. Sukzessive werden immer kleinere Gruppen gebildet, bis schließlich jede Beobachtung isoliert vorliegt(SCHULZE PALS, 1994). 
Cluster auf unterschiedlichen Distanz- und Ähnlichkeitsebenen erfolgt, ist bei nicht hierarchischen Verfahren eine Anfangsgruppierung der Beobachtungen erforderlich, wobei die Homogenität der Anfangsgruppierung durch Verlagerungen von Beobachtungen in andere Gruppen verbessert wird.

Die im Rahmen dieser Arbeit zur Verfügung stehenden einzelbetrieblichen FADN-Daten befinden sich vor Beginn der Clusteranalyse in einer großen noch undifferenzierten Gruppe im FADN-Datenpool. Das Ziel dieser Clusteranalyse ist es, diese undifferenzierte Gruppe nach geeigneten Merkmalen in eine begrenzte Anzahl homogener Gruppen von Milchviehbetrieben einzuteilen. Daher wird die hierarchisch divisive Klassifikation nach Ward verwendet. Die große Anfangsstichprobe wird anhand ausgewählter Variablen hierarchisch in immer kleinere Teilgruppen unterteilt. Das Ward-Verfahren bildet Cluster mit annähernd gleichen Besetzungszahlen ab, die empfindlich gegen Ausreißer sind (MILLIGAN, 1980). Es enthält nicht Distanzen zwischen Elementen, sondern jeweils den Zuwachs an Heterogenität bei ihrer Verschmelzung. Es wird versucht, eine möglichst homogene Gruppierung dadurch zu konstruieren, dass in jedem Schritt diejenigen Gruppen zusammengefasst werden, die den geringsten Zuwachs zu einem Heterogenitätsmaß (Varianzkriterium $^{33}$ ) liefern. Die Streuung innerhalb des Clusters wird minimiert.

Das Ward-Verfahren ist auch bekannt unter der Bezeichnung Minimum-Varianz-Methode bzw. Fehlerquadratsummenmethode oder HGROUP 100-Methode. Ausgangspunkt dieses Verfahrens ist eine Datenmatrix, die für jedes Objekt p Merkmale enthält. Diese Methode beruht auf einer Clustervorstellung, bei der die Clusterzentren als Repräsentanten betrachtet werden. Es wird angenommen, dass ein Cluster durch sein Zentrum repräsentiert werden kann. Diesen Punkt erhält man, indem für jedes Merkmal aus den Einzelwerten der Objekte des Clusters der Mittelwert bestimmt wird. Als hierarchisches Verfahren folgt der Ward-Algorithmus also dem folgenden Vorgehen:

1. Schritt: Jedes Objekt ist ein Cluster und gleichzeitig Clusterzentrum.

2. Schritt: Gesucht wird das Clusterpaar $\mathrm{C}_{x}, \mathrm{C}_{y}$, das bei einer Verschmelzung zu einer minimalen Zunahme der Abstandsquadratsumme in den Clustern führt.

3. Schritt: Das Clusterpaar $\mathrm{C}_{x}, \mathrm{C}_{y}$ y wird verschmolzen, die Clusterzentren werden neu berechnet (Mittelwerte über alle Elemente im neuen Cluster).

4. Schritt: Die Schritte 2 und 3 werden so lange wiederholt, bis alle Objekte einem einzigen Cluster angehören. 
Die Unähnlichkeit (Distanz) zwischen Objekten entspricht bei dem Ward-Verfahren der euklidischen Distanz. Bei unterschiedlichen Maßstäben der verwendeten Merkmale ist eine Standardisierung vor der Clusteranalyse empfehlenswert.

Sollten zwei Clusterpaare den gleichen Abstand haben, so entscheiden die Besetzungszahlen über die Fusionierung. Mit ersten Fusionsschritten werden bevorzugt kleine Cluster in Regionen mit hoher Objektdichte gebildet. Mit fortschreitender Fusionierung tendiert der Algorithmus dazu, Unterschiede in den Besetzungszahlen der Cluster auszugleichen. Es werden dann Cluster mit annähernd gleichgroßen Besetzungszahlen gebildet.

Angenommen, man hätte eine Zerlegung G mit k Clustern. Die Zahl der Objekte im Cluster i sei mit $n_{i}$ (vgl. BoRTZ, 2005) bezeichnet. Dann hat das Varianzkriterium folgende Form (k Cluster, p Merkmale, n Objekte im Cluster):

$$
Z_{q s}(G)=\sum_{i=1}^{k} \sum_{j=1}^{p} \sum_{m=1}^{n}\left(x_{i j m}-\bar{x}_{i j}\right)^{2}
$$

Mi Hilfe des Ward-Algorithmus wird eine Zerlegung G gesucht, für die das Varianzkriterium $\mathrm{Z}_{q s}(\mathrm{G})$ minimal wird. Bei Fusion zweier Cluster $\mathrm{C}_{x}$ und $\mathrm{C}_{y}$ erhält man die Differenz der Fehlerquadratsumme aus der Formel

$$
\Delta Z_{q s}(G)=n_{x} n_{y} \sum_{j=1}^{p}\left(\vec{x}_{x j}-\vec{x}_{y j}\right)^{2} /\left(n_{x}+n_{y}\right),
$$

wobei $\mathrm{n}_{x}$ und $\mathrm{n}_{y}$ jeweils die Anzahl der Elemente in Cluster $\mathrm{C}_{x}$ bzw. $\mathrm{C}_{y}$ bezeichnet.

Die Minimierung der Abstandsquadratsummenzuwächse ist gleichbedeutend mit der Minimierung der quadrierten euklidischen Distanz der Schwerpunkte der zu fusionierenden Cluster.

Obwohl das Verfahren primär für quadrierte euklidische Distanzen zwischen Objekten entwickelt worden ist, lässt es sich auch auf andere Distanzfunktionen mit zufriedenstellenden Ergebnissen anwenden (Steinhauser, LANGer, 1977). Darauf wird jedoch im Rahmen dieser Arbeit verzichtet.

Das Ergebnis dieses Clusterverfahrens lässt sich z. B. in Form eines hierarchisch aufgebauten Baumdiagramms darstellen (siehe Abbildung 6.7). 
Abbildung 6.7: $\quad$ Konstruktionsprinzip bei der hierarchischen Klassifikation

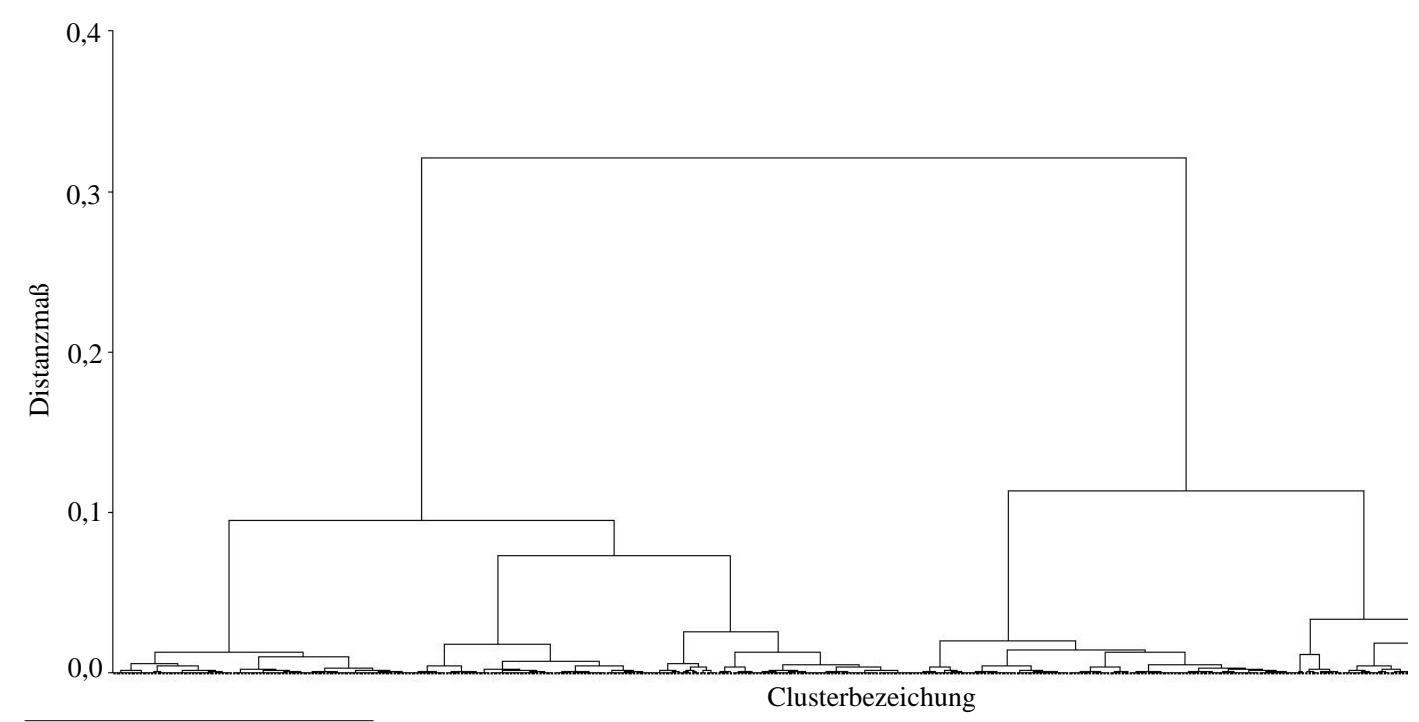

Quelle: Eigene Darstellung auf Basis von INLB-EU-GD-AGRI/G3.

In Abbildung 6.7 wird die hierarchisch divisive Klassifikation nochmals deutlich. Abbildung 6.7 zeigt einen Ausschnitt gebildeter Cluster. Die Baumstruktur verästelt sich weiter, so dass jedes Cluster einzeln dargestellt wird. Bei einem Distanzmaß von 0,03 ergeben sich beispielsweise 5 Cluster.

\section{Auswahl der Klassifizierungsmerkmale}

Die Auswahl geeigneter Selektionsvariablen orientiert sich an der Zielsetzung, Gruppen von Milchviehbetrieben zu bilden, die die Vielfalt der Strukturen und Betriebskonstellationen möglichst gut reflektieren und eine bedeutende Produktionsmenge abdecken. Da die Klassifizierung sehr stark abhängig ist von der Zahl der Variablen, wird die Anzahl berücksichtigter Variablen auf einige wenige begrenzt. Eine große Anzahl von Variablen führt zu Überlappungen zwischen den Clustern. Als Ergebnis erhält man in der Regel wenig differenzierte Cluster. Die Gewichtung ist aufgrund der Fülle an Daten oft wenig aussagekräftig.

Als regionale Abgrenzung wurde Deutschland in drei Regionen untergliedert:

- Region 1 (Nord-West): Schleswig-Holstein, Niedersachsen, Nordrhein-Westfalen

- Region 2 (Mitte-Süd): Übrige Bundesländer im alten Bundesgebiet

- $\quad$ Region 3 (Ost): Neue Bundesländer. 
Als ein Klassifizierungsmerkmal wurde in Anlehnung an die Zielsetzung die Betriebsgröße gewählt. Hier werden folgende Selektionsvariablen berücksichtigt:

- Landwirtschaftlich genutzte Fläche (Hektar)

- Zahl der Milchkühe (Anzahl)

- Standarddeckungsbeitrag des Gesamtbetriebes (Euro/Betrieb).

Die Intensität der Milchproduktion soll durch den Viehbesatz widergespiegelt werden:

- Milchvieh GV-Einheiten je Hektar Hauptfutterfläche

Die Naturalleistung und der wirtschaftliche Erfolg sollen die Managementfähigkeiten des Betriebes reflektieren. Folgende Variablen werden gewählt:

- Milchleistung pro Kuh (kg/Kuh und Jahr)

\section{Technische Durchführung und Ergebnisse der Selektion}

Das beschriebene Clusterverfahren wird auf die ausgewählten 1.730 Milchviehbetriebe aus dem deutschen FADN angewendet. Die Berechnung erfolgt mit dem Statistikprogramm SAS. Die Gewichtung jedes Einzelbetriebs erfolgt mit dem entsprechenden Hochrechnungsfaktor aus dem FADN-Datenpool.

Um zu einer Entscheidung zu kommen, wie viele Cluster maximal gebildet werden sollen, wurden verschiedene Vorgaben getestet. Dabei wurde festgestellt, dass beispielsweise bei einer Begrenzung auf 50 Cluster kaum noch Differenzierungen vorlagen. Bei einer Begrenzung auf fünf Cluster war die Homogenität der Cluster nicht mehr gegeben. Letztendlich ergab eine maximale Begrenzung auf 15 Cluster eine Gruppierung von zwölf Clustern je Region, die relativ detailgenaue Werte lieferten.

Die Ergebnisse der angewendeten Methodik zur Gruppierung der Milchviehbetriebe aus dem FADN sind in den Tabellen 6.11 bis 6.14 dargestellt. Darin sind die Cluster je Region mit ihren Besatzgrößen, den Mittelwerten der „Cluster-Variablen“ und ergänzenden erklärenden Variablen sowie mit dem prozentualen Anteil des jeweiligen Clusters an der Milcherzeugung der Grundgesamtheit (in Deutschland) dargestellt (weitere Angaben siehe Anhang A6.8 bis A6.11). 
Tabelle 6.11: $\quad$ Ergebnisse der Selektion in der Region Nord-West-Deutschland

\begin{tabular}{|c|c|c|c|c|c|c|c|c|c|c|c|c|}
\hline \multirow[t]{2}{*}{ Region ") } & \multirow{2}{*}{$\begin{array}{c}\text { Milchvieh- } \\
\text { betriebe GG }\end{array}$} & \multicolumn{2}{|c|}{$\begin{array}{c}\text { Cluster-Variablen } \\
\qquad \mathbf{L F}^{3}\end{array}$} & \multicolumn{2}{|c|}{ Milchkü he ${ }^{6)}$} & \multicolumn{2}{|c|}{ Milchleist ung ${ }^{\text {’ }}$} & \multicolumn{2}{|c|}{ MvHffl $^{8)}$} & \multicolumn{2}{|c|}{ StDB $^{9)}$} & \multirow{2}{*}{$\begin{array}{c}\% \text { Anteil } \\
\text { MP GG-DE }\end{array}$} \\
\hline & & MW $^{4)}$ & STD $^{5)}$ & MW & STD & MW & STD & MW & STD & MW & STD & \\
\hline 1 & 2114 & 32 & 61 & 23 & 41 & 5805 & 4775 & 0,8 & 1,3 & 46 & 90 & 1,5 \\
\hline 1 & 352 & 35 & 39 & 26 & 62 & 3394 & 3176 & 0,9 & 1,2 & 52 & 94 & 0,2 \\
\hline 1 & 1432 & 32 & 88 & 35 & 85 & 6248 & 4742 & 1,6 & 2,1 & 66 & 165 & 1,8 \\
\hline 1 & 2752 & 52 & 87 & 41 & 67 & 8187 & 3669 & 0,9 & 1,1 & 84 & 137 & 5,1 \\
\hline 1 & 2914 & 65 & 100 & 47 & 70 & 6700 & 2281 & 0,8 & 1,1 & 96 & 136 & 5,1 \\
\hline 1 & 1023 & 40 & 68 & 48 & 64 & 8519 & 3639 & 1,6 & 1,1 & 93 & 128 & 2,3 \\
\hline 1 & 1455 & 63 & 74 & 56 & 78 & 5282 & 3463 & 1,0 & 1,3 & 109 & 124 & 2,4 \\
\hline 1 & 2877 & 77 & 98 & 70 & 74 & 8017 & 3856 & 1,2 & 1,4 & 138 & 114 & 9,0 \\
\hline 1 & 764 & 54 & 101 & 73 & 106 & 7633 & 4568 & 2,3 & 2,0 & 138 & 159 & 2,4 \\
\hline 1 & 1337 & 106 & 174 & 85 & 53 & 6304 & 5428 & 1,0 & 1,3 & 175 & 142 & 3,9 \\
\hline 1 & 1172 & 113 & 158 & 106 & 116 & 8059 & 5892 & 1,2 & 1,5 & 211 & 172 & 5,6 \\
\hline 1 & 649 & 172 & 256 & 150 & 229 & 7405 & 5926 & 1,1 & 2,7 & 304 & 262 & 4,0 \\
\hline
\end{tabular}

1) Region: 1 = Nord- $\mathrm{u}$. Westdeutschl and, 2= Süddeutsch land, 3 = Ostdeutschland. 2) Milchviehbetrieb e GG: Von FADN-Betrieben repräsentierte Anzahl Milchviehbetriebe in der Grundges am theit in Deuts chland. 3) LF (ha): Land wirtschaftliche Nutzfläche in Hektar(SE 025). 4) MW: Mittelwert. 5) STD: Standardabweichung. 6) Milchkühe: Anzahl Milchkühe (St ückzahl) (SE 085). 7) Milchleistung in Kilo gramm (SE 125). 8) MvHffl: Milchviehgroß vieheinheiten je Hek ta Hauptfutterflädhe. 9) StDB: Stan darddeckungsb eitrag des Ges am tbetriebes in 1000 Euro (A 27). 10)\%-Ant eil MP GG-DE: Anteil der erzeugten Mi lch in der Grundgesamtheit in Deuts chland in Prozent.

Quelle: Eigene Berechnungen. INLB-EU-GD AGRI/G3

Tabelle 6.12: $\quad$ Ergebnisse der Selektion in der Region Mitte- und Süddeutschland

\begin{tabular}{|c|c|c|c|c|c|c|c|c|c|c|c|c|}
\hline \multirow[t]{2}{*}{ Region ${ }^{1)}$} & \multirow{2}{*}{$\begin{array}{c}\text { Milchvieh- } \\
\text { betriebe GG }\end{array}$} & \multicolumn{2}{|c|}{$\begin{array}{c}\text { Cluster-Variablen } \\
\qquad \mathbf{L F}{ }^{3}\end{array}$} & \multicolumn{2}{|c|}{ Milchkühe ${ }^{6)}$} & \multicolumn{2}{|c|}{ Milchleist ung ${ }^{\eta}$} & \multicolumn{2}{|c|}{ MvHffl $^{8)}$} & \multicolumn{2}{|c|}{ StDB $^{9)}$} & \multirow{2}{*}{$\begin{array}{c}\text { \% Anteil } \\
\text { MP GG-DE }{ }^{10)}\end{array}$} \\
\hline & & $\mathrm{MW}^{4)}$ & STD $^{5)}$ & MW & STD & MW & STD & MW & STD & MW & STD & \\
\hline 2 & 4378 & 29 & 64 & 22 & 40 & 4370 & 5335 & 1,0 & 1,7 & 41 & 61 & 2,4 \\
\hline 2 & 6431 & 31 & 87 & 23 & 44 & 5508 & 3510 & 0,9 & 1,6 & 43 & 74 & 4,5 \\
\hline 2 & 6564 & 26 & 53 & 24 & 46 & 7000 & 4693 & 1,1 & 1,4 & 44 & 80 & 6,2 \\
\hline 2 & 1650 & 48 & 128 & 25 & 46 & 7670 & 4120 & 0,6 & 1,0 & 51 & 100 & 1,8 \\
\hline 2 & 8116 & 23 & 54 & 27 & 51 & 5954 & 5308 & 1,5 & 1,9 & 46 & 87 & 7,3 \\
\hline 2 & 2060 & 35 & 105 & 42 & 64 & 6855 & 6346 & 1,9 & 2,3 & 73 & 133 & 3,3 \\
\hline 2 & 1270 & 57 & 101 & 43 & 59 & 4436 & 2642 & 1,2 & 1,7 & 84 & 106 & 1,3 \\
\hline 2 & 4743 & 57 & 91 & 43 & 43 & 6762 & 6221 & 1,0 & 1,2 & 83 & 82 & 7,6 \\
\hline 2 & 2365 & 62 & 89 & 57 & 47 & 6464 & 3384 & 1,4 & 1,4 & 106 & 84 & 4,8 \\
\hline 2 & 1620 & 110 & 109 & 66 & 61 & 6521 & 5427 & 0,9 & 0,9 & 135 & 86 & 3,9 \\
\hline 2 & 1173 & 88 & 101 & 85 & 73 & 7052 & 4838 & 1,5 & 1,5 & 163 & 116 & 3,9 \\
\hline 2 & 433 & 171 & 137 & 115 & 152 & 7602 & 4682 & 1,2 & 1,3 & 247 & 236 & 2,1 \\
\hline
\end{tabular}

1) Region: $1=$ Nord- $\mathrm{u}$. Westdeutschl and, 2= Süddeutsch land, 3 = Ostdeutschland. 2) Milchviehbetrieb e GG: Von FADN-Betrieben repräsentierte Anzahl Milchviehbetriebe in der Grundges am theit in Deuts chland. 3) LF (ha): Land wirtschaftliche Nutzfläche in Hektar (SE 025). 4) MW: Mi ttelwert. 5) STD: Standardabweichung. 6) Milchkühe: Anzahl Milchkühe (St ückzahl) (SE 085). 7) Milchleistung in Kilo gramm (SE 125). 8) MvHffl: Milchviehgroß vieheinheiten je Hek ta Hauptfutterfläd e. 9) StDB: Stan darddeckungsb eitrag des Ges antbetriebes in 1000 Euro (A 27). 10) \%-Ant eil MP GG-DE: Anteil der erzeugten Mi lch in der Grundgesamtheit in Deuts chland in Prozent

Quelle: Eigene Berechnungen. INLB-EU-GD AGRI/G3. 
Tabelle 6.13: $\quad$ Ergebnisse der Selektion in der Region D-Ost (spez. Milchviehbetriebe)

\begin{tabular}{|c|c|c|c|c|c|c|c|c|c|c|c|c|}
\hline \multirow[t]{2}{*}{ Region ${ }^{1)}$} & \multirow{2}{*}{$\begin{array}{c}\text { Milchvieh- } \\
\text { betriebe GG }{ }^{2)}\end{array}$} & \multicolumn{2}{|c|}{$\begin{array}{c}\text { Cluster-Variablen } \\
\qquad \text { LF }^{3}\end{array}$} & \multicolumn{2}{|c|}{ Milchkü he ${ }^{6)}$} & \multicolumn{2}{|c|}{ Milchleist ung ${ }^{\nearrow}$} & \multicolumn{2}{|c|}{ MvHffl $^{8)}$} & \multicolumn{2}{|c|}{ StDB ${ }^{9)}$} & \multirow{2}{*}{$\begin{array}{c}\text { \% Anteil } \\
\text { MP GG-DE }\end{array}$} \\
\hline & & MW ${ }^{4)}$ & STD ${ }^{5)}$ & MW & STD & MW & STD & MW & STD & MW & STD & \\
\hline 3 & 339 & 131 & 274 & 49 & 88 & 4790 & 2316 & 0,7 & 0,6 & 122 & 236 & 0,4 \\
\hline 3 & 214 & 90 & 121 & 65 & 74 & 5930 & 2573 & 1,6 & 0,5 & 144 & 172 & 0,5 \\
\hline 3 & 322 & 158 & 234 & 66 & 87 & 7520 & 1372 & 0,8 & 0,4 & 170 & 233 & 0,9 \\
\hline 3 & 435 & 138 & 317 & 75 & 158 & 6376 & 1147 & 1,1 & 0,5 & 176 & 358 & 1,1 \\
\hline 3 & 475 & 142 & 257 & 91 & 161 & 8168 & 1951 & 1,3 & 0,5 & 206 & 351 & 2,0 \\
\hline 3 & 26 & 164 & 104 & 118 & 193 & 5180 & 2186 & 2,5 & 0,7 & 235 & 228 & 0,1 \\
\hline 3 & 121 & 144 & 261 & 125 & 243 & 7684 & 1932 & 2,3 & 0,8 & 272 & 522 & 0,6 \\
\hline 3 & 153 & 405 & 388 & 172 & 191 & 7833 & 1687 & 0,8 & 0,4 & 433 & 358 & 1,1 \\
\hline 3 & 92 & 1339 & 779 & 325 & 307 & 7027 & 2605 & 0,5 & 0,5 & 1036 & 782 & 1,2 \\
\hline 3 & 110 & 793 & 518 & 386 & 217 & 7842 & 2046 & 1,0 & 0,6 & 953 & 387 & 1,8 \\
\hline 3 & 57 & 1586 & 1359 & 771 & 289 & 7812 & 2334 & 1,0 & 0,9 & 2051 & 760 & 1,9 \\
\hline 3 & 22 & 1623 & 974 & 1388 & 309 & 7437 & 2232 & 1,7 & 1,0 & 3064 & 1526 & 1,2 \\
\hline
\end{tabular}

1) Region: 1= Nord- u. Westdeutschl and, 2= Süddeutschland, 3 = Ostdeutschland. 2) Milchviehbetrieb e GG: Von FADN-Betrieben repräsentierte Anzahl Milchviehbetriebe in der Grundges am theit in Deuts chland. 3) LF (ha): Land wirtschaftliche Nutzfläche in Hektar (SE 025). 4) MW: Mittelwert. 5) STD: Standardabweichung. 6) Milchkühe: Anzahl Milchkühe (Stückzahl) (SE 085). 7) Milchleistung in Kilo gramm (SE 125). 8) MvHffl: Milchviehgroß vieheinheiten je Hek ta Hauptfutterfläh e. 9) StDB: Stan darddec kungsb eitrag des Ges an tbetriebes in 1000 Euro (A 27). 10) \%-Anteil MP GG-DE: Anteil der erzeugten Milch in der Grundgesamtheit in Deuts chland in Prozent.

Quelle: Eigene Berechnungen. INLB-EU-GD AGRI/G3.

Tabelle 6.14: $\quad$ Ergebnisse der Selektion in der Region D-Ost (alle Betriebe mit Milchvieh)

\begin{tabular}{|c|c|c|c|c|c|c|c|c|c|c|c|c|}
\hline \multirow{3}{*}{ Region ${ }^{1)}$} & \multirow{3}{*}{$\begin{array}{c}\text { Milchvieh- } \\
\text { betriebe GG }\end{array}$} & \multicolumn{4}{|c|}{ Cluster-Variablen } & \multirow{2}{*}{\multicolumn{2}{|c|}{ Milchleistung ${ }^{\text {7) }}$}} & \multirow{2}{*}{\multicolumn{2}{|c|}{ MvHffl ${ }^{8)}$}} & \multirow{2}{*}{\multicolumn{2}{|c|}{ StDB $^{9)}$}} & \multirow{3}{*}{$\begin{array}{c}\% \text { Anteil } \\
\text { MP GG-DE }{ }^{14)}\end{array}$} \\
\hline & & \multicolumn{2}{|c|}{$\mathbf{L F}^{3)}$} & \multicolumn{2}{|c|}{ Milchkühe ${ }^{6}$} & & & & & & & \\
\hline & & MW $^{4)}$ & $\mathrm{STD}^{5)}$ & MW & STD & MW & STD & MW & STD & MW & STD & \\
\hline 3 & 432 & 132 & 247 & 41 & 76 & 4592 & 2420 & 0,7 & 0,7 & 121 & 198 & 0,3 \\
\hline 3 & 415 & 179 & 427 & 59 & 129 & 8124 & 1291 & 0,7 & 0,9 & 184 & 402 & 0,7 \\
\hline 3 & 241 & 113 & 240 & 65 & 86 & 6982 & 1432 & 1,9 & 0,8 & 177 & 356 & 0,4 \\
\hline 3 & 260 & 160 & 347 & 76 & 144 & 5211 & 1986 & 1,9 & 1,3 & 206 & 355 & 0,4 \\
\hline 3 & 1287 & 222 & 523 & 85 & 201 & 6638 & 1673 & 0,9 & 0,7 & 246 & 546 & 2,7 \\
\hline 3 & 520 & 155 & 297 & 92 & 170 & 8103 & 2084 & 1,3 & 0,5 & 221 & 385 & 1,4 \\
\hline 3 & 188 & 782 & 1458 & 244 & 465 & 7728 & 2038 & 2,4 & 1,1 & 1053 & 2000 & 1,3 \\
\hline 3 & 718 & 1137 & 774 & 283 & 311 & 7443 & 2173 & 0,9 & 0,9 & 1128 & 803 & 5,6 \\
\hline 3 & 279 & 1914 & 855 & 318 & 288 & 7540 & 2100 & 0,7 & 0,7 & 1898 & 995 & 2,5 \\
\hline 3 & 49 & 4259 & 1287 & 636 & 356 & 7908 & 2575 & 1,1 & 1,3 & 4475 & 1499 & 0,9 \\
\hline 3 & 273 & 2083 & 1560 & 651 & 452 & 7779 & 2304 & 1,1 & 1,1 & 2340 & 1394 & 5,2 \\
\hline 3 & 103 & 2562 & 2098 & 1353 & 607 & 8089 & 2481 & 1,6 & 1,2 & 3735 & 2076 & 4,2 \\
\hline
\end{tabular}

1) Region: 1= Nord- u. Westdeutschland, 2= Süddeutsch land, 3 = Ostdeutschland. 2) Milch viehbetriebe GG: Von FADN-Betrieben rep räsentierte An zahl Milchviehbetriebe in der Grundgesamtheit in Deutschland. 3) LF (ha): Landwirtschaftliche Nutzfläche in Hektar (SE 025). 4) MW: Mittelwert. 5) STD: Stand ardabweichung. 6) Milchkühe: Anzahl Milchkühe (Stückzahl) (SE 085). 7) Milch lei stung in Kilogramm (SE 125). 8) MvHffl: Milchviehgroßvieheinheiten je Hektar Hauptfutterfläche. 9) StDB: Standarddeckun gs beitrag des Gesamtbetriebes in 1000 Euro (A 27). 10) AK: Arbei tskrafteinhei ten (gesamt) (SE 010). 11) Mil cherlöse: \%-Anteil Milcherlöse aı 12) Viehbestand: Gesamter Viehbestand in Vieheinheiten (LU)(SE 080). 13) Hff: Hau ptfutterfläche in Hektar (SE 071). 10) \%-Anteil MP GG-DE: Anteil der erzeugten Quelle: INLB-EU-GD AGRI/G3; eigene Berechnungen. 
Die Mittelwerte dienen als Charakteristika zur Klassifizierung typischer IFCN-Betriebe. Jede Region umfasst zwölf Gruppen (Cluster). Sortiert man die zwölf Cluster nach der Herdengröße, so ergeben sich in der Region Nord-West (Schleswig-Holstein, Niedersachsen, Nordrhein-Westfalen) grob vier Gruppen mit jeweils zwei bis fünf Clustern: Gruppen von 23 bis 35 Kühen, 41 bis 56 Kühen, 70 bis 85 Kühen und 106 bis 150 Kühen. Sortiert man die zwölf Cluster in der Region Mitte-Süd (Hessen, Rheinland-Pfalz, Saarland, Baden-Württemberg, Bayern) nach der Herdengröße, so ergeben sich grob vier Gruppen mit jeweils ein bis fünf Clustern: Gruppen von 22 bis 27 Kühen, 42 bis 43 Kühen, 57 bis 85 Kühen und von etwa 115 Kühen. In Ostdeutschland zeigt die Selektion nach spezialisierten Milchviehbetrieben eine sehr heterogene Grundgesamtheit (siehe Tabelle 6.13). Sortiert man in Ostdeutschland nach allen Betrieben mit Milchkühen, so ergeben sich auf Basis der Herdengröße sechs Gruppen (siehe Tabelle 6.14): Gruppen von etwa 41 Kühen, 59 bis 76 Kühen, 85 bis 92 Kühen, 244 bis 318 Kühen, 636 bis 651 Kühen und von etwa 1.353 Kühen.

Tabellen 6.11 bis 6.14 veranschaulichen, dass die Cluster mit ähnlicher Herdengröße teilweise noch deutliche Unterschiede hinsichtlich der Naturalleistung (Milchleistung), Intensität der Produktion (Viehbesatzdichte) und Ausstattung mit Fläche zeigen. Einige Gruppen sind jedoch im Hinblick auf die Ausprägung der Selektionsvariablen auch recht homogen. Zudem dient die Clusteranalyse u. a. auch dazu, um sogenannte „Ausreißer“ zu identifizieren. Die Bedeutung der einzelnen Cluster im Hinblick auf die nationale Milchproduktion variiert z. B. in Nord-West-Deutschland zwischen 0,2 und $9 \%$ und die Anzahl repräsentierter Milchviehbetriebe variiert zwischen 350 und 2.900 Betrieben aus der Grundgesamtheit.

Legt man das IFCN-Erhebungskonzept zugrunde, wonach vornehmlich durchschnittlich große und Wachstumsbetriebe ausgewählt werden, so ergäben sich beispielsweise bei der Vorgabe, dass diese Betriebe einen Produktionsanteil von 5 bis $9 \%$ widerspiegeln sollen, drei Typisierungen, aus denen typische IFCN-Betriebe abgeleitet werden könnten. Da die Ableitung der nötigen Anzahl von IFCN-Betrieben geprägt ist von der subjektiven Einschätzung des beteiligten Wissenschaftlers, empfiehlt es sich, die Ergebnisse mit Beratern und Landwirten zu diskutieren und zu validieren (Kombinationsansatz).

Man könnte nun derart vorgehen, dass für alle hier ermittelten 36 Cluster ein typischer Betrieb gebildet wird. Diese Anzahl ließe sich jedoch nicht im IFCN umsetzen. Daher wird vorgeschlagen, die Cluster mit den höchsten regionalen Produktionsanteilen Milchviehspezialberatern vorzustellen, um dann zu einer Auswahl zu kommen, die die Regionen am besten charakterisieren. Die Clusteranalyse schafft eine gute Grundlage zur weiteren Spezifizierung der typischen Betriebe und sorgt für mehr Transparenz über die vorliegenden Betriebsstrukturen. 


\section{Verknüpfungsansatz II: Aufbereitung von FADN-Daten zur Nutzung im Modell TIPI-CAL}

Ziel des zweiten Verknüpfungsansatzes ist es zu untersuchen, wie der FADN-Datensatz mit Hilfe von Informationen aus dem IFCN so angereichert werden kann, dass er für einzelbetriebliche Simulationsrechnungen (z. B. mit dem Modell TIPI-CAL) nutzbar ist.

In Kapitel 7.1 wird das Konzept zur Verknüpfung von FADN- und IFCN-Datensatz zunächst im Überblick dargestellt. Anschließend wird in Kapitel 7.2 die methodische Grundlage beschrieben, mit der die FADN-Daten für Projektionsrechnungen ergänzt, auf Plausibilität überprüft und unter Beibehaltung der Annahmen für den IFCN-Betrieb (Preise, Mengen, betriebliche Anpassungsstrategie) in die Zukunft projiziert werden (u. a. Koeffizientenschätzansatz). Im Folgenden (Kapitel 7.3) wird die Leistungsfähigkeit der Verknüpfung von FADN-Daten mit dem Simulationsmodell TIPI-CAL am Beispiel einer Änderung der EU-Milchmarktpolitik getestet. Dies wird am Beispiel eines FADN-Betriebes aufgezeigt und erarbeitet, könnte grundsätzlich aber auch für viele Betriebe angewendet werden. Es werden eine Wachstumsstrategie und zwei Politikszenarien modelliert. Dabei wird überprüft, ob die Verknüpfung des FADN-Datensatzes mit dem TIPI-CAL-Modell zu verwertbaren Ergebnissen führt. Zeigen die FADN- und IFCN-Simulationsergebnisse ähnliche Verläufe, so ist dies ein Hinweis dafür, dass die Verknüpfung erfolgreich war. Abschließend (Kapitel 7.4) wird diskutiert, inwiefern mittels Verknüpfung Fortschritte zu erzielen sind und welche Perspektiven die Verknüpfung von FADN-Daten mit dem TIPICAL-Modell aufzeigt.

Ein Überblick über die verschiedenen Stufen der in dieser Arbeit vorgenommenen Auswertungen mit den FADN- und IFCN-Datensätzen ${ }^{1}$ findet sich im Anhang in Tabelle A7.1. Darin werden außerdem die in die Analysen einbezogenen Länder, Politiken und Betriebsstrategien sowie Untersuchungsperioden spezifiziert.

\subsection{Verknüpfungskonzept im Überblick}

Das europäische FADN enthält Buchführungsdaten von einer Vielzahl von Betrieben. Das Netz dient vorrangig dem Ziel der Einkommensrechnung. Der FADN-Datensatz ist EUweit harmonisiert und im statistischen Sinne repräsentativ für die Grundgesamtheit. Der Datensatz enthält nur die wichtigsten Produktions- und Leistungskennziffern. Detaillierte Daten zu den einzelnen Produktionsverfahren liegen nicht vor. Daher sind diese Datensät-

Die zum Abschluss (Kapitel 6.5) durchgeführte Clusteranalyse ist in der Tabelle allerdings nicht enthalten. 
ze weniger für Simulationsmodelle geeignet. IFCN kann durch Ergänzung und Korrektur der FADN-Variablenliste helfen, die Aussagekraft der FADN-Modellanwendungen zu verbessern, insbesondere im Hinblick auf

- die Produktionstiefe (physische Daten, Mengengerüst),

- die produktionstechnischen Koeffizienten,

- die Übertragung von Anpassungsstrategien (IFCN-Panelinformationen),

- die Projektion der Betriebe in die Zukunft (Simulationsrechnung).

Dazu wird der FADN-Datensatz mit Hilfe verschiedener Verfahren ergänzt und korrigiert, so dass er für Simulationsrechnungen im TIPI-CAL-Modell nutzbar gemacht werden kann.

Das Konzept zur Aufbereitung der FADN-Daten für Projektionsrechnungen im Simulationsmodell TIPI-CAL wird in Kapitel 7.1.1 erörtert.

\subsubsection{Konzept zur Modifikation von FADN-Daten zur Simulation im Modell TIPI-CAL}

Um FADN-Betriebe für Projektionsrechnungen beispielsweise mit Hilfe des TIPI-CALModells heranziehen zu können, muss der FADN-Datensatz um weitere Quellen ergänzt werden. Dies geschieht im Hinblick auf das Ziel dieser Arbeit vornehmlich mit Daten aus dem IFCN. Damit die zu ergänzenden Daten möglichst gut zu den FADN-Betrieben passen, erfolgt im empirischen Teil dieser Arbeit die Verknüpfung nur mit IFCN-Betrieben, die eine hohe Ähnlichkeit zu den zu simulierenden FADN-Betrieben aufweisen (s. o.).

Um die Fehlerrisiken bei der Ergänzung des FADN-Datensatzes zu reduzieren, kommt u. a. die Koeffizientenschätzmethode zur Anwendung, die in Kapitel 7.2 spezifiziert wird. Die Daten aus externen Quellen werden dabei so korrigiert, dass sie zu den im Betrieb Erhobenen passen.

Um die Modellierbarkeit und die Aussagekraft von FADN-Berechnungen im Rahmen der Politikfolgenanalyse - insbesondere in Bezug auf betriebliche Anpassungsreaktionen - zu verbessern, wird folgende Vorgehensweise gewählt:

1. Detaillierte Ergänzung und Korrektur der FADN-Variablen durch IFCN-Daten: Im ersten Schritt soll überprüft werden, wo es Verbesserungsmöglichkeiten in der FADN-Datenlage gibt. Beim Ergänzen der FADN-Variablenliste wird jede Variable 
auf der Eingabeseite $\left(\right.$ INP $^{2}$ ) im TIPI-CAL-Modell des IFCN dahingehend untersucht, ob die entsprechende Variable im FADN-Datensatz vorhanden ist. Wenn keine passende Variable im FADN-Datensatz vorhanden ist, wird sie mittels verschiedener Verfahren generiert. In dieser Arbeit wird diese Vorgehensweise am Beispiel eines typischen Betriebes, der eine hinreichend hohe Ähnlichkeit zum FADN-Vergleichsbetrieb aufweist, demonstriert.

2. Analyse von FADN-Betrieben mit Hilfe von Informationen aus dem IFCN-Panel: Als wichtigsten Aspekt geht es hierbei um die Übertragbarkeit von betrieblichen Anpassungsreaktionen auf den FADN-Vergleichsbetrieb.

3. Nutzung des IFCN-Netzwerkes von Betriebsleitern und Beratern (Strategien etc.) zur Verbesserung der FADN-Ergebnisse: Zur Verbesserung der FADN-Ergebnisse werden Informationen aus den IFCN-Panelsitzungen verwendet. Dies sind im Wesentlichen Expertenmeinungen über die Fortschreibung des FADN-Vergleichsbetriebes für den 10-jährigen Simulationszeitraum (Mengen- und Preisentwicklungen), produktionstechnische Koeffizienten und die im IFCN-Panelprozess ermittelte betriebliche Anpassungsstrategie.

\subsection{Methodisches Vorgehen bei der Simulation von FADN-Betrieben}

Im Rahmen der Verknüpfung von FADN- und IFCN-Datensatz werden im Folgenden methodische Fragen bei der Aufbereitung der FADN-Daten zur Projektion im TIPI-CAL Modell erläutert.

\subsubsection{Projektion von FADN-Betrieben}

Den methodischen Rahmen für die einzelbetriebliche Projektion von FADN-Betrieben bildet das Simulationsmodell ${ }^{3}$ TIPI-CAL. ${ }^{4}$

INP = „Input sheet“, sogenannte Eingabeseite im TIPI-CAL-Modell (vgl. HEMME, 2000).

3

Einige Betriebsmodelle/Mathematische Programmierungsmodelle wurden ursprünglich für betriebliche Planungsrechnungen entwickelt, und später erst zunehmend auf die Analyse von Politikänderungen ausgerichtet. Folglich haben sie eine ähnliche Grundstruktur. Im Zentrum des Modells steht in der Regel der landwirtschaftliche Betrieb oder der einzelne Landwirt, der sich an veränderte agrarpolitische Rahmenbedingungen anzupassen hat. Eine wünschenswerte Eigenschaft mathematischer Programmierung ist die Fähigkeit, die unterschiedlichen Typen von Produktionssystemen mit dem betrieblichen Entscheidungsverhalten zu kombinieren. Aufgrund unzureichender Datengrundlage über die zu modellierenden Systeme werden aber häufig nur aggregierte Durchschnittswerte verwendet. Dies führt zu deutlichem Informationsverlust, welches die Abschätzung von einzelbetrieblichem Anpassungsverhalten als Folge von Politikänderungen oftmals unpräzise werden lässt. Das TIPI-CAL-Modell wird angewendet, um zu versuchen, diesem Problem zu begegnen. 
Das TIPI-CAL Modell ist ein sehr differenziertes Modell, da es zur ProduktionskostenAnalyse entwickelt worden ist. Die FADN-Daten werden primär zur Einkommensanalyse erhoben. Daher benötigt man weitere Informationen, um mit FADN-Daten im TIPI-CAL Modell Berechnungen durchführen zu können. Für Analysen im TIPI-CAL Modell ist es notwendig, dass diejenigen Variablen, die im FADN nicht vorhanden sind, ergänzt werden. Die folgende Abbildung 7.1 stellt schematisch dar, wie dies erfolgt. Es wird unterschieden zwischen Variablen, die a) im FADN vorhanden sind, b) nicht im FADN vorhanden sind und z. B. durch die IFCN-Variable ersetzt werden und c) die sowohl aus dem IFCN-Datensatz, als auch aus dem FADN-Datensatz generiert werden.

Abbildung 7.1: $\quad$ Schematische Darstellung zur Erweiterung des FADN-Datensatzes für Simulationsrechnungen im TIPI-CAL-Modell

FADN

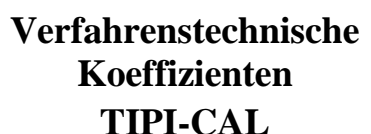

IFCN

TIPI-CAL

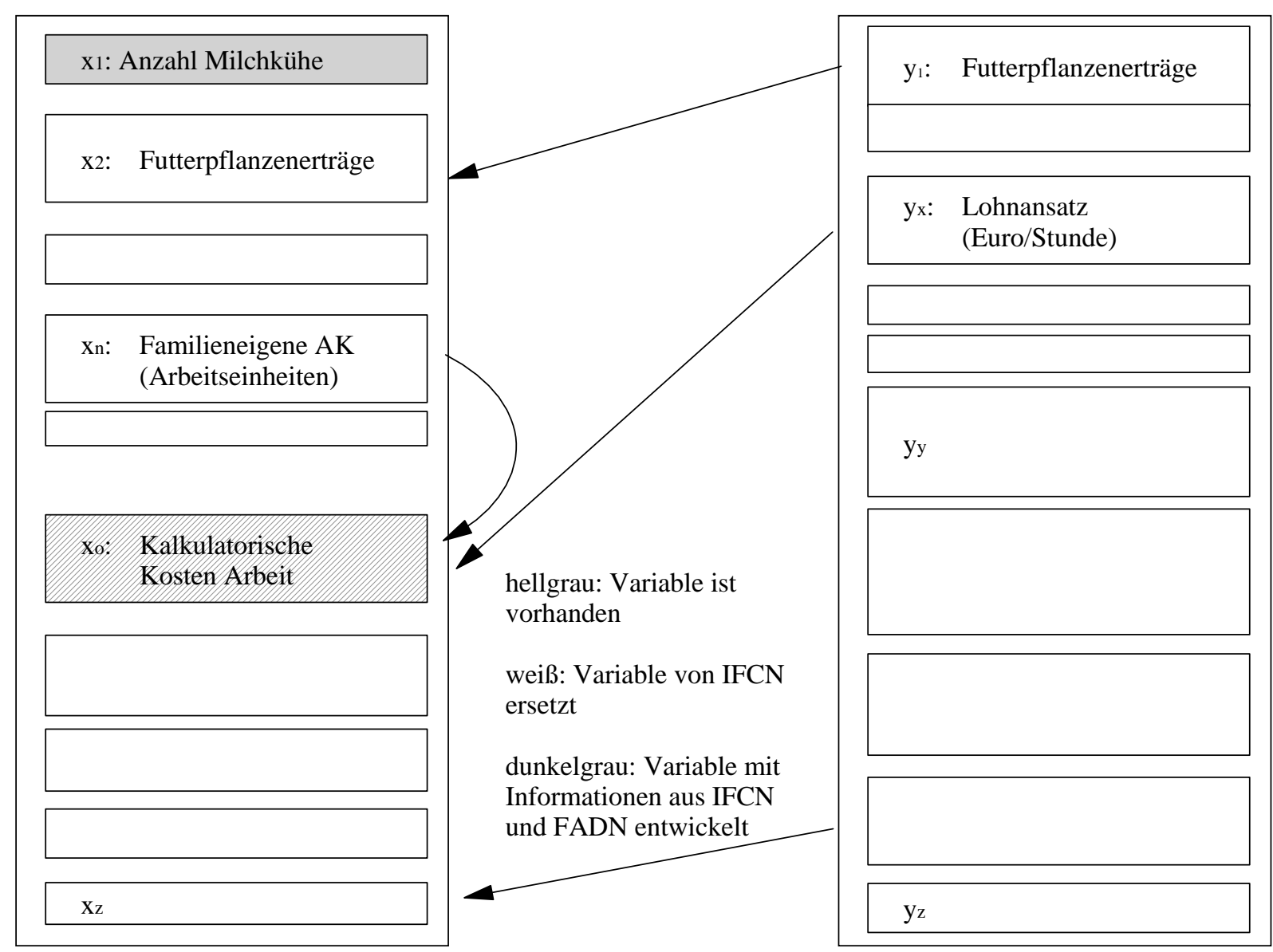

Quelle: Eigene Darstellung. 
Zur 10-jährigen Projektion von Betrieben bedarf es im TIPI-CAL-Modell einer Vorausschätzung von Preisen, Erträgen und Kostenentwicklungen. Die Annahmen ${ }^{5}$ zur Entwicklung der genannten Parameter basieren je nach Variable auf

- nationalen Statistiken (BMELV - z. B. Statistischer Monatsbericht, Statistischer Wochenbericht Vieh und Fleisch; ZMP - z. B. Marktbilanzen, Europamarkt Dauermilch, Bericht Schlachtvieh; Statistisches Bundesamt - z. B. Erzeugerdurchschnittspreise für inländisches Getreide; Fachzeitungen - z. B. Agrarwirtschaft),

- internationalen Statistiken (EUROSTAT New Cronos ${ }^{6}, 2002$; FAO; OECD-Dieselpreisentwicklung)

teilweise ergänzt durch (FAL ${ }^{7}$ ) -Expertenmeinungen (z. B. FAL-Modellverbund).

Die getroffenen Annahmen/Schätzungen werden kontinuierlich u. a. in Zusammenarbeit mit dem FAL-Modellverbund (KLEINHANSS et al., 2001; Kapitel 3.2.1 und 3.2.2 dieser Arbeit) überprüft und aktualisiert.

Die Grundlage der Politikanalyse bildet ein Referenzsystem, welches als „Baseline“ bezeichnet wird (vgl. Hemme, 2000). In dieser Arbeit wird im „Baseline“ Szenario unterstellt, dass die Milchquotenregelung unter den Bedingungen der Agenda 2000 fortgeführt wird. Diese Reform sieht eine Reduzierung der Milch-, Rindfleisch- und Getreidepreise vor. Die Preissenkungen werden kompensiert durch Ausgleichszahlungen je Hektar (63€/t Referenzertrag) für Getreide, Ölsaaten und Maissilage, Schlachtrinderprämien (100 €/Tier) und Ausgleichszahlungen je Kilogramm Milchquote $(2,5 € / 100 \mathrm{~kg}$ Milch in 2007 und Quotenaufstockung). Zudem wird angenommen, dass die Betriebsgröße und das Produktionssystem konstant bleiben. Die aufgrund von Milchleistungssteigerungen zusätzlich benötigte Milchquote wird zugekauft. Mit Hilfe dieser Datengrundlage wird auch der FADN-Betrieb in die Zukunft projiziert: Die FADN-Eingabedaten für das Jahr 1999 werden im Simulationsmodell TIPI-CAL mit den oben abgeleiteten Indizes bis zum Jahr 2008 fortgeschrieben. Die Annahmen sind für die IFCN- und FADN-Betriebe identisch.

Neben den Politik- und Marktszenarien wird an einem Beispiel auch die Übertragung einer Anpassungsstrategie aus der IFCN-Erhebung (IFCN-Panel) auf den FADN-Vergleichsbetrieb untersucht.

5 Die genauere Zuordnung der Quellen zu den abgeleiteten Variablen findet sich in der Anhangstabelle A7.2.

6 Thema 1 „Allgemeine Statistik“, Bereich „Regionalstatistiken“, Datenblock „Agrarstatistik“.

7 Seit 01.01.2008 vTI (Johann Heinrich von Thünen-Institut). 
Die Übertragung der Betriebsstrategie erfolgt am Beispiel eines typischen IFCN-Betriebes und des FADN-Vergleichsbetriebes.

Die häufigsten betrieblichen Anpassungen sind die Aufgabe oder die Ausweitung der Milchproduktion (vgl. DeEKen und Hemme, 2002, S. 14). Im Rahmen dieser Arbeit bietet es sich an, eine Wachstumsstrategie exemplarisch zu berechnen. Dabei werden zwei Wachstumsalternativen betrachtet (vgl. DEEKEN und HEMME, 2002):

- Strategie „Stall voll“: Ausdehnung der Milchproduktion im Umfang der vorhandenen Stallkapazitäten mit geringem Investitionsvolumen.

- Strategie „Anbau“: Ausbau der Milchproduktion in realistischem Umfang mit hohem Investitionsvolumen.

Eine dieser Wachstumsalternativen wird in Kapitel 7.2 in Abhängigkeit vom ausgewählten Untersuchungsbetrieb zur Simulation im TIPI-CAL-Modell bestimmt.

Die mit wenigstens einem Panel-Landwirt und einem Berater entwickelte Strategie für den IFCN-Betrieb wird über den 10-Jahreszeitraum angepasst (Land, Futteranbauverhältnis, Arbeitseinsatz, Investitionen in Stall, Maschinen, Gebäude, etc.). Dies erfolgt in einem „iterativen“ Prozess. Die entwickelte Anpassungsstrategie wird mit dem Modell berechnet. In den folgenden Panelsitzungen werden der Landwirt und der Berater mit dem Simulationsergebnis und den Eingabedaten so lange konfrontiert, bis die Plausibilität der Eingabedaten und die Modellergebnisse vom Panel bestätigt werden können. ${ }^{8}$

Die ausgewählte Strategie wird auf den FADN-Betrieb übertragen: Die Wachstumsschritte erfolgen in demselben Jahr wie beim entsprechenden IFCN-Betrieb. Die Expertenmeinungen aus den IFCN-Panelsitzungen, u. a. zur Höhe der Investition in Stallbau und Melktechnik, zur Änderung der Anbauverhältnisse, zu zusätzlich benötigten Arbeitskräften und gegebenenfalls zu zusätzlichen Leistungen durch Lohnunternehmer werden bei der Modellierung berücksichtigt. Genauere Angaben sind in Kapitel 7.3 und in der Anhangstabelle A7.2 zu finden.

Um die Fehlerrisiken zu reduzieren, die bei der detaillierten Ergänzung und Korrektur der FADN-Daten mit Hilfe von IFCN-Daten und Daten aus weiteren Quellen entstehen (vgl. IsERMEYER et al., 2003), werden die zu ergänzenden Daten mit Hilfe einer Koeffizientenschätzmethode zusätzlich auf Plausibilität überprüft und gegebenenfalls angepasst. Der Koeffizientenschätzansatz wird im folgenden Kapitel 7.2.2 erläutert.

Ausführliche Informationen zur Vorgehensweise bei der Schätzung betrieblicher Anpassungspotenziale im Rahmen des IFCN sind bei HEMME (2000) nachzulesen. 


\subsubsection{Spezifikation des Modells zur Schätzung von Koeffizienten}

Das Modell zur Schätzung von Koeffizienten kommt im Rahmen der Nutzung der FADNDaten für Berechnungen im Simulationsmodell TIPI-CAL insbesondere in den Fällen zur Anwendung, wenn gleich mehrere Koeffizienten simultan zu schätzen sind.

Beachtliche Unterschiede bei Produkt- und Faktorpreisen, Aufwandsmengen und Naturalerträgen treten schon zwischen verschiedenen Jahren und Regionen und sogar zwischen ähnlichen Betrieben auf. Hinzu kommen nicht selten systematische Verzerrungen, wenn z. B. die Produktpreise unverändert den Marktnotierungen oder die Naturalerträge und die Faktorbedarfswerte aus Beratungsunterlagen entnommen werden.

Solche Daten aus externen Quellen sind so zu korrigieren, dass sie zumindest zu den im Betrieb erhobenen Daten passen. Dieser Anspruch ist wesentlich höher als der, der den üblichen Plausibilitätskontrollen zu Grunde liegt. Es reicht nicht aus, die Daten lediglich auf ihre Größenordnung im Raster von Zweierpotenzen zu prüfen. Die Daten sollten so korrigiert werden, dass Kontrollrechnungen mit ihnen die im Betrieb erhobenen Resultate erbringen.

Ginge es nur darum, aus dem Jahreserlös eines Produktes und der Ablieferungsmenge (oder dem monetären und dem physischen Aufwand) den durchschnittlichen Preis zu errechnen, so wäre das kein Problem. Schwierig wird es aber dann, wenn

- $\quad$ nur die Summe aus den Erlösen (Kosten) mehrerer Produkte vorliegt,

- die Erträge/Aufwendungen in mehreren verschiedenen Produktlinien entstanden sind und diesen nun nachträglich zugeordnet werden sollen oder wenn

- $\quad$ ein nennenswerter Teil der Erzeugung nicht verkauft, sondern z. B. im Betrieb verfüttert wurde und dabei womöglich noch Zukauffutter substituiert hat.

In solchen Fällen sind gleich mehrere Koeffizienten (Naturalerträge/Aufwandsmengen, Preise, Prozentanteile) simultan zu schätzen.

Deshalb wird in dieser Arbeit eine bereits entwickelte Koeffizientenschätzmethode angewendet, die im Folgenden erläutert wird. ${ }^{9}$

Um die Fehlerrisiken noch weiter zu reduzieren, werden vornehmlich produktionstechnische Daten aus möglichst ähnlichen IFCN-Betrieben verwendet, um die FADN-Betriebsdaten zu ergänzen. Die Selektion möglichst gut vergleichbarer IFCN-Betriebe erfolgt mit Hilfe der Berechnung der euklidischen Distanz. 


\subsubsection{Entwicklung der Schätzung von Koeffizienten}

Ein Vorläufer des hier dargestellten Ansatzes wurde 1978 von Hinrichs (HINRICHS und НАIмвÖСK, 1980) entwickelt und als Modul eines Modells zur kurzfristigen Einkommensprojektion auf der 20. Jahrestagung der Gesellschaft für Wirtschafts- und Sozialwissenschaften des Landbaus (GeWiSoLa) in Bonn vorgestellt. Die Überbrückung von Datenlücken mit zusätzlichen Input-/Output-Koeffizienten und das automatische simultane Einpassen dieser Koeffizienten mit Korrekturfaktoren waren bereits Elemente dieses Ansatzes, ebenso die Minimierung ihrer relativen Korrekturen (Abweichung der Faktoren vom Wert 1) und die „härtespezifische“ Bewertung der Abweichungen mit „Strafkosten“. Allerdings waren die Parabeln zur Berechnung dieser Kosten nur linear approximiert, denn die Koeffizientenschätzung war damals eine vorgeschaltete Anwendung des LP-Ansatzes, mit dem anschließend auch die Projektionsrechnungen durchgeführt wurden.

Als 1993 in MS-Excel 4.0 der Solver verfügbar war, konnten die „Kosten“ als kontinuierliche exponentielle Funktion der erforderlichen Koeffizientenanpassung formuliert werden. Dieser Ansatz wurde zu mehreren Arbeiten des Institutes für Betriebswirtschaft entsprechend den individuellen Bedarfsvorstellungen der Bearbeiter herangezogen (JACOBS, 1998, s. 59-76).

Seit 1996 werden die in den Softwarepaketen (z. B. SAS) verfügbaren ökonometrischen Varianten der „Maximum Entropy Methode“ genutzt. Sie entsprechen in ihrer Wirkungsweise weitgehend dem hier beschriebenen Ansatz, dessen Anwendung sich auf pragmatische Lösungen in MS-Excel-Arbeitsblättern beschränkt.

\subsubsection{Komponenten und Wirkungszusammenhänge bei der Koeffi- zientenschätzmethode}

Im Schätzansatz werden sechs Gruppen von Informationen zusammengefügt:

(1) Grunddaten des Produktionsprogramms: Anbauflächen, Viehbestände, Arbeitskräfte (Quelle: Buchführungsergebnisse). Diese Daten sollen möglichst disaggregiert vorliegen, d. h. einzeln für alle wichtigen Produktionsprozesse. Im Bereich der Ackerwirtschaft ist dies häufig in ausreichendem Maße gegeben, weniger bei der Viehhaltung mit Ausnahme der Milchkuhhaltung.

(2) Physische und monetäre Aufwendungen und Erträge (Quelle: Buchführungsergebnisse). Gerade die physischen Daten, die für die Berechnung der gewünschten I/OKoeffizienten möglichst detailliert vorliegen sollten, sind i. d. R. gar nicht verfügbar (z. B. bei den Betriebsmitteln) oder nur für den Betrieb insgesamt (etwa beim Arbeitseinsatz). 
(3) Startwerte für die zu schätzenden I/O-Koeffizienten (aus externen Datenquellen). Anhand dieser Koeffizienten werden die Korrekturen vorgenommen. Sie sollten darum sorgfältig recherchiert und aufbereitet sein.

(4) „Härte“-Parameter, die für jeden der gewählten Startwerte spezifisch zur Geltung bringen, wie treffsicher dieser einzuschätzen ist. Ein „harter“ Koeffizient darf bei der Schätzung nur noch geringfügig verändert werden, ein „weicher“ hingegen darf in größerem Ausmaß korrigiert werden.

(5) Die Korrekturfaktoren haben zunächst alle den Wert 1,0 und werden bei Bedarf im Schätzungsalgorithmus so weit verändert, bis die mit ihnen multiplizierten Startwerte der I/O-Koeffizienten exakt zu den unter (1) und (2) genannten Betriebsparametern passen. Der Variationsbereich dieser Korrekturfaktoren liegt im Allgemeinen zwischen 0 und $\infty$, er kann aber auch enger definiert werden.

(6) Ziel ist es, die Veränderungen möglichst gering zu halten. Dies geschieht durch „Kosten“, mit denen die relativen Abweichungen von den Ausgangswerten $(1,0)$ bewertet werden. Die Funktion dieser Kosten, bezogen auf die Korrekturfaktoren, ist eine Parabel n-ten Grades, wobei der Exponent n durch die „Härte“-Parameter bestimmt wird. Alle negativen Parabelwerte (etwa bei Korrekturfaktoren $<1,0$ und ungeraden Exponenten) werden in positive umgewandelt. Am Rande des Variationsbereiches streben die „Kosten“ gegen den Wert $\infty$. Bleibt der Korrekturfaktor bei 1,0 (mithin unverändert), ist ihr Wert 0. In der Zielfunktion werden die „Kosten“ der einzelnen Korrekturen additiv (als Summanden) zusammengefasst. Der Algorithmus bestimmt dann diejenige Konstellation der Korrekturfaktoren, die die geforderte Anpassung an die Buchführungsresultate zu minimalen Gesamt-,,Kosten“ erreicht.

Ob diese Korrektur-,,Kosten“ für völlig unterschiedliche Input/Output-Koeffizienten so einfach zusammengerechnet werden dürfen, hängt davon ab, wie die Koeffizienten im Modell miteinander zusammenhängen.

- Gehören sie zu Bereichen, die funktional voneinander getrennt sind (z. B. der Kartoffelpreis und der Arbeitskraftaufwand für die Bullenmast), so sind auch ihre Korrektur„Kosten“ voneinander unabhängig und können problemlos addiert werden.

- Wirken sie multiplikativ innerhalb einer Kausalkette (z. B. dt Gerstenertrag/ha, Verkaufsanteil, Preis je dt verkaufte Gerste), so sind die Kosten ihrer Korrektur ebenfalls problemlos addierbar, da es sich ja durchweg um relative Änderungen handelt.

- Gehören die Koeffizienten aber zu Prozessniveaus, mit denen multipliziert sie einen Gesamtbetrag (Produktsumme) bilden, so kann eine einfache Addition dieser Korrektur-,,Kosten“ zu Fehleinschätzungen führen. Dann nämlich leisten die Prozesse mit hohem Niveau und/oder großen I/O-Koeffizienten einen relativ großen Korrekturbeitrag im Vergleich zu ihren Korrektur-„,Kosten“. Um auch hier eine der Aufgabe adä- 
quate Verteilung der Korrekturen auf die einzelnen Komponenten zu erreichen, sind ihre spezifischen Korrekturkosten noch mit ihrem jeweiligen Anteil am Gesamtbetrag (vor der Korrektur) zu gewichten.

\subsubsection{Berechnung und Minimierung der Korrektur-,,Kosten“6}

Im Standardfall werden für die einzuschätzenden Koeffizienten keine spezifischen Unterund Obergrenzen angegeben, so dass die Korrekturfaktoren zwischen 0 und $\infty$ liegen dürfen. In diesem Fall ist die Bewertungsfunktion relativ einfach:

Sei der Startwert eines I/O-Koeffizienten $\mathrm{A}_{0}=2,0$ und der geschätzte Wert nach $\mathrm{k}$ Verbesserungsschritten $A_{k}=1,6$ mithin der Korrekturfaktur in diesem Stadium

$$
A_{k} / A_{0}=0,8
$$

Zur Berechnung seiner „Kosten“ verwenden wir den Absolutwert seines Logarithmus. Dieser wird noch mit dem Härte-Parameter H (zum Beispiel 3) multipliziert, und den so erhaltenen Ausdruck

$$
H * \operatorname{abs}\left(\ln \left(A_{k} / A_{0}\right)\right)=3 * 0,223=0,669
$$

definieren wir als den Logarithmus der in diesem Stadium geltenden, um 1,0 erhöhten Korrektur-,,Kosten“. Diese betragen also

$$
\begin{aligned}
C_{k} & =\exp \left(H^{*} \operatorname{abs}\left(\ln \left(A_{k} / A_{0}\right)\right)\right)-1 \\
& =\exp (0,669)-1=0,953
\end{aligned}
$$

Im Falle $A_{k}=A_{0}$ hätten sie den Wert Null. Sie nehmen mit wachsender relativer Abweichung entsprechend dem Exponenten $\mathrm{H}$ zu.

\subsubsection{Einschränkungen der Nutzbarkeit der Koeffizientenschätz- methode}

Wenn der Ansatz auch eine nützliche Hilfe zur fallspezifischen Einpassung extern gewonnener Input-/Output-Koeffizienten und sogar zur Überbrückung von Datenlücken sein kann, so unterliegt seine praktische Anwendbarkeit, obwohl sie über die anderer Schätzverfahren hinausgeht, ähnlichen Einschränkungen.

Zum einen werden sie an zwei Ansatzstellen durch subjektive Annahmen und Voreinstellungen mit beeinflusst: Durch die Startwerte und die „Härte“-Parameter. Ihre Bedeutung 
ist umso größer, je komplexer das Netz von Wirkungszusammenhängen und die Anzahl der in dieses Netz einzuschätzenden Koeffizienten ist. Es ist darum nicht nur erforderlich, bei der Festlegung dieser Parameter besonders sorgfältig zu sein. Die festgelegten Annahmen und Voreinstellungen müssen auch dokumentiert werden, damit die Schätzungen nachvollziehbar sind.

Gravierender aber sind - wie bei allen anderen Schätzansätzen - die Auswirkungen von Fehlern bei der Spezifikation der Modelle und hier speziell die Nichteinbeziehung gewichtiger Komponenten, nur weil sie in den Datensätzen der Betriebe nicht erfasst sind. Soll z. B. aus der erhobenen Gesamtarbeitszeit eines Betriebes auf die Arbeitszeitbedarfswerte der einzelnen Prozesse der Pflanzen- und Tierproduktion geschlossen werden, so werden diese in aller Regel beträchtlich überschätzt. In den erfassten FADN-Arbeitszeitdaten wird häufig mehr Arbeit angegeben als effektiv angefallen ist (Hofarbeiten, etc.), so dass die Gefahr zur Überschätzung der Arbeitszeit insbesondere dann hoch ist, wenn die Arbeitszeit ausschließlich den Produktionszweigen zugeordnet wird.

\subsection{Analyse der Verknüpfung von FADN- und IFCN-Daten bei der Si- mulation von Politikänderungen am Beispiel des EU-Milch-marktes}

Um die Aussagekraft der FADN-Modellanwendungen insbesondere in Bezug auf die Übertragung betrieblicher Anpassungsstrategien und die Projektion der Betriebe in die Zukunft zu verbessern, wird der FADN-Datensatz mit Hilfe verschiedener Verfahren ergänzt und korrigiert, so dass er für Simulationsrechnungen im TIPI-CAL-Modell nutzbar gemacht werden kann.

Im Folgenden wird anhand eines Beispielbetriebs erläutert, wie der FADN-Datensatz eines Betriebs aufgrund fehlender Datentiefe - vor allem fehlende produktionstechnische Koeffizienten - modifiziert und mit Daten aus einem zuzuordnenden IFCN-Betrieb oder aus anderen Quellen angereichert werden kann, um differenzierte Simulationsrechnungen durchführen zu können. Diese Methode der Datenanreicherung ließe sich grundsätzlich auch auf viele weitere FADN-Betriebe übertragen, die den jeweiligen IFCN-Betrieben zugeordnet werden können. Dafür müsste dann allerdings die hier entwickelte Methode entsprechend programmiert werden. Nach der Erörterung der Datenmodifikation folgt die Bestimmung der Politikszenarien und Betriebsstrategien für die Projektion der Betriebe. Abschließend werden die Ergebnisse aus der Integration von FADN- und IFCN-Datensatz vorgestellt und ergänzend noch kurz mit denen aus dem IFCN-Betrieb verglichen. 


\subsubsection{Modifizierung des FADN-Datensatzes für Simulationsrechnungen im Modell TIPI-CAL}

In diesem Teilkapitel wird beschrieben, auf welche Weise der FADN-Datensatz zur Simulation im Modell TIPI-CAL nutzbar gemacht wird. Es werden nur Rechenverfahren und Variablen erläutert, bei denen Modifikationen notwendig sind. Die Anhangstabelle A7.2 liefert ergänzend einen kompletten Überblick über alle Variablen und zeigt die entsprechenden Handlungsanweisungen bei der Verknüpfung der Variablen im TIPI-CAL Modell auf.

\section{Gesamtbetriebliche Daten}

Schlachtprämie für Färsen: Die Schlachtprämie für Färsen ist im FADN-Datensatz erst ab dem Abschluss 2000/2001 erfasst. Bis zum Jahresabschluss 2000/2001 sind die Schlachtprämien für Färsen Bestandteil der Variable „Summe Rinderprämien“. Um diesen Wert getrennt zu erfassen, wird die durchschnittliche Anzahl prämienberechtigter Färsen mit der Schlachtprämie multipliziert. Aus dem FADN-Datensatz aus dem WJ 1999/2000 geht nicht hervor, wie viele Färsen geschlachtet werden. Da aber die durchschnittliche Anzahl Färsen der unterschiedlichen Altersstufen in den europäischen Buchführungsabschlüssen vorliegt, wird dieser Wert im TIPI-CAL-Modell mit dem Prozentsatz zu schlachtender Färsen aus dem IFCN-Datensatz multipliziert. Daraus resultiert die Anzahl prämienberechtigter Tiere.

Bullenprämie: Auch die Bullenprämie ist nicht separat erfasst. Sie ist ebenfalls Teil der Variable „Summe Rinderprämien“. Um die Bullenprämie herzuleiten, wurde die durchschnittliche Anzahl männlicher Rinder mit der einheitlich festgelegten Bullenprämie pro Tier multipliziert. Da im FADN in der Altersgruppe bis zu einem Jahr keine Unterscheidung zwischen männlichen und weiblichen Tieren vorliegt, wird unterstellt, dass $50 \%$ der Tiere männlich und 50 \% der Tiere weiblich geboren werden. Diese Annahme deckt sich mit den sektoralen Durchschnittswerten der vorangegangenen Jahre. Auf Basis dieser Annahme wird die durchschnittliche Anzahl prämienberechtigter Bullen ermittelt. Der resultierende Wert wird im Koeffizientenschätzmodul (siehe Kap. 7.2.2 sowie HinRICHS und НАІмвÖсK, 1980) auf Plausibilität überprüft.

Faktor „Land“: Im FADN liegen für die Untersuchungsbetriebe Hektarangaben über die gesamte landwirtschaftliche Nutzfläche, die Acker- und Grünlandfläche und die Pachtfläche (insgesamt sowie für Acker- und Grünland) vor. Die Differenz zwischen der gesamten Fläche (LF, Acker-/Grünland) und dem Pachtanteil ergibt die jeweils abgeleitete Eigentumsfläche. Der Pachtaufwand liegt im FADN nicht differenziert nach Acker- und Grünland, sondern nur für die gesamte landwirtschaftliche Nutzfläche vor. Die gesamtbetrieblichen Pachtzahlungen werden mit dem Koeffizienten „Acker- bzw. Grünlandpachtfläche (Hektar)/Gesamtpachtfläche (Hektar)“ multipliziert. Daraus resultiert, bezogen auf die 
Fläche, der durchschnittliche Pachtsatz für Acker- bzw. Grünland. Zur Kontrolle wird im Koeffizientenschätzmodul für den FADN-Betrieb mit Hilfe von bekannten regionalen Pachtsätzen der Pachtaufwand für Acker- und Grünland anteilig geschätzt. Die dabei ermittelte FADN-Gesamtpachtzahlung entspricht in etwa den IFCN-Angaben.

Faktor „Arbeit": Die Anzahl Familien- und Fremdarbeitskräfte und deren Arbeitsstunden können direkt aus dem FADN-Datensatz entnommen werden. Die Löhne für Fremdarbeitskräfte werden den im Anhangstext A6.1 beschriebenen Korrekturen unterzogen und bei Über- oder Unterschreitung des Abweichungskorridors angepasst. Die Entlohnung für die nicht entlohnten Familienarbeitskräfte erfolgt gemäß IFCN-Annahmen (vgl. Kapitel 6.3/Anhangstext A6.1). Tabelle 7.1 gibt einen Überblick über die Berechnung der Arbeitskosten.

Tabelle 7.1: Ableitung der Inputvariablen zur Berechnung der Arbeitskosten

\begin{tabular}{cll}
\hline Variable & Einheit & Ableitung aus FADN \\
\hline Familienarbeitskräfte & Anzahl & $\begin{array}{l}\text { Direkt aus dem FADN Datensatz } \\
\text { (Durchschnitt für die Vergleichsgruppe) }\end{array}$ \\
& Stunden/Jahr & $\begin{array}{l}\text { Aus dem FADN Datensatz abgeleitet: } \\
\text { Gesamtstundenzahl - Stundenzahl Lohn-AK }\end{array}$ \\
Opportunitätskosten & $€ /$ Stunde & IFCN-Annahme verwendet \\
Lohnarbeitskräfte & Stunden/Jahr & Direkt aus dem FADN Datensatz \\
Lohnkosten & $€ /$ Stunde & $\begin{array}{l}\text { Aus FADN abgeleitet: Lohnaufwand / Anzahl } \\
\text { Arbeitsstunden Lohn-AK }\end{array}$ \\
\hline
\end{tabular}

Quelle: Eigene Zusammenstellung.

Faktor „Kapital“ - Kredite: Im FADN-Datensatz liegt die Kreditsumme im WJ 1999/2000 differenziert nach kurzfristigen sowie mittel- und langfristigen Laufzeiten vor. Der FADN-Datensatz liefert keine Informationen über das genaue Jahr der Kreditaufnahme. Daher werden fiktive Kredite unterschiedlicher Laufzeiten eingesetzt, die den kurz-, mittel- und langfristigen Kreditsummen im WJ 1999/2000 für den FADNVergleichsbetrieb entsprechen.

Faktor „Kapital“ - Zinsen: Der FADN-Datensatz enthält den gesamtbetrieblichen, jährlichen Zinsaufwand. Der durchschnittliche Schuldzinssatz wird errechnet, indem der Zinsaufwand durch die Fremdkapitalsumme geteilt wird. Dabei zeigt sich, dass die Werte bei den FADN-Betrieben stark variieren. Aufgrund der hohen Varianz der FADN-Betriebe werden hier die IFCN-Zinssätze für Fremdkapital übernommen. Die Verzinsung des Eigenkapitals erfolgt ebenfalls in Anlehnung an die IFCN-Methode (vgl. Kapitel 6.3). 
Abschreibung: Die Abschreibungen im FADN sind nicht nach Maschinen und Gebäuden differenziert. Die Variable „Summe Abschreibungen“ wird entsprechend der Schätzung von Experten in 2/3 Maschinen und 1/3 Gebäude aufgeteilt. Diese Annahme deckt sich mit den sektoralen Durchschnittswerten der vorangegangenen Jahre. Die Anschaffungs- bzw. Wiederbeschaffungswerte werden so angepasst, dass im Modellergebnis die Summe der Abschreibungen mit dem FADN-Ausgangswert weitestgehend übereinstimmt. Als Annahme für den Abschreibungszeitraum wird für Maschinen 10 Jahre und für Gebäude 20 Jahre angesetzt. Diese Zeiträume decken sich mit den Angaben für den IFCN-Betrieb.

Futterzukauf für alle Betriebsaktivitäten: Zur Abbildung der Futtermittelflüsse innerhalb eines Betriebes sind Kenntnisse über den unterschiedlichen Einsatz der Futtermittel in den tierischen Produktionsprozessen erforderlich. Die europäischen Buchführungsabschlüsse geben darüber keine Auskunft. Für Marktfrüchte und für einen Teil der Futterpflanzen sind lediglich die erzeugten Mengen bekannt. Der Futtermittelzukauf (Konzentratfutter, Rau- und Saftfutter) wird im FADN in Form eines aggregierten monetären Aufwandkontos erfasst. Die Ermittlung der betrieblichen Futtermittelflüsse erweist sich vor allem deshalb als schwierig, weil ein Teil der Erzeugung nicht verkauft, sondern im Betrieb verfüttert wird und dementsprechend Zukauffutter substituiert. Daher wird der Koeffizientenschätzansatz nach HiNRICHS und НАIмвÖсK (1980) (vgl. Kapitel 7.2.2) an dieser Stelle angewendet. Als Datengrundlage für die Ermittlung verfahrensspezifischer Futterrationen sind die Informationen aus den europäischen Buchführungsdaten nicht ausreichend. Um dennoch zu verfahrensspezifischen Futterrationen für die Milchkühe und deren Nachzucht zu gelangen, werden

- Futterbedarfskalkulationen für Wiederkäuer (LANDWIRTSCHAFTSKAMMER HANNOVER UND WESER-EMS, 2004),

- Richtwerte zu Trockenmasse-, Rohprotein- und Energiegehalten von Futtermitteln (LANDWIRTSCHAFTSKAMMER HANNOVER UND WESER-EMS, 2004)

- $\quad$ und Futterrationen aus dem vergleichbaren typischen IFCN-Betrieb genutzt.

Im Koeffizientenschätzmodell unterlaufen die Futtermengen Konsistenzprüfungen in Bezug auf Energiebedarf und Energieversorgung. Die Differenz zwischen dem Energiebedarf (Erhaltungs- und Leistungsbedarf) der Rinder und der Energieversorgung aus den im Betrieb erzeugten Futtermitteln wird in Form entsprechender Mengen Konzentratfutter als auch Rau- und Saftfutter zugekauft. Die Differenz zwischen dem Energiebedarf und der Energieversorgung wird modellintern ermittelt. Die durchschnittlichen Preise für die zugekauften Futtermittel werden anhand der ZMP-Daten (ZMP, 2000 und 2001) ermittelt und stehen in Einklang mit den Futtermittelpreisangaben des typischen IFCN-Betriebes. 


\section{Pflanzenproduktion}

Im Folgenden werden die im Datensatz vorhandenen bzw. fehlenden Erträge und Preise der pflanzlichen Erzeugung aufgeführt, die aufgrund der Verknüpfung unterschiedlicher Wirtschaftsjahre und jahreszeitlicher Schwankungen einer hohen Sensibilität und Variabilität unterliegen. Da diese Werte deutlich schwanken, werden ergänzend andere Datenquellen herangezogen, sobald die FADN- und IFCN-Angaben über einen bestimmten Schwankungsbereich hinausgehen.

Erträge (Pflanzenproduktion): Bei Körnerfrüchten (Getreide, Ölsaaten, Hülsenfrüchte) sowie Zuckerrüben und Kartoffeln können die Hektarerträge unmittelbar dem FADN entnommen werden. Da die Erträge von Jahr zu Jahr sehr schwanken können, werden sie auf Plausibilität überprüft. Bei Abweichungen von mehr als $20 \%$ vom Ertrag des IFCNBetriebes bzw. vom durchschnittlichen Ertrag der amtlichen Agrarstatistik wird der verfahrensspezifische Ertrag aus dem IFCN-Betrieb bzw. wenn nicht vorhanden, der Durchschnittsertrag aus der amtlichen Agrarstatistik übernommen. ${ }^{10}$ Im Bereich Futterpflanzen (Dauergrünland, Silomais und sonstige Anbaufutterverfahren) sind im FADN Platzhalter für die Erträge der Produktionsverfahren „Silomais“, „Raues Weideland“, „temporäres Grünland“, „Futterwurzeln und Brassikazaen“, „Wiesen und Dauerweiden“ und „Getreidegrünfutter“ vorgesehen, wobei oft festzustellen ist, dass sie nicht mit Daten gefüllt sind. Alle anderen Verfahren zur Futterproduktion werden im FADN in der Kategorie „sonstige Futterpflanzen“ zusammengefasst. Beim vergleichbaren IFCN-Betrieb wird nicht zwischen Wiesen und Dauerweiden sowie Mähweiden unterschieden. Die Futterpflanzenerträge werden aus dem IFCN-Betrieb übernommen, da sie bei dem untersuchten FADNBetrieb nicht aufgezeichnet worden sind.

Outputpreise: Das TIPI-CAL-Modell benötigt Outputpreise für diejenigen pflanzlichen Produktionsverfahren, deren Erntemengen verkauft werden. Die im landwirtschaftlichen Betrieb zur betriebsinternen Verwendung erzeugten Produkte (z. B. zur betriebseigenen Futtererzeugung für die tierischen Produktionsverfahren) werden im TIPI-CAL-Modell auf Basis ihrer Produktionskosten bewertet. Dahinter steht die Annahme, dass die Produktionskosten durch den Preis gedeckt werden. Die Produktpreise für die pflanzlichen Produkte liegen in den FADN-Jahresabschlüssen nicht direkt vor. Für die wichtigsten Körnerfrüchte, für die differenzierte Erträge im FADN ausgewiesen sind, können die Outputpreise aus den in den FADN-Daten ausgewiesenen Gesamterlösen eines Verfahrens abgeleitet werden (siehe Anhangstabelle A7.2). Die Berechnung basiert auf der Annahme, dass der Konsum des Haushalts und die betriebsinterne Verwertung mit dem gleichen Preis bewertet werden wie der Verkauf des Produktes. Wenn die Outputpreise jedoch um mehr als

10

Die Erträge aus dem typischen Betrieb decken sich weitestgehend mit den Erträgen aus der amtlichen Agrarstatistik. 
20 \% von den IFCN-Outputpreisen bzw. von denen aus der Agrarstatistik (ZMP, 2000 und 2001) abweichen, werden die FADN-Preise mit den entsprechenden IFCN-Outputpreis bzw. - wenn nicht vorhanden - mit dem durchschnittlichen Erzeugerpreis aus der Agrarstatistik für das Jahr 1999/2000 (ZMP, 2000 und 2001) korrigiert. Eine Ausnahme wird lediglich bei Kartoffeln zugelassen, indem eine Schwankungsbreite von 50 \% zugelassen wird. Daher ergibt sich eine relativ große Schwankungsbreite für plausible Erzeugerpreise. Aufgrund der unterschiedlichen Vorgehensweise bei IFCN und FADN entstehen Unterschiede in den Futterkosten. Es ist aber davon auszugehen, dass die Abweichungen zwischen beiden Methoden nicht besonders groß sind.

Variable Vorleistungen: Abgesehen von den Düngemittelkosten werden im TIPI-CALModell sämtliche variable Vorleistungen lediglich durch monetäre Inputgrößen berücksichtigt. In den FADN-Jahresabschlüssen liegen für den Saatgut-, Düngemittel- und Pflanzenschutzmitteleinsatz getrennte monetäre Aufwandskonten vor. Diese Aufwandskonten sind nicht verfahrensspezifisch aufgeteilt. Die Verteilung der gesamtbetrieblichen Saatgut, Düngemittel- und Pflanzenschutzmittelkosten aus dem FADN auf die einzelnen pflanzlichen Produktionsverfahren erfolgt daher mit Hilfe der Vergleichswerte aus dem typischen IFCN-Betrieb sowie ergänzend mit Hilfe von KTBL-Planungsdaten (KURATORIUM FÜR TECHNIK UND BAUWESEN IN DER LANDWIRTSCHAFT). Die sonstigen variablen Vorleistungen im Bereich Pflanzenproduktion sind im FADN unter der Bezeichnung „Sonstige variable Kosten der pflanzlichen Produktion“ zusammengefasst. Dieses Kostenaggregat wird im TIPI-CAL-Modell auf der Kostenstelle „Sonstige variable Kosten“ übernommen und mit Hilfe der IFCN-Vergleichswerte verfahrensspezifisch zugeordnet.

\section{Produktionszweig Milcherzeugung}

Herdenmanagement: Die Ermittlung der innerbetrieblichen Wanderung einzelner Tiere von einer Altersklasse in die nächste Altersklasse bzw. von einem Produktionsverfahren in das folgende Verfahren gestaltet sich aufwendig. Als Datengrundlage bietet der FADNDatensatz Informationen über den jeweiligen Anfangs- und Endbestand sowohl als Stückzahl als auch als monetärer Wert. Ferner liefern die Buchführungsabschlüsse Auskunft über die Durchschnittsbestände, die Vieheinheiten, den Preisindex sowie über das monetäre Viehinventar und die monetäre Bestandsänderung. Diese Informationen liegen für Rindvieh der Gruppen „Rinder unter einem Jahr“, „männliche Rinder 12 bis 24 Monate“, „weibliche Rinder 12 bis 24 Monate“, „männliche Rinder älter als 24 Monate“, „Zuchtfärsen“, „Mastfärsen“, „Milchkühe“, „Schlachtkühe“, „sonstige Kühe“ und „Büffel“ vor.

Die Anzahl Kühe, Zuchtbullen, Kälber (männliche und weibliche Kälber unter einem Jahr) und Färsen (unter einem Jahr, 12 bis 24 Monate, älter als 24 Monate) werden exogen in das TIPI-CAL-Modell eingegeben. Sofern jedoch im Rahmen der Herdensimulation ein Färsendefizit oder ein Färsenüberschuss entsteht, werden diese zugekauft bzw. verkauft. Das bedeutet, dass diese betriebliche Aktivität vom TIPI-CAL-Modell automatisch simuliert wird (vgl. ISERMEYER et al., 2003). 
Im FADN-Datensatz besteht die Schwierigkeit, dass die Rinderaufzucht (bis zu einem Jahr) sowie die Kälbermast nicht nach Geschlecht unterteilt sind. Daher wird bei der Abkalbung unterstellt, dass 50 \% männliche Kälber und 50 \% weibliche Kälber geboren werden. Diese Annahme deckt sich mit den sektoralen Durchschnittswerten der vorangegangenen Jahre (vgl. ADR, 2000, Landwirtschaftskammer Schleswig-Holstein, 2001). Ferner ist zu beachten, dass die Gruppe „weibliche Rinder 12 bis 24 Monate“ nicht direkt in das TIPI-CAL-Modell in die Gruppe „Färsen 12 bis 24 Monate“ übertragen werden kann, da im FADN-Wert sowohl Milchkühe als auch Mutterkühe und Mastfärsen aufsummiert sind. Mastfärsen und Zuchtfärsen sind erst ab einem Alter von zwei Jahren im FADN getrennt erfasst. Da sich die Umtriebsrate aus dem Datensatz 1999/2000 nicht ableiten lässt, lässt sich auch der Anteil Mast- und Zuchtfärsen im gemischten Bestand (Alter geringer als zwei Jahre) nicht separat berechnen. Zur Ableitung der FADN-Herdenstruktur sind weitere produktionstechnische Informationen notwendig, die aus dem FADN-Datensatz nicht hervorgehen und daher aus dem IFCN genommen werden. Die Ermittlung der Reproduktionsleistung, die der Anzahl produzierter Jungtiere pro Kuh und Jahr entspricht (Jahresproduktion = Endbestand - Anfangsbestand + Eigenkonsum + Verkäufe - Zukäufe), ist aus dem FADN-Datensatz aus dem Jahr 1999/2000 nicht abzuleiten, da für die Verkäufe und Zukäufe keine physischen Daten, sondern nur monetäre Werte vorliegen. Diese physischen Angaben zu Viehverkäufen und -zukäufen liegen erst ab dem Jahr 2002 im FADNDatensatz vor. Es wurde versucht, eine vereinfachte Umtriebsrate ohne Zukaufs- und Verkaufszahlen zu berechnen. Dies führte jedoch zu unrealistischen Werten. Dementsprechend lässt sich die Umtriebsrate (Jahresproduktion/Durchschnittsbestand) des FADNVergleichsbetriebes im WJ 1999/2000 nur schätzen. Es wird folgende Annahme getroffen: Je höher die Milchleistung liegt, umso stärker wird selektiert. Da die Milchleistung im IFCN-Betrieb um 1,5 \% höher liegt als im FADN-Betrieb, wird für den FADN-Betrieb eine etwas niedrigere Remontierungsrate angenommen (30\%) als für den IFCN-Betrieb (35\%). Dieser Wert deckt sich mit den Informationen sektoraler Statistiken (vgl. LANDWIRTSCHAFTSKAMMER SCHLESWIG-HOLSTEIN, 2001).

Als Datengrundlage zur Ableitung der Bestandsgrößen der jeweiligen Altersstufen und Produktionsverfahren sind weitere produktionstechnische Angaben erforderlich (Erstkalbealter, Anteil lebend geborener Kälber, Zwischenkalbezeit, Anteil trocken stehender Kühe), die anhand der FADN-Daten nicht abgeleitet werden können. Daher werden die folgenden Variablen aus dem IFCN übernommen und zur Überprüfung sektorale und/oder regionale Durchschnittswerte aus externen Statistiken verwendet:

- Befruchtungsrate (ADR, 2000)

- Erstkalbealter (LANDWIRTSCHAFTSKAMMER SCHLESWIG-HOLSTEIN, 2001; ADR, 2000)

- Anteil lebend geborener Kälber (LANDWIRTSCHAFTSKAMMER Schleswig-Holstein, 2001)

- Zwischenkalbezeit (Landwirtschaftskammer Schleswig-Holstein, 2001)

- Anteil trocken stehender Kühe (Landwirtschaftskammer Schleswig-Holstein, 2001) 
- Sterberaten (Landwirtschaftskammer Schleswig-Holstein, 2001; ADR 2000)

- Zu- und Verkaufsraten (LANDWIRTSCHAFTSKAMMER SCHLESWIG-HolsteIN, 2001; ADR, 2000)

- Verkaufsgewichte (ZMP, 2003 ${ }^{11}$ )

Auf Basis dieser Informationen wird im „Milchmodul“ des TIPI-CAL-Modells die durchschnittliche Milchviehherdenstruktur für den FADN-Vergleichsbetrieb abgeleitet.

Erträge der Tierproduktion: Die Milchleistung je Kuh und Jahr lässt sich unmittelbar aus dem Jahresabschluss ableiten. Dazu muss lediglich die jährliche Milchproduktion durch den Durchschnittsbestand an Kühen dividiert werden. Für den Fett- und Eiweißgehalt werden Durchschnittswerte aus dem ZMP-Marktbericht Milch (ZMP, 1999) übernommen. Die Umrechnung auf Fett korrigierte Milch (FCM - Fat Corrected Milk) erfolgt modellintern.

Outputpreise: Der Milchpreis wird hergeleitet, indem der FADN-Gesamtumsatz mit Kuhmilch durch die Milchproduktionsmenge dividiert wird. Weil die Preise bei den FADN-Betrieben stark variieren, wird der hergeleitete Preis mit Hilfe bekannter Produktpreise (ZMP, 2003) einer Plausibilitätsprüfung unterzogen. Es wird ein Abweichungskorridor von $\pm 20 \%$ gelegt, um betriebsgruppenspezifische Unterschiede (unterschiedliche Vermarktungsbedingungen, Produktqualitäten) berücksichtigen zu können (vgl. ScHLEEF, 1999, S. 48). Falls der betriebsgruppenspezifische Preis den zulässigen Abweichungskorridor über- oder unterschreitet, wird der entsprechende Preis aus den ZMP-Markt- und Preisberichten (ZMP, 2003) eingesetzt. Auf diese Weise lässt sich verhindern, dass die Ergebnisse des FADN-Betriebes allzu sehr verzerrt werden.

Aus den monetären Gesamterlösen für Rindvieh im FADN sind keine Produktpreise für geschlachtete Bullen, Kühe, Färsen und Kälber ableitbar. Daher werden hier IFCN-Werte eingesetzt. Aufgrund der Varianzen (siehe oben) erfolgt auch hier eine Kontrolle mit gewichteten Durchschnittswerten (1998 bis 2002) aus externen Statistiken, denen wiederum eine regionale Aufteilung zwischen Nord- und Süddeutschland zugrunde liegt (ZMP, 2003). Die Lebendviehpreise für Bullen, Kühe, Kälber und Färsen werden ebenfalls aus dem IFCN übernommen und anhand der ZMP-Daten überprüft (ZMP, 2003).

Zur weiteren Aufbereitung des Datensatzes des zu verknüpfenden FADN-Betriebes wird, wie im Anhangstext A6.1 detailliert beschrieben, die Kostenallokation angewendet. Mittels unterschiedlicher Allokationsschlüssel werden ausgehend von der Gewinn-und-

11 Die Basis für die Lebend- und Schlachtgewichte von der ZMP liefert eine regionale Aufteilung zwischen Nord- und Süddeutschland. 
Verlust-Rechnung die gesamtbetrieblichen Kosten dem Betriebszweig Milch zugeteilt. Detaillierte Erläuterungen hierzu sind im IFCN-Handbuch (ISERMEYER et al., 2003) nachzulesen.

\subsubsection{Bewertung der TIPI-CAL-Modellrechnungen mit FADN-Daten- sätzen}

Die Leistungsfähigkeit der Modifikation des FADN-Datensatzes zur Nutzung im Simulationsmodell TIPI-CAL wird am Beispiel einer Änderung der EU-Milchmarktpolitik getestet. Im Folgenden werden kurz die der Politikanalyse zugrundeliegenden Szenarien vorgestellt.

Im Jahr 2003 standen vor allem folgende zwei Politikreformen im Mittelpunkt der Diskussion: der Mid-term Review (Agenda-Zwischenbewertung), der im Juni 2003 beschlossen worden ist, und die Abschaffung der Milchquotenregelung im Jahr 2008. Zum Zeitpunkt der Agenda-Beschlüsse galt die Milchquotenregelung bis zum Ende des Wirtschaftsjahres 2007/2008 als gesichert ${ }^{12}$. Daher war es naheliegend, in den Analysen einen Ausstieg frühestens in 2008 vorzusehen.

Folgende Szenarien werden analysiert:

- Referenzszenario Agenda 2000 („AG Ref.“): Milchquotenregelung, Milchpreis in 2008 -11,2 \% zu 1999, Milchprämien 2,5 €/100 kg Milch, Quotenaufstockung.

- MTR (Agenda Zwischenbewertung) Beschluss Juni 2003 (,MTR MM“): Milchquotenregelung, Milchpreis in 2008 -15 \% zu 1999, Milchprämien 3,55 €/100 kg Milch; ohne Entkopplung/Modulation ${ }^{13}$; Quotenaufstockung.

- Milchquotenausstieg (,ohne Quote“): Quotenausstieg 2008 bei gleicher Entwicklung bis 2007 und Prämien wie im Szenario „MTR MM“, Milchpreis in 2008 -22 \% zu 1999.

Die Daten wurden im Rahmen der Arbeitsgruppe „Modellgestützte Politikfolgenabschätzung“ der FAL entwickelt (vgl. KLeinhanss et al., 2001). Die Preisbedingungen im Zeitablauf von 1999 bis 2008 sind in Tabelle 7.2 für das Referenzszenario und in Tabelle 7.3 für beide Politikszenarien dargestellt.

12 Laut Health Check geht die europäische Kommission davon aus, dass die Quotenregelung zum 31.03.2015 endgültig ausläuft (EU-KOMMISSION, 2008).

13 Da die Entkopplung und Modulation bis zum Abschluss dieser Berechnungen noch nicht spezifiziert waren, sind sie hier nicht berücksichtigt worden. 
Tabelle 7.2: $\quad$ Annahmen für das Referenzszenario

\begin{tabular}{|c|c|c|c|c|c|c|c|c|c|c|c|}
\hline Jahr $\mathbf{r}$ & & 1999 & 2000 & 2001 & 2002 & 2003 & 2004 & 2005 & 2006 & 2007 & 2008 \\
\hline Interventionspreis & & & & & & & & $-5,0 \%$ & $-10,0 \%$ & $-15,0 \%$ & $-15,0 \%$ \\
\hline Erzeugerpreis zu 1999 1) & & & & & & & & $-3,6 \%$ & $-7,3 \%$ & $-11,2 \%$ & $-11,2 \%$ \\
\hline Erzeugerpreis & Index & 100 & 105 & 115 & 104 & 103 & 104 & 96 & 93 & 89 & 89 \\
\hline Quotenkaufpreis 2) & Euro/kg Quote & & 0,83 & 0,81 & 0,45 & 0,47 & 0,45 & 0,43 & 0,41 & 0,39 & 0,39 \\
\hline Milchquote $^{3)}$ & & & & & & & & $0,5 \%$ & $0,5 \%$ & $0,5 \%$ & \\
\hline Milchprämie $^{4)}$ & Euro/100kg & & & & & & & 0,833 & 1,666 & 2,499 & 2,499 \\
\hline Rindfleischpreise & & 100 & 93 & 67 & 81 & 84 & 79 & 79 & 80 & 81 & 81 \\
\hline Schlachtprämie ${ }^{5)}$ & Euro/Tier & & 34 & 66 & 100 & $-\ldots$ & $\ldots$ & $---k$ & stant - & - & - \\
\hline
\end{tabular}

1) Erzeugerpreise bis 2002/2003 wie zu beobachten, ab 2003/2004 nach FAL-Modellverbund (GAPsi-Ergebnisse).

2) Die Quotenkaufpreise (nach FAL-Modellverbund) variieren in Abhängigkeit von dem jeweiligen Bundesland; die gemachten Angaben beziehen sich auf den typischen deutschen 68-Kuh-Betrieb (Bremervörde) und sind bis zum Abschluss der Berechnungen (08/2003) aktualisiert; im Jahr 1999 k.A. aufgrund nicht handelbarer Lieferrechte.

3) Milchquotenaufstockung im Zuge der Milchmarktreform der Agenda 2000, bezogen auf die eigene Quote im Jahr 2004.

4) Milchprämie ist auf die Milchquote im Jahr 2004 bezogen.

5) Schlachtprämie für Altkühe, Schlachtfärsen und Mutterkühe.

Quelle: Eigene Berechnung in Anlehnung an IFCN Annahmen (2003).

Tabelle 7.3: Annahmen für den MTR-Beschluss (Juni 2003) und für das Szenario Milchquotenausstieg

\begin{tabular}{|c|c|c|c|c|c|c|c|c|c|c|c|}
\hline \multicolumn{2}{|l|}{ Jahr } & 1999 & 2000 & 2001 & 2002 & 2003 & 2004 & 2005 & 2006 & 2007 & 2008 \\
\hline \multicolumn{7}{|l|}{ Interventionspreis } & $-5 \%$ & $-10 \%$ & $-15 \%$ & $-20 \%$ & $-20 \%$ \\
\hline \multicolumn{7}{|c|}{ Erzeugerpreis zu 1999 1) } & $-4 \%$ & $-9 \%$ & $-13 \%$ & $-15 \%$ & $-15 \%$ \\
\hline Erzeugerpreis & Index & 100 & 105 & 115 & 104 & 104 & 96 & 91 & 87 & 85 & 85 \\
\hline \multicolumn{11}{|c|}{$\begin{array}{l}\text { Erzeugerpreis zu } 1999{ }^{1)} \text { Beispiel MQ Ausstieg } \\
\text { Erzeugerpreisindex Beispiel MQ Ausstieg }\end{array}$} & $\begin{array}{c}-22 \% \\
78\end{array}$ \\
\hline Quotenkaufpreis 2) & Euro/kg & & 0,83 & 0,81 & 0,45 & 0,47 & 0,45 & 0,43 & 0,39 & 0,31 & 0,31 \\
\hline \multicolumn{7}{|l|}{ Milchquote $^{3)}$} & & & $1,5 \%$ & & \\
\hline Milchprämie ${ }^{4)}$ & \multicolumn{6}{|c|}{ Euro/100kg } & 1,181 & 2,365 & 3,550 & 3,550 & 3,550 \\
\hline \multirow{2}{*}{$\begin{array}{l}\text { Rindfleischpreise } \\
\text { Schlachtprämie }^{5)}\end{array}$} & & \multirow[t]{2}{*}{100} & 93 & 67 & 81 & 84 & 79 & 79 & 81 & 82 & 81 \\
\hline & Euro/T & & 34 & 66 & 100 & ----- & ---- & $----k$ & stant - & -- & - \\
\hline
\end{tabular}

1) Erzeugerpreise bis 2002/03 wie zu beobachten, ab 2003/04 nach FAL-Modellverbund (GAPsi-Ergebnisse).

2) Die Quotenkaufpreise (nach FAL-Modellverbund) variieren in Abhängigkeit von dem jeweiligen Bundesland; die gemachten Angaben beziehen sich auf den typischen deutschen 68-Kuh-Betrieb (Bremervörde) und sind bis zum Abschluss der Berechnungen (08/2003) aktualisiert; im Jahr 1999 k.A. aufgrund nicht handelbarer Lieferrechte.

3) Milchquotenaufstockung: da sie bis zum Ende dieser Berechnungen noch nicht genau spezifiziert war, erfolgt die Quotenaufstockung von 1,5 \% im Jahr 2006 bezogen auf die eigene Quote im Jahr 2005; mittlerweile ist bekannt, dass die Quotenaufstockung über die Jahre 2006 bis 2008 verteilt wird (+ 0,5 \% pro Jahr); (Amtsblatt der Europäischen Union, 2003).

4) Milchprämie: hier Entkopplung/Modulation nicht mit einbezogen, da sie zum Abschluss der Berechnungen noch nicht spezifiziert war; die Milchprämie ist auf die Milchquote im Jahr 2004 bezogen; mittlerweile ist bekannt, dass künftig für die Milchprämie gilt: 2004: Quote 31.3.2004 * 1,181 c/kg (Produktion gekoppelt), 2005: Quote 31.3.2005* 2,365 c/kg (von der Produktion entkoppelt), 2006: Quote 31.3.2005 * 3,55 c/kg (von der Produktion entkoppelt); (Bauernverband Mecklenburg-Vorpommern e.V., 2004).

5) Schlachtprämie für Altkühe, Schlachtfärsen und Mutterkühe.

Quelle: Eigene Berechnung in Anlehnung an IFCN Annahmen (2003). 
Die Verknüpfung von FADN und IFCN im TIPI-CAL-Modell wird anhand eines FADNBetriebes analysiert, der eine sehr hohe Ähnlichkeit zum entsprechenden IFCN-Betrieb aufweist und auf Basis der erweiterten ${ }^{14}$ Vergleichsgruppe zum typischen 68-Kuhbetrieb in Norddeutschland gebildet worden ist. Durch den Vergleich der Ergebnisse des modifizierten FADN-Betriebes mit dem IFCN-Betrieb lässt sich die Leistungsfähigkeit des Verknüpfungsansatzes ermitteln. Werden nämlich gleiche oder sehr ähnliche Ergebnisse ermittelt, dann kann das entwickelte Verfahren der Modifikation des FADN-Datensatzes mit Hilfe von IFCN-Betriebsdaten zur Nutzung im Simulationsmodell TIPI-CAL als erfolgreich eingestuft werden.

Im Folgenden werden die FADN- und IFCN-Simulationsergebnisse in Bezug auf die zuvor beschriebenen Szenarien erläutert.

\section{Ergebnisse aus den FADN- und IFCN-Simulationsrechungen}

In Abbildung 7.2 ist die Entwicklung des betrieblichen Gewinns (in Euro pro Betrieb und Jahr) des verknüpften/modifizierten FADN-Betriebes und des IFCN-Referenzbetriebes in der Baseline mit und ohne betriebliche Anpassung dargestellt. Durch den Vergleich der jeweiligen Gewinnentwicklung können im Rahmen der Politikanalyse Aussagen über die Auswirkungen der betrieblichen Anpassungsreaktion durch betriebliches Wachstum getroffen werden. Methodisch gibt der Vergleich der Gewinnentwicklung (mit und ohne Anpassung) des FADN-Betriebes und des IFCN-Referenzbetriebes erste Auskünfte zur Bewertung der entwickelten Verknüpfungsmethode.

Die Gewinnentwicklung in der Baseline (ohne betriebliche Anpassung) ist wie folgt:

- 1999 bis 2001: Die stark ansteigenden Gewinne werden durch die steigenden Erzeugerpreise für Milch verursacht.

- 2002 bis 2004: Im Jahr 2002 sind die Gewinne durch sinkende Milchpreise niedriger als im Vorjahr und bleiben bis 2004 bei gleichbleibenden Milchpreisen auf ähnlichem Niveau.

- 2005 bis 2008: Die Milchpreissenkung aufgrund der Durchführung der Milchmarktreform der Agenda 2000 führt in den Betrieben zu Gewinneinbußen. Im Jahr 2008 steigt der Gewinn leicht an, da bei gleichbleibender Milchleistungssteigerung pro Jahr ${ }^{15}$ die Milchpreise nicht weiter sinken und die Prämien auf gleicher Höhe wie im Jahr 2007 bleiben. 
Abbildung 7.2: $\quad$ Entwicklung des betrieblichen Gewinns (in Euro) in der Baseline mit und ohne betriebliche Anpassung beim FADN-Betrieb im Vergleich zum typischen IFCN-Betrieb

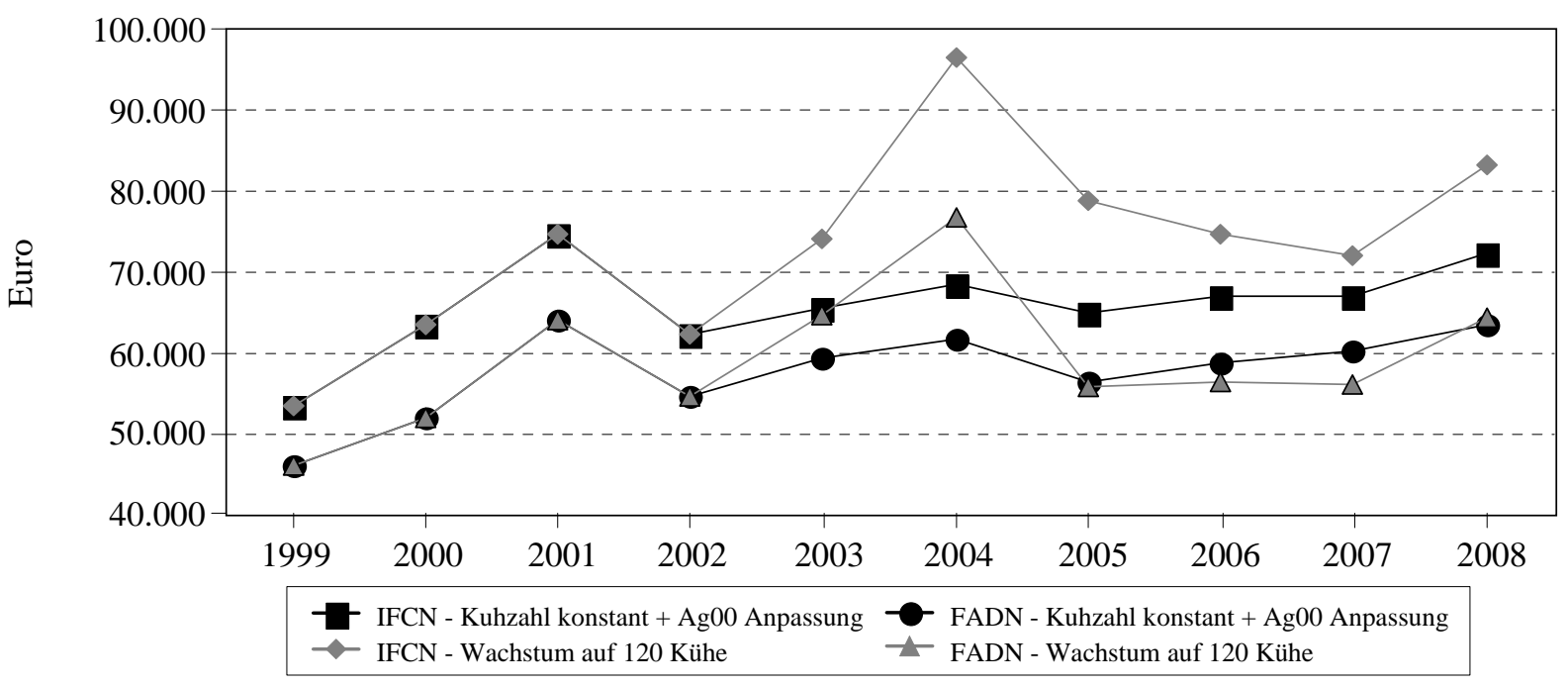

Quelle: Eigene Berechnung und Darstellung mit IFCN und FADN (2004).

Unterschiede im Gewinnniveau bei FADN und IFCN sind bedingt durch etwas höhere Erlöse (u. a. Ackerbauerlöse, Nebenerlöse Milch und Zinserlöse) bei IFCN sowie geringfügige Unterschiede bei den Anteilen betriebseigener Faktoren (u. a. Milchquote). Trotz etwas unterschiedlichem Gewinnniveau sind die Gewinnverläufe bei beiden Betrieben recht ähnlich. Deutlich wird, dass erwartungsgemäß bei beiden Betrieben eine Vergleichbarkeit in der Betroffenheit auf Politikeinflüsse gegeben ist.

Als eine betriebliche Anpassung ist die Ausdehnung der Milchproduktion über einen Stallanbau (um 52 Kühe beim IFCN-Betrieb) gewählt worden (vgl. Kapitel 7.2), die im Rahmen eines FAL-Projektes (DEEKEN und HEMME, 2002) in Zusammenarbeit mit der Landwirtschaftskammer Schleswig-Holstein ${ }^{16}$ entwickelt worden ist. Die Erweiterung der Milchproduktion spiegelt eine realitätsnahe Anpassungsstrategie für diesen Betriebstyp wider. Das betriebliche Wachstum erfolgt in zwei Schritten. Im Jahr 2003 haben die Betriebe ihre Kuhzahl von 68 auf 80 und in 2004 nochmals auf 120 Kühe erhöht. Es wird im Rahmen der durchgeführten Wachstumsschritte annahmegemäß keine Verschlechterung der Produktionstechnik (z. B. Remontierungsrate, Sterberate, Tierarztkosten pro Kuh) unterstellt (vgl. KLEINHANSS et al., 2001). 
Tabelle 7.4: Wichtige Betriebskennzahlen in der Ausgangssituation und nach einem Wachstumsschritt auf 120 Kühe

\begin{tabular}{|c|c|c|}
\hline & $\begin{array}{l}\text { Ist-Situation } \\
68 \text { Kühe }\end{array}$ & $\begin{array}{l}\text { Stallanbau } \\
120 \text { Kühe }\end{array}$ \\
\hline Fläche & $\begin{array}{l}42 \text { ha Eigen } \\
48 \text { ha Pacht }\end{array}$ & $\begin{array}{l}42 \text { ha Eigen } \\
60 \text { ha Pacht }\end{array}$ \\
\hline Anbauverhältnis & $\begin{array}{l}20 \text { ha Mais } \\
36 \text { ha Grünland } \\
29 \text { ha Getreide } \\
5 \text { ha Stilllegung }\end{array}$ & $\begin{array}{l}21 \text { ha Mais } \\
48 \text { ha Grünland } \\
28 \text { ha Getreide } \\
5 \text { ha Stilllegung }\end{array}$ \\
\hline \multicolumn{3}{|l|}{ Arbeitskräfte } \\
\hline Fam-AK & $\begin{array}{c}2 \mathrm{AK} \\
4.800 \mathrm{~h}\end{array}$ & $\begin{array}{c}2 \mathrm{AK} \\
4.800 \mathrm{~h}\end{array}$ \\
\hline Aushilfen & $400 \mathrm{~h}$ & $960 \mathrm{~h}$ \\
\hline $\begin{array}{l}\text { Investitionen } \\
\text { Stall }\end{array}$ & $\begin{array}{c}1981 \text { Boxenlaufstall } \\
1990 \text { Jungviehstall }\end{array}$ & $\begin{array}{c}\text { Anbau Kuhstall } \\
\text { Preis: } 86.920 € \\
35.790 € \text { in Futtertechnik }\end{array}$ \\
\hline Gülle & 1986 Güllesilo & - \\
\hline Melktechnik & $\begin{array}{c}1993 \text { Rep. Melkanlage } \\
\text { Preis: } 10.226 €\end{array}$ & $\begin{array}{c}\text { Anbau auf } 2 \text { × } 8 \\
\text { Melkstand }(61.355 €)\end{array}$ \\
\hline Maschinen & $\ldots$ & \\
\hline
\end{tabular}

\section{Lohn unternehmer}

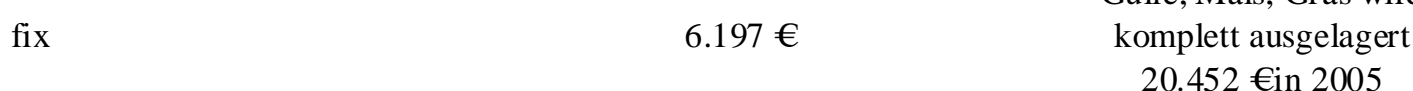

\begin{tabular}{lcc}
\hline Gerste und Roggen & $142 € /$ ha & $142 € /$ ha \\
\hline Stroh & $82 € /$ ha & $82 € /$ ha \\
\hline Grassilage & $179 € /$ ha & $179 € / \mathrm{ha}$ \\
\hline Maissilage & $164 € /$ ha & $164 € /$ ha \\
\hline insgesamt & $\mathbf{1 8 . 2 0 6} €$ & $\mathbf{3 8 . 0 9 1} €$ \\
\hline Sonstiges & 35 Bullen & keine \\
Bullen & $100 \%$ der & $100 \%$ der \\
\hline Färsenaufzucht & Kuhkälber & Kuhkälber \\
\hline Marktfrucht & 29 ha & 28 ha \\
\hline Fixkosten & $51.640 €$ & $71.581 €$ \\
\hline
\end{tabular}

Quelle: E. Deeken, T. Hemme (2002); Experteneinschätzung J. Thomsen, LWK Schleswig-Holstein. 
Eine Übertragung der Strategie „Ausnutzung der vorhandenen Stallkapazitäten“ scheidet für den FADN-Betrieb aus, da Informationen zu vergangenen Investitionen im FADNDatensatz nicht vorliegen und vorhandene Stallkapazitäten daher schwer abzuschätzen sind. Der betriebliche Wachstumsschritt auf 120 Kühe tangiert im Wesentlichen folgende Bereiche: Land, Futteranbauverhältnis, Arbeitseinsatz, Investitionen in Stall, Maschinen, Gebäude. Bei der Übertragung der Wachstumsstrategie auf den FADN-Betrieb werden die in Tabelle 7.4 erwähnten Größenordnungen den FADN-Betriebsdaten entsprechend angepasst.

Wie Abbildung 7.2 zeigt, führt die Ausweitung der Milchproduktion bei beiden Betrieben zu einer Steigerung der Gewinne in 2003 und 2004. Die Milchmarktreform der Agenda 2000 und die höheren Verbindlichkeiten führen in 2005 jedoch zu deutlichen Gewinneinbußen. Erst im Jahr 2008 steigt der Gewinn, ähnlich wie in der Baseline ohne betriebliches Wachstum, wieder leicht an. In 2008 liegt der Gewinn bei dem FADN-Betrieb in etwa auf dem Niveau des Gewinns ohne betriebliches Wachstum. Beim IFCN-Betrieb liegt der betriebliche Gewinn in 2008 etwas höher als im Szenario ohne Wachstum. Diese Unterschiede sind u. a. dadurch bedingt, dass der FADN-Betrieb eine geringere durchschnittliche Liquidität und weniger Eigenkapital aufweist als der IFCN-Betrieb und sich die Finanzierung der Investition stärker im betrieblichen Gewinn niederschlägt.

Auch bei wachsendem Kuhbestand liegen erwartungsgemäß ähnliche Gewinnentwicklungen beim FADN-Betrieb und beim IFCN-Referenzbetrieb vor (Abbildung 7.2). Der ansteigende Gewinn deutet darauf hin, dass die positiven Effekte des Wachstumsschritts nach dem Jahr 2008 deutlicher werden. Dennoch erweist sich ein moderateres Wachstum für den 68Kuhbetrieb möglicherweise als günstiger. Bei einer Ausweitung der Milchproduktion um 76 \% (+ 52 Kühe) werden verstärkt Aushilfskräfte sowie Lohnunternehmer eingesetzt, so dass die zusätzlichen wachstumsbedingten Erlöse größtenteils durch die zusätzlichen Kosten aufgezehrt werden (KLEINHANSS et al., 2001). Als sinnvoller gilt in der Regel für diesen Betriebstyp die Ausdehnung der Milchproduktion im Rahmen der zur Verfügung stehenden Stallkapazitäten (+ 18 \% auf 80 Kühe), wobei annahmegemäß keine Investitionen in Stallungen und Melkanlage nötig sind. Da aber die Informationen zu vergangenen Investitionen im FADN-Datensatz nicht vorliegen und vorhandene Stallkapazitäten daher schwer abzuschätzen sind, wurde der größere Wachstumsschritt ausgewählt. Eine Lösungsmöglichkeit besteht darin, die mit Beratern ermittelten freien Stallkapazitäten für den IFCN-Betrieb auf den FADNBetrieb zu übertragen.

\section{Effekte des MTR-Beschlusses und eines fiktiven Milchquotenausstiegs (ohne Anpassung)}

Anhand des IFCN-Milchviehbetriebes und seines FADN-Vergleichbetriebes werden die Einkommenseffekte des MTR-Beschlusses (aus dem Jahr 2003) im Vergleich zur Agenda 2000 und einem Milchquotenausstieg analysiert. Im ersten Schritt dieser Analyse werden potenzielle Anpassungsreaktionen der Betriebe nicht berücksichtigt. 
Abbildung 7.3 stellt die Entwicklung des betrieblichen Gewinns im Betriebszweig Milcherzeugung bei dem Kommissionsbeschluss und einem fiktiven Quotenausstieg im Jahr 2008 im Vergleich zur Fortführung der Agenda 2000 in der Baseline dar.

Abbildung 7.3: $\quad$ Entwicklung des Gewinns im Betriebszweig Milch (Euro pro $100 \mathrm{~kg}$ Milch) in den Szenarien MTR-Beschluss und Milchquotenausstieg beim FADN-Betrieb im Vergleich zum IFCN-Betrieb ohne Anpassung

\section{IFCN Betrieb}
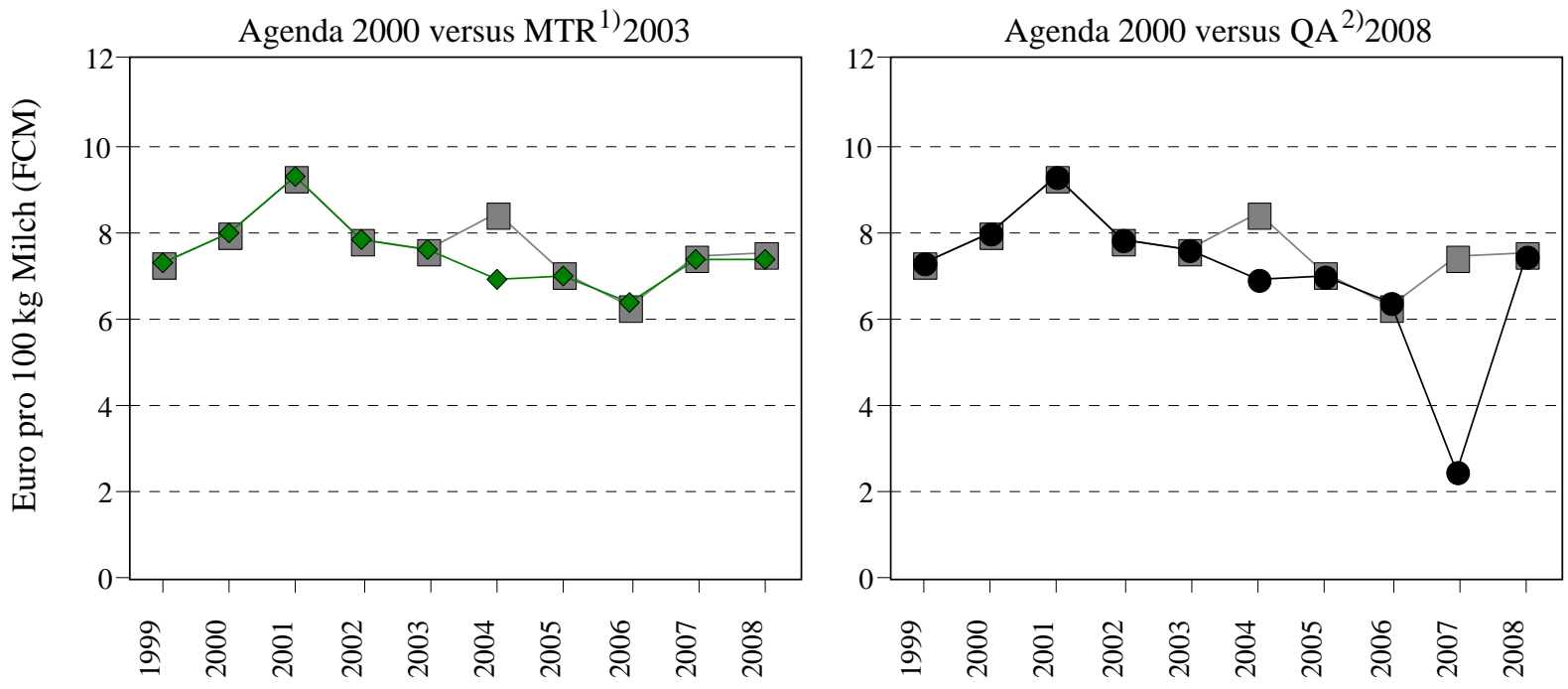

FADN Vergleichsbetrieb
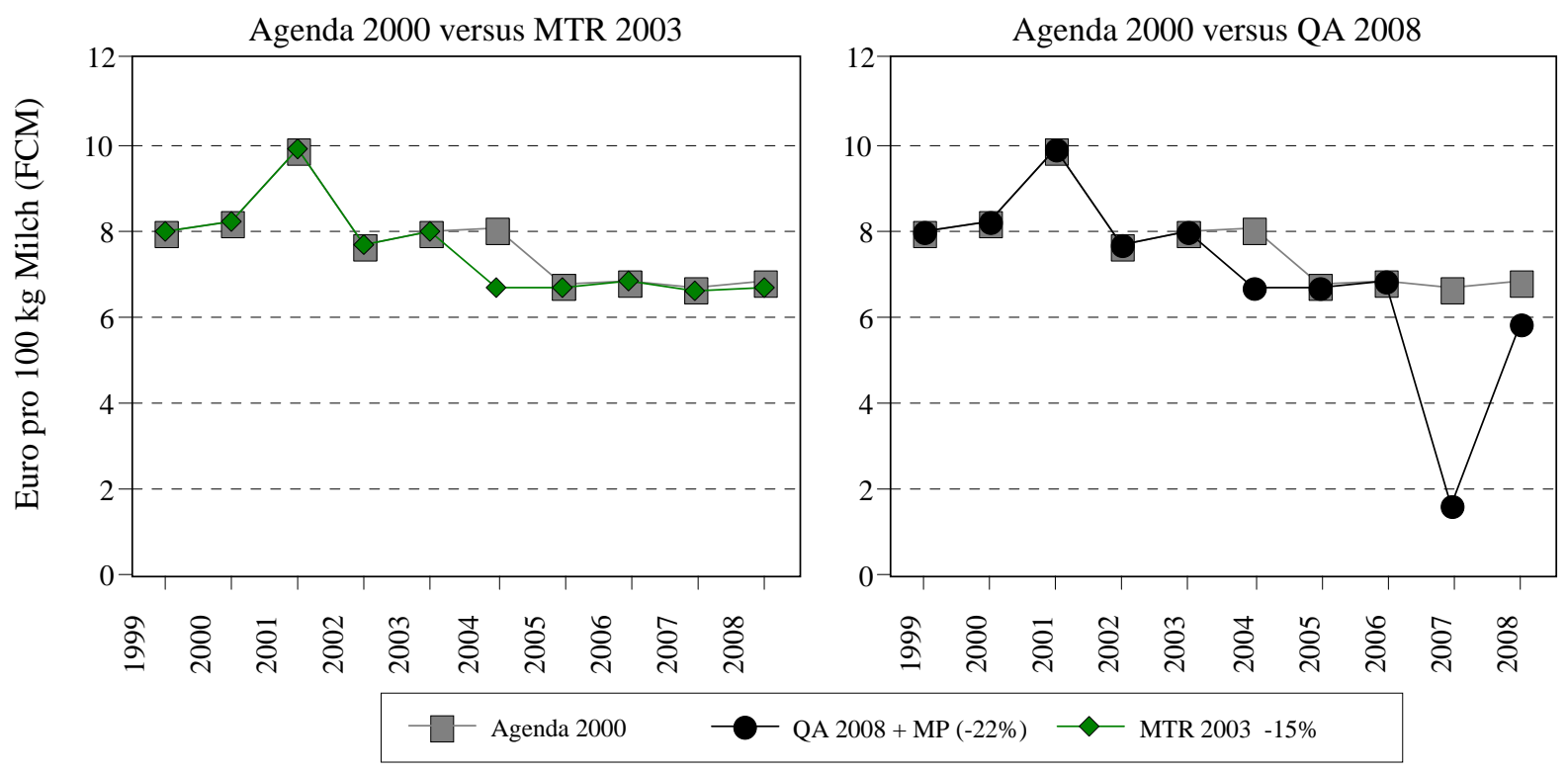

1) MTR = Mid-term-review.

2) $\mathrm{QA}=$ Milchquotenausstieg.

Quelle: Eigene Berechnung und Darstellung. 
Die Betroffenheit der Betriebe wird durch die Veränderung des Gewinns in Euro je $100 \mathrm{~kg}$ Milch deutlich. Wie Abbildung 7.3 zeigt, führt die vorgezogene Reform des Milchmarktes in 2004 bei beiden Betrieben aufgrund der vorgezogenen Milchpreissenkung (-3€/100 kg Milch) zu Einkommenseinbußen (-17,1 \% vs. -18,5 \% beim IFCN-Betrieb), die durch steigende Direktzahlungen (1,83 €/100 kg Milch) nicht kompensiert werden können. Bezogen auf das Jahr 2008 bewirkt der MTR-Beschluss im Vergleich zur Agenda 2000 jedoch keine signifikanten Einkommensverluste, da die Milchpreissenkung von $1 € / 100 \mathrm{~kg}$ Milch durch zusätzliche Direktzahlungen kompensiert wird.

Im Szenario Milchquotenausstieg zeigen sich beim IFCN-Betrieb und beim FADNVergleichsbetrieb bis 2007 im Vergleich zum Kommissionsbeschluss keine größeren Einkommenseffekte. Bei einem Milchquotenausstieg im Jahr 2008 ergibt sich im Jahr 2007 durch einmalige Abschreibung der gekauften Quote ein einmaliger Einkommenseffekt von 2 bis $3 € / 100 \mathrm{~kg}$ Milch. Dieser negative Einkommenseffekt fällt beim FADN-Betrieb höher aus aufgrund eines höheren Anteils gekaufter Quote. In 2008 steigt das Einkommen bei allen Betrieben wieder an. Der IFCN-Referenzbetrieb besitzt einen größeren Anteil an Pachtquote. Annahmegemäß werden keine Schadensersatzzahlungen aufgrund wegfallender Quotenpachtzahlungen getroffen. Daher sind bei einem Quotenausstieg für den FADNBetrieb in 2008 stärkere negative Einkommenseffekte in der Höhe von -0,98 €/100 kg Milch zu verzeichnen im Vergleich zu -0,12 €/100 kg Milch beim IFCN-Betrieb.

\section{Effekte des MTR-Beschlusses und eines fiktiven Milchquotenausstiegs (mit Anpassung)}

Betriebsanpassungen ohne Politikänderungen, die für realistische Projektionen beachtet werden sollten, wurden bereits in Abbildung 7.2 aufgezeigt. Darüber hinaus wird im Folgenden auf betriebliche Anpassungsreaktionen eingegangen, die häufig aufgrund von Politikänderungen induziert werden. Die Einkommenseffekte der politikbedingten betrieblichen Anpassungen sind in Abbildung 7.4 aufgeführt.

In Abbildung 7.4 ist der Verlauf des betrieblichen Gewinns (1999 bis 2008), bezogen auf 100 kg Milch bei einer Ausdehnung der Milchproduktion in 2003 und 2004, dargestellt. In diesem Fall wird angenommen, dass Wachstum in der Baseline nicht realisiert wird. Als Referenz dient die Baseline bei konstanter Kuhzahl. In 2003 werden die steigenden Milcherlöse durch Wachstum bei beiden Betrieben von 68 auf 80 Kühe deutlich. In 2004 überwiegt beim Kommissionsbeschluss und Milchquotenausstiegsszenario der Effekt der vorgezogenen Milchpreissenkung, der durch Größenwachstum und Prämien nicht kompensiert wird und bei beiden Betrieben zu Gewinneinbußen führt. Ab 2005 gewinnt die betriebliche Anpassung an Vorteilhaftigkeit. Die Gewinneinbußen werden durch Größenwachstum kompensiert. Abbildung 7.4 verdeutlicht, dass auch der FADN-Betrieb die Einkommenseinbußen, die durch den MTR-Beschluss oder durch einen Ausstieg aus der Milchquotenregelung im Jahr 2008 entstehen, durch betriebliche Anpassung (Wachstum auf 120 Kühe) kompensieren kann. Durch betriebliche Anpassung in den Jahren 2003 und 
2004 hat er das Potenzial, einen höheren Gewinn als in der Baseline zu erzielen $(+1$ bis $1,5 €$ vs. 1 bis $1,8 €$ beim IFCN-Betrieb).

Allerdings ist zu bedenken, dass unter Agenda-Bedingungen die Durchführbarkeit des betrieblichen Wachstums nur dann gegeben ist, wenn Milchquote zugekauft werden kann. Zudem wurde in den Berechnungen nicht berücksichtigt, dass es bei großen Wachstumsschritten zu Einschnitten in der Remontierungsrate, Sterberate, Milchqualität etc. kommen kann. Da noch nicht bekannt war, wie die steuerlichen Optionen der Betriebe bei einem Milchquotenausstieg sein werden, wurde im Rahmen der IFCN-Analysen zu den Auswirkungen eines Milchquotenausstiegs (KLEINHANSS et al., 2001) die Annahme getroffen, dass den Landwirten im Jahr vor dem Quotenausstieg eine Sonderabschreibung für die restlich vorhandene Milchquote gewährt wird. Diese Annahme wird hier übernommen und führt insbesondere bei betrieblichem Wachstum aufgrund der noch kurzfristig zugekauften Milchquote zu starken Einkommenseinbußen im Jahr 2007. Der Kurvenverlauf im Szenario Milchquotenausstieg (Abbildung 7.4) wirkt dadurch irritierend, für den Vergleich der Ergebnisse aus dem FADN und IFCN ist das jedoch unerheblich.

Die folgende Abbildung 7.4 verdeutlicht, dass der IFCN-Betrieb und der FADNVergleichsbetrieb durch Politikänderungen erwartungsgemäß ähnliche Betroffenheiten und Anpassungspotenziale aufweisen. Beide Betriebe sind in der Lage, die politikbedingten Einkommenseinbußen durch betriebliche Anpassung in den Jahren 2003 und $2004 \mathrm{zu}$ kompensieren.

Diese Ergebnisse könnten ein Hinweis dafür sein, dass langfristig eine Milchprämie in der derzeitigen Größenordnung nicht notwendig ist. Bei den hier durchgeführten Berechnungen zu betrieblichem Anpassungsverhalten sind allerdings betriebliches Risiko (insbesondere bei tierischen Produktionsverfahren) und Faktorknappheit (insbesondere Land und qualifizierte Arbeit) nicht berücksichtigt worden. Auch diese Kriterien können für die langfristige Ausgestaltung der Agrarpolitik von Bedeutung sein. Hier besteht weiterer Untersuchungsbedarf.

Abschließend lässt sich festhalten, dass mit dem verknüpften FADN-Betrieb plausible und repräsentative Simulationsergebnisse zu erzielen sind, die vergleichbar sind mit den Ergebnissen für den typischen IFCN-Betrieb. Die FADN-Daten konnten mit Hilfe der Anreicherung aus dem IFCN und anderen Datenquellen für Berechnungen im Modell TIPI-CAL nutzbar gemacht werden. Grundsätzlich lässt sich das entwickelte Verfahren der Verknüpfung von FADN- und IFCN-Daten auch auf viele andere FADN-Betriebe übertragen. Für eine massenhafte Anwendung wäre jedoch noch ein entsprechendes Computerprogramm zu erstellen. 
Abbildung 7.4: $\quad$ Entwicklung des Gewinns im Betriebszweig Milch (Euro pro $100 \mathrm{~kg}$ Milch) nach betrieblicher Anpassung beim FADN-Betrieb im Vergleich zum IFCN-Betrieb

Typischer Betrieb
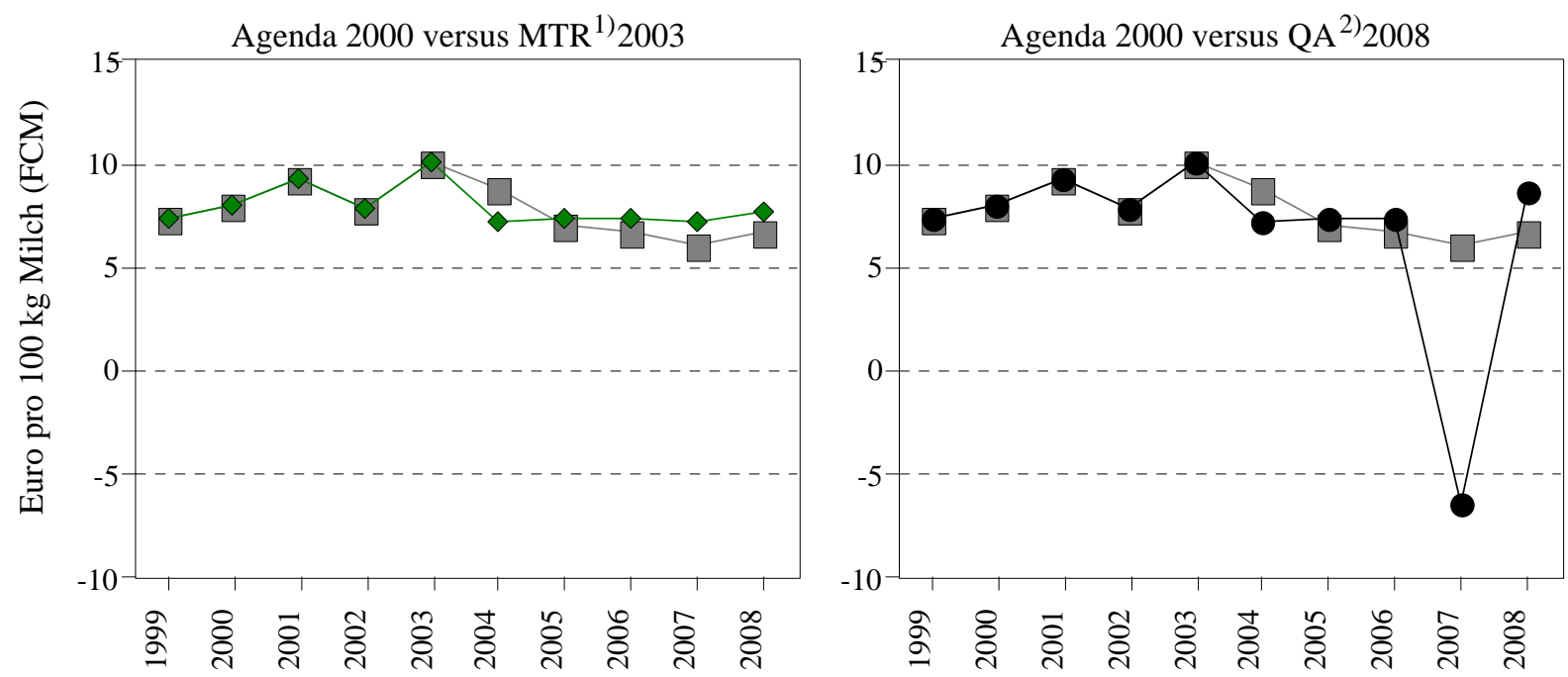

FADN-Vergleichsbetrieb
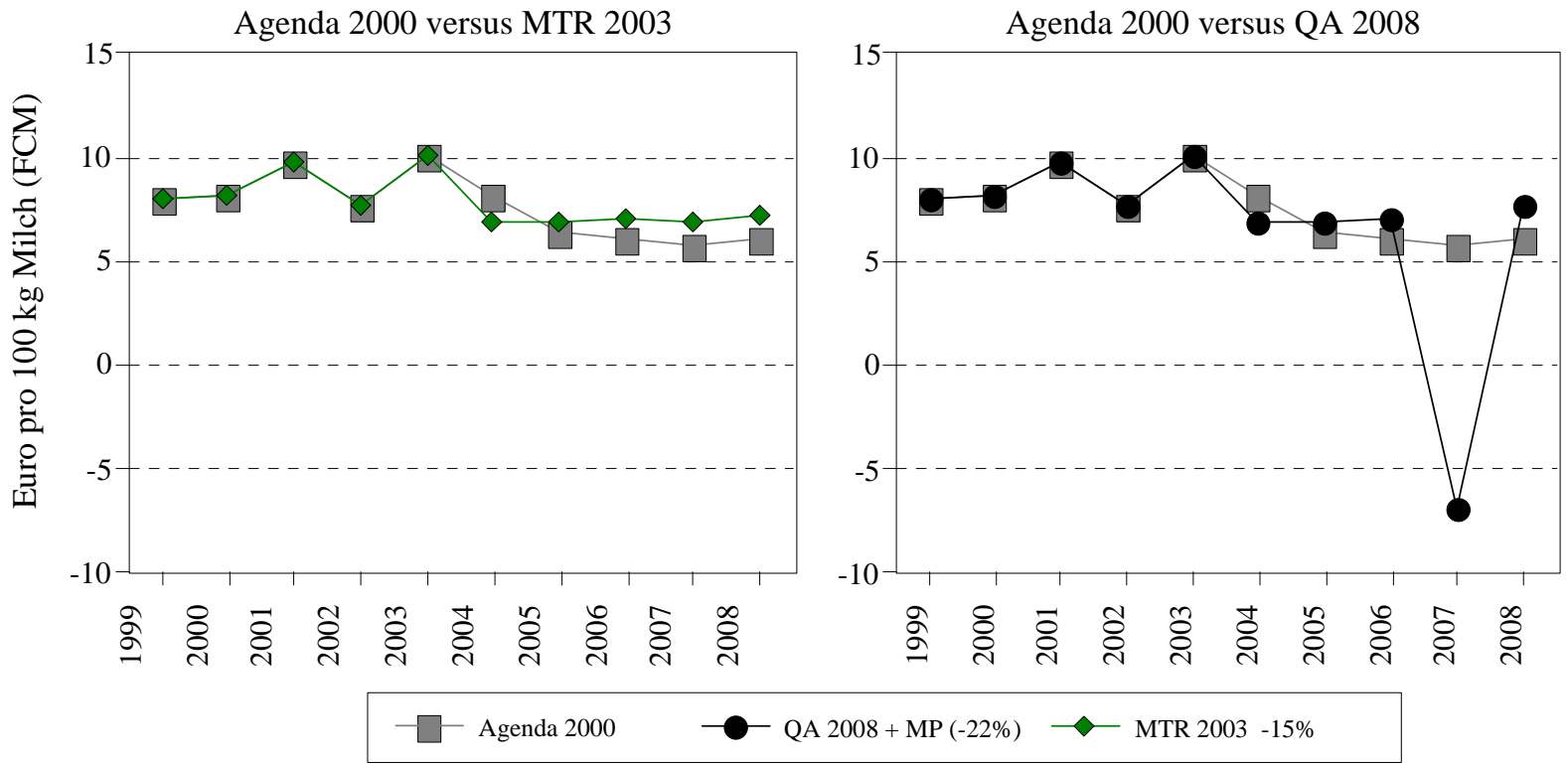

1) MTR = Mid-term-review.

2) $\mathrm{QA}=$ Milchquotenausstieg.

Quelle: Eigene Berechnung und Darstellung. 


\subsubsection{Diskussion und Schlussfolgerungen}

Das Ziel des zweiten Verknüpfungsansatzes ist die Nutzbarmachung von FADNBetriebsdaten zur Simulation im sehr detaillierten und differenzierten Modell TIPI-CAL. Hintergrund ist die Tatsache, dass die FADN-Betriebe eine größere Anzahl von Betrieben repräsentieren als der einzelne IFCN-Betrieb. Nach einer Übertragung der in dieser Arbeit entwickelten Verknüpfungen und Modifizierungen in ein Computerprogramm könnte auch eine Vielzahl von FADN-Betrieben mit Hilfe des Simulationsmodells TIPI-CAL analysiert werden.

Die FADN-Daten werden im Modell TIPI-CAL aus Konsistenzgründen und hinsichtlich des Ziels dieser Arbeit vornehmlich mit IFCN-Betriebsdaten verdichtet, um sie für Berechnungen im TIPI-CAL-Modell kompatibel zu machen. Zur Kontrolle und Ergänzung werden externe Daten aus der allgemeinen Agrarstatistik verwendet. Um die Fehlerrisiken bei der Verwendung von Daten aus unterschiedlichen Quellen zu reduzieren und in besonders schwierigen Fällen ${ }^{17}$ Näherungswerte für die Datenlücken im FADN-Datensatz zu ermitteln, wird der Koeffizientenschätzansatz nach HiNRICHS und HAIMBÖCK (1980) ergänzend angewendet.

\section{FADN-Folgenabschätzungen im Modell TIPI-CAL}

Um die Leistungsfähigkeit des Verknüpfungsansatzes beurteilen zu können, werden die Simulationsergebnisse des FADN-Betriebes mit denen des entsprechenden IFCNBetriebes verglichen.

Es konnte gezeigt werden, dass die Entwicklung des betrieblichen Gewinns (1999 bis 2008) unter Agenda-Bedingungen als auch im Hinblick auf den MTR-Beschluss und einen Milchquotenausstieg plausibel ist.

\section{Betriebliche Anpassungsreaktionen}

Auch bei Berücksichtigung betrieblichen Wachstums zeigen sich bei beiden Betrieben ähnliche Gewinnentwicklungen. Dass der FADN-Betrieb niedrigere Kosten verzeichnet als der IFCN-Betrieb liegt u. a. an der unterschiedlichen Bewertung der Nebenerlöse und der kalkulatorischen Faktorkosten. Die unterschiedliche Höhe der Nebenerlöse ist u. a. auf Datenprobleme im FADN-Datensatz zurückzuführen. Das Problem der Erfassung und Bewertung der kalkulatorischen Kosten, insbesondere der Festsetzung einer realistischen Arbeitsentlohnung, konnte im Rahmen dieser Arbeit nicht gelöst werden. Im Hinblick auf eine verbesserte Erfassung und Bewertung der kalkulatorischen Faktorkosten besteht wei-

17 Besonders schwierige Fälle liegen vor, wenn mehrere Koeffizienten simultan beteiligt und demzufolge auch simultan zu schätzen sind. 
terer Forschungsbedarf. Der Fehler, der bereits beim Betriebsvergleich im Startjahr der Simulation festgestellt worden ist, pflanzt sich über den gesamten Simulationszeitraum fort und verursacht daher auch weniger sichere Betriebsergebnisse am Ende der Simulationsperiode. Zudem erfolgte die Übertragung der typischen Anpassungsstrategie auf den FADN-Betrieb ohne Rückkopplung mit dem Expertenpanel.

Auf Basis dieser Erfahrungen wird als Verbesserungsvorschlag empfohlen, den FADN Betrieb mit Experten-Know-how aus der Region „abzurunden“ (insbesondere bezogen auf die Erfassung der Nebenerlöse und des Arbeitsinputs) und die resultierenden Simulationsergebnisse im iterativen Prozess mit den zuständigen Beratern aus dem IFCN zu diskutieren und zu kontrollieren.

Erwartungsgemäß zeigen die FADN- und IFCN-Betriebe mit und ohne Berücksichtigung betrieblicher Anpassungsreaktionen ähnliche Ergebnisse. Von daher böte es sich an, die entwickelte Verknüpfung in ein Computerprogramm zu überführen, um ohne viel Aufwand eine größere Zahl von FADN-Betrieben mit Hilfe differenzierter Simulationsmodelle analysieren zu können (auch detaillierte Kostenanalysen wären denkbar). Damit wären auch Aussagen über die Varianz der Ergebnisse und die individuelle Betroffenheit der Betriebe möglich. Durch die Verknüpfung der Daten und Informationen aus den beiden Konzepten ist es darüber hinaus möglich, bei der Modellierung realitätsnahe Anpassungsreaktionen bei einer großen Zahl von Betrieben zu berücksichtigen. Das ist vor allem deshalb wichtig, weil Politikfolgenabschätzungen ohne Berücksichtigung realitätsnaher, betrieblicher Anpassungsreaktionen zu Fehleinschätzungen führen können. Einschränkend ist allerdings anzumerken, dass für weniger komplexe Fragestellungen eine einfache komparativ-statische Analyse wahrscheinlich ausreichend ist. Es wäre im Einzelfall zu prüfen, inwiefern komparativ-statische Analysen im politischen Entscheidungsprozess für bestimmte Fragestellungen ausreichen und wann differenziertere Simulationsmodelle erforderlich sind.

Mit Hilfe der Simulationsrechnungen im TIPI-CAL-Modell lässt sich insbesondere prüfen, wie sich ein Betrieb in Abhängigkeit von den politischen Rahmenbedingungen im zeitlichen Ablauf entwickeln kann. Es können dynamische Anpassungsreaktionen im Zeitverlauf berücksichtigt und die Liquidität abgeschätzt werden. Die komparativ-statische Berechnungsmethode ist beispielsweise gut geeignet, um die Wirkung einer Politikänderung auf den betrieblichen Gewinn, verglichen mit der Referenzsituation im Jahr 2008, aufzuzeigen. Eine interessante Forschungsarbeit wäre zu untersuchen, welcher konkrete Fortschritt bei der Politikberatung mit dynamischen im Vergleich zu komparativ-statischen Analysen erzielt werden kann. Zu überlegen wäre, die politischen Entscheidungsträger mit Ergebnissen aus dynamischen und komparativ statischen Analysen zu konfrontieren, um zu überprüfen, welche Ergebnisse in der Politik besser verwertbar sind. 
Die vorgestellte Methodik verdeutlicht, dass sich mittels Verknüpfung die Vorzüge beider Datensätze kombinieren lassen. IFCN liefert im Wesentlichen für FADN physische Parameter und ermöglicht die Aktualisierung der in zeitlicher Verzögerung erscheinenden FADN-Daten und die Projektion dieser Daten in die Zukunft. Dies erfolgt durch Indizierung der FADN-Betriebe mit Hilfe der Preis- und Mengendaten aus dem IFCN bis in das aktuelle Untersuchungsjahr bzw. für den gesamten Simulationszeitraum.

Es konnte aufgezeigt werden, dass beide Datensätze großes Potenzial haben und für die Politikfolgenabschätzung wertvoll sind. Es ist sinnvoll, beide Datensätze auch weiterhin parallel in der Politikfolgenanalyse zu nutzen. Bei beiden Datengrundlagen gibt es Verbesserungsmöglichkeiten. Die Stärke von IFCN liegt in der Erhebung und Modellierung von realitätsnahem Anpassungsverhalten. Die Stärke des FADN-Datensatzes liegt in der Möglichkeit, für eine große Anzahl von Betrieben Analysen vorzunehmen und damit auch Aussagen zur Varianz der Ergebnisse geben zu können. Um allerdings realitätsnahe Anpassungsreaktionen bei den Analysen berücksichtigen zu können, sind zusätzliche Erhebungen notwendig. Wie diese europaweit zu organisieren wären, sollte in weiteren Vorhaben geklärt werden. 


\section{Zusammenfassung}

Eine wichtige Aufgabe der wissenschaftlichen Politikberatung besteht darin, mit Hilfe von Modellanalysen quantitativ abzuschätzen, wie sich agrarpolitische Reformen und sich ändernde Rahmenbedingungen auf landwirtschaftliche Betriebe auswirken und in welchem Umfang sich die Betriebe anpassen können. Bisher durchgeführte Analysen, die auf der einzelbetrieblichen Ebene ansetzen, basieren im Wesentlichen auf Buchführungsergebnissen des Informationsnetzes landwirtschaftlicher Buchführungen (INLB, englische Bezeichnung: FADN) oder auf Daten von typischen Modellbetrieben aus dem International Farm Comparison Network (IFCN). Für kurzfristige Abschätzungen ohne nennenswerte Mengenänderungen bieten die FADN-Daten gute Voraussetzungen. Für differenziertere Analysen unter Berücksichtigung betrieblicher Anpassungen fehlt den FADN-Daten jedoch die erforderliche Datentiefe, insbesondere im Hinblick auf das Mengengerüst und die verfahrensspezifische Kostenzuordnung. Die Stärken der typischen IFCN-Betriebe liegen aufgrund der Erfassungstiefe in der Ermittlung realitätsnaher betrieblicher Anpassungsreaktionen. Es sind jedoch keine Rückschlüsse auf Regions- bzw. Mitgliedsländerebene ableitbar, da typische Betriebe aufgrund ihrer geringen Anzahl nicht repräsentativ sind.

Daher ist das Ziel dieser Arbeit, ein methodisches Konzept zur Verknüpfung der einzelbetrieblichen Datensätze des FADN und IFCN für eine verbesserte Politikfolgenabschätzung zu entwickeln. Durch Verknüpfung sollen die komparativen Vorzüge beider Datenquellen genutzt werden. Dazu wurden zwei Verknüpfungsansätze entwickelt:

a) die Nutzung von FADN zur Einordnung und Auswahl der IFCN-Betriebe und

b) die Nutzung von IFCN-Daten zur Aufbereitung von FADN-Daten, um somit die FADN-Betriebe für Kalkulationen mit dem Simulationsmodell TIPI-CAL verwenden zu können.

Weil der Milchmarkt eine hohe agrarpolitische und wirtschaftliche Bedeutung in der EU aufweist und für diesen Bereich eine ausreichende einzelbetriebliche Datenbasis zur Verfügung steht, erfolgt die empirische Analyse dieser Arbeit am Beispiel der EUMilchmarktpolitik.

Als Grundlage für die durchzuführende Analyse wird zunächst ein kurzer Überblick über die Bedeutung und Struktur der europäischen Milchwirtschaft, die für die Politikfolgenabschätzung verwendeten einzelbetrieblichen Modelle und Datenbasis gegeben. Die Analyse der vorhandenen Datengrundlagen hat ergeben, dass FADN und IFCN besonders geeignet sind, um im empirischen Teil dieser Arbeit kombiniert zu werden. Daher wird im Folgenden aufgezeigt, wie FADN und IFCN für Analysen zur Liberalisierung des EUMilchmarktes bisher eingesetzt worden sind und dargelegt, wo Potenziale und mögliche Fehler in den Ergebnissen bzw. Begrenzungen der Aussagekraft liegen. Dies erfolgt, um anschließend zu prüfen, ob die kombinierte Anwendung zu besseren Ergebnissen führt. 
Bezüglich des ersten Verknüpfungsansatzes wird zunächst gezeigt, wie IFCN-Betriebe in die Grundgesamtheit einzuordnen sind. Es wird ermittelt, welchen Anteil der Betriebe und der Milchmenge die bisher im IFCN als typisch ausgewählten Betriebe repräsentieren. Dazu werden die IFCN- und FADN-Daten anhand struktureller Kennzahlen verglichen und darauf aufbauend die FADN-Betriebe ausgewählt, die den IFCN-Betrieben strukturell am ähnlichsten sind. Die Ähnlichkeit zwischen den Betrieben wird anhand der euklidischen Distanz ermittelt. Mit Hilfe der Berechnung unterschiedlicher Stichprobenzusammensetzungen wird versucht, eine dem typischen Betrieb möglichst ähnliche Gruppe von FADNBetrieben zu ermitteln. Um eine möglichst hohe Ähnlichkeit der Betriebe zu erzielen, wird die Anzahl FADN-Vergleichsbetriebe zunächst auf 25 begrenzt. In die Untersuchung werden Betriebe aus Regionen in Deutschland, Schweden und Spanien einbezogen, in denen die Milcherzeugung eine hohe Bedeutung für die regionale landwirtschaftliche Produktion hat und die ein relativ breites Betriebsgrößenspektrum aufweisen.

Die Berechnungen zeigen, dass der prozentuale Anteil der Milchproduktion pro Land, der von den IFCN-Betrieben repräsentiert wird, zwischen 1,2 und 8,8 \% variiert. Der prozentuale Anteil der abgebildeten Betriebe in den untersuchten Regionen variiert in Deutschland zwischen 2,6 (35-Kuhbetrieb in Bayern) und 3,8 \% (68-Kuhbetrieb in Niedersachsen).

Da die maximale Anzahl von 25 FADN-Vergleichsbetrieben gewählt worden ist, wurde im zweiten Schritt geprüft, in wie weit sich die Repräsentativität der Betriebe erhöht, wenn eine größere Anzahl von Vergleichsbetrieben einbezogen wird. Die Repräsentativität des typischen niedersächsischen Betriebs bezogen auf die Grundgesamtheit erhöht sich beispielsweise auf $8 \%$, wenn anstelle von maximal 25 Betrieben 60 Betriebe mit der höchsten Ähnlichkeit zum typischen Betrieb zugeordnet werden. Wenn anstelle von 25 Betrieben 150 FADN-Betriebe mit der höchsten Ähnlichkeit einbezogen werden, erhöht sich die Repräsentativität des typischen 68-Kuhbetriebes in der Region auf 20 \%. Das euklidische Distanzmaß erhöht sich dabei und die Standardabweichung steigt bei zunehmender Repräsentativität erwartungsgemäß an. Bei einer ungünstigeren Ähnlichkeitszahl wird die Gruppe inhomogener.

In einem weiteren Schritt wird geprüft, ob die IFCN-Betriebe nicht nur hinsichtlich ihrer strukturellen Eigenschaften, sondern auch im Hinblick auf ihre wirtschaftliche Leistungsfähigkeit typisch für ihre Größenklasse in der Region und im Land sind. Es wird analysiert, für wie viele Betriebe aus der Grundgesamtheit Rückschlüsse aus den IFCNAnalysen gezogen werden können. Zur Einordnung der IFCN-Betriebe werden als Indikatoren die Vollkosten und die Kosten aus der Gewinn- und Verlust-Rechnung herangezogen, die einen Rückschluss auf kurz-/mittel- und langfristige Einkommenseffekte gewährleisten. Für jeden FADN-Betrieb werden diese Kosten ermittelt, auf einer kumulierten 
Verteilungskurve aufgezeigt und die typischen IFCN-Betriebe eingeordnet. Dieser Schritt erfolgt anhand von zwei Gruppen von FADN-Vergleichsbetrieben.

Die statistische Einordnung der IFCN-Betriebe anhand ihrer Kosten verdeutlicht, dass der norddeutsche 68-Kuhbetrieb gut vergleichbar ist mit der entsprechenden FADNBetriebsgruppe und einen durchschnittlich leistungsstarken 60- bis 70-Kuhbetrieb in Norddeutschland (Niedersachsen/Schleswig-Holstein) darstellt. Der typische bayerische 35-Kuhbetrieb weist bedeutend höhere Kosten auf als die zu vergleichende FADNBetriebsgruppe und stellt das obere Viertel auf der Verteilungskurve dar. Dies trifft für beide Kostenindikatoren zu, wobei der Vollkostenindikator an der obersten Grenze liegt und nur ca. $10 \%$ der Betriebe mit den höchsten Produktionskosten widerspiegelt. Die Spannbreite der Produktionskosten ist bei den FADN-Vergleichsbetrieben zum 35Kuhbetrieb größer als bei den Vergleichsbetrieben zum 68-Kuhbetrieb, bedingt durch andere Betriebszweige und unterschiedlich hohe Nebenerlöse. Dieser Vergleich der Daten typischer Betriebe mit den Struktur- und Leistungsdaten aus dem FADN liefert somit ebenfalls wertvolle Informationen zu der Frage, wie gut der IFCN-Betrieb die regionale Grundgesamtheit abbilden kann. Diese Einschätzungen sind für die Korrektur der Inputdaten typischer Betriebe und für die Neudefinition typischer Betriebe nützlich.

Wie die zuvor erläuterten Analysen zeigen, lässt sich mit Hilfe des euklidischen Distanzmaßes feststellen, ob zu den typischen IFCN-Betrieben ähnliche Betriebe in der FADNStichprobe vorliegen und wie viele Betriebe aus der Grundgesamtheit von diesen repräsentiert werden. Hierbei ist jedoch zu bedenken, dass die mit Hilfe der Einordnung ermittelte Repräsentanz der IFCN-Betriebe je nach gewählter Ähnlichkeitszahl stark variieren kann und es keine objektive Regel gibt, welches Distanzmaß gerade noch akzeptabel ist. Von daher stellt sich die Frage, ob der FADN-Datensatz nicht schon besser bei der Auswahl der typischen Betriebe genutzt werden sollte, als nachher festzustellen, wie repräsentativ die mit Hilfe von Beratern festgelegten typischen Betriebe tatsächlich sind. Deshalb wurde ergänzend ein Verfahren entwickelt, wie die Auswahl typischer IFCN-Betriebe auf Basis statistischer Daten (FADN-Daten) erfolgen kann, um eine bessere Repräsentativität zu erhalten. Als Methode zur Auswahl typischer IFCN-Betriebe wurde die Clusteranalyse gewählt mit dem Ziel herauszufinden, welche Anzahl IFCN-Betriebe pro Land nötig ist, um in der Vielfalt der Strukturen und Betriebskonstellationen die bedeutendsten Betriebstypen im Hinblick auf die Produktionsmenge abzudecken. Aus den gebildeten Clustern lässt sich dann ableiten, welche Strukturvorgaben für die typischen IFCN-Betriebe zu treffen sind.

Die Methodik der Clusteranalyse wird am Beispiel deutscher (spezialisierter) Milchviehbetriebe aus dem FADN-Datensatz aufgezeigt. Es wird das Ward-Verfahren angewendet, welches Cluster mit annähernd gleichen Besetzungszahlen abbildet. Zur regionalen Abgrenzung wird Deutschland in drei Regionen untergliedert (Region Nord-West, Mitte-Süd, 
Ost). Es werden fünf Klassifizierungsmerkmale berücksichtigt, die die Betriebsstruktur, die Intensität der Produktion und die Produktionsleistung berücksichtigen. Das beschriebene Clusterverfahren wird auf die ausgewählten 1.730 Milchviehbetriebe aus dem deutschen FADN angewendet. Eine maximale Begrenzung auf 15 Cluster ergab eine Gruppierung von 12 Clustern je Region, die relativ detailgenaue Werte lieferten. Sortiert man die 12 Cluster je Region nach der Herdengröße, so ergeben sich in den Regionen Nord-West, Mitte-Süd und Ost jeweils vier bis sechs Gruppen mit eins bis fünf Clustern. Die Cluster mit ähnlicher Herdengröße zeigen deutliche Unterschiede hinsichtlich der Naturalleistung (Milchleistung), Intensität der Produktion (Viehbesatzdichte) und Ausstattung mit Fläche. Die Bedeutung der einzelnen Cluster im Hinblick auf die nationale Milchproduktion variiert z.B. in Nord-West-Deutschland zwischen 0,2 und 9 und die Anzahl repräsentierter Milchviehbetriebe variiert zwischen 350 und 2900 Betrieben aus der Grundgesamtheit. Die Mittelwerte der Cluster dienen als Charakteristika zur Klassifizierung typischer IFCN-Betriebe. Die Streubreite in den Regionen soll Rückschlüsse über die Homogenität der Cluster ermöglichen. Es wurden mittels Clusteranalyse 36 Cluster von typischen Betrieben gebildet. Im Rahmen des IFCN/agri benchmark lässt sich diese Zahl nicht umsetzen, weil sie zu groß ist. Daher wird vorgeschlagen, anhand der Cluster mit den höchsten regionalen Produktionsanteilen mit Hilfe von Beratern zu einer Auswahl von Betrieben zu kommen, die die Regionen am besten charakterisieren.

Beim zweiten Verknüpfungsansatz ist es das Ziel, eine größere Anzahl von FADNBetrieben nutzbar zu machen für differenzierte Simulationsrechnungen im Modell TIPICAL unter Berücksichtigung betrieblicher Anpassungsreaktionen.

Da die FADN-Daten primär zur Einkommensanalyse erhoben werden, benötigt man weitere Informationen, um mit FADN-Daten im TIPI-CAL Modell Berechnungen durchführen zu können. Für Analysen im TIPI-CAL Modell ist es notwendig, dass diejenigen Variablen, die im FADN nicht vorhanden sind, ergänzt werden. Daher wird zwischen Variablen unterschieden, die a) im FADN vorhanden sind, b) nicht im FADN vorhanden sind und z.B. durch die IFCN-Variable ersetzt werden und c) die sowohl aus dem IFCNDatensatz als auch aus dem FADN-Datensatz generiert werden. Die detaillierte Anreicherung und Korrektur der FADN-Variablen erfolgt aus Konsistenzgründen und im Hinblick auf das Ziel dieser Arbeit vornehmlich mit Daten aus dem IFCN. Um die Fehlerrisiken bei der Ergänzung des FADN-Datensatzes zu reduzieren, kommmt u. a. die Koeffizientenschätzmethode (HINRICHS und HAIMBÖCK, 1980) zur Anwendung.

Die Verknüpfung wird anhand eines Beispielbetriebs aufgezeigt. Es werden exemplarisch die Auswirkungen des MTR-Beschlusses und eines angenommenen Ausstiegs aus der Milchquotenregelung für den Zeitraum 1999 bis 2008 berechnet. Das Referenzszenario, das zum Vergleich der beiden Politiken genutzt wird, beinhaltet die Beibehaltung der „Agenda 2000“-Politik. 
Um die Leistungsfähigkeit des Verknüpfungsansatzes beurteilen zu können, werden die Simulationsergebnisse des modifizierten FADN-Betriebes mit denen des entsprechenden IFCN-Betriebes verglichen. Erwartungsgemäß zeigen die FADN- und IFCN-Betriebe ähnliche Ergebnisse. Für die Entwicklung des betrieblichen Gewinns (1999 bis 2008) unter Agenda-Bedingungen als auch im Hinblick auf den MTR-Beschluss sowie einen Milchquotenausstieg werden plausible Ergebnisse erzielt. Die errechneten Anpassungspotenziale liegen in beiden Betrieben auf ähnlichem Niveau. Die FADN-Betriebsgruppe verzeichnet niedrigere Kosten als der IFCN-Betrieb aufgrund der unterschiedlichen Bewertung der Nebenerlöse und der kalkulatorischen Faktorkosten.

Es bietet sich an, die entwickelte Verknüpfung in ein Computerprogramm zu überführen, um ohne viel Aufwand eine größere Zahl von FADN-Betrieben mit Hilfe differenzierter Simulationsmodelle analysieren zu können. Damit wären auch Aussagen über die Varianz der Einkommenseffekte und die individuelle Betroffenheit der Betriebe möglich. Durch die Verknüpfung der Daten und Informationen aus den beiden Konzepten ist es darüber hinaus möglich, bei der Modellierung realitätsnahe Anpassungsreaktionen in einer größeren Zahl von Betrieben zu berücksichtigen. Das ist vor allem deshalb wichtig, weil Politikfolgenabschätzungen ohne Berücksichtigung realitätsnaher, betrieblicher Anpassungsreaktionen zu Fehleinschätzungen führen können. Das gilt insbesondere für komplexe Fragestellungen, bei denen betriebliche Anpassungen in größerem Ausmaß zu analysieren sind; für weniger komplexe Fragestellungen kann demgegenüber eine einfache komparativ-statische Analyse ausreichend sein. In Bezug auf die Erfassung der Nebenerlöse und des Arbeitsinputs als auch zur Übertragung betrieblicher Anpassungsreaktionen auf die FADN-Betriebsgruppe wird empfohlen, die Informationsbasis des FADN mit ExpertenKnow-how aus der Region ,abzurunden“ und die resultierenden Simulationsergebnisse im iterativen Prozess mit den zuständigen Beratern aus dem IFCN zu diskutieren und zu bewerten. 


\section{Literaturverzeichnis}

American Agricultural ECONOMic Assoziation (2000) Commodity Costs and Returns Estimation Handbook - A report of the AAEA Task force on Commodity Costs and Returns, Ames, Iowa, 1

ADR Arbeitsgemeinschaft Deutscher Rinderzüchter e. V. (2001) Rinderproduktion in der Bundesrepublik Deutschland 2000, Ausgabe 2001

Amelung C (1999) Vergleichende Analyse der Produktionskosten ausgewählter Marktfruchtbetriebe in Sachsen-Anhalt und Ungarn. Diplomarbeit, Göttingen, S. 87-93

ARFINI F (2001) Mathematical programming models employed in the analysis of the common agricultural policy, working paper n. 9, Department of Economics, University of Parma. Italy

ARfini F (2002) Mathematical Programming models employed in the analysis of the Common Agricultural Policy, working paper n. 9, Instituto Nationale di Economia Agraria (INEA)

Arfini F, Conforti P, Donati M, Schokai P, Scopolla M, Sorrentino A (2002) Reforming the EU milk quota regime: The effect on Italian dairy farming. National Institute of Agricultural Economics (INEA), Italy

BacKHAus K et al. (1996) Multivariate Analysemethoden. 8. Auflage, Springer-Verlag, Berlin

Benjamin C, Gohin A, Guyomard H (1999) The future of European Union dairy policy. Canadian Journal of Agricultural Economics (CAN). Vol. 47, No. 5, pp. 91-101

Bertelsmeier, M. (2005) Analyse der Wirkungen unterschiedlicher Systeme von direkten Transferzahlungen unter besonderer Berücksichtigung von Bodenpacht- und Quotenmärkten. Schriftenreihe des Bundesministeriums für Ernährung, Landwirtschaft und Forsten. Angewandte Wissenschaft, Heft 510. Münster: Landwirtschaftsverlag.

Bertelsmeier M, Kleinhanss W, Offermann F (2003) Aufbau und Anwendung des FAL-Modellverbunds für die Politikberatung. Agrarwirtschaft 52, Heft 4

BORTz J (1989) Statistik für Sozialwissenschaftler. 3. Auflage, Berlin

BORTZ J (1993) Clusteranalyse, Statistik für Sozialwissenschaftler, Springer-Verlag, Berlin/Heidelberg/New York

BORTZ J (2005) Statistik für Human- und Sozialwissenschaftler, 6. Auflage, 2005

Bouamra-Mechemache Z, DartiguesS K, Requillar V (2001) Réforme de la politique de l’Union Européenne : analyse de scénarios. INRA, Unité d’Économie et Sociologie Rurales, Institut d'Economie Industrielle, Toulouse

Brümmer B, von Cramon-Taubadel S, Kösters U, Meyer J (2002) Evaluation of the Work on Agricultural Sector Modelling in EUROSTAT, Tender Document No. 1999/S 228-158180/EN, Final Report, July 2002 
BundESGESETZBLATT vom 05.09.1955, Teil 1, S. 565

BunDESGESETZBLATT vom 25.03.1957, Teil 2, S. 766

BUNDESMINISTERIUM FÜR ERNÄHRUNG, LANDWIRTSCHAFT UND FORSTEN (1992) Agrarbericht 1992. Agrar- und ernährungspolitischer Bericht der Bundesregierung. Bonn

BUNDESMINISTERIUM FÜR ERNÄHRUNG, LANDWIRTSCHAFT UND FORSTEN (1993) Agrarbericht 1993. Agrar- und ernährungspolitischer Bericht der Bundesregierung. Bonn

BUNDESMINISTERIUM FÜR ERNÄHRUNG, LANDWIRTSCHAFT UND FORSTEN (1993) Buchführung der Testbetriebe - Ausführungsanweisungen zum BMVEL-Jahresabschluss, Stand: 01.06.1993

BUNDESMINISTERIUM FÜR ERNÄHRUNG, LANDWIRTSCHAFT UND FORSTEN (1993) Statistisches Jahrbuch über Ernährung, Landwirtschaft und Forsten. Landwirtschaftsverlag, Münster-Hiltrup, S. 147

BUNDESMINISTERIUM FÜR ERNÄHRUNG, LANDWIRTSCHAFT UND FORSTEN (2000) Zum Agrarbericht 2000: Buchführungsergebnisse der Testbetriebe, Reihe: Daten-Analysen. Bonn, S. $110 \mathrm{ff}$.

BUNDESMINISTERIUM FÜR VERBRAUCHERSCHUTZ, ERNÄHRUNG UND LANDWIRTSCHAFT (2003) Ernährungs- und agrarpolitischer Bericht der Bundesregierung. Berlin, S. 26 und 154

BUNDESMINISTERIUM FÜR VERBRAUCHERSCHUTZ, ERNÄHRUNG UND LANDWIRTSCHAFT (2004) Zum Ernährungs- und agrarpolitischen Bericht 2004: Buchführungsergebnisse der Testbetriebe; Reihe: Daten-Analysen, WJ 2002/03 bzw. KJ 2002. Bonn

Burrell A (1995) Konzeption und Leistung von Agrarsektormodellen. Teil 1, S. 12, Fachbereich Agrarökonomie der Landwirtschaftlichen Universität Wageningen, Niederlande, EUROSTAT

Chatellier V, Colson F, Arnaud F, Guedson JC, Kumpf M, Legendre J, Perrot C (1997) Typologie: Exemples des exploitations laitières, INRA Prod. Anim., 1997, 10 (3), 227-240 Agreste, 1997, Les Cahiers No. 22-23, 11/97, 182 p.

Chatellier V, Colson F, Fuentes MA, VArd T (2000) Les exploitations d' élevage herbivore dans l'Union européenne. INRA Prod. Anim., 13, 201-213. INRA Productions Animales, Juli 2000

ChATELlier V, JACQUeRIE V (2003): Les exploitations laitières dans l'union européenne, Situation actuelle et perspectives face aux réformes de la PAC, Rapport final, INRA-ESR Nantes, Frankreich

CHRISTOFFers K (2001) Analyse der Produktionskosten ausgewählter Milchviehbetriebe in Deutschland und den USA. Diplomarbeit, Göttingen 
Colman D (2002) Phasing out of milk quotas in the EU. University of Manchester, Centre for Agriculture, Food and Resource Economics. Report submitted to Department for Environment, Food and Rural Affairs (DEFRA/UK). (Download: http://www.Defra.gov.uk/esg/economics/Milkquota/index.htm)

Colman D, Burton MP, RigBy DS, Franks JR (1998) An economic evaluation of the UK milk quota system. Report commissioned by MAFF, the Welsh Office Agricultural Department, Department of Agriculture for Northern Ireland and Scottish Office of Agriculture, Environment and Fisheries, CAFRE, School of Economic Studies, University of Manchester

Colman D, Burton MP, RigBy DS, Franks JR (2001) Projections of Structural Change in UK Dairy Production. Report to the Department for Environment, Food and Rural Affairs, CAFRE School Economic Studies, University of Manchester

Colson F, Chatellier RV, Fuentes MA (1999) Typologie des systèmes d'élevage herbivoredans l'Union Européenne, INRA Nantes, Commission Européenne, DG VI Agriculture

Commission of the European Communities (2002) Report on milk quotas. Commission working document Brussels/Belgium

(Download: http://europa.eu.int/comm/agriculture/mtr/milkrep/text_en.pdf)

DeBLitz C (1993) Internationaler Vergleich von Systemen extensiver tiergebundener Grünlandnutzung - produktionstechnische und ökonomische Analyse, Wettbewerbsfähigkeit, internationale Übertragbarkeit. Dissertation, Göttingen, 1993

DeBlitz C et al. (1998) International Farm Comparison Network (IFCN) - Ziele, Organisation, erste Ergebnisse für die Milchproduktion. Gekürzte Fassung des IFCNBerichts 1/98, Institut für Betriebswirtschaft, Agrarstruktur und ländliche Räume der Bundesforschungsanstalt für Landwirtschaft Braunschweig

DeBlitz C et al. (1998) The International Farm Comparison Network (IFCN). Objectives, Organisation, First Results on Dairy Production [online]. Bundesforschungsanstalt für Landwirtschaft, Braunschweig http://www.bal.fal.de/download/r1e-41.pdf

Deeken E, Hemme T (2002) Milchmarktpolitik 2008 - Auswirkung eines Ausstiegs aus der Milchquotenregelung auf typische Milchviehbetriebe in Deutschland, Arbeitsbericht 5/02, Institut für Betriebswirtschaft, Agrarstruktur und ländliche Räume der Bundesforschungsanstalt für Landwirtschaft, Braunschweig

EARl O HeAdy O (1950/60) His impact on Agricultural Economics. In: LANGLey J, Vocke G, Whiting L (1994): Iowa State Press

EUROPÄISCHE KOMMISSION, GENERALDIREKTION LANDWIRTSCHAFT (2000) Gemeinschaftsausschuss des Informationsnetzes landwirtschaftlicher Buchführungen (INLB): Definition der Variablen in den INLB-Standardergebnissen; Brüssel 
EUROPÄISCHE KOMMISSION, GENERALDIREKTION LANDWIRTSCHAFT (2006) Gemeinschaftsausschuss des Informationsnetzes landwirtschaftlicher Buchführungen (INLB): Costs of production for milk in the European Union, Period 19972003; Brüssel

EUROPÄISCHE KOMMISSION, GENERALDIREKTION LANDWIRTSCHAFT UND LÄNDLICHE ENTWICKLUNG (2006) Informationen zur Agrarreform, http://europa.eu.int/agriculture/index_en.htm

EUROPÄISCHE KOMMISSION (2008) Commission staff working document, Impact Assessment, Brüssel

EUROSTAT (1989) Handbuch zur Landwirtschaftlichen und Forstwirtschaftlichen Gesamtrechnung, Luxemburg, S. 7

EUROSTAT (1995) Methodological documentation (Rev. 1), Vol. 1: Basics, EUROSTAT, Luxemburg

EUROSTAT (1997) Farm Structure Survey

EUROSTAT (2006) Schätzungen GD Landwirtschaft, Warenbestände EAGFL

FENNECKER A (2002) Tiergerechte Schweinehaltung unter ethischen, rechtlichen und ökonomischen Aspekten. Dissertation, Göttingen

Food And Agriculture Organization of the United Nations (2006) FAOSTAT, Production and Trade, http://faostat.fao.org

Gabler WirtschaftSleXiKon (2000) 15. Auflage, September 2000.

Gesetz über Agrarstatistiken (Agrarstatistikgesetz-AgrStatG) in der Fassung der Bekanntmachung vom 25. Juni 1998, BGBI. I, S. 1635

GESELLSCHAFT FÜR WIRTSCHAFTS- UND SOZIALWISSENSCHAFTEN DES LANDBAUES (1984) Einkommen in der Landwirtschaft: Entstehung, Verteilung, Verwendung und Beeinflussung, anlässlich der Jahrestagung der Gesellschaft für Wirtschafts- und Sozialwissenschaften des Landbaues in Hannover, 1983. (Hrsg.) GrossKOPF W Mitarbeiter: ALBERS W. Landwirtschaftsverlag Münster-Hiltrup

GoERTZ D (1998) Einkommenswirkungen der Agenda 2000 auf typische milchviehhaltende Betriebe in Europa. Diplomarbeit, Göttingen

Goertz D (1999) Produktionskosten der Milcherzeugung in Deutschland. Arbeitsbericht 3/99, Institut für Betriebswirtschaft, Agrarstruktur und ländliche Räume der Bundesforschungsanstalt für Landwirtschaft

GRIEPENKERL W (1998) Agrarstatistik auf neuen Wegen, S. 661-670

HASSKAMP H (1976) Programm und Organisation der Agrarberichterstattung. In: STATISTISCHES BunDESAMT (Hrsg.): Wirtschaft und Statistik, Heft 8, S. 469-475

HASSKAMP, H. (1979) Konzept der Landwirtschaftszählung 1979. In: STATISTISCHES BuNDESAMT (Hrsg.): Wirtschaft und Statistik, Heft 4, S. 245-253 
HAUPTVERBAND DER LANDWIRTSCHAFTLICHEN BUCHSTELLEN UND SACHVERSTÄNDIGEN E. V. (HLBS) (1995) Bewertung in der landwirtschaftlichen Buchführung. 4. Auflage. Schriftenreihe, Heft 88

Helming J, Peerlings J (2002) The impact of milk quota abolishment on Dutch agriculture and economy - Applying an agricultural sector model integrated into a mixed input-output model. Paper presented on the 10. EAAE-Conference, August 28-31 Zaragoza, Spain

Hemme T (2000) Ein Konzept zur international vergleichenden Analyse von Politik- und Technikfolgen in der Landwirtschaft. Landbauforschung Völkenrode, Sonderheft 215, Braunschweig

Hemme T (2000) IFCN Dairy Report 2000. IFCN/Global Farm, Braunschweig

HEMmE T (2001) IFCN Dairy Report 2001. IFCN/Global Farm, Braunschweig

Hemme T (2002) IFCN Dairy Report 2002, IFCN/Global Farm, Braunschweig

HEMme T (2003) IFCN Dairy Report 2002, IFCN/Global Farm, Braunschweig

Hemme T et al. (1997) Ein Modell zur Politik- und Technikfolgenabschätzung für typische Betriebe im internationalen Vergleich. Arbeitsbericht 2/97, Institut für Betriebswirtschaft, Agrarstruktur und ländliche Räume der Bundesforschungsanstalt für Landwirtschaft, Braunschweig

HEMme T et al. (2002) IFCN Dairy Report 2002. IFCN/Global Farm, Braunschweig, S. 108-109, JÄGersberg P: Cost of Production Analysis cariied out with FADN and IFCN

Hemme T et al. (2002, 2003) IFCN Dairy Report. IFCN/Global Farm, Braunschweig

HeMme T, Christoffers K, DEEKEN E (eds) (2004) IFCN dairy report 2004: For a: better understanding of milk production world-wide. Braunschweig: Global Farm GbR, 152 p

Hemme T, HeinRich I, IsERmeyer F (1997) Die Wettbewerbsfähigkeit von Milchkuhbetrieben in Ost- und Westdeutschland im internationalen Vergleich. Arbeitsbericht 3/97, Institut für Betriebswirtschaft der Bundesforschungsanstalt für Landwirtschaft Braunschweig

HENNESSY T (2000) Modelling farmer response to policy reform: An irish example. Paper presented at the $65^{\text {th }}$ EAAE Seminar. Bonn

HENNESSy T (2000) An analysis of farm margins and structures to 2007. AESI and ASA joint meeting $6^{\text {th }}$ April 2000. Rochstown Park Hotel. Cork

Hennessy T (2001) Paper presented at the Irish Grasslands Association Annual Conference. Silversprings Hotel. Cork/Ireland.

HENNESsy T et al. (2000) The changing structure and production potential of Irish dairy farming in the context of quota abolition. Paper presented at the Agri-Food Economics Conference 2000. Green Isle Hotel. Dublin 
HenRICHSMEYER W, DE HAEN H (1972) Zur Konzeption des Schwerpunktprogrammes der Deutschen Forschungsgemeinschaft „Konkurrenzvergleich landwirtschaftlicher Standorte“. Agrarwirtschaft 21, S. 141-152

HENRICHSMEYER W et al. (1992) Endbericht (Modellbeschreibung) zum Forschungsvorhaben „Aufbau eines computergestützten, regionalisierten Agrar- und Umweltinformationssystems für die Bundesrepublik Deutschland“. Bonn

HENRICHSMEYER W et al. (1996) Endbericht zum Kooperationsprojekt Entwicklung des gesamtdeutschen Agrarsektormodells RAUMIS96, Forschungsvorhaben - 94 HS 021 - des BMELF. Bonn/Braunschweig

HENRICHSMEYER W, KREINS P (1992) Endbericht (EDV-Beschreibung) zum Forschungsvorhaben „Aufbau eines computergestützten regionalisierten Agrar- und Umweltinformationssystems für die Bundesrepublik Deutschland“, Bonn

HenRichsmeyer W, Witzke HP (1991) Agrarökonomische Grundlagen. Agrarpolitik Band 1, Bonn

Hertel TW (1997) Global Trade Analysis: Modelling and Applications, Cambridge University Press

HiNRICHS P, HAIMBÖCK H (1980) Erfahrungen aus der Anwendung eines mikroökonomisch orientierten Modells zur sektoralen und betriebsgruppenspezifischen Einkommensprojektion., Schriften der Gesellschaft für Wirtschafts- und Sozialwissenschaften des Landbaus e. V., Band 17, Prognose und Prognosekontrolle

HINRICHS P, JÄGERSBERG P (2001) ELPEN Zwischenbericht („progress report“) für die EU-Kommission. Unveröffentlichtes Dokument

HINRICHS P, JÄGERSBERG P (2002) ELPEN Zwischenbericht („progress report“) für die EU-Kommission. Unveröffentlichtes Dokument

HINRICHS P, JÄGERSBERG P (2003) ELPEN Zwischenbericht („progress report“) für die EU-Kommission. Unveröffentlichtes Dokument

HolzNeR J (2003) Eine Analyse der internationalen Wettbewerbsfähigkeit der Milcherzeugung an ausgewählten Standorten in Ostdeutschland, der Tschechischen Republik und Estland. Dissertation

HowitT RE (1995) Positive Mathematical Programming. American Journal of Agricultural Economy, Vol. 77, S. 329-342

http://europa.eu.int/comm/agriculture/rica/methodology2_de.cfm http://europa.int/comm/agriculture/rica/methodology2_de.cfm

HÜtTel S, Kleinhanß W, OfFermann F (2005) Impacts of decoupling and milk quota trade on the French and German dairy sectors. In: 11. Congress European Association of Agricultural Economics "The future of rural Europe in the global agri-food system": Copenhagen, Denmark, 24-27 August 2005

IserMeYer F (1988) Produktionsstrukturen, Produktionskosten und Wettbewerbsstellung der Milcherzeugung in Nordamerika, Neuseeland und der EG. Dissertation 
Isermeyer F, Brockmeier M, Gömann H, Hargens R, Klepper R, Kreins P, Offermann F, Osterburg B, Pelikan J, Salamon P, Thiele H (2006) Analyse politischer Handlungsoptionen für den Milchmarkt. Landbauforschung Völkenrode Sonderheft Nr. 300, Braunschweig: FAL

Isermeyer F, Deblitz C, Hemme T, Plessmann F (2003) 20 Fragen und Antworten zum IFCN. Institut für Betriebswirtschaft, Agrarstruktur und ländliche Räume der Bundesforschungsanstalt für Landwirtschaft und Global Farm GbR, Braunschweig (aktualisierte Fassung)

JACOBS A (1998) Paralleler Einsatz von Regionen- und Betriebsgruppenmodellen in der Agrarsektoranalyse. Institut für Betriebswirtschaft, Agrarstruktur und ländliche Räume der Bundesforschungsanstalt für Landwirtschaft. Dissertation

JÄGERSBERG P (2002) Costs of Production for Milk in the European Union based on FADN data, PACIOLI 10: European Farmers and the growing of data, S. 176. Verfasser des Bandes zur 10. PACIOLI Jahrestagung: Krijn J. Poppe (LEI), Andrea Povellato (INEA, Karlijn Krijgsman (LEI)

JÄGERSBERG P (2002) Cost of production analysis carried out with FADN and IFCN, erschienen im IFCN Dairy Report 2002

JÄGERSBERg P, PERRoT C (2001) TRANSFORM - Excel-Tool zur Übertragung von französischen „Cas types“ in das Netzwerk typischer IFCN-Betriebe

JANSSONO T (2002) Consequences for agriculture, consumers and taxpayers of abolishing milk quotas in the EU. Paper presented on the 10. EAAE-Conference, August 28-31, Zaragoza, Spain

JAYET PA (2002) Retrait de terres et subsidiarité dans le cadre de la Politique Agricole Commune. Recherches Economiques de Louvain. Vol 58 (4), pp. 407-428

Jogeneel R (2005) Report on modeling milk and dairy industry in the new Member States, INRA Toulouse, Frankreich, 31.12.2005

KLEINHANSS W (2000) Betriebsgestützte Sektormodellierung oder sektorkonsistente Betriebsmodelle - Wohin steuert die Betriebsmodellierung? Überarbeitete Fassung des im agrarökonomischen Kolloquium der MLU und des IAMO Halle am 5. Juli 2000 gehaltenen Vortrages.

(Download: http:// www.bal.fal.de/download/betriebsmodellierung.pdf)

KLEINHAnss W et al. (1998) Auswirkungen der Agenda 2000 auf die deutsche Landwirtschaft - Eine modellgestützte Folgenabschätzung auf Markt-, Sektor-, Regionsund Betriebsebene. Braunschweig. Arbeitsbericht 02/98. Institut für Betriebswirtschaft der Bundesforschungsanstalt für Landwirtschaft, Braunschweig

Kleinhanss W et al. (2001) Mögliche Auswirkungen eines Ausstiegs aus der Milchquotenregelung für die deutsche Landwirtschaft. Institut für Betriebswirtschaft, Agrarstruktur und ländliche Räume der Bundesforschungsanstalt für Landwirtschaft, Braunschweig 
KLeinhanss W et al. (2002) Phasing out of milk quotas - Possible impacts on German agriculture. Arbeitsbericht 01/02. Institut für Betriebswirtschaft, Agrarstruktur und ländliche Räume der Bundesforschungsanstalt für Landwirtschaft (Download: http://www. bal.fal.de/en/publications.htm)

KLEINHANSS W et al. (2002) Szenarien zur Entkopplung produktionsgebundener Prämien. Partielle Umwidmung von Rinder- und Milchprämien in Grünlandprämien. Arbeitsbericht 2/02, Institut für Betriebswirtschaft, Agrarstruktur und ländliche Räume der Bundesforschungsanstalt für Landwirtschaft, Braunschweig (Download: http://www.bal.fal.de)

KLEINHANSS W et al. (2003) „LIST OF SHEET TABLES“. FADN-Variablenliste. Unveröffentlichtes Dokument

KOMMISSION DER EUROPÄISCHEN GEMEINSCHAFTEN (1982) Das INLB, Resultate auf Mikroplanfilm. Brüssel

KÜPKer B, HÜtTel S, Kleinhanß W, OfFermann F (2006) Assessing impacts of CAP reform in France and Germany. Agrarwirtschaft, Vol. 55, No. 5/6, S. 227-237

KURATORIUM FÜR TECHNIK UND BAUWESEN IN DER LANDWIRTSCHAFT: Verschiedene Jahrgänge

LANDWIRTSCHAFTSKAMMER HANNOVER UND WESER-EMS (2004) RichtwertDeckungsbeiträge 2004. Sonderheft B/2004

LANDWIRTSCHAFTSKAMmER SchleswiG-Holstein (2001) Rinder-Report 2001. Ergebnisse der Rinderspezialberatung in Schleswig-Holstein, Betriebswirtschaftliche Mitteilungen der Landwirtschaftskammer Schleswig-Holstein, Abteilung Betriebsführung und Beratung. Kiel

Lehmann B, Eggenschwiler C, Weber M, Wolf HP, Dumondel M, Gerwig C, AlBISSER G, HEss R (2000) Evaluation der Milchkontingentierung. Vorstudie mit Schwerpunkt Wirkungsanalyse, Schlussbericht zu Händen der Hauptabteilung Produktion und Internationales, Bundesamt für Landwirtschaft, Bern; Eidgenössische Technische Hochschule Zürich (ETH), Institut für Agrarwirtschaft (IAW), Gruppe Betriebswirtschaft und Ökonomie des ländlichen Raumes. Zürich

LiLLARD P (1995) A Documentation of the Revised European Simulation (ESIM) Modelling Framework. United States Department of Agriculture Economic Research Service (USDA/ERS), Washington

LIPS M, RIEDER P (2002) Endogenous adjusted production quotas - The abolishment of the raw milk quota in the European Union. Paper presented on the 10. EAAEConference, August 28-31, Zaragoza, Spain

Milk Development Council (2006) EU Milk Production, http://www.mdcdatum.org.uk

Milligan G-W (1981) A review of Monte Carlo tests of cluster analysis. Mult. Beh. Res., 24 (1981) 379-407 
NieBerg H (1997) Produktionstechnische und wirtschaftliche Folgen der Umstellung auf ökologischen Landbau - empirische Ergebnisse aus fünf Jahren ökonomischer Begleitforschung zum Extensivierungsprogramm. Arbeitsbericht 1/97, Institut für Betriebswirtschaft der Bundesforschungsanstalt für Landwirtschaft, Braunschweig

NiEBERG H (2001) Unterschiede zwischen erfolgreichen und weniger erfolgreichen Ökobetrieben in Deutschland. Agrarwirtschaft, 50. Jg., Heft 7, S. 428-432

Nieberg H, Offermann F, Zander K (2007) Organic Farms in a Changing Policy Environment: Impacts of Support Payments, EU-Enlargement and Luxembourg Reform, Organic Farming in Europe: Economics and Policy, Volume 13

NUTI I (2003) Bureau Lait, Paris (Gesprächsnotiz); über Gerhard Becker, BMVEL, Bonn

Oerthel F, Tuschl S (1995) Statistische Datenanalyse mit dem Programmpaket SAS, Universität Passau, Lehrstuhl für Statistik, Passau (Oldenbourg Verlag GmbH, München)

OfFermann F, KLeinhanß W, HÜtTel S, KÜPKER B (2005) Assessing the 2003 CAP reform impacts on German agriculture using the farm group model FARMIS. In: Arfini, F. (Hrsg.): Modelling Agricultural Policies. State of the Art. Parma, Italien, S. 546-564

Offermann F, Nieberg H (2000) Economic Performance of Organic Farms in Europe. Organic Farming in Europe: Economics and Policy, Vol. 5, Hohenheim

OfFERMANN F, NiEBERG H (2001) Wirtschaftliche Situation ökologischer Betriebe in ausgewählten Ländern Europas: Stand, Entwicklung und wichtige Einflussfaktoren. In: Agrarwirtschaft, 50. Jg., Heft 7

Perrot C, Fraysse JL (2002) Diversité des exploitations d'élevage de ruminants: principaux facteurs et éléments de quantification à partir du recensement agricole 2000. Institut de l’Elevage et Ministère de l’Agriculture, de l'alimentation, de la pêche et des affaires rurales, Paris

Poppe K-J, Povellato A, Krijgsman K (2003) PACIOLI 10, European farmers and the growing of data, Agricultual Economics Research Institute (LEI), The Hague

RAвовANK (1998): The world dairy market.

REFARDT M, SPILS AD WILKEN A (1999) Jahresabschlussanalyse in der Landwirtschaft. 3. Auflage, Schriftenreihe des Hauptverbandes der landwirtschaftlichen Buchstellen und Sachverständigen e. V. (HLBS), Heft 100

ReIL A (2004) Der Einsatz der Betriebszweigabrechnung in interregionalen und internationalen Betriebsvergleichen. Dissertation

REIL A et al. (2000) EDF-Analysis 2000. Bundesforschungsanstalt für Landwirtschaft, Braunschweig

REIL A et al. (2001) EDF-Analysis 2001. Bundesforschungsanstalt für Landwirtschaft, Braunschweig 
Reil A, Hemme T, Seifert K (2000) EDF-Analysis 2000: Production Cost Comparison, Farm Development Survey, Single Farm Database (EDF - European Dairy Farmers) (Hrsg.) Global farm GbR, Braunschweig

Reil A, Hemme T, Seifert K (2001) EDF-Analysis 2001: Production Cost Comparison, Farm Development Survey, Single Farm Database (EDF - European Dairy Farmers) (Hrsg.) Global farm GbR, Braunschweig

REINHARD S (1999) Econometric analysis of economic and environmental efficiency of Dutch dairy farms. Phd Thesis. Wageningen Agricultural University. The Hague

Richardson JW, NixON CJ (1986) Description of FLIPSIM V: A General Firm Level Policy Simulation Model. Agricultural \& Food Policy Center, Department of Agricultural Economics, Texas Agricultural Experiment Station, USA

SAlamon P (2001) Der Markt für Milch. Agrarwirtschaft 50 (1), S. 40-54

SALAMON P (2002) Proposal for a thematic session at the EAAE Congress. Exploring diversity in the European Agri-Food System. 28-31, August 2002, Zaragoza, Spain. Theme: Modelling the phasing out of milk quotas - methodological problems and solutions. In: Congress of the European Association of Agricultural Economists, 28-31, August 2002, Zaragoza, Spain. Exploring diversity in the European agri-food system

SALAMON P et al. (2002) Modelling the Phasing Out of Milk-Quotas in Europe - An Overview. Presented on the 10. EAAE-Conference, August 28-31, Zaragoza, Spain. Institut für Marktanalyse und Institut für Betriebswirtschaft, Agrarstruktur und ländliche Räume der Bundesforschungsanstalt für Landwirtschaft, Braunschweig

SCHLEEF K-H (1999) Auswirkungen von Stickstoffminderungspolitiken. Schriftenreihe des Bundesministeriums für Ernährung, Landwirtschaft und Forsten, Reihe A: Angewandte Wissenschaft, Heft 482, Dissertation

SCHRIFTEN DER GESELLSCHAFT FÜR WIRTSCHAFTS- UND SOZIALWISSENSCHAFTEN DES LANDBAUS E.V. (1984) Einkommen in der Landwirtschaft - Entstehung, Verteilung, Verwendung und Beeinflussung, Band 21, 1. Auflage 1984, S. 144-145

SCHRIFTEN DER GESELlSCHAFT FÜR WIRTSCHAFTS- UND SOZIALWISSENSCHAFTEN DES LANDBAUS E. V. (1984) Einkommen in der Landwirtschaft - Entstehung, Verteilung, Verwendung und Beeinflussung. Band 21, 1. Auflage, S. 148-151

SCHRIFTEN DER GESELlSCHAFT FÜR WIRTSCHAFTS- UND SOZIALWISSENSCHAFTEN DES LANDBAUES E. V. (1984) Einkommen in der Landwirtschaft - Entstehung, Verteilung, Verwendung und Beeinflussung. Band 21, 1. Auflage, S. 225

SCHRIFTEN DER GESELLSCHAFT FÜR WIRTSCHAFTS- UND SOZIALWISSENSCHAFTEN DES LANDBAUES E. V. (1984) Einkommen in der Landwirtschaft - Entstehung, Verteilung, Verwendung und Beeinflussung. Band 21, 1. Auflage, S. 232 
SCHRIFTENREIHE DES BUNDESMINISTERIUMS FÜR ERNÄHRUNG, LANDWIRTSCHAFT UND FORSTEN (1995) Klimaforschung im Geschäftsbereich des BML, Heft 442, S. 139-150, Landwirtschaftsverlag GmbH, Münster

SCHRIFTENREIHE DES HAUPTVERBANDES DER LANDWIRTSCHAFTLICHEN BUCHSTELLEN UND SACHVERSTÄNDIGEN (HLBS) (1995) Bewertung in der landwirtschaftlichen Buchführung. Heft 88, 4. Auflage, Verlag Pflug und Feder, St. Augustin

SCHUlze PALS L (1994) Ökonomische Analyse der Umstellung auf ökologischen Landbau. Schriftenreihe des Bundesministeriums für Ernährung, Landwirtschaft und Forsten, Reihe A: Angewandte Wissenschaft, Heft 436, S. 85-92, Dissertation

SPÄTH H (1983) Cluster-Formation und -Analyse: Theorie, FORTRAN-Programme und Beispiele. Universität Oldenburg

Statistisches Bundesamt Wiesbaden (1979) Methodische Grundlagen der Agrarberichterstattung, Land- und Forstwirtschaft, Fischerei, Fachserie 3, Reihe 2, S. 1

Statistisches Bundesamt Wiesbaden (1997) Land- und Forstwirtschaft, Fischerei, Fachserie 3, Reihe 2.1.3, Viehhaltung der Betriebe (Ergebnisse der Agrarberichterstattung), Wiesbaden, S. 6-9

Statistisches Bundesamt Wiesbaden (1999) Methodische Grundlagen der Strukturerhebungen in land- und forstwirtschaftlichen Betrieben 1999, Agrarstrukturerhebung/Landwirtschaftszählung 1999, Land- und Forstwirtschaft, Fischerei, Fachserie 3, Reihe 2, S. 2

STEINHAUSEN D, LANGER K (1977) Clusteranalyse - Einführung in Methoden und Verfahren der automatischen Klassifikation. Berlin/New York

Thiele und Brodersen 1997) Anwendung der nicht-parametrischen Data Envelopment Analysis

TietJen A, LANGBEHN C (2000) Bestimmungsfaktoren der Wettbewerbslage von Milchviehbetrieben - eine empirische Analyse. Schriften der Gesellschaft für Wirtschafts- und Sozialwissenschaften des Landbaues e. V., Bd. 36, S. 133-141

Tonini A (2007) Agriculture and Dairy in Eastern Europe after Transition focused on Poland and Hungary, Dissertation, Universität Wageningen, 09.02.2007

USDA Foreign Agricultural Service (2006) GAIN Report, Nr. E36134, EU-25 Dairy and Products Annual 2006, Global Agricultural Information Network, Brüssel

VAN TONGEREN F (2002) Forward-looking analysis of reforms of the EU dairy policy. Paper presented on the 10. EAAE-Conference, August 28-31, Zaragoza, Spain

VAn Tongeren F, VAn MeIJl H (1999) Review of applied models of international trade in agriculture and related resource and environmental modelling, FAIR6 CT 984148 Interim Report No. 1, Report 5.99.11, Agricultural Economics Research Institute (LEI), The Hague, Niederlande 
VARD T (1995) Kosten für die Milcherzeugung in der Europäischen Union 1990/911992/93, Kommission der Europäischen Gemeinschaften, Generaldirektion Landwirtschaft VI/A-3, Analyse der Lage der landwirtschaftlichen Betriebe, Brüssel

VARD T (2000) AIDS2K: A model to estimate EU direct payments in FADN (Base year and Agenda 2000) - Documentation. European Commission, Agriculture Directorate - General (DG6), Brüssel

VARD T (2001) Costs of production for milk in the European Union period 1989/90 1998/99 (revised methodology). European Commission, Directorate-General VI Agriculture, Agri-A.3 Analysis of the situation of agricultural holdings, Brüssel

Verordnung (EWG) Nr. 1859/82 der Kommission vom 12.07.1982 über die Auswahl der Buchführungsbetriebe zum Zweck der Feststellung der Einkommen in den landwirtschaftlichen Betrieben. AB1. Nr. L 205 vom 13.07.1982

Verordnung (EWG) Nr. 2237/77 der Kommission vom 23.9.1977 über den zur Feststellung der Einkommen in den landwirtschaftlichen Betrieben zu benutzenden Betriebsbogen. AB1. Nr. L 263 vom 17.10.1977

Verordnung Nr. 79/65/EWG des Rates vom 15.6.1965 zur Bildung eines Informationsnetzes landwirtschaftlicher Buchführungen über die Einkommenslage und die betriebswirtschaftlichen Verhältnisse landwirtschaftlicher Betriebe in der EWG. AB1. Nr. 109 vom 23.6.1965

WRIGHT I et al. (2003) ELPEN-System, CD-ROM

ZEDDIES J (1991) Wettbewerbsfähigkeit aus Sicht landwirtschaftlicher Betriebe - Situationsbeschreibung und Auswirkungen in ausgewählten Harmonisierungsbereichen. Schriften der Gesellschaft für Wirtschafts- und Sozialwissenschaften des Landbaues e. V., Bd. 27, S. 75-87

ZedDIEs J (2003) Modellgestützte Politikberatung in der Agrar- und Agrarumweltpolitik, Institut für Landwirtschaftliche Betriebslehre der Universität Hohenheim, Stuttgart

Zentrale Markt- und Preisberichtstelle (ZMP) (1999) ZMP-Milch 1999. Bonn

Zentrale MarkT- und Preisberichtstelle (ZMP) (2000) Marktbilanz Getreide, Ölsaaten, Futtermittel 2000; Rainer Stratmann, Bonn

Zentrale MarkT- und Preisberichtstelle (ZMP) (2001) Marktbilanz Getreide, Ölsaaten, Futtermittel 2001, Rainer Stratmann, Bonn

Zentrale MarkT- Und Preisberichtstelle (ZMP) (2002; 2006) Marktbilanz Milch. Deutschland, Europäische Union, Weltmärkte, Bonn

Zentrale Markt- und Preisberichtstelle (ZMP) (2003) Marktbilanz Milch 2003

Zentrale MarkT- Und Preisberichtstelle (ZMP) (2003) Marktbilanz Vieh und Fleisch 2003 


\section{Anhang}




\section{Anhang zu Kapitel 2}

Tabelle A2.1: Milchquoten: Gesamtreferenzmenge je Mitgliedstaat (1999/2000)

\begin{tabular}{|c|c|c|c|c|}
\hline & \multicolumn{2}{|c|}{ Gesamtquote } & \multicolumn{2}{|c|}{ Direktvermarktungsquote } \\
\hline & $\mathbf{t}$ & $\%$ & $\mathbf{t}$ & $\%$ \\
\hline Belgien & 3.140 .696 & 2,7 & 169.735 & 10,5 \\
\hline Dänemark & 4.454 .640 & 3,8 & 708 & 0,04 \\
\hline Deutschland & 27.767 .036 & 24,0 & 97.780 & 6,0 \\
\hline Griechenland & 629.817 & 0,5 & 696 & 0,04 \\
\hline Spanien & 5.457 .564 & 4,7 & 109.386 & 6,8 \\
\hline Frankreich & 23.793.932 & 20,5 & 441.866 & 27,3 \\
\hline Irland & 5.236 .575 & 4,5 & 9.189 & 0,6 \\
\hline Italien & 9.698 .399 & 8,4 & 231.661 & 14,3 \\
\hline Luxemburg & 268.098 & 0,2 & 951 & 0,1 \\
\hline Niederlande & 10.991 .900 & 9,5 & 82.792 & 5,1 \\
\hline Österreich & 2.543 .979 & 2,2 & 205.422 & 12,7 \\
\hline Portugal & 1.835 .461 & 1,6 & 37.000 & 2,3 \\
\hline Finnland & 2.394 .528 & 2,1 & 10.000 & 0,6 \\
\hline Schweden & 3.300 .000 & 2,8 & 3.000 & 0,2 \\
\hline Vereinigtes Königreich & 14.373 .969 & 12,4 & 216.078 & 13,4 \\
\hline Summe & 115.886 .594 & 100 & 1.616 .264 & 100 \\
\hline
\end{tabular}

1) Die Milchquoten gelten vom 1 . April bis 31. März jeden Jahres.

Quelle: Europäische Kommission, Generaldirektion Landwirtschaft (2003): Reform der GAP: Milch und Milcherzeugnisse; http://europa.eu.int/comm/agriculture. 
Tabelle A2.2: $\quad$ Angebot und Verbrauch von Käse in der EU-25

\begin{tabular}{|c|c|c|c|}
\hline \multirow[t]{2}{*}{$\begin{array}{l}\text { Land: } \\
\text { Produkt: }\end{array}$} & \multicolumn{3}{|c|}{$\begin{array}{ll} & \text { EU-25 } \\
\text { Käse } & (1.000 \text { Mio. t })\end{array}$} \\
\hline & 2005 & 2006 & 2007 \\
\hline Kalender/Jahresbeginn & $01 / 2005$ & $01 / 2006$ & $01 / 2007$ \\
\hline Anfangsbestand & 0 & 0 & 0 \\
\hline Produktion & 6,48 & 6,58 & 6,70 \\
\hline Extra EU-25 Importe & 95 & 101 & 100 \\
\hline Gesamtes Angebot & 6,575 & 6,681 & 6,800 \\
\hline Extra EU-25 Exporte & 492 & 529 & 550 \\
\hline Inländischer Verbrauch & 6,083 & 6,152 & 6,250 \\
\hline Andere Nutzung, Verluste & 0 & 0 & 0 \\
\hline Gesamter inländischer Verbrauch & 6,083 & 6,152 & 6,250 \\
\hline Endbestand & 0 & 0 & 0 \\
\hline Gesamter Absatz & 6,575 & 6,681 & 6,800 \\
\hline
\end{tabular}

Quelle: USDA foreign Agricultural Service, GAIN Report (2006).

Tabelle A2.3: Angebot und Verbrauch von Butter in der EU-25

\begin{tabular}{lrrr}
\hline $\begin{array}{l}\text { Land: } \\
\text { Produkt: }\end{array}$ & \multicolumn{3}{c}{ EU-25 } \\
& $\mathbf{2 0 0 5}$ & $\mathbf{2 0 0 6}$ & $\mathbf{2 0 0 7}$ \\
\hline & $01 / 2005$ & $01 / 2006$ & $01 / 2007$ \\
Kalender/Jahresbeginn & & & \\
& 232 & 185 & 122 \\
Anfangsbestand & 2,140 & 2,055 & 2,055 \\
Produktion & 82 & 84 & 85 \\
Extra EU-25 Importe & 2,454 & 2,322 & 2,262 \\
Gesamtes Angebot & 345 & 254 & 260 \\
& 1,924 & 1,948 & 1,952 \\
Extra EU-25 Exporte & 0 & 0 & 0 \\
Inländischer Verbrauch & 1,924 & 1,948 & 1,952 \\
Andere Nutzung, Verluste & & & 50 \\
Gesamter inländischer Verbrauch & 185 & 122 & 2,262 \\
& 2,454 & 2,322 & \\
$\quad$ Endbestand & & & \\
Gesamter Absatz & & & \\
\hline
\end{tabular}

Quelle: USDA foreign Agricultural Service, GAIN Report (2006). 
Tabelle A2.4: Angebot und Verbrauch von Vollmilchpulver in der EU-25

\begin{tabular}{lrrr}
\hline & $\mathbf{2 0 0 5}$ & $\mathbf{2 0 0 6}$ & $\mathbf{2 0 0 7}$ \\
\hline Kalender/Jahresbeginn & $01 / 2005$ & $01 / 2006$ & $01 / 2007$ \\
Beginning Stocks & 0 & 0 & 0 \\
Production & 858 & 810 & 805 \\
Extra EU25 ImportE & 2 & 2 & 2 \\
Gesamtes Angebot & 860 & 812 & 807 \\
& & & 430 \\
Extra EU-25 Exporte & 493 & 430 & 377 \\
Inländischer Verbrauch & 367 & 382 & 0 \\
Andere Nutzung, Verluste & 0 & 0 & 377 \\
Gesamter inländischer Verbrauch & 367 & 382 & 0 \\
Endbestand & & & 807 \\
Gesamter Absatz & 860 & 812 & 0 \\
\hline
\end{tabular}

Quelle: USDA foreign Agricultural Service, GAIN Report (2006).

Tabelle A2.5: Angebot und Verbrauch von Magermilchpulver in der EU-25

\begin{tabular}{lrrr}
\hline $\begin{array}{l}\text { Land: } \\
\text { Produkt: }\end{array}$ & \multicolumn{3}{c}{$\begin{array}{c}\text { EU-25 } \\
\text { Magermilchpulver (1.000 Mio. t) }\end{array}$} \\
& $\mathbf{2 0 0 5}$ & $\mathbf{2 0 0 6}$ & $\mathbf{2 0 0 7}$ \\
\hline Kalender/Jahresbeginn & $01 / 2005$ & $01 / 2006$ & $01 / 2007$ \\
Anfangsbestand & 77 & 8 & 0 \\
Produktion & 1,065 & 975 & 975 \\
Extra EU-25 Importe & 7 & 19 & 20 \\
Gesamtes Angebot & 1,149 & 1,002 & 995 \\
& 194 & & 100 \\
Extra EU-25 Exporte & 947 & 914 & 895 \\
Inländischer Verbrauch & 0 & 0 & 0 \\
Andere Nutzung, Verluste & 947 & 914 & 095 \\
Gesamter inländischer Verbrauch & & & 995 \\
& 8 & 0 & \\
Endbestand & 1,149 & 1,002 & \\
Gesamter Absatz & & & \\
\hline
\end{tabular}

Quelle: USDA foreign Agricultural Service, GAIN Report (2006). 


\section{Anhang zu Kapitel 3}

Abbildung A3.1: Modelle im Modellverbund der Bundesforschungsanstalt für Landwirtschaft (FAL)

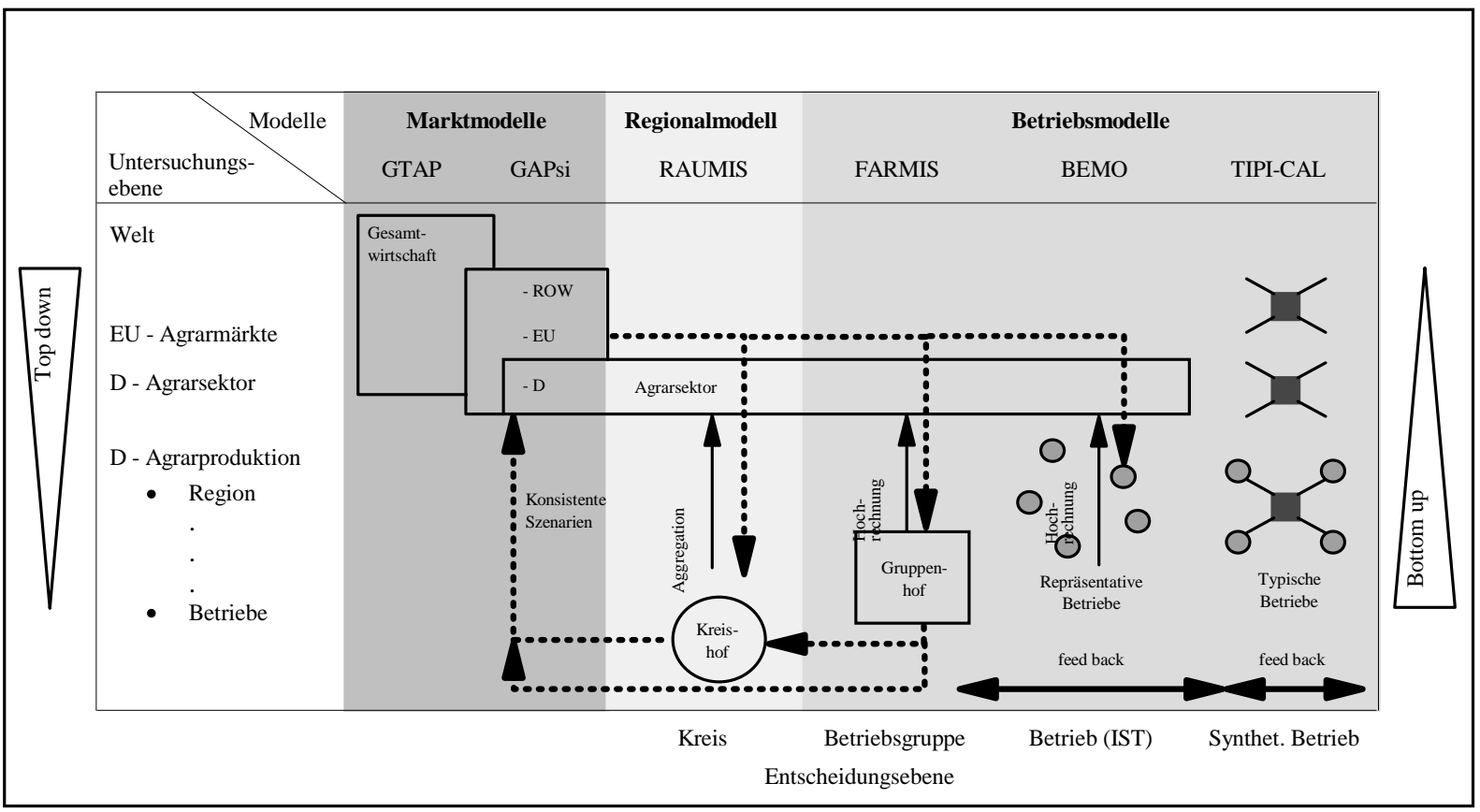

Quelle: Kleinhanß (2000). 
Abbildung A3.2: Aufbau des Betriebsgruppenmodells FARMIS

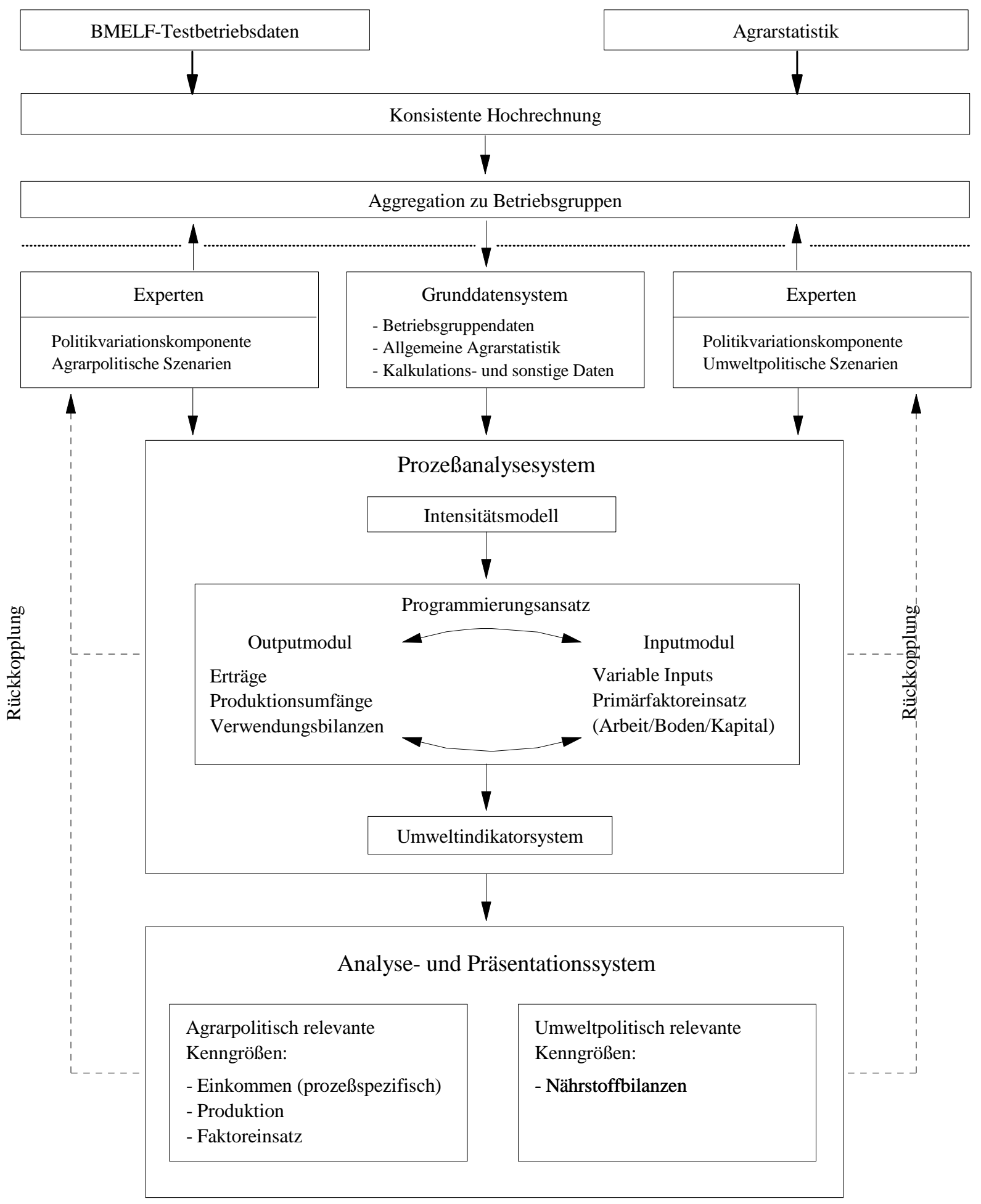

Quelle: Schleef (1990). 


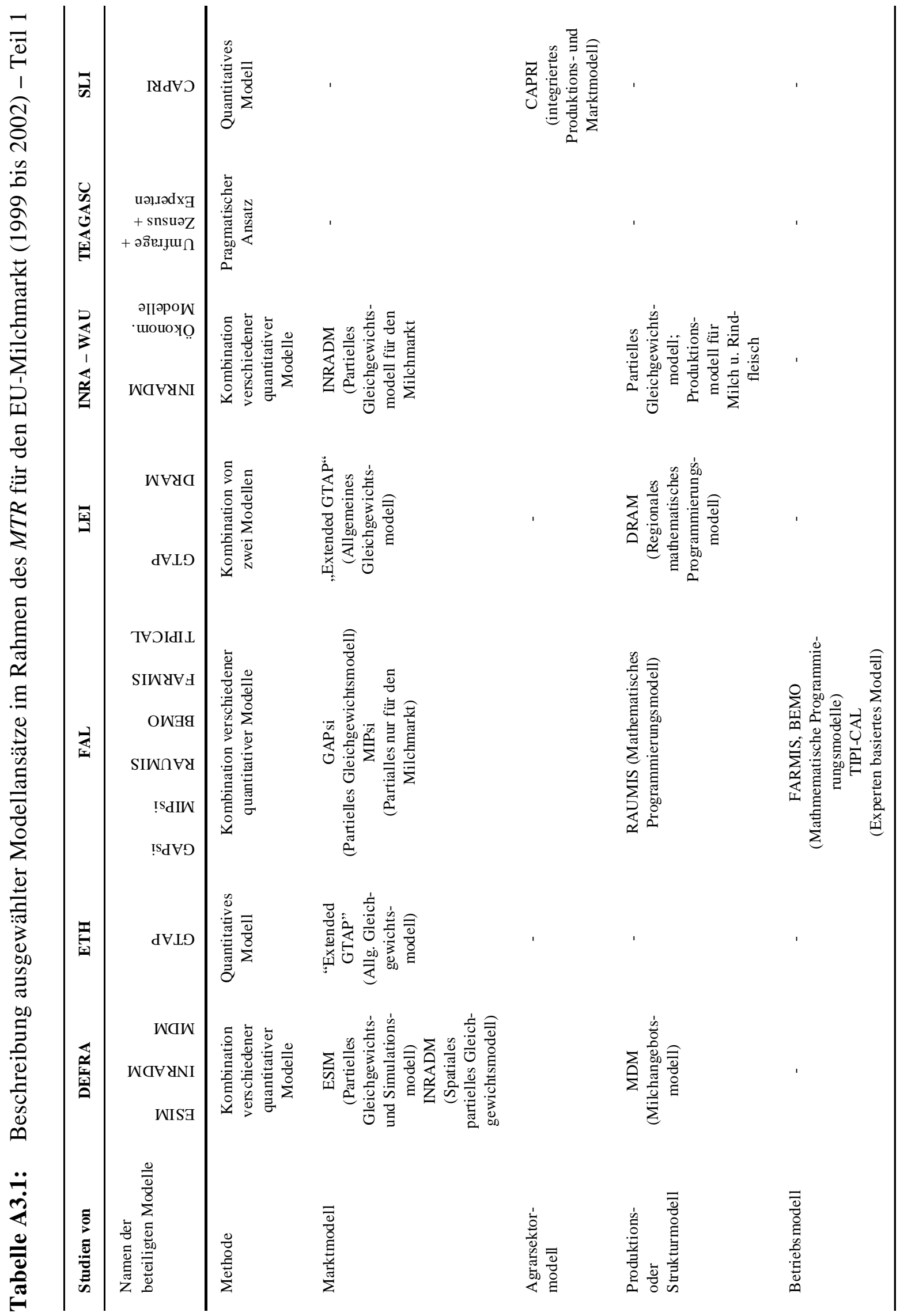




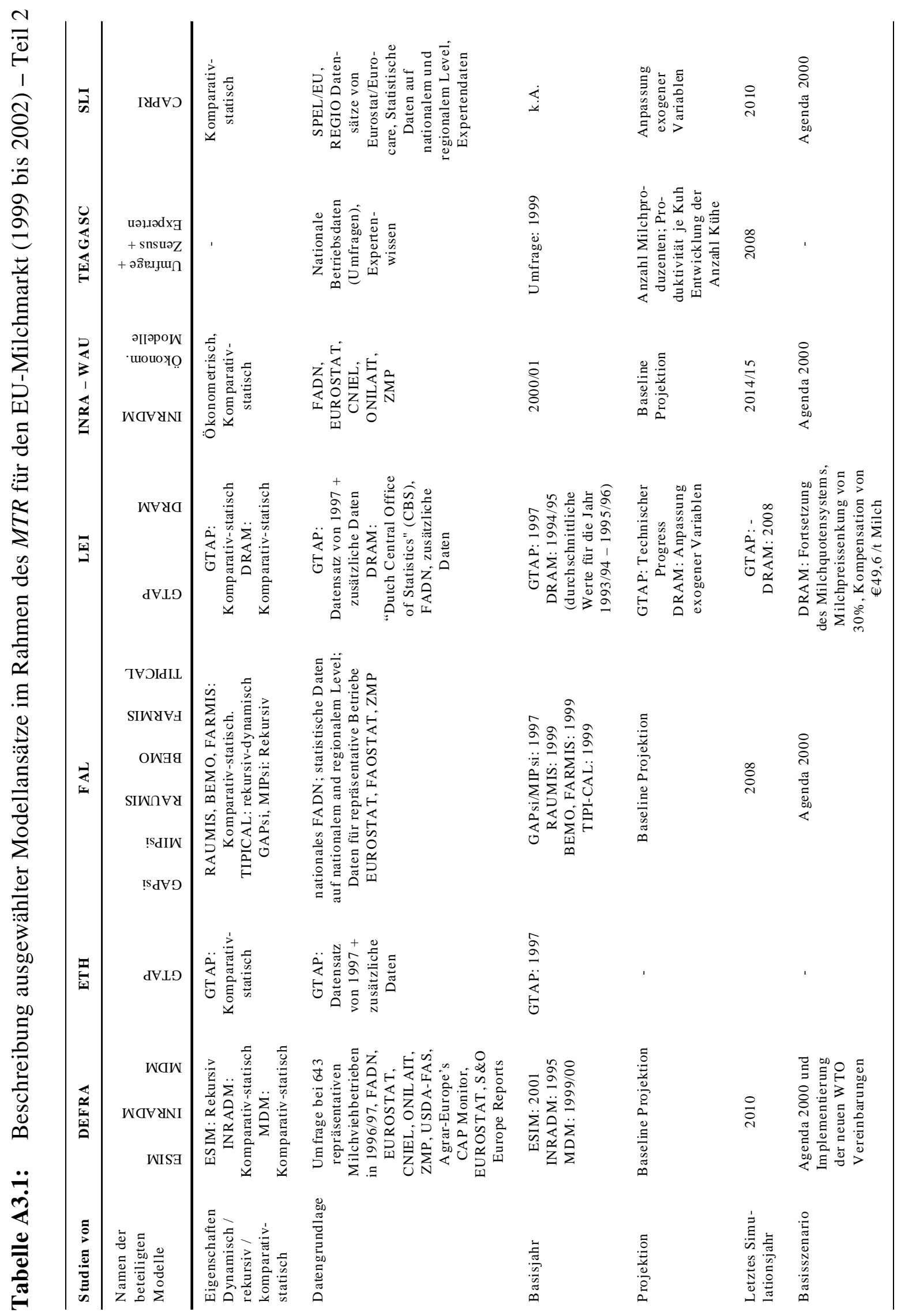




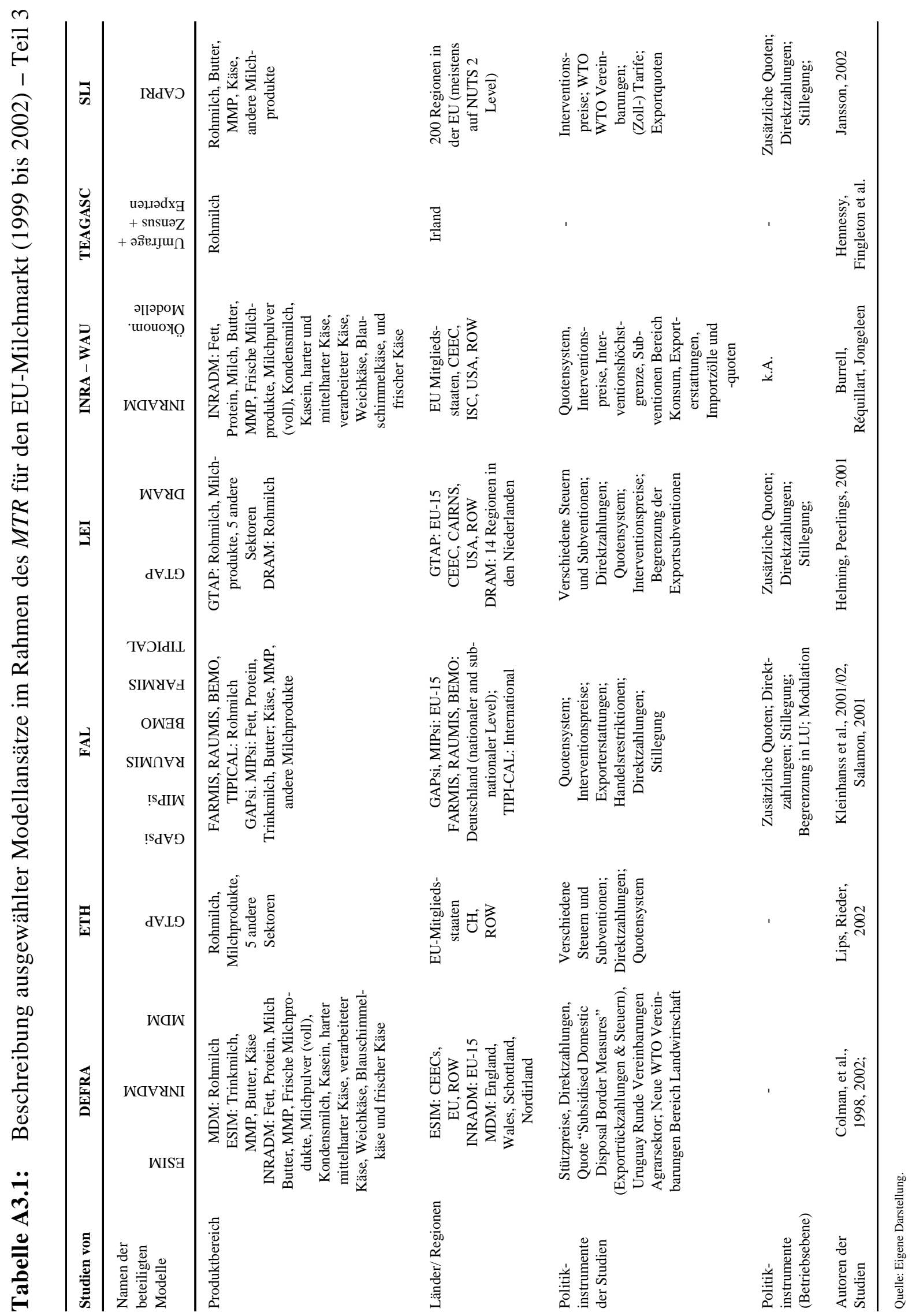




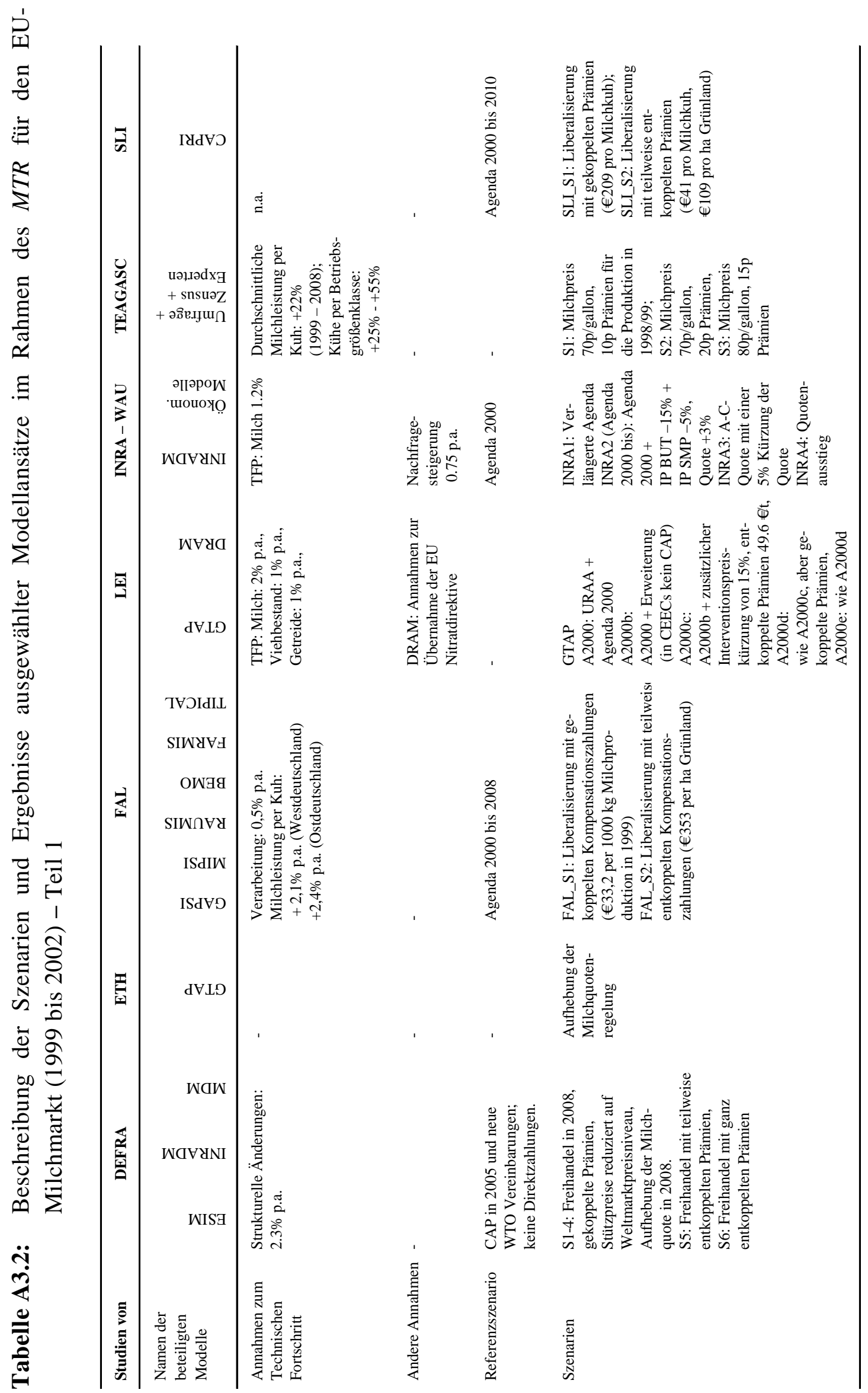




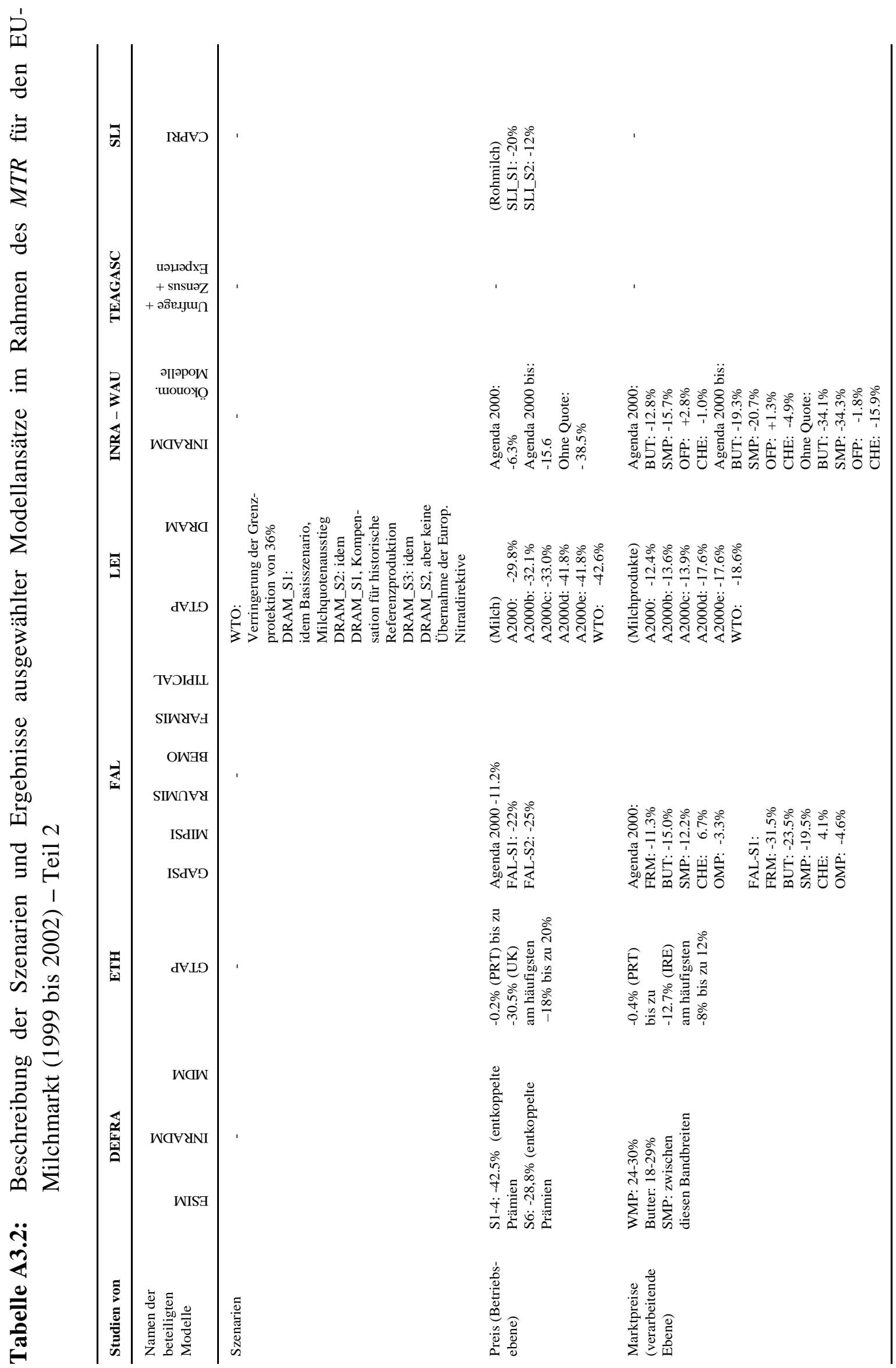




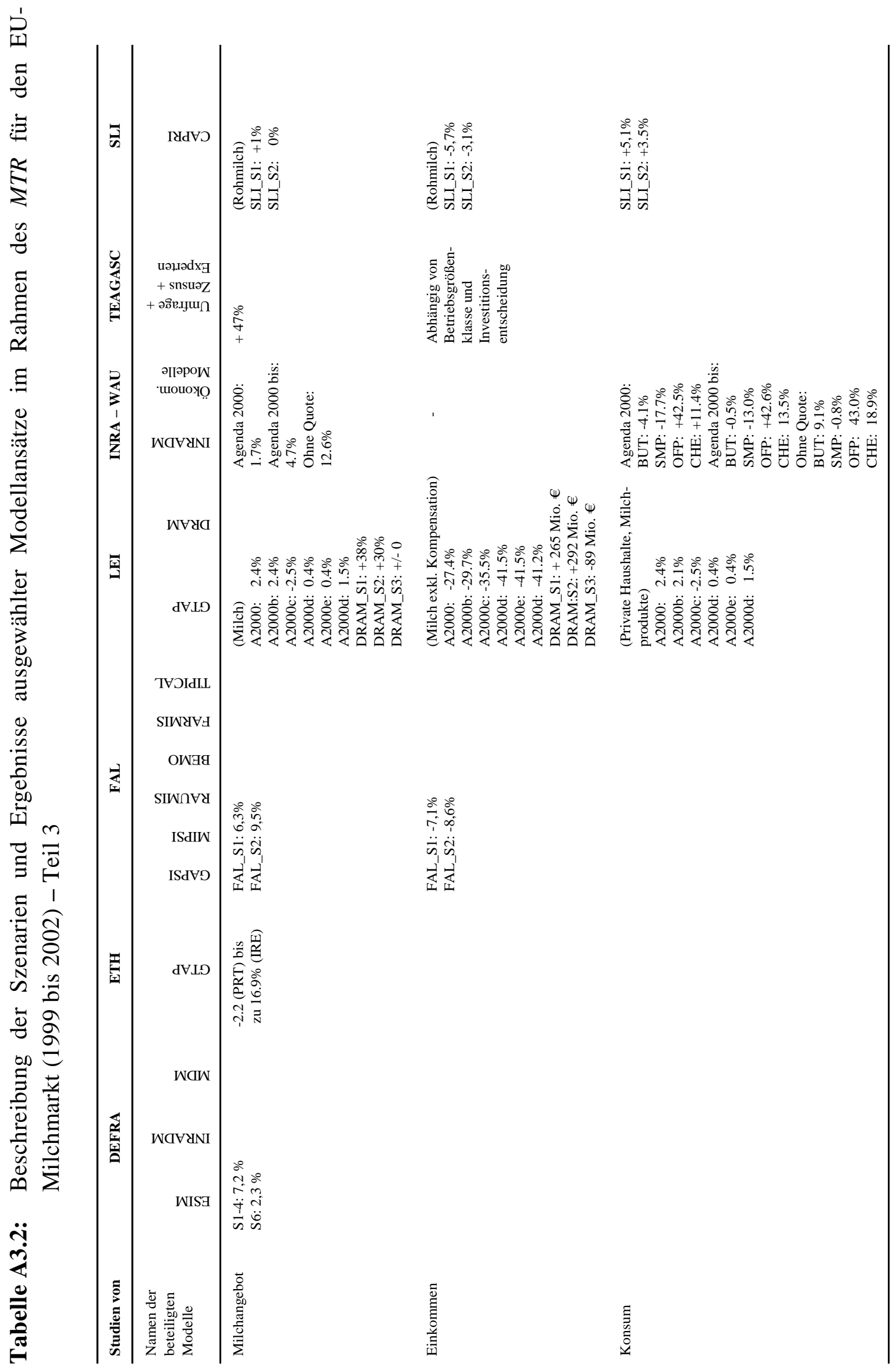




\section{Anhang zu Kapitel 4}

Tabelle A4.1: $\quad$ Festlegung unterschiedlicher Auswahlschwellen (in ESU) in den verschiedenen EU-Mitgliedsländern zur Auswahl der Betriebe für die FADN-Stichprobe (von der Kommission angewandt für das Jahr 1997)

\begin{tabular}{lc}
\hline EU Mitgliedstaat (1997) & Größenschwellen (in ESU) \\
\hline Belgien & 12 \\
Dänemark & 8 \\
Deutschland & 8 \\
Griechenland & 2 \\
Spanien & 2 \\
Frankreich & 8 \\
Irland & 2 \\
Italien & 2 \\
Luxemburg & 8 \\
Niederlande & 16 \\
Österreich & 8 \\
Portugal & 1 \\
Finnland & 8 \\
Schweden & 8 \\
Vereinigtes Königreich (Northern Ireland) & 4 \\
Vereinigtes Königreich & 8 \\
\hline
\end{tabular}

Quelle: http://europa.eu.int/comm/agriculture/rica/methodology1_de.cfm 


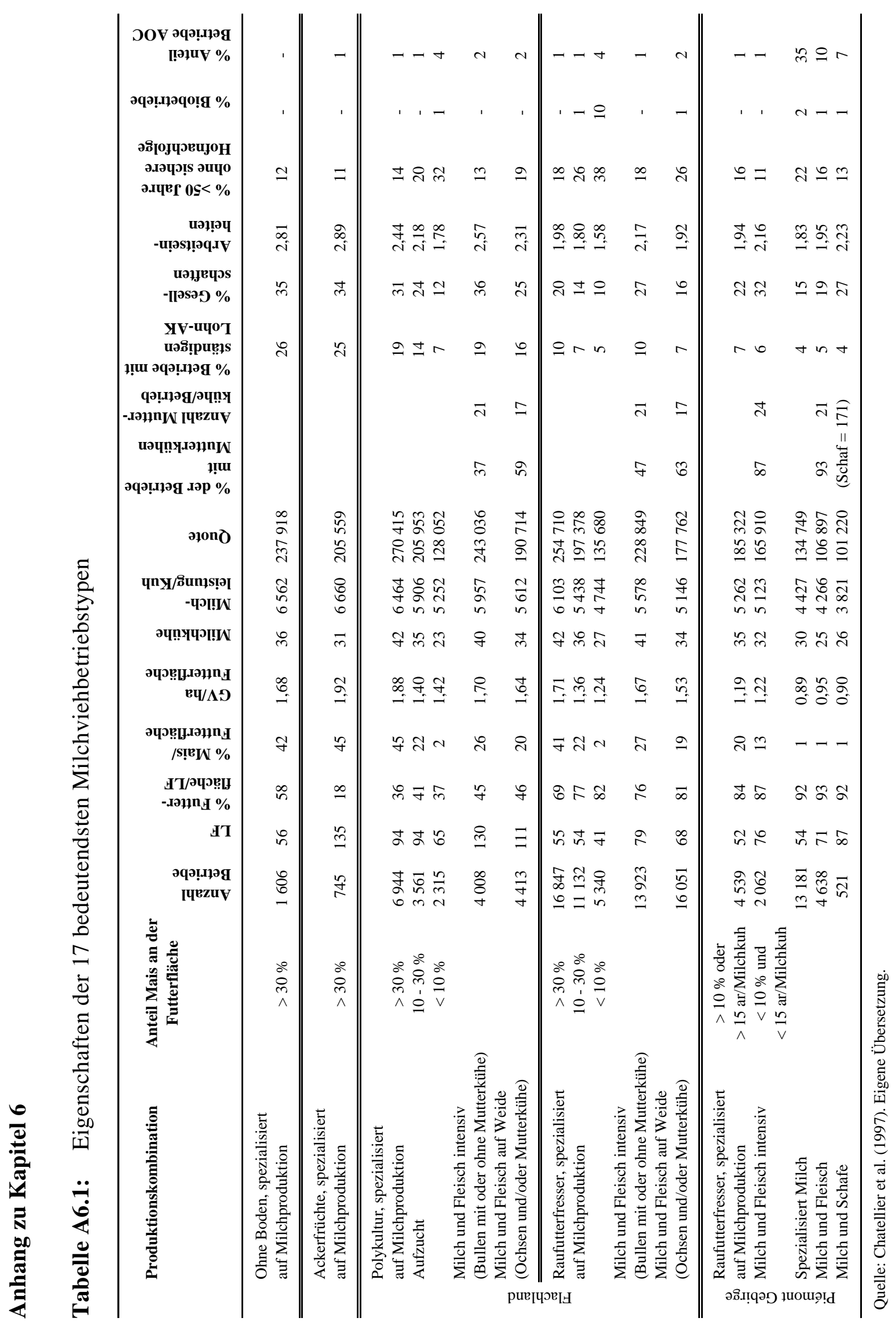




\section{Zu Tabelle A6.1: $\quad$ Vorgehen bei der Schätzung der Produktionskosten}

Die Berechnung der Produktionskosten erfolgt auf der Ebene jedes Einzelbetriebs. Im Folgenden wird aufgezeigt, wie der FADN-Datensatz anzupassen ist, um ihn zur statistischen Einordnung typischer Betriebe verfügbar zu machen.

Die Produktionskosten im FADN werden soweit wie möglich aus den Aufwandspositionen der Gewinn-und-Verlust-Rechnung (GuV) ermittelt. ${ }^{1}$ Die Produktionskosten sind im FADN größtenteils nur aggregiert auf Ebene des Betriebes oder verschiedener Produktionsbereiche erfasst. Daher werden die erfassten Kosten aus dem FADN für die vorliegende Analyse mit Hilfe verschiedener Allokationsfaktoren soweit wie möglich dem Betriebszweig Milchproduktion zugeteilt. Zur Berechnung der kalkulatorischen Kosten werden die regional üblichen Kostensätze für Boden, Arbeit und Kapital herangezogen.

Im Folgenden wird auf die Vollkostenrechnung und auf die Behandlung der Nebenerlöse eingegangen. Es folgt die Erläuterung einzelner Kostenansätze und die Aufteilung der gesamten Kostengrößen auf den Betriebszweig Milch. Abschließend wird auf die Datenkontrollen Bezug genommen, die Voraussetzung aller Berechnungen ist.

\section{Vollkostenrechung und Abzug der Nebenerlöse}

Die Kostenanalyse erfolgt im Modell TIPI-CAL im Rahmen des IFCN auf Basis der Vollkostenrechnung. Berechnet werden zunächst die Vollkosten, die auch die Kosten für die Nebenerzeugnisse umfassen. Die bedeutendsten Nebenerzeugnisse sind Altkühe, Kälber und Färsen. ${ }^{2}$ Da die Produktionskosten der verschiedenen Nebenerzeugnisse auf Basis der vorhandenen Datengrundlage nicht einzeln zu berechnen sind, wird bei der Berechnung der Milchproduktionskosten vereinfachend davon ausgegangen, dass die Erlöse der Nebenerzeugnisse exakt den Kosten ihrer Erstellung entsprechen. ${ }^{3}$ Um die Kosten zu ermitteln, die ausschließlich auf die Milcherzeugung entfallen, werden die Nebenerlöse (für Altkühe, Kälber und Färsen) von den Gesamtkosten abgezogen (vgl. IsERMEYER, 1988; Hemme et al., 1997; Hemme, 2000). Die auf diese Weise ermittelten Vollkosten können direkt dem Milchpreis gegenübergestellt werden. Kritisch ist diese Methode dann zu beurteilen, wenn der Erlös der Nebenprodukte wesentlich höher als die Kosten ihrer Erzeugung ausfallen. Dies kann beispielsweise eintreten im Fall hoher Direktzahlungen (Beispiel Finnland), hoher Rindfleischpreise (Beispiel bayerische Fleckviehbetriebe), spezieller

1 Die Produktionskosten werden pro Liter Milch ausgewiesen und auf 4 \% Fett korrigiert (vgl. HoLZNER (2003, S. 109).

2 Weitere Nebenprodukte: Verkaufter Wirtschaftsdünger (Gülle, Mist, Jauche).

3 Eine ausführliche Darstellung dieser Vorgehensweise, des sogenannten Nettokonzeptes, ist nachzulesen bei IsERmeYer (1988), Hemme et al. (1997), Hemme (2000, S. 76), Holzner (2003, S. 119 ff.). 
Milch- und Fleischrinderrassen (Beispiel indische Büffelbetriebe) oder dem Verkauf von Wirtschaftsdünger. Diese Besonderheiten können nicht berücksichtigt werden. Bei dem vorhandenen Datenmaterial ist der Schätzfehler, der bei der exakten Berechnung der Kosten für die Nebenerlöse auftreten kann, wahrscheinlich höher, als der Fehler, der aufgrund der getroffenen Annahme in einzelnen Fällen möglicherweise entsteht.

Im Folgenden wird auf die Berechnung einzelner FADN-Kostenpositionen eingegangen:

Landkosten: Im FADN liegen für die Untersuchungsbetriebe Hektarangaben über die gesamte landwirtschaftliche Nutzfläche, die Acker- und Grünlandfläche und die Eigentumsfläche (insgesamt sowie für Acker- und Grünland) vor. Die Differenz zwischen der gesamten Fläche (LF, Acker-/ Grünland) und dem Eigenanteil ergibt die jeweils abgeleitete Pachtfläche. Der Pachtaufwand ist im FADN nicht differenziert nach Acker- und Grünland sondern liegt nur für die gesamte landwirtschaftliche Nutzfläche vor. Die gesamtbetrieblichen Pachtzahlungen wurden auf die Acker- und Grünlandpachtfläche bezogen. Beim Auftreten unplausibler Werte wird der IFCN-Pachtsatz herangezogen.

\section{Futterkosten}

Der Wert der betriebseigenen Futtererzeugung wird anhand der spezifischen Kosten für die verschiedenen Futterpflanzen (d. h. gekauftes oder im Betrieb erzeugtes Saatgut und z.B. Pflanzenschutz) unter Berücksichtigung des Flächenumfangs berechnet (vgl. VARD, 1995, 2001 ${ }^{4}$ ). Der Wert der betriebseigenen Futtererzeugung wird ermittelt, indem die spezifischen Kosten addiert und mit ihrem jeweiligen Flächenumfang (Hauptfutterfläche) multipliziert werden. Angaben über zugekauftes Futter liegen nur für den Bereich der Rinderproduktion insgesamt vor und müssen mit Hilfe von Algorithmen dem Milchbereich zugeordnet werden (Methode s. u.).

\section{Remontierungskosten}

Die Umtriebsrate lässt sich mit dem FADN-Datensatz aus dem Jahr 1999/2000 nicht ableiten. Die physischen Angaben zu Viehverkäufen und -zukäufen liegen erst ab dem Jahr 2002 im FADN-Datensatz vor. Der Bestand an Jungrindern bis zu einem Jahr und die Kälbermast sind jedoch nicht nach Geschlecht unterteilt. Die Remontierungskosten lassen sich nur auf Basis der Kosten für den Färsenzukauf schätzen.

Beim einjährigen Datenvergleich wird mit Hilfe der Datensätze aus dem IFCN eine Annahme für die Umtriebsrate getroffen. Die im FADN-Datensatz verzeichneten Kosten für den Zukauf von Milchkühen (Färsen) werden mit der aus dem IFCN abgeleiteten Umtriebsrate multipliziert.

4

„Graskosten“ = angekauftes und selbst erzeugtes Saatgut (VARD, 1995, S. 15-16). 
Im Rahmen der Simulation von FADN-Daten in Kapitel 7.3 wird differenzierter versucht, einen Schätzwert für die Remontierungsrate und -kosten zu ermitteln.

\section{Abschreibungen}

Die Abschreibungen für Maschinen und Gebäude werden im FADN auf den Wiederbeschaffungswert bezogen. Im IFCN hingegen erfolgte zum Zeitpunkt dieser Arbeit die Abschreibung für Maschinen und Gebäude linear - bezogen auf den Anschaffungswert - bis zu einem Residualwert von Null. ${ }^{5}$ Dies kann zur Unterschätzung der Abschreibungen in den IFCN-Betrieben im Vergleich mit den FADN-Betrieben führen. Dieses unterschiedliche Vorgehen wird jedoch als nicht so bedeutsam eingestuft.

\section{Arbeitskosten}

Expertenerfahrungen im Rahmen des EU-Projektes ELPEN - European Livestock Policy Evaluation Network (WRIGHT et al., 2003) - haben gezeigt, dass die Angaben der Betriebsleiter im FADN-Betriebsbogen häufig zur Überschätzung des Arbeitsaufwandes führen. Betriebsleitern fällt es häufig schwer, den Umfang einer AK-Einheit zu schätzen, und in Teilzeit mitarbeitende Familienangehörige werden oft als volle Familienarbeitskrafteinheiten eingestuft.

Dies ist auch häufig dann der Fall, wenn es eine enge Zusammenarbeit zwischen Produktion und Verarbeitung gibt (z. B. in Italien) und die gesamten Kosten der Milchproduktion zugerechnet werden. Daher werden die auf die Milcherzeugung entfallenden Arbeitskosten häufig überbewertet (PERROT et al, 2007). Die hofeigene Verarbeitung ist nicht weit verbreitet und kann in dieser Arbeit deshalb vernachlässigt werden.

In dieser Arbeit werden die Arbeitskrafteinheiten dann korrigiert (siehe Abbildung A6.1), wenn der Arbeitsaufwand außerhalb des definierten Plausibilitätsintervalls (KURATORIUM FÜR TECHNIK UND BAUWESEN IN DER LANDWIRTSCHAFT, verschiedene Jahrgänge) liegt. Darüber hinaus gehende Justierungen, um die Überschätzungen zu reduzieren und um die Arbeitskosten besser abzubilden, waren aufgrund der begrenzten Datenlage nicht möglich. Die tendenzielle Überschätzung des Arbeitsaufwandes ist bei der Interpretation zu berücksichtigen. 


\section{Quotenkosten}

Die Milchquotenkosten sind im FADN-Datensatz in den betrachteten Untersuchungsregionen und Zeiträumen nicht angegeben.

\section{Opportunitätskosten}

Eine Erfassung der betriebsindividuellen Opportunitätskosten für die betriebseigenen Faktoren Arbeit, Boden und Kapital ist in der Praxis schwierig und anhand der vorliegenden anonymisierten FADN-Daten ausgeschlossen, weil die betriebsspezifischen Bedingungen für die Wertansätze nicht bekannt sind (AMERICAN AGRICULTURAL ECONOMIC AssociatION, 2000).

Daher werden ergänzend regionale und betriebstypenspezifische Informationen aus dem IFCN- und EDF-Datensatz herangezogen (EDF, vgl. REIL et al., 2000). Die Opportunitätskosten je Einheit (Hektar, Arbeitseinheit, $1.000 €$ Eigenkapital) werden auf der Stufe der Betriebsgruppen (Mitgliedstaaten, Milchwirtschaftsbetriebe der FADN-Regionen, etc.) geschätzt, und die Beträge für jeden Einzelbetrieb nach Maßgabe der vorhandenen familiären Produktionsfaktoren berechnet. In Anlehnung an die Methoden des IFCN und EDF werden folgende Bewertungsansätze gewählt:

- Zur Bewertung der familieneigenen Arbeit wird die Anzahl der eingesetzten Stunden mit dem regionalen Lohnsatz für eine qualifizierte Arbeitskraft (d. h. für eine AWU) bewertet. Aus dem EDF-Lohnansatz (EDF-ANALYSIS, 2000; REIL et al., 2000) und der Zahl Arbeitsstunden pro AK wird ein Vergleichslohn abgeleitet. Arbeitnehmerbeiträge zu Sozialversicherungen sind eingeschlossen.

- Das gebundene Eigenkapital ohne Bodenvermögen wurde mit 3 \% (nominal) verzinst. $^{6}$

- Die Eigentumsfläche wird mit den durchschnittlich gezahlten Pachtpreisen der Betriebsgruppe und Region bewertet. Liegen diese Pachtpreise aus dem FADN-Datensatz außerhalb des Plausibilitätsbereichs ${ }^{7}$, so werden die Werte mit dem erfragten EDFPachtsatz (EDF-ANALYSIS, 2000; REIL et al., 2000) aus dem Jahr 1999 korrigiert.

Um die für den Gesamtbetrieb bzw. für mehrere Betriebszweige zusammengefassten Kostengrößen ausschließlich auf den Betriebszweig Milch zu beziehen, wird wie folgt vorgegangen.

6 Zur Begründung für diese Vorgehensweise siehe IsERMEYER (1988, S. 108 ff.).

7 Der Plausibilitätsbereich wird mit Hilfe von Expertenbefragungen festgelegt. 


\section{Algorithmen zur Aufteilung der Kosten auf den Betriebszweig Milch}

Die Milchproduktion ist im FADN nicht separat erfasst, weder auf der Einnahmenseite, noch auf der Ausgabenseite. Deshalb wird ein Schätzalgorithmus entwickelt, um die Produktionskosten für die Milch- und Rindfleischproduktion ${ }^{8}$ soweit wie möglich voneinander zu trennen.

Um aus den zusammengefassten Kostenpositionen den Anteil des Betriebszweiges Milch zu generieren, werden die Aufwendungen für die Milcherzeugung in Anlehnung an die IFCN-Methoden anhand folgender Schlüssel aufgerechnet:

- Die Zuteilung der Kosten für Rinder erfolgt nach Maßgabe der Variable „Anteil der Milchkühe am Gesamtbestand der Rinder“. Dieser Zuteilungsschlüssel wird gewählt, weil bei den tierspezifischen Kosten keine Unterscheidung zwischen Milchkühen und sonstigen Rindern getroffen werden kann.

- Die Zuteilung der Arbeit, des Kapitals, der Abschreibung sowie der Gemeinkosten erfolgt nach Maßgabe der Variable „Anteil der Milcherlöse inkl. der Nebenerlöse an den Gesamterlösen“.

- Zuteilung der Flächenkosten sowie der Kosten der Grundfutterproduktion ohne Arbeits- und Maschinenkosten erfolgt nach Maßgabe der Variable „Anteil der Hauptfutterfläche an der landwirtschaftlichen Nutzfläche“ multipliziert mit dem Quotienten „Anteil der Milchkühe am Gesamtbestand der Rinder“.

- Spezialkosten des Betriebszweigs Milch werden vollständig zugeteilt. ${ }^{9}$

Die hier verwendete Schätzmethode entspricht in ihren Grundzügen dem Aufteilungsschlüssel, der bei VARD (1995, 2001) Verwendung fand:

1. Anteil an den gesamten Vieheinheiten: für die spezifischen Kosten (hauptsächlich Futtermittelkosten).

2. Anteil an der gesamten LF: für selbsterzeugtes Futter.

3. Anteil an Gesamterlösen (inkl. Subventionen): für die sonstigen Kosten.

$\mathrm{Zu}$ Veranschaulichung sind die verwendeten Aufteilungsschlüssel nochmals detailliert in Tabelle A6.2 aufgeführt und denen bei VARD verwendeten Allokationsfaktoren gegenübergestellt.

8 Die Produktionskosten anderer raufutterfressender Tiere sind nicht berücksichtigt, da sie bei den einbezogenen spezialisierten Milchviehbetrieben kaum von Bedeutung sind und deswegen vernachlässigt werden können.

9

Die Spezialkosten sonstiger Rinder sind nicht ausgewiesen. 
Tabelle A6.2: $\quad$ Schlüssel für die Aufteilung der Kosten auf die Milcherzeugung

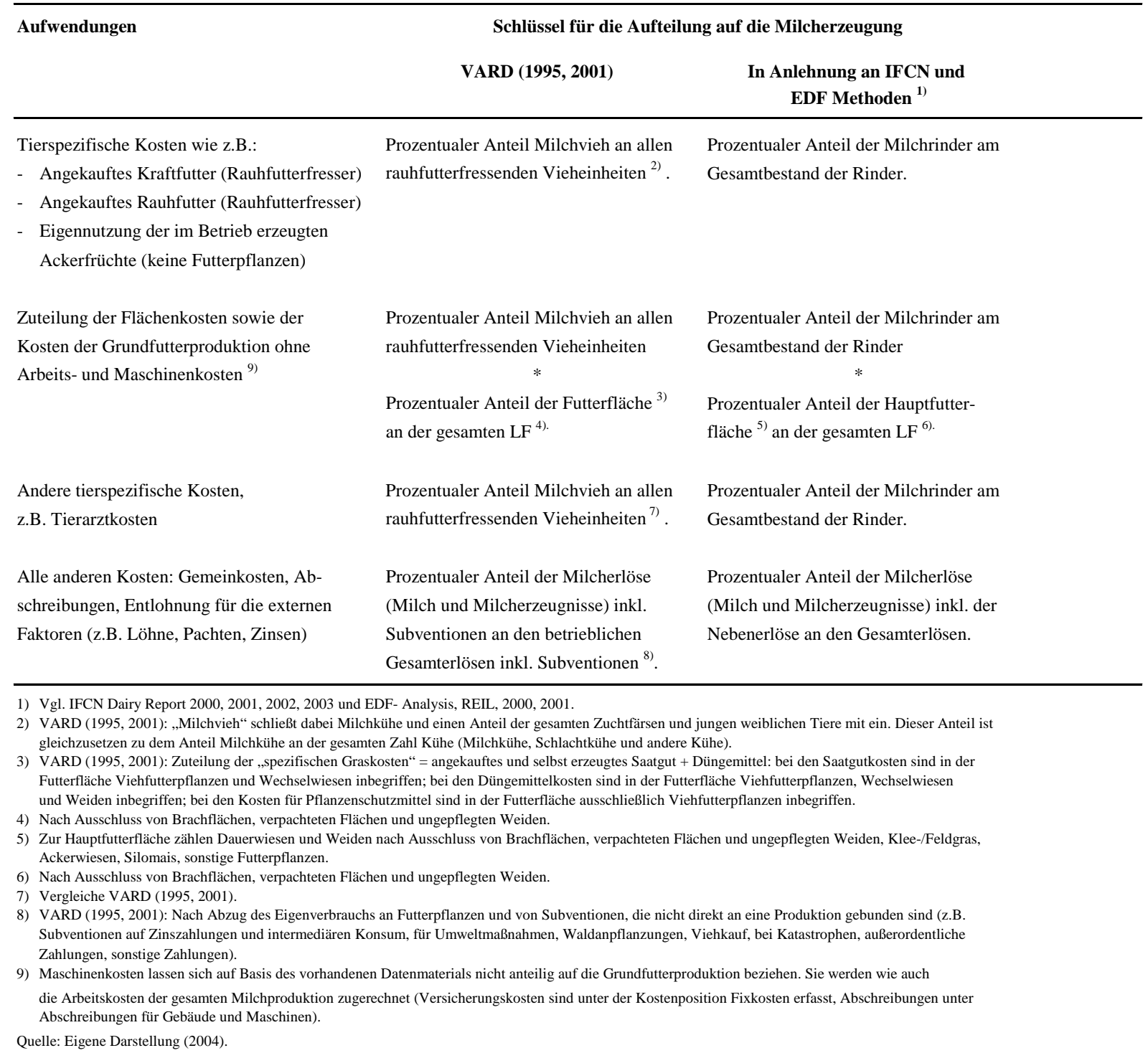

Ein Hauptunterschied zu VARD (1995, 2001) ist die Bezugbasis des verwendeten Allokationsschlüssels „Anteil Milchrinder am Gesamtbestand der Rinder“ bzw. bei VARD „Anteil Milchrinder am Gesamtbestand raufutterfressender Vieheinheiten“. Bei VARD wurde die gesamte tierische Erzeugung einbezogen, während die Kostenallokation in dieser Arbeit nur für den Betriebszweig Milch erfolgt.

Die in dieser Analyse verwendeten Aufteilungsschlüssel für die familieneigenen Faktoren sind in Tabelle A6.3 aufgeführt und denen bei VARD verwendeten Allokationsfaktoren (VARD, 1995) gegenübergestellt.

Auch die verwendeten Bewertungsansätze familiärer Produktionsfaktoren stimmen in ihren Grundzügen mit den Schätzkoeffizienten bei VARD (1995) überein. Unterschiede be- 
stehen in der Entlohnung des im Eigenbesitz befindlichen Bodens, der bei VARD mit dem Erlöskoeffizienten multipliziert wird. Im Hinblick auf die ausgewählten Untersuchungsregionen und auf das Ziel, eine möglichst vergleichbare Vorgehensweise wie im IFCN anzuwenden, wird in dieser Analyse dennoch der Koeffizient „Anteil Milchrinder am Gesamtbestand der Rinder“ beibehalten.

Abschließend wird kurz auf die Datenkontrollen eingegangen, die Grundvoraussetzung aller Berechnungen ist. 
Tabelle A6.3: $\quad$ Schätzung und Aufteilungsschlüssel für die familieneigenen Produktionsfaktoren

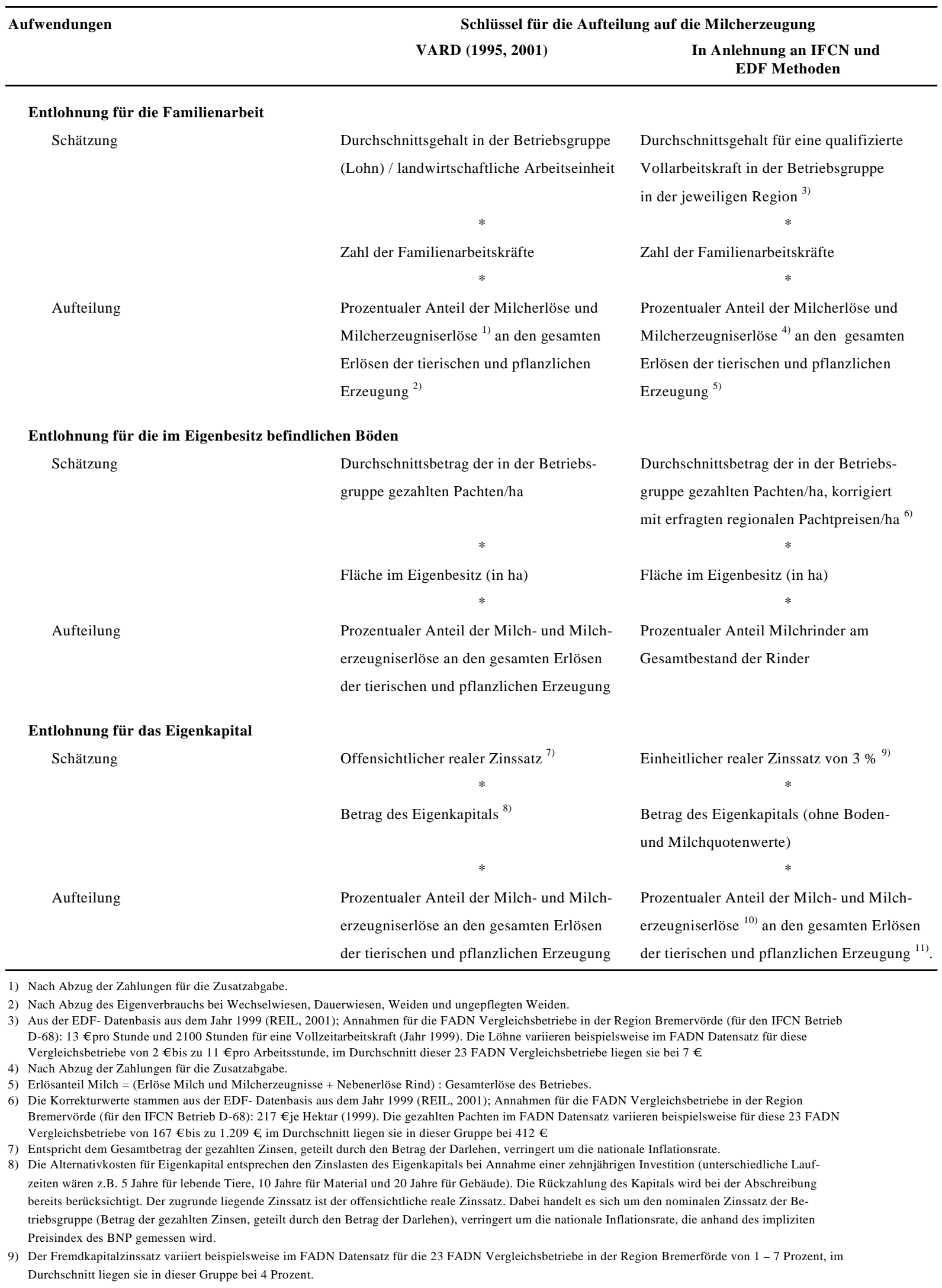




\section{Datenkontrollen}

Alle FADN-Daten unterliegen Plausibilitätstests im Hinblick auf die Einkommensrechnung. Bei einzelnen Angaben kann es dennoch Inkonsistenten geben. Deshalb ist es notwendig, für alle verwendeten FADN-Daten vor der weiteren Analyse intensive Datenkontrollen durchzuführen. Einzelne Schritte dazu sind in Tabelle A6.4 aufgeführt. Beispielhaft soll die Datenprüfung für die Arbeitseinheiten und den Lohnaufwand erläutert werden:

Bei der Datenkontrolle des FADN-Arbeitsaufwandes wird wie folgt vorgegangen:

- Die Arbeitsstunden pro Kuh sollten im Plausibilitätsbereich liegen, der im KTBL (KURATORIUM FÜR TECHNIK UND BAUWESEN IN DER LANDWIRTSCHAFT, VERSCHIEDENE JAHRGÄNGE) dokumentiert ist. Liegen die Arbeitsstunden pro Kuh außerhalb des Plausibilitätsintervalls, wird der Lohn pro Fremdarbeitskraftstunde überprüft. Ist der Lohn plausibel, werden die Familienarbeitskrafteinheiten korrigiert, so dass die Gesamtstundenzahl pro Kuh für den Betrieb und Standort plausibel ist.

- Wenn der Lohn für Fremdarbeitskräfte das Dreifache des jeweiligen EDF ${ }^{\mathbf{0}}$ Vergleichlohns überschreitet, werden die Fremdarbeitskrafteinheiten korrigiert. ${ }^{11}$

- Sind die Fremdarbeitskrafteinheiten angegeben aber kein Lohnaufwand, so werden die Fremdarbeitskrafteinheiten auf 0 gesetzt.

- Ist der Lohnaufwand angegeben aber keine Fremdarbeitskrafteinheiten, werden die Fremdarbeitskrafteinheiten mit Hilfe des EDF-Lohns ermittelt ${ }^{12}$ und korrigiert.

Sind die Stundenzahl pro Kuh und der Lohn pro Fremdarbeitskraftstunde nicht plausibel, werden der Lohnaufwand und/oder die Fremdarbeitskrafteinheiten mit Hilfe des EDFVergleichlohns korrigiert. Stimmt nach erneutem Check die Stundenzahl pro Kuh nicht, fällt der Betrieb aus der Stichprobe heraus.

Folgendes Ablaufdiagramm (Abbildung A6.1) liegt der beschriebenen Datenkontrolle der FADN-Daten im Bereich der Arbeitsaufwendungen zugrunde:

10

Aus der Datenbank der European Dairy Farmers. EDF-AnAlysis (2001), REIL (2001).

11 Das Plausibilitätsintervall variiert je nach Betrieb und Standort. Es werden Korrekturen für maximal $20 \%$ der Betriebe zugelassen.

12

Im FADN-Datensatz variieren die Stunden pro Vollarbeitskraft pro Jahr von 1300 Stunden (in ThrakiMakedonia, Griechenland) bis zu mehr als 3.300 Stunden (Belgien). Für Vergleiche zwischen Ländern und Regionen schien die Verwendung dieser Angaben wenig sinnvoll. Daher wurde in Anlehnung an IFCN- und EDF-Methoden ein Durchschnittswert von 2.100 Stunden für eine Vollarbeitskraft angenommen. Dieser wurde in Abhängigkeit vom Betrieb und Region angepasst. 
Abbildung A6.1: $\quad$ Ablaufdiagramm zur Kontrolle der FADN-Arbeitsaufwendungen

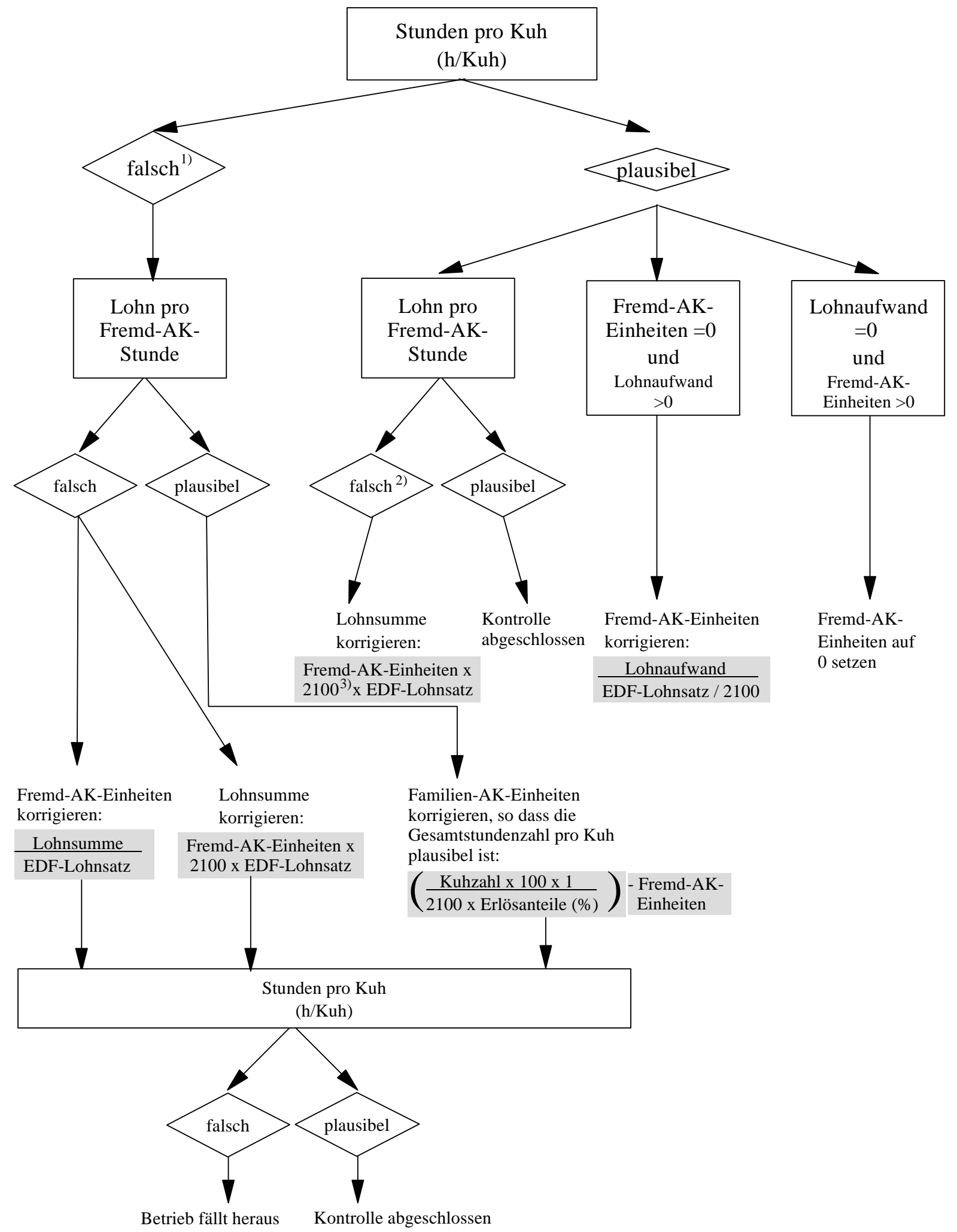

1) Die Werte gelten hier als falsch, wenn sie die dreifachen EDF-Arbeitskraftstunden übersteigen.

2) Die Werte gelten hier als falsch, wenn sie den dreifachen EDF-Lohnsatz übersteigen.

3) Richtwert für die Stunden pro Vollarbeitskraft; der Wert wurde in Abhängigkeit vom Betrieb und Standort angepasst.

Quelle: Eigene Darstellung (2004). 
Tabelle A6.4: Weitere Datenkontrollen

„Erlöse und Subventionen“

- Der Wert für Erlöse und Subventionen für Milch, Rindfleisch und die gesamte Produktion müssen positiv sein;

- Milch- und Rindfleischerlöse inklusiv Subventionen dürfen nicht höher als die Gesamterlöse inklusiv Subventionen sein;

Betriebe, die diese Bedingungen nicht erfüllen, werden aus der Stichprobe eliminiert.

\section{„Pachtaufwendungen Acker- und Grünland“}

Pachtflächen sind im FADN nicht nach Acker- und Dauergrünlandflächen differenziert. Daher wird ein Näherungswert für Acker- und Grünlandpachtflächen hergeleitet:

- Pachtumfang Grünland:

(Gesamtfläche Grünland/Gesamte Landwirtschaftliche Nutzfläche) * Gesamtes Pachtland

- Kontrolle:

Grünland gesamt - Pacht Grünland = Inputvariable Eigentumsfläche Grünland

Grünland gesamt $=$ Eigenfläche + Pachtfläche Grünland

- Mit der Pacht- bzw. Eigenfläche von Ackerland wird genauso verfahren.

Kontrolle: Gesamte Pachtfläche (V27) > = Pacht Grünland + Pacht Ackerland

Tabelle A6.5: $\quad$ Strukturelle Entwicklung von Milchviehbetrieben in Deutschland

\begin{tabular}{|c|c|c|c|c|c|c|c|c|c|c|c|c|}
\hline & 1990 & 1991 & 1992 & 1993 & 1994 & 1995 & 1996 & 1997 & 1998 & 1999 & 2000 & 2001 \\
\hline Anzahl Milchviehbetriebe & 291 & 275 & 256 & 236 & 223 & 210 & 198 & 186 & 169 & 153 & 142 & 132 \\
\hline Änderung zum Vorjahr & & $-6 \%$ & $-7 \%$ & $-8 \%$ & $-6 \%$ & $-6 \%$ & $-6 \%$ & $-6 \%$ & $-9 \%$ & $-10 \%$ & $-7 \%$ & $-7 \%$ \\
\hline Ø Herdengröße (Anzal & 17 & 17 & 20 & 23 & 24 & 25 & 26 & 28 & 29 & 31 & 33 & 35 \\
\hline$\%$ Betriebe $>50$ Kühe & $3 \%$ & $3 \%$ & $4 \%$ & $6 \%$ & $7 \%$ & $8 \%$ & $10 \%$ & $11 \%$ & $12 \%$ & $14 \%$ & $16 \%$ & $18 \%$ \\
\hline \% Betriebe > 100 Kühe & $0,1 \%$ & $0,1 \%$ & $0,3 \%$ & $1,2 \%$ & $1,4 \%$ & $1,6 \%$ & $1,8 \%$ & $2,1 \%$ & $2,3 \%$ & $2,5 \%$ & $2,9 \%$ & $3,4 \%$ \\
\hline
\end{tabular}

Quelle: Hemme et. al (2003): IFCN Dairy Report 2003; auf Basis von FAO (Produktionsjahrbuch), Eurostat, ZMP, IMF und nationalen Statistiken (Statistisches Bundesamt). 
Tabelle A6.6: $\quad$ Strukturelle Entwicklung von Milchviehbetrieben in Schweden

\begin{tabular}{lcrrrrrrrrrrr}
\hline & $\mathbf{1 9 9 0}$ & $\mathbf{1 9 9 1}$ & $\mathbf{1 9 9 2}$ & $\mathbf{1 9 9 3}$ & $\mathbf{1 9 9 4}$ & $\mathbf{1 9 9 5}$ & $\mathbf{1 9 9 6}$ & $\mathbf{1 9 9 7}$ & $\mathbf{1 9 9 8}$ & $\mathbf{1 9 9 9}$ & $\mathbf{2 0 0 0}$ & $\mathbf{2 0 0 1}$ \\
\hline $\begin{array}{l}\text { Anzahl Milchviehbetriebe in 1.000 } \\
\text { Änderung zum Vorjahr }\end{array}$ & 24,8 & 21,5 & 19,8 & 18,9 & 18,0 & 17,2 & 15,9 & 15,0 & 14,2 & 13,2 & 12,2 & 11,3 \\
& & $-13 \%$ & $-8 \%$ & $-5 \%$ & $-5 \%$ & $-4 \%$ & $-8 \%$ & $-6 \%$ & $-5 \%$ & $-7 \%$ & $-8 \%$ & $-7 \%$ \\
& & & & & & & & & & & & \\
Ø Herdengröße (Anzahl Kühe) & 22 & 23 & 25 & 26 & 27 & 28 & 29 & 31 & 32 & 34 & 35 & 37 \\
\% Betriebe > 50 Kühe & & & & $11 \%$ & $10 \%$ & $10 \%$ & $12 \%$ & $13 \%$ & $15 \%$ & $17 \%$ & $21 \%$ & $22 \%$ \\
\% Betriebe > 100 Kühe & & & & $0,0 \%$ & $0,0 \%$ & $1,2 \%$ & $1,6 \%$ & $2,0 \%$ & $2,1 \%$ & $2,3 \%$ & $4,1 \%$ & $4,4 \%$ \\
\hline
\end{tabular}

Quelle: Hemme et. al (2003): IFCN Dairy Report 2003; auf Basis von FAO (Produktionsjahrbuch), ZMP, IMF, nationalen Statistiken (Svensk Mjölk invãgningsstatistik) und Schätzungen von P. Nordgren.

Tabelle A6.7: $\quad$ Strukturelle Entwicklung von Milchviehbetrieben in Spanien

\begin{tabular}{|c|c|c|c|c|c|c|c|c|c|c|}
\hline & 1990 & 1991 & 1992 & 1993 & 1994 & 1995 & 1996 & 1997 & 1998 & 1999 \\
\hline Anzahl Milchviehbetriebe in 1.000 & 208 & 185 & 167 & 148 & 133 & 118 & 112 & 106 & 88 & 69 \\
\hline Änderung zum Vorjahr & & $-11 \%$ & $-10 \%$ & $-11 \%$ & $-10 \%$ & $-11 \%$ & $-5 \%$ & $-5 \%$ & $-17 \%$ & $-21 \%$ \\
\hline Ø Herdengröße (Anzahl Kühe) & 8 & 8 & 9 & 9 & 10 & 11 & 11 & 12 & 14 & 17 \\
\hline$\%$ Betriebe $>50$ Kühe & $1,2 \%$ & $1,6 \%$ & $1,8 \%$ & $2,0 \%$ & $2,1 \%$ & $2,2 \%$ & $2,5 \%$ & $2,7 \%$ & $3,9 \%$ & $5,8 \%$ \\
\hline$\%$ Betriebe $>100$ Kühe & $0,3 \%$ & $0,5 \%$ & $0,6 \%$ & $0,7 \%$ & $0,6 \%$ & $0,5 \%$ & $0,5 \%$ & $0,6 \%$ & $0,9 \%$ & $1,4 \%$ \\
\hline
\end{tabular}

Quelle: Hemme et. al (2003): IFCN Dairy Report 2003; auf Basis von FAO (Produktionsjahrbuch), Eurostat, ZMP, IMF, nationalen Statistiken (Statistisches Bundesamt) und Schätzungen von E. Reyes. 


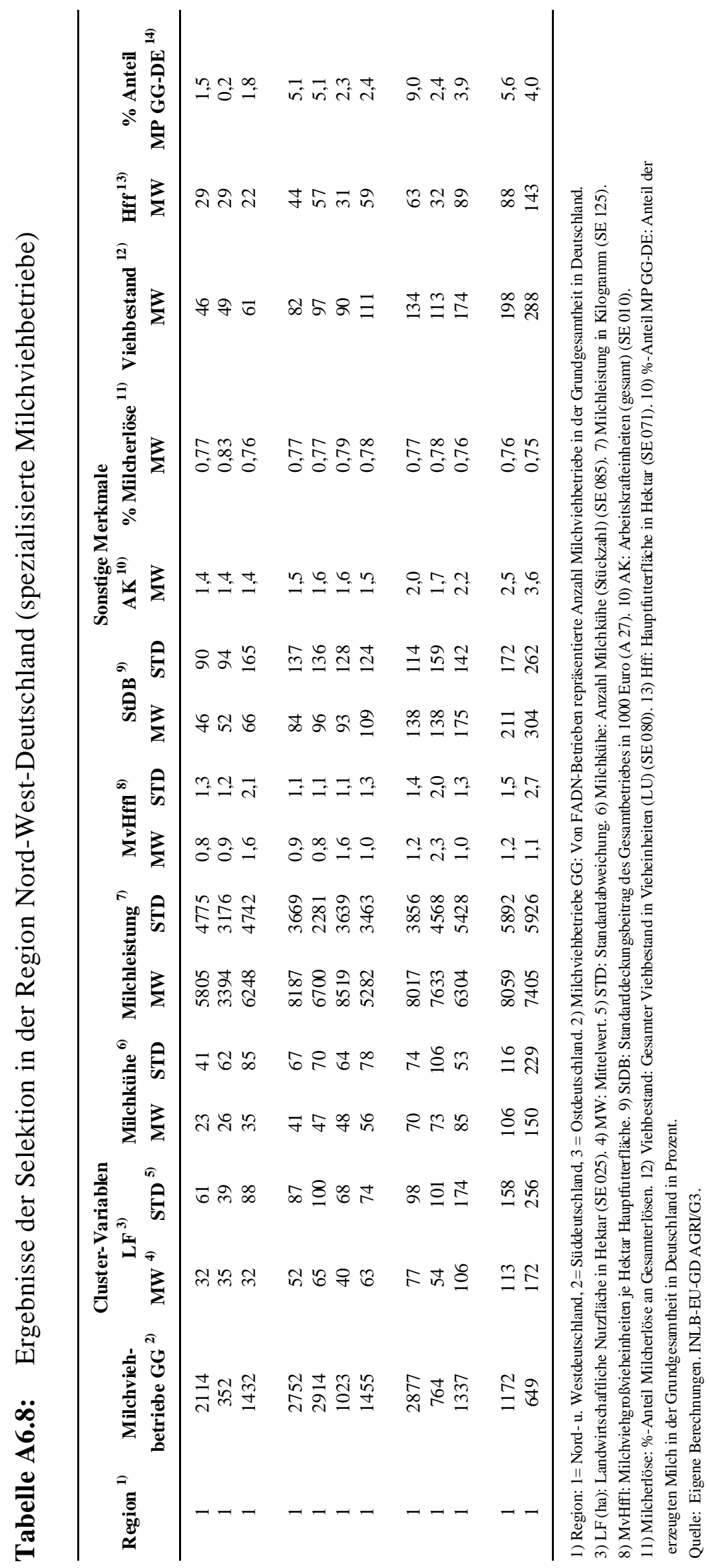




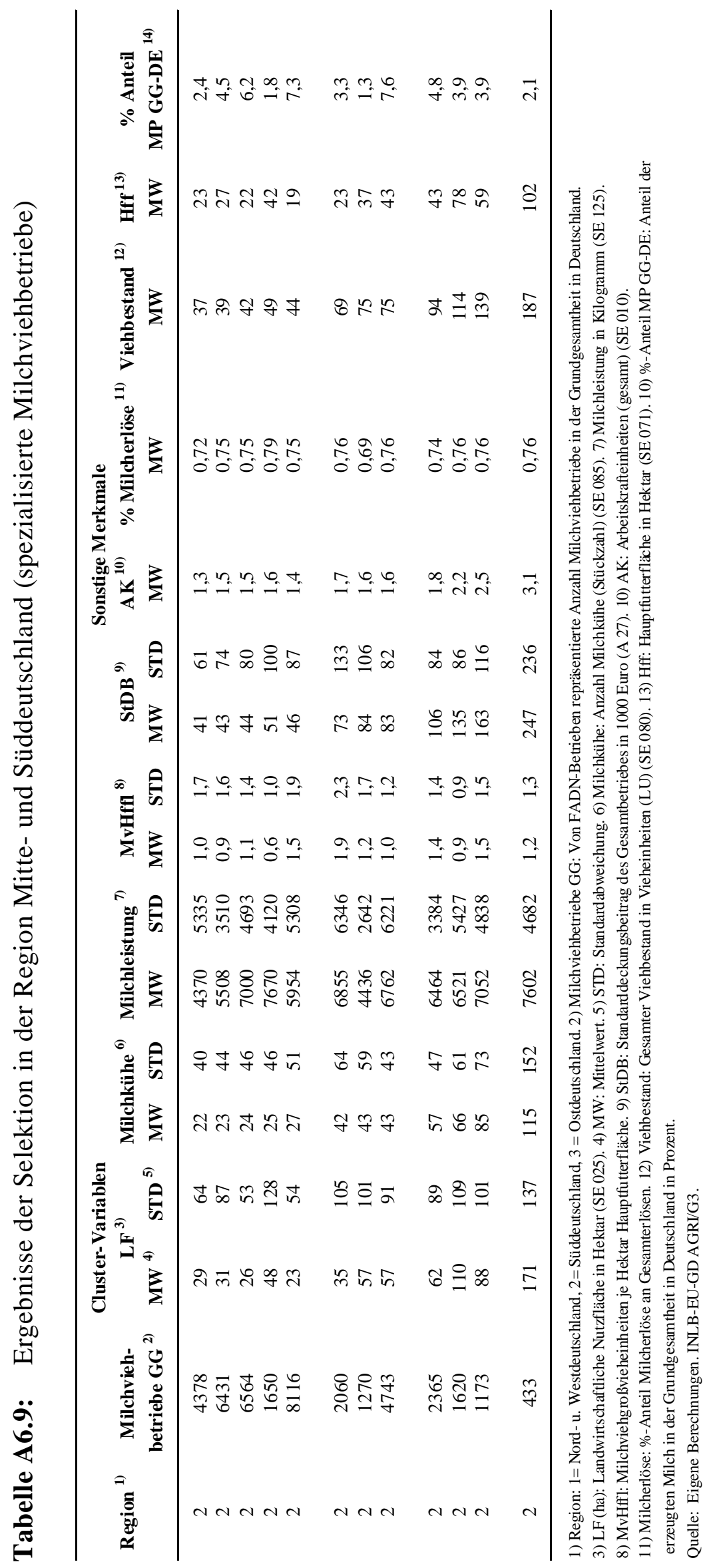




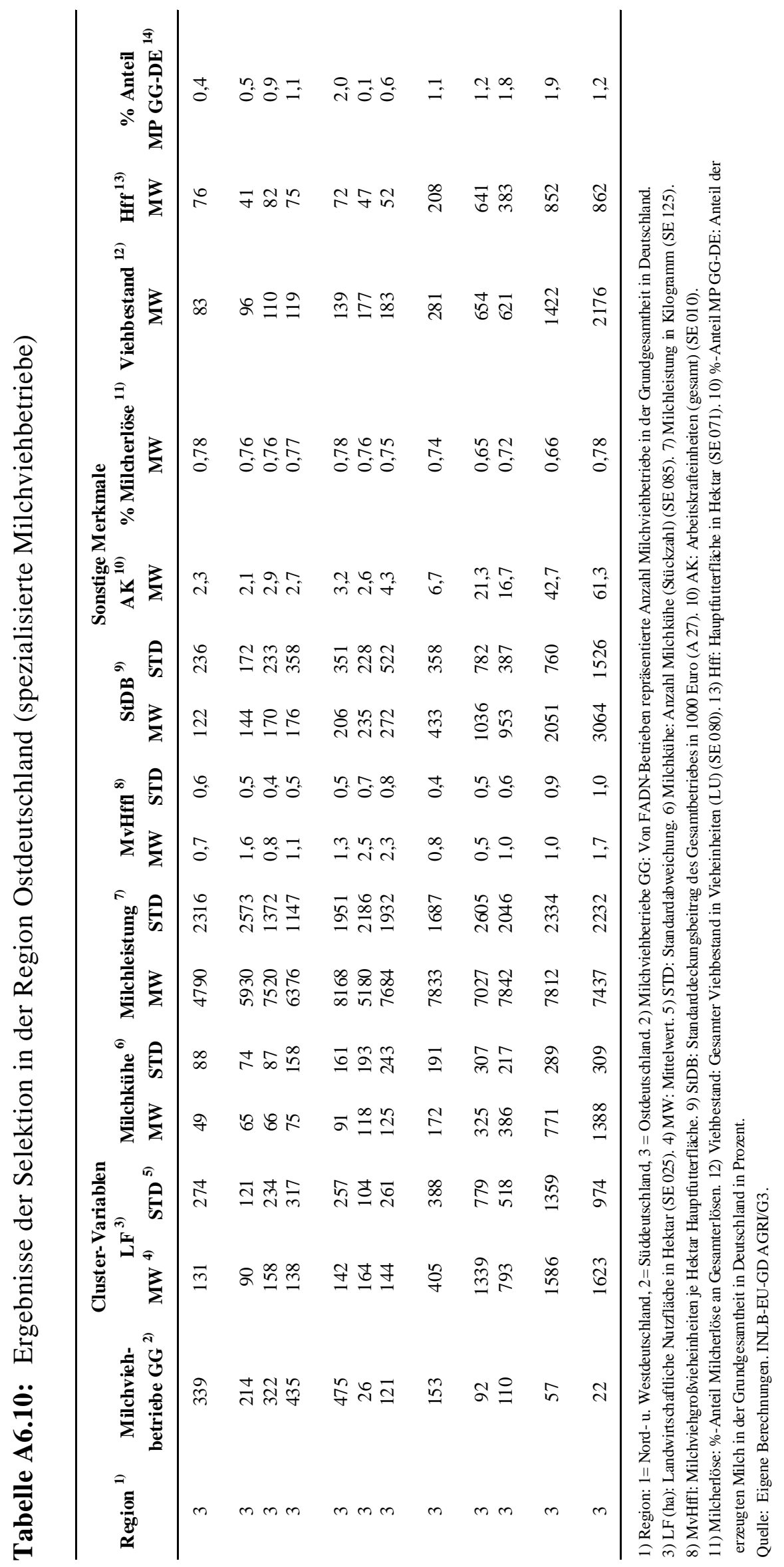




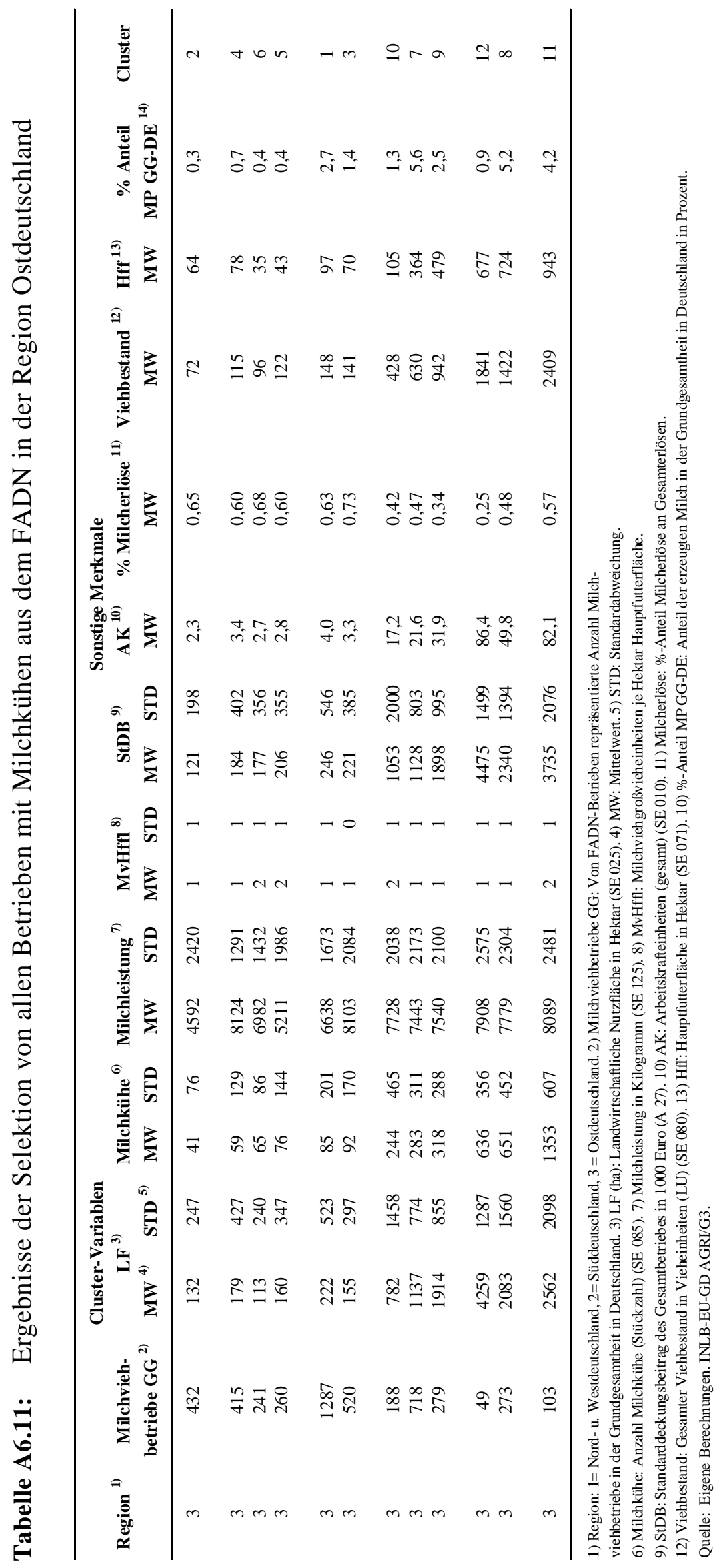




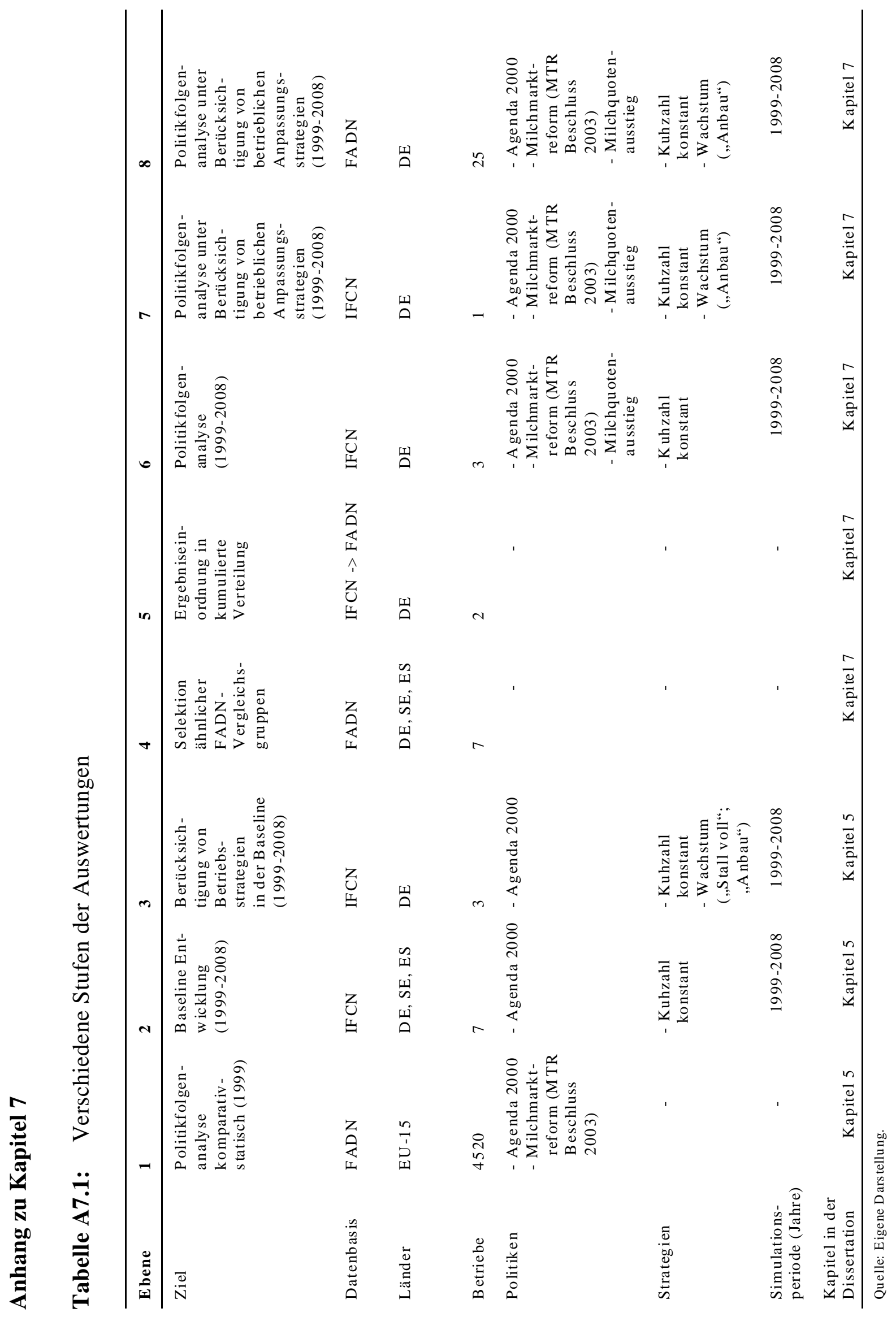




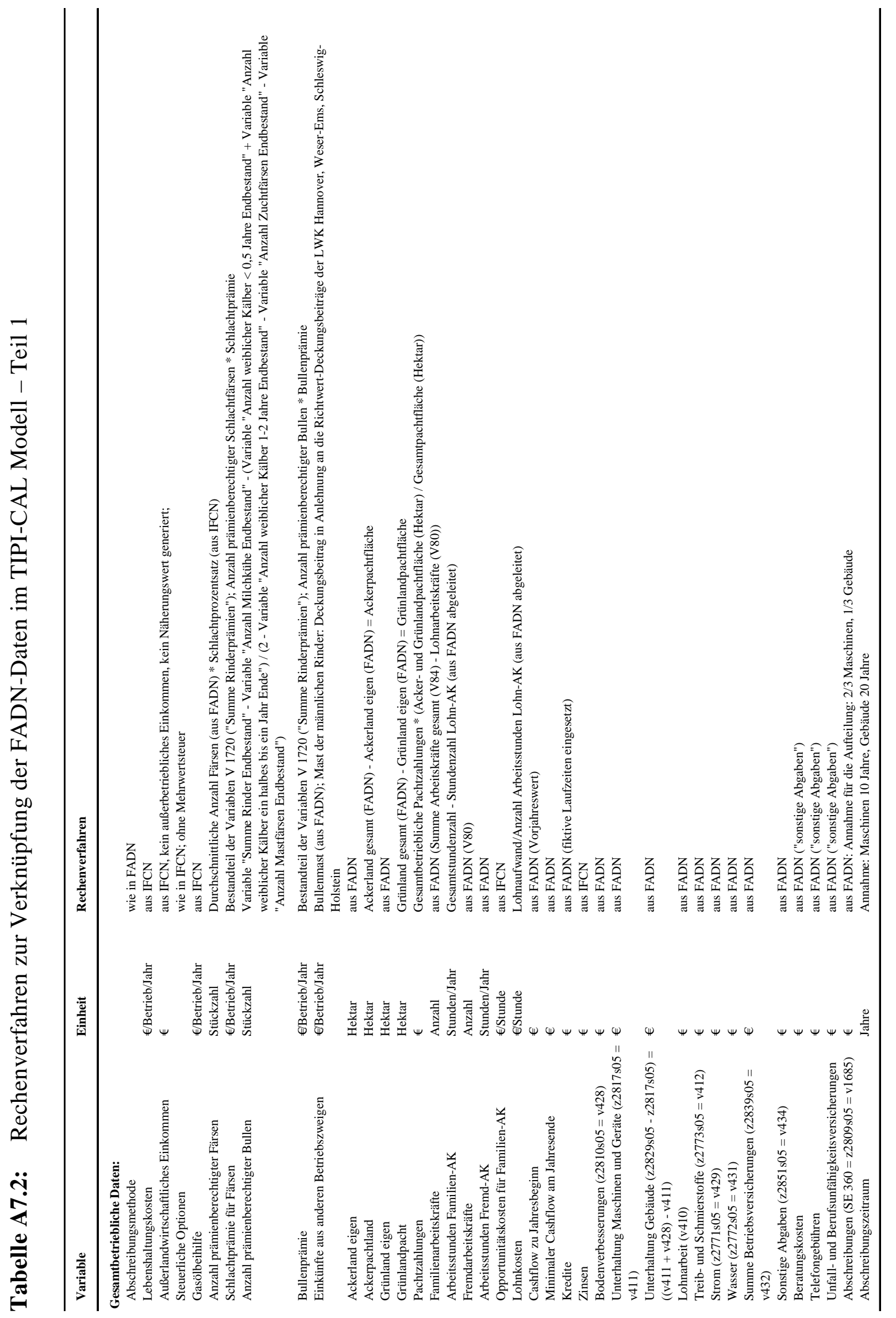




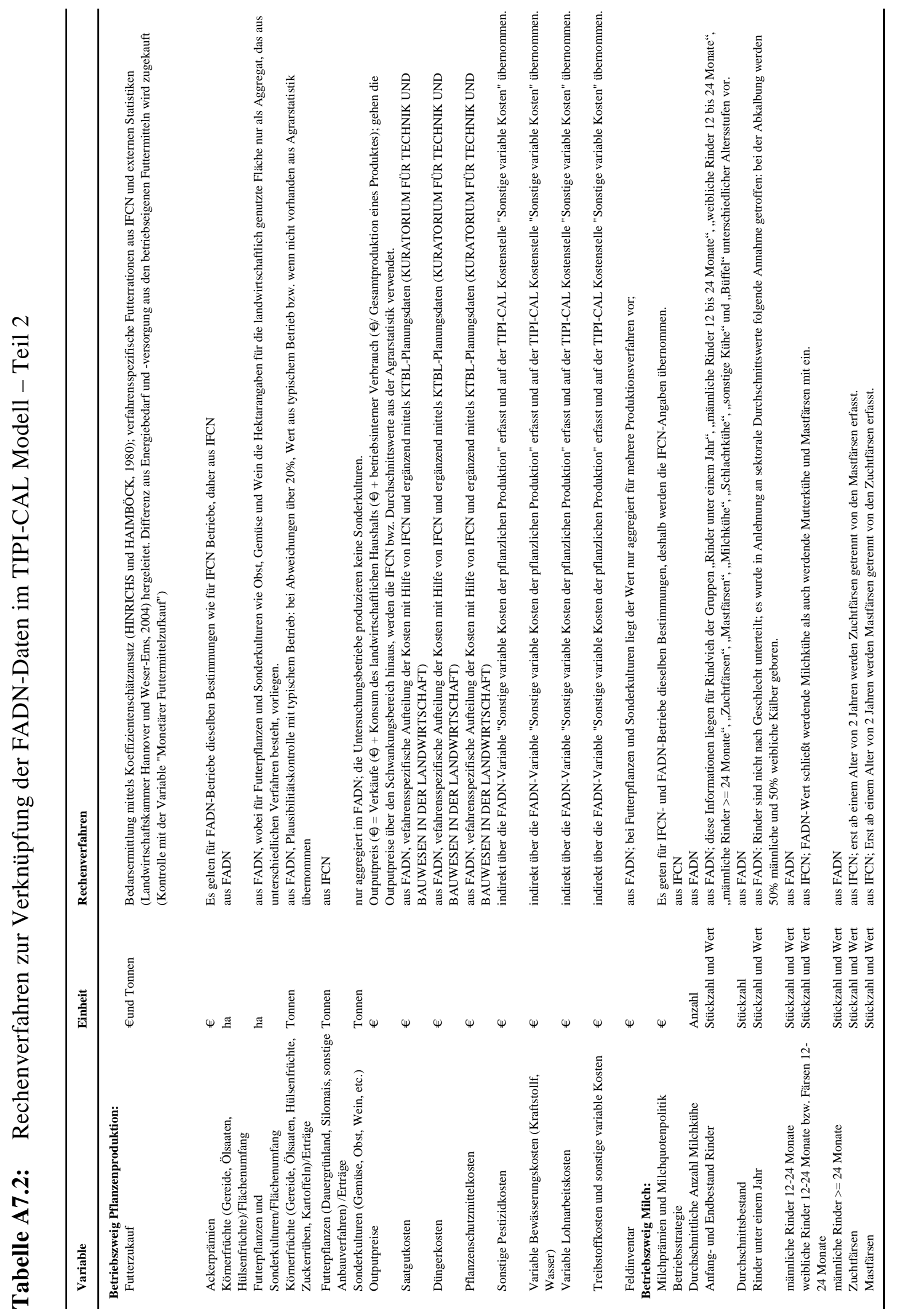




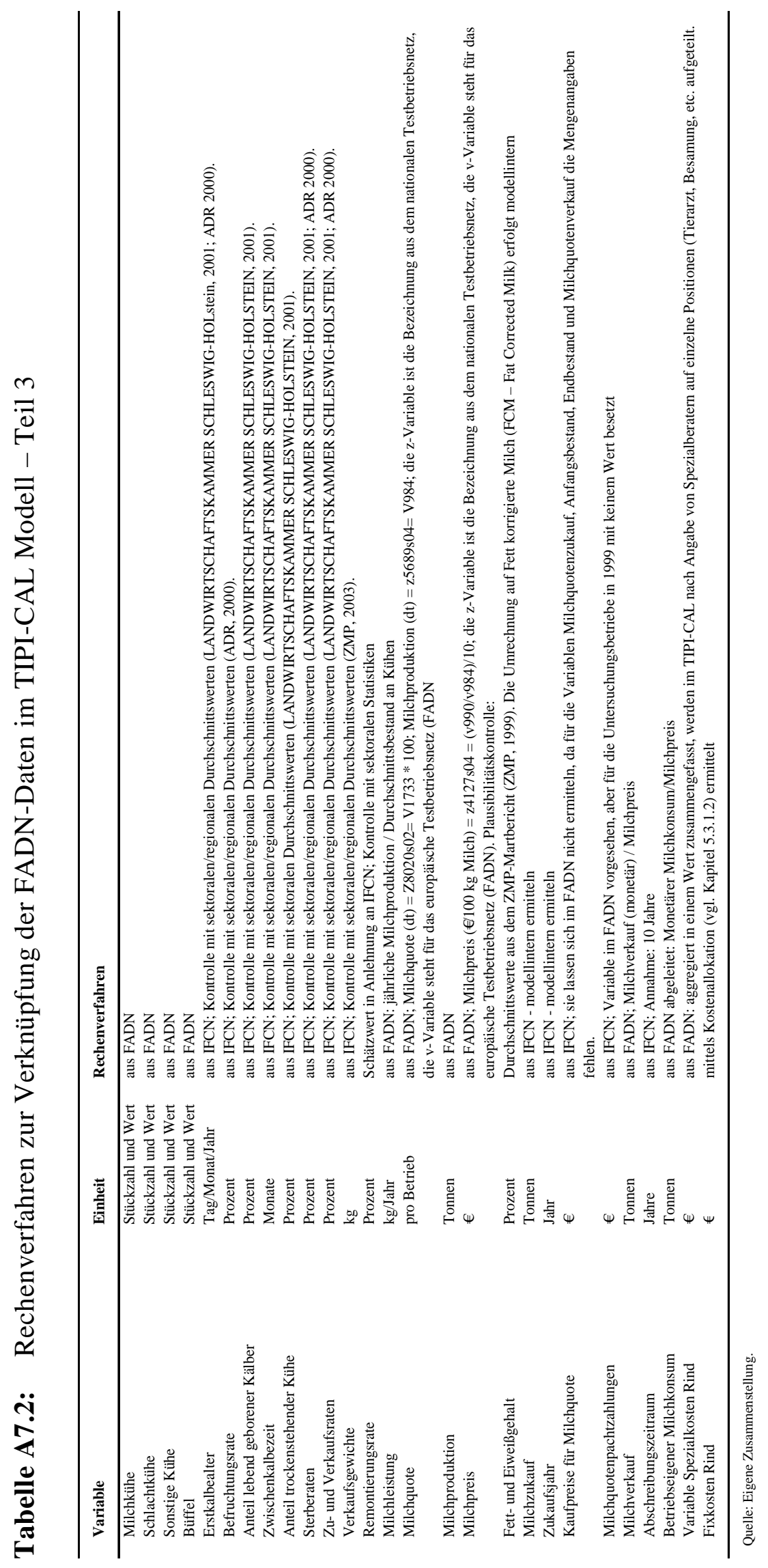

